\title{
The Halicylindramides, Farnesoid X Receptor
}

\section{Antagonizing Depsipeptides from a Petrosia sp.}

\section{Marine Sponge Collected in Korea}

Dongyup Hahn, ${ }^{\dagger}$ Hiyoung Kim,${ }^{\ddagger}$ Inho Yang,,${ }^{\dagger}$ Jungwook Chin,${ }^{\dagger}$ Hoosang Hwang, ${ }^{\dagger}$ Dong Hwan Won, ${ }^{\ddagger}$ Byoungchan Lee, ${ }^{\star}$ Sang-Jip Nam, ${ }^{\S}$ Merrick Ekins, ${ }^{\perp}$ Hyukjae Choi, ${ }^{*, \mid}$ and Heonjoong Kang*,

${ }^{\dagger}$ New Drug Development Center, Daegu-Gyeongbuk Medical Innovation Foundation, Daegu, 41061, Korea

${ }^{\ddagger}$ School of Earth and Environmental Sciences, Seoul National University, NS-80, Seoul, 08826, Republic of Korea

${ }^{\S}$ Department of Chemistry and Nano Science, Global Top 5 Program, Ewha Womans University, Seoul, 03760, Republic of Korea

\footnotetext{
${ }^{\perp}$ Queensland Museum, P.O. Box 3300, South Brisbane, Queensland, 4101, Australia "College of Pharmacy, Yeungnam University, Gyeongsan, 38541, Republic of Korea
} 


\section{Table of Contents}

Table S1. NMR spectroscopic data for halicylindramide F (1) in DMSO-d6 at $600 \mathrm{MHz}\left({ }^{1} \mathrm{H}\right)$ and $150 \mathrm{MHz}\left({ }^{13} \mathrm{C}\right)$.

Table S2. Absolute configuration of halicylindramide F (1) by advanced Marfey's method and Edman degradation .7

Table S3. NMR spectroscopic data for halicylindramide G (2) in DMSO- $d_{6}$ at $600 \mathrm{MHz}\left({ }^{1} \mathrm{H}\right)$ and $150 \mathrm{MHz}\left({ }^{13} \mathrm{C}\right)$.

Table S4. NMR spectroscopic data for halicylindramide H (3) ) in DMSO-d 6 at $600 \mathrm{MHz}\left({ }^{1} \mathrm{H}\right)$ and $150 \mathrm{MHz}\left({ }^{13} \mathrm{C}\right)$....

Table S5. Absolute configuration of halicylindramides G (2) and H (3) by advanced Marfey's method and Edman degradation

S6. HR-ESI-Tof-MS spectrum of halicylindramide F (1) ........................... 13

S7. ${ }^{1} \mathrm{H}$ NMR (600 MHz) spectrum of halicylindramide F (1) in DMSO-d6 ................. 14

S8. ${ }^{13} \mathrm{C}$ NMR (150 MHz) spectrum of halicylindramide F (1) in DMSO-d $d_{6} \ldots \ldots \ldots \ldots \ldots . . . .15$

S9. HMQC (600 MHz) spectrum of halicylindramide F (1) in DMSO-d $\ldots \ldots \ldots \ldots \ldots \ldots$

S10. COSY (600 MHz) spectrum of halicylindramide F (1) in DMSO-d $\ldots \ldots \ldots \ldots \ldots \ldots 17$

S11. Expanded COSY (600 MHz) spectrum of halicylindramide F (1) in DMSO-d $d_{6} \ldots \ldots .18$

S12. Expanded COSY (600 MHz) spectrum of halicylindramide F (1) in DMSO- $d_{6} \ldots \ldots . .19$

S13. TOCSY (600 MHz) spectrum of halicylindramide F (1) in DMSO-d $\ldots \ldots \ldots \ldots \ldots . .20$

S14. Expanded TOCSY (600 MHz) spectrum of halicylindramide F (1) in DMSO-d $d_{6} \ldots . . .21$

S15. Expanded TOCSY (600 MHz) spectrum of halicylindramide F (1) in DMSO-d $\ldots . . . .22$

S16. Expanded TOCSY (600 MHz) spectrum of halicylindramide F (1) in DMSO-d $\ldots . . .23$

S17. NOESY (600 MHz) spectrum of halicylindramide F (1) in DMSO-d $6 \ldots \ldots \ldots \ldots . . .24$

S18. Expanded NOESY (600 MHz) spectrum of halicylindramide F (1) in DMSO-d $\ldots . . .25$

S19. Expanded NOESY (600 MHz) spectrum of halicylindramide F (1) in DMSO-d $\ldots . . .26$

S20. Expanded NOESY (600 MHz) spectrum of halicylindramide F (1) in DMSO-d $\ldots . . .27$

S21. HMBC (600 MHz) spectrum of halicylindramide F (1) in DMSO-d $\ldots \ldots \ldots \ldots \ldots \ldots . . .28$

S22. Expanded HMBC (600 MHz) spectrum of halicylindramide F (1) in DMSO-d $\ldots . . . .29$

S23. Expanded HMBC (600 MHz) spectrum of halicylindramide F (1) in DMSO-d $\ldots . . . .30$

S24. Expanded HMBC (600 MHz) spectrum of halicylindramide F (1) in DMSO-d $d_{6} \ldots . . .31$

S25. Expanded HMBC (600 MHz) spectrum of halicylindramide F (1) in DMSO-d $\ldots . . . .32$ 
S26. Expanded HMBC (600 MHz) spectrum of halicylindramide F (1) in DMSO- $d_{6} \ldots . . . .33$

S27. Expanded HMBC (600 MHz) spectrum of halicylindramide F (1) in DMSO- $d_{6} \ldots . . .34$

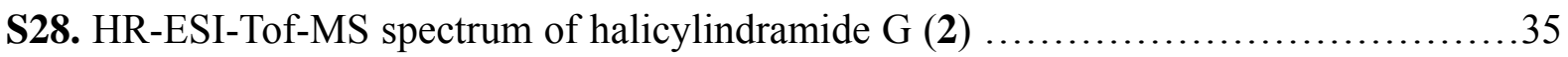

S29. ${ }^{1} \mathrm{H}$ NMR (600 MHz) spectrum of halicylindramide G (2) in DMSO-d6 ................. 36

S30. ${ }^{13} \mathrm{C}$ NMR (150 MHz) spectrum of halicylindramide G (2) in DMSO-d6 ................37

S31. HSQC (600 MHz) spectrum of halicylindramide G (2) in DMSO-d $6 \ldots \ldots \ldots \ldots \ldots . . . . . .38$

S32. COSY (600 MHz) spectrum of halicylindramide G (2) in DMSO-d $6 \ldots \ldots \ldots \ldots \ldots . . . . . .39$

S33. Expanded COSY (600 MHz) spectrum of halicylindramide G (2) in DMSO-d $\ldots . . . .40$

S34. Expanded COSY (600 MHz) spectrum of halicylindramide G (2) in DMSO-d $\ldots . . . .41$

S35. TOCSY (600 MHz) spectrum of halicylindramide G (2) in DMSO-d $d_{6} \ldots \ldots \ldots \ldots . . . . .42$

S36. Expanded TOCSY (600 MHz) spectrum of halicylindramide G (2) in DMSO-d6 ......43

S37. Expanded TOCSY (600 MHz) spectrum of halicylindramide G (2) in DMSO-d $\ldots . . .44$

S38. Expanded TOCSY (600 MHz) spectrum of halicylindramide G (2) in DMSO-d $\ldots . . .45$

S39. NOESY (600 MHz) spectrum of halicylindramide G (2) in DMSO- $d_{6} \ldots \ldots \ldots \ldots \ldots . . \ldots 6$

S40. Expanded NOESY (600 MHz) spectrum of halicylindramide G (2) in DMSO-d $\ldots . . .47$

S41. Expanded NOESY (600 MHz) spectrum of halicylindramide G (2) in DMSO-d $\ldots . . .48$

S42. Expanded NOESY (600 MHz) spectrum of halicylindramide G (2) in DMSO-d $\ldots . . .49$

S43. Expanded NOESY (600 MHz) spectrum of halicylindramide G (2) in DMSO-d $\ldots . . .50$

S44. HMBC (600 MHz) spectrum of halicylindramide G (2) in DMSO-d $\ldots \ldots \ldots \ldots \ldots . . .51$

S45. Expanded HMBC (600 MHz) spectrum of halicylindramide G (2) in DMSO-d $d_{6} \ldots . .52$

S46. Expanded HMBC (600 MHz) spectrum of halicylindramide G (2) in DMSO-d $\ldots . . .53$

S47. Expanded HMBC (600 MHz) spectrum of halicylindramide G (2) in DMSO-d $\ldots . . .54$

S48. Expanded HMBC (600 MHz) spectrum of halicylindramide G (2) in DMSO- $d_{6} \ldots . .55$

S49. Expanded HMBC (600 MHz) spectrum of halicylindramide G (2) in DMSO-d $d_{6} \ldots . .56$

S50. Expanded HMBC (600 MHz) spectrum of halicylindramide G (2) in DMSO-d $\ldots . . .57$

S51. Expanded HMBC (600 MHz) spectrum of halicylindramide G (2) in DMSO-d $d_{6} \ldots . .58$

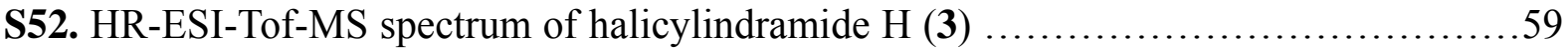

S53. ${ }^{1} \mathrm{H}$ NMR (600 MHz) spectrum of halicylindramide H (3) in DMSO-d6..............60

S54. ${ }^{13} \mathrm{C}$ NMR (150 MHz) spectrum of halicylindramide H (3) in DMSO-d6...............61

S55. HSQC (600 MHz) spectrum of halicylindramide H (3) in DMSO-d $\ldots \ldots \ldots \ldots \ldots \ldots . . . .62$

S56. COSY (600 MHz) spectrum of halicylindramide H (3) in DMSO- $d_{6} \ldots \ldots \ldots \ldots \ldots . . . .63$ 
S57. Expanded COSY (600 MHz) spectrum of halicylindramide H (3) in DMSO-d $d_{6} \ldots . . . .64$

S58. Expanded COSY (600 MHz) spectrum of halicylindramide H (3) in DMSO-d $\ldots . . . .65$

S59. Expanded COSY (600 MHz) spectrum of halicylindramide H (3) in DMSO-d $\ldots . . . . .66$

S60. Expanded COSY (600 MHz) spectrum of halicylindramide H (3) in DMSO-d6 .......67

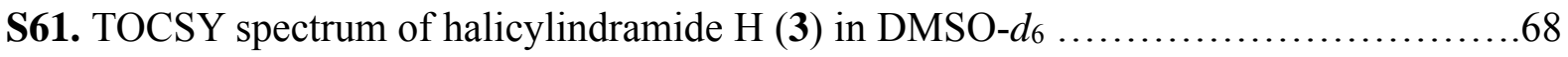

S62. Expanded TOCSY (600 MHz) spectrum of halicylindramide H (3) in DMSO-d $\ldots . . .69$

S63. Expanded TOCSY (600 MHz) spectrum of halicylindramide H (3) in DMSO-d $\ldots . . .70$

S64. Expanded TOCSY (600 MHz) spectrum of halicylindramide H (3) in DMSO-d $\ldots . . .71$

S65. NOESY (600 MHz) spectrum of halicylindramide H (3) in DMSO- $d_{6} \ldots \ldots \ldots \ldots \ldots . . . . .72$

S66. Expanded NOESY (600 MHz) spectrum of halicylindramide H (3) in DMSO-d $\ldots . . .73$

S67. Expanded NOESY (600 MHz) spectrum of halicylindramide H (3) in DMSO-d6 .....74

S68. Expanded NOESY (600 MHz) spectrum of halicylindramide H (3) in DMSO-d $d_{6} \ldots . .75$

S69. Expanded NOESY (600 MHz) spectrum of halicylindramide H (3) in DMSO-d6 .....76

S70. Expanded NOESY (600 MHz) spectrum of halicylindramide H (3) in DMSO-d $\ldots . . .77$

S71. HMBC (600 MHz) spectrum of halicylindramide H (3) in DMSO-d $6 \ldots \ldots \ldots \ldots . . . . .78$

S72. Expanded HMBC (600 MHz) spectrum of halicylindramide H (3) in DMSO-d $\ldots . . .79$

S73. Expanded HMBC (600 MHz) spectrum of halicylindramide H (3) in DMSO- $d_{6} \ldots . .80$

S74. Expanded HMBC (600 MHz) spectrum of halicylindramide H (3) in DMSO-d $d_{6} \ldots . .81$

S75. Expanded HMBC (600 MHz) spectrum of halicylindramide H (3) in DMSO-d $d_{6} \ldots . .82$

S76. Expanded HMBC (600 MHz) spectrum of halicylindramide H (3) in DMSO-d $d_{6} \ldots . .83$

S77. Expanded HMBC (600 MHz) spectrum of halicylindramide H (3) in DMSO-d $\ldots . . .84$

S78. Expanded HMBC (600 MHz) spectrum of halicylindramide H (3) in DMSO-d $d_{6} \ldots . .85$

S79. Expanded HMBC (600 MHz) spectrum of halicylindramide H (3) in DMSO- $d_{6} \ldots . .86$

S80. Expanded HMBC (600 MHz) spectrum of halicylindramide H (3) in DMSO- $d_{6} \ldots . .87$

S81. Expanded HMBC (600 MHz) spectrum of halicylindramide H (3) in DMSO-d $d_{6} \ldots . .88$

S82. Expanded HMBC (600 MHz) spectrum of halicylindramide H (3) in DMSO- $d_{6} \ldots . .89$

S83. Indirect binding of $\mathbf{1}, \mathbf{4}$ and 5 to the LBD of hFXR on BIAcore experiments ..........90

S84. Underwater photograph of the animal specimen and Photograph of the animal specimen and taxonomic description

S85. Specific rotation values, ECD absorption values, ${ }^{1} \mathrm{H}$ NMR $(600 \mathrm{MHz})$ data and LR-ESIMS data of synthetic Dioia. 
Table S1. NMR spectroscopic data for halicylindramide F (1) in DMSO-d 6 at $600 \mathrm{MHz}\left({ }^{1} \mathrm{H}\right)$ and $150 \mathrm{MHz}\left({ }^{13} \mathrm{C}\right)$

\begin{tabular}{|c|c|c|c|c|c|c|}
\hline position & $\delta_{\mathrm{C}}$, mult. $^{\mathrm{a}}$ & $\delta_{\mathrm{H}},(\mathrm{J}$ in $\mathrm{Hz})$ & COSY & TOCSY & NOESY & HMBC \\
\hline $\mathrm{CHO}$ & $160.5, \mathrm{C}$ & $7.89, \mathrm{~s}$ & Ala-NH & Ala-NH, Ala $\alpha$, Ala $\beta$ & Ala-NH & Ala $\alpha$ \\
\hline Ala $\alpha$ & $46.4, \mathrm{CH}$ & $4.33, \mathrm{q}(7.3)$ & $\mathrm{NH}, \beta$ & $\mathrm{NH}, \beta, \mathrm{CHO}$ & Phe1-NH & $\beta, \mathrm{C}=\mathrm{O}, \mathrm{CHO}$ \\
\hline$\beta$ & $19.4, \mathrm{CH}_{3}$ & $0.89, \mathrm{~d}(7.2)$ & $\alpha$ & $\mathrm{NH}, \alpha$ & & $\alpha, \mathrm{C}=\mathrm{O}$ \\
\hline $\mathrm{NH}$ & & 8.14, brd $(7.8)$ & $\alpha, \mathrm{CHO}$ & $\mathrm{CHO}, \alpha, \beta$ & $\mathrm{CHO}$ & $\mathrm{CHO}$ \\
\hline $\mathrm{CO}$ & $171.4, \mathrm{C}$ & & & & & \\
\hline Phe1 $\alpha$ & $51.7, \mathrm{CH}$ & $4.72, \mathrm{~m}$ & $\mathrm{NH}, \beta 2$ & $\mathrm{NH}, \beta 2$ & & \\
\hline$\beta 1$ & $34.7, \mathrm{CH}_{2}$ & $2.74, \mathrm{~d}(13.8)$ & $\beta 2$ & $\beta 2$ & Pro $\delta$ & $\mathrm{C}=\mathrm{O}$ \\
\hline$\beta 2$ & & $3.01, \mathrm{~m}$ & $\alpha, \beta 1$ & $\mathrm{NH}, \alpha, \beta 1$ & & $1,2,6, \mathrm{C}=\mathrm{O}$ \\
\hline $\mathrm{C} 1$ & 137.7, C & & & & & \\
\hline $\mathrm{C} 2 / \mathrm{C} 6$ & $129.3, \mathrm{CH}$ & $7.25, \mathrm{~m}$ & 3,5 & $3,4,5$ & & $\beta, 1,3,4,5$ \\
\hline $\mathrm{C} 3 / \mathrm{C} 5$ & $128.0, \mathrm{CH}$ & $7.22, \mathrm{~m}$ & $2,4,6$ & $2,4,6$ & & $2,4,6$ \\
\hline $\mathrm{C} 4$ & 126.7, $\mathrm{CH}$ & $7.16, \mathrm{~m}$ & 3,5 & $2,3,5,6$ & & $2,3,5,6$ \\
\hline $\mathrm{NH}$ & & 8.45, brd $(8.4)$ & $\alpha$ & $\alpha, \beta 1, \beta 2$ & Ala $\alpha$ & \\
\hline $\mathrm{CO}$ & $169.4, \mathrm{C}$ & & & & & \\
\hline Pro $\alpha$ & $59.8, \mathrm{CH}$ & $4.46, \mathrm{~m}$ & $\beta 1, \beta 2$ & $\beta 1, \beta 2, \gamma$ & Val-NH & \\
\hline$\beta 1$ & $29.8, \mathrm{CH}_{2}$ & $1.88, \mathrm{~m}$ & $\alpha, \gamma$ & $\alpha, \beta 2, \gamma, \delta$ & & \\
\hline$\beta 2$ & & $2.07 \mathrm{~m}$ & $\alpha, \gamma$ & $\alpha, \beta 1, \gamma, \delta$ & & $\mathrm{C}=\mathrm{O}$ \\
\hline$\gamma$ & $24.4, \mathrm{CH}_{2}$ & $1.89, \mathrm{~m}$ & $\beta 1, \beta 2, \delta$ & $\alpha, \beta 1, \beta 2, \delta$ & & \\
\hline$\delta$ & $47.0, \mathrm{CH}_{2}$ & $3.67, \mathrm{~m}$ & $\gamma$ & $\beta, \gamma$ & Phe1 $\beta 1$ & \\
\hline $\mathrm{CO}$ & $171.1, \mathrm{C}$ & & & & & \\
\hline Val $\alpha$ & $57.2, \mathrm{CH}$ & $4.38, \mathrm{~m}^{\mathrm{b}}$ & $\mathrm{NH}, \beta$ & $\mathrm{NH}, \beta, \gamma, \gamma^{\prime}$ & $t$-Leu-NH & $\beta, \gamma, \gamma^{\prime}, \mathrm{C}=\mathrm{O}$ \\
\hline$\beta$ & $31.2, \mathrm{CH}$ & $2.07, \mathrm{~m}$ & $\alpha, \gamma, \gamma^{\prime}$ & $\mathrm{NH}, \alpha, \gamma, \gamma^{\prime}$ & & $\alpha, \gamma, \gamma^{\prime}$ \\
\hline$\gamma$ & $17.3, \mathrm{CH}_{3}$ & $0.78, \mathrm{~d}(6.7)$ & $\beta$ & $\alpha, \beta, \gamma^{\prime}$ & & $\alpha, \beta, \gamma^{\prime}$ \\
\hline$\gamma^{\prime}$ & $18.7, \mathrm{CH}_{3}$ & $0.83, \mathrm{~d}(7.2)$ & $\beta$ & $\alpha, \beta, \gamma$ & & $\alpha, \beta, \gamma$ \\
\hline $\mathrm{NH}$ & & $7.81, \mathrm{~m}$ & $\alpha$ & $\alpha, \beta$ & Pro $\alpha$ & Pro $\mathrm{C}=\mathrm{O}$ \\
\hline $\mathrm{CO}$ & $171.0, \mathrm{C}$ & & & & & \\
\hline$t$-Leu $\alpha$ & $60.4, \mathrm{CH}$ & $4.18, \mathrm{~d}(8.7)$ & $\mathrm{NH}$ & $\mathrm{NH}$ & Trp-NH & $\beta, \gamma, C=O$ \\
\hline$\beta$ & $33.7, \mathrm{C}$ & & & & & \\
\hline$\gamma$ & $26.5, \mathrm{CH}_{3}$ & $0.70, \mathrm{~s}$ & & & & $\alpha, \beta$ \\
\hline $\mathrm{NH}$ & & $7.76, \mathrm{~m}$ & $\alpha$ & $\alpha$ & Val $\alpha$ & \\
\hline $\mathrm{CO}$ & $169.9, \mathrm{C}$ & & & & & \\
\hline $\operatorname{Trp} \alpha$ & $54.0, \mathrm{CH}$ & $4.57, \mathrm{~m}$ & $\mathrm{NH}, \beta 1, \beta 2$ & $\beta 1, \beta 2, \mathrm{NH}$ & Arg-NH & $\beta, 3, C=O$ \\
\hline$\beta 1$ & $26.8, \mathrm{CH}_{2}$ & $3.16, \mathrm{~m}$ & $\alpha, \beta 2$ & $\alpha, \beta 2, \mathrm{NH}$ & & 2,3 \\
\hline$\beta 2$ & & $2.96, \mathrm{~m}$ & $\alpha, \beta 1$ & $\alpha, \beta 1, \mathrm{NH}$ & & 2,3 \\
\hline $\mathrm{C} 2$ & $124.1, \mathrm{CH}$ & $7.15, \mathrm{~s}$ & $1-\mathrm{NH}$ & $1-\mathrm{NH}$ & & $3,3 a, 7 a$ \\
\hline $\mathrm{C} 3$ & 109.8, C & & & & & \\
\hline $\mathrm{C} 3 \mathrm{a}$ & $136.6, \mathrm{C}$ & & & & & \\
\hline $\mathrm{C} 7 \mathrm{a}$ & $119.5, \mathrm{C}$ & & & & & \\
\hline $\mathrm{C} 4$ & $118.1, \mathrm{CH}$ & $7.60, \mathrm{~d}(7.9)$ & 5 & $5,6,7$ & & $2,3,3 a, 6$ \\
\hline $\mathrm{C} 5$ & $120.8, \mathrm{CH}$ & 6.96 , dd $(7.5,7.1)$ & 4,6 & $4,6,7$ & & $3 a, 6,7$ \\
\hline C6 & $111.2, \mathrm{CH}$ & 7.03 , dd $(7.4,7.6)$ & 5,7 & $4,5,7$ & & $5,7 \mathrm{a}$ \\
\hline C7 & $127.2, \mathrm{CH}$ & $7.31, \mathrm{~d}(8.0)$ & 6 & $4,5,6$ & & $5,7 \mathrm{a}$ \\
\hline $1-\mathrm{NH}$ & & 10.6, brs & 2 & 2 & & \\
\hline $\mathrm{NH}$ & & 8.29, brd (6.5) & $\alpha$ & $\alpha$ & $t$-Leu $\alpha$ & $t$-Leu $\mathrm{C}=\mathrm{O}$ \\
\hline $\mathrm{CO}$ & $170.0, \mathrm{C}$ & & & & & \\
\hline $\operatorname{Arg} \alpha$ & $51.5, \mathrm{CH}$ & $4.39, \mathrm{~m}$ & $\beta, \mathrm{NH}$ & $\beta, \gamma, \mathrm{NH}$ & $\mathrm{Cys}\left(\mathrm{SO}_{3} \mathrm{H}\right)-\mathrm{NH}$ & $\beta, \gamma, \mathrm{C}=\mathrm{O}$ \\
\hline$\beta$ & $29.5, \mathrm{CH}_{2}$ & $1.61, \mathrm{~m}$ & $\alpha, \gamma$ & $\alpha, \gamma, \delta$ & & \\
\hline$\gamma$ & 23.6, $\mathrm{CH}_{2}$ & $1.28, \mathrm{~m}$ & $\beta, \delta$ & $\alpha, \beta, \delta$ & & \\
\hline$\delta$ & $39.7, \mathrm{CH}_{2}$ & $2.94, \mathrm{~m}$ & $\gamma$ & $\beta, \gamma$ & & \\
\hline guanidine & 156.7, C & & & & & \\
\hline $\mathrm{NH}$ & & 7.99, brs & $\alpha$ & $\alpha$ & $\operatorname{Trp} \alpha$ & $\operatorname{Trp} \mathrm{C}=\mathrm{O}$ \\
\hline $\mathrm{CO}$ & $171.4, \mathrm{C}$ & & & & & \\
\hline $\mathrm{Cys}\left(\mathrm{SO}_{3} \mathrm{H}\right) \alpha$ & $50.9, \mathrm{CH}$ & $4.60, \mathrm{~m}$ & $\beta, \mathrm{NH}$ & $\beta, \mathrm{NH}$ & Thr1-NH & $\beta, C=O$ \\
\hline$\beta$ & $52.4, \mathrm{CH}_{2}$ & $2.93, \mathrm{~m}$ & $\alpha$ & $\alpha, \mathrm{NH}$ & & \\
\hline $\mathrm{NH}$ & & 8.35, brd $(6.5)$ & $\alpha$ & $\alpha, \beta$ & $\operatorname{Arg} \alpha$ & $\operatorname{Arg} \mathrm{C}=\mathrm{O}$ \\
\hline $\mathrm{CO}$ & $170.8, \mathrm{C}$ & & & & & \\
\hline Thr1 $\alpha$ & $51.8, \mathrm{CH}$ & $4.83, \mathrm{~d}(8.5)$ & NH & $\beta, \mathrm{NH}$ & NMeGln-NMe & $\mathrm{C}=\mathrm{O}$ \\
\hline$\beta$ & $69.5, \mathrm{CH}$ & $5.14, \mathrm{~d}(6.7)$ & $\gamma$ & $\alpha, \gamma$ & & $\mathrm{C}=\mathrm{O}, \mathrm{Sar} \mathrm{C}=\mathrm{O}$ \\
\hline$\gamma$ & $17.3, \mathrm{CH}_{3}$ & $1.16, \mathrm{~d}(6.5)$ & $\beta$ & $\alpha$ & & $\alpha, \beta$ \\
\hline $\mathrm{NH}$ & & $7.98, \mathrm{~m}$ & $\alpha$ & $\alpha, \beta$ & $\mathrm{Cys}\left(\mathrm{SO}_{3} \mathrm{H}\right) \alpha$ & $\mathrm{Cys}\left(\mathrm{SO}_{3} \mathrm{H}\right) \mathrm{C}=\mathrm{O}$ \\
\hline $\mathrm{CO}$ & $167.9, \mathrm{C}$ & & & & & \\
\hline $\mathrm{NMeG} \ln \alpha$ & $54.9, \mathrm{CH}$ & $4.97, \mathrm{~m}$ & $\beta 1$ & $\beta, \gamma$ & Phe-NH & \\
\hline$\beta 1$ & $24.7, \mathrm{CH}_{2}$ & $1.70, \mathrm{~m}$ & $\alpha, \gamma$ & $\alpha, \gamma$ & & $\mathrm{CONH}_{2}$ \\
\hline$\beta 2$ & & $1.89, \mathrm{~m}^{\mathrm{b}}$ & & $\alpha, \beta 1, \gamma$ & & \\
\hline$\gamma$ & $31.6, \mathrm{CH}_{2}$ & $1.87, \mathrm{~m}^{\mathrm{b}}$ & $\beta 1$ & $\alpha, \beta 1, \beta 2$ & & $\alpha, \beta, \mathrm{CONH}_{2}$ \\
\hline $\mathrm{NMe}$ & $30.6, \mathrm{CH}_{3}$ & $2.93, \mathrm{~s}$ & & & Thr1 $\alpha$ & $\alpha$, Thr1 $\mathrm{C}=\mathrm{O}$ \\
\hline $\mathrm{CONH}_{2}$ & 173.6, C & 6.68 , brs & & & & $\gamma, \mathrm{CONH}_{2}$ \\
\hline 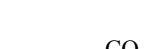 & & 7.17 , brs & & & & $\gamma, \mathrm{CONH}_{2}$ \\
\hline
\end{tabular}




\begin{tabular}{|c|c|c|c|c|c|c|}
\hline Phe $2 \alpha$ & $54.2, \mathrm{CH}$ & $4.67, \mathrm{~d}(7.2)$ & $\mathrm{NH}$ & $\mathrm{NH}, \beta$ & Thr2-NH & $\beta, 1, C=O$ \\
\hline$\beta$ & $38.0, \mathrm{CH}_{2}$ & $3.00, \mathrm{~m}$ & $\alpha$ & $\mathrm{NH}, \alpha$ & & $\alpha, 1,2,6, \mathrm{C}=\mathrm{O}$ \\
\hline $\mathrm{C} 1$ & $137.3, \mathrm{C}$ & & & & & \\
\hline $\mathrm{C} 2 / \mathrm{C} 6$ & $129.4, \mathrm{CH}$ & $7.22, \mathrm{~m}$ & 3,5 & $3,4,5$ & & $\beta, 1,3,4,5$ \\
\hline $\mathrm{C} 3 / \mathrm{C} 5$ & $128.0, \mathrm{CH}$ & $7.14, \mathrm{~m}$ & $2,4,6$ & $2,4,6$ & & $1,2,4,6$ \\
\hline $\mathrm{C} 4$ & 126.3, CH & $7.12, \mathrm{~m}$ & 3,5 & $2,3,5,6$ & & $2,3,5,6$ \\
\hline $\mathrm{NH}$ & & 7.55, brd $(6.6)$ & $\alpha$ & $\alpha, \beta$ & $\mathrm{NMeG} \ln \alpha$ & $\mathrm{NMeG} \ln \mathrm{C}=\mathrm{O}$ \\
\hline $\mathrm{CO}$ & $171.1, \mathrm{C}$ & & & & & \\
\hline Thr $2 \alpha$ & $60.9, \mathrm{CH}$ & $3.85, \mathrm{~m}$ & $\mathrm{NH}, \beta$ & $\mathrm{NH}, \beta, \gamma, \mathrm{OH}$ & Asn-NH & $\mathrm{C}=\mathrm{O}$ \\
\hline$\beta$ & $65.6, \mathrm{CH}$ & $3.96, \mathrm{~m}$ & $\alpha, \gamma, \mathrm{OH}$ & $\gamma, \mathrm{NH}, \mathrm{OH}$ & & $\alpha$ \\
\hline$\gamma$ & $20.2, \mathrm{CH}_{3}$ & $0.89, \mathrm{~d}(4.8)$ & $\beta$ & $\alpha, \beta, \mathrm{OH}$ & & $\alpha, \beta$ \\
\hline $\mathrm{OH}$ & & $4.87, \mathrm{~d}(5.1)$ & $\beta$ & $\alpha, \beta, \gamma$ & & \\
\hline $\mathrm{NH}$ & & 7.92, brd $(7.9)$ & $\alpha$ & $\alpha, \beta$ & Phe $\alpha$ & \\
\hline $\mathrm{CO}$ & $169.8, \mathrm{C}$ & & & & & \\
\hline Asn $\alpha$ & $45.9, \mathrm{CH}$ & $4.98, \mathrm{~m}$ & $\mathrm{NH}, \beta 1, \beta 2$ & $\mathrm{NH}, \alpha, \beta 1, \beta 2$ & Sar-NMe & $\beta, \mathrm{CONH}_{2}$ \\
\hline$\beta 1$ & $36.9, \mathrm{CH}_{2}$ & $2.08, \mathrm{~m}$ & $\alpha$ & $\mathrm{NH}, \alpha, \beta 1, \beta 2$ & & $\alpha, \mathrm{C}=\mathrm{O}, \mathrm{CONH}_{2}$ \\
\hline$\beta 2$ & & $2.64, \mathrm{~m}$ & $\alpha$ & $\mathrm{NH}, \alpha, \beta 1, \beta 2$ & & \\
\hline $\mathrm{CONH}_{2}$ & $171.2, \mathrm{C}$ & 6.78, brs & $\mathrm{CONH}_{2}$ & $\mathrm{CONH}_{2}$ & & $\mathrm{C}=\mathrm{O}, \mathrm{Thr} 2 \mathrm{C}=\mathrm{O}$ \\
\hline & & 7.32, brs & $\mathrm{CONH}_{2}$ & $\mathrm{CONH}_{2}$ & & $\beta, \mathrm{CONH}_{2}$ \\
\hline $\mathrm{NH}$ & & 7.50, brd $(9.6)$ & $\alpha$ & $\mathrm{NH}, \alpha, \beta 1, \beta 2$ & $\operatorname{Thr} 2 \alpha$ & $\beta, \mathrm{CONH}_{2}$ \\
\hline $\mathrm{CO}$ & $172.1, \mathrm{C}$ & & & & & \\
\hline \multirow[t]{2}{*}{$\operatorname{Sar} \alpha$} & $48.6, \mathrm{CH}_{2}$ & $3.46, \mathrm{~d}(15.7)$ & $\alpha 2$ & $\alpha 2$ & & $\mathrm{C}=\mathrm{O}$ \\
\hline & & $4.40, \mathrm{~m}$ & $\alpha 1$ & $\alpha 1$ & & $\mathrm{C}=\mathrm{O}$ \\
\hline $\mathrm{NMe}$ & $35.6, \mathrm{CH}_{3}$ & $2.73, \mathrm{~s}$ & & & Asn $\alpha$ & $\alpha$, Asn $\mathrm{C}=\mathrm{O}$ \\
\hline $\mathrm{CO}$ & $167.6, \mathrm{C}$ & & & & & \\
\hline
\end{tabular}

${ }^{a}$ Derived from the combination of ${ }^{13} \mathrm{C}$ NMR, HSQC and HMBC data. ${ }^{b}$ Singals overlapped. 
Table S2. Absolute configuration of halicylindramide F (1) by advanced Marfey's method and Edman degradation

\begin{tabular}{|c|c|c|c|c|c|}
\hline \multirow[t]{2}{*}{ Parent peptide } & \multirow{2}{*}{$\begin{array}{l}\text { Constitutive } \\
\text { amino acids }\end{array}$} & \multirow[t]{2}{*}{$m / z$} & \multicolumn{2}{|c|}{ Retention time } & \multirow{2}{*}{$\begin{array}{c}\text { Absolute } \\
\text { configuration of } \\
\text { amino acids }\end{array}$} \\
\hline & & & AA-L-DLA & AA-D-DLA & \\
\hline \multirow[t]{11}{*}{1} & $\mathrm{Ala}^{a}$ & $495-496$ & 39.08 & 33.99 & $\mathrm{D}$ \\
\hline & Pro $^{a}$ & $521.5-522.5$ & 33.89 & 37.28 & $\mathrm{~L}$ \\
\hline & $\mathrm{Val}^{a}$ & $523-524$ & 46.90 & 38.97 & $\mathrm{D}$ \\
\hline & $t-\mathrm{Leu}^{a}$ & $537-538$ & 41.33 & 50.39 & $\mathrm{~L}$ \\
\hline & $\operatorname{Trp}^{a}$ & 610.5-611.5 & 46.25 & 41.42 & $\mathrm{D}$ \\
\hline & $\operatorname{Arg}^{b, c}$ & 694-695 & 26.28 & 21.32 & $\mathrm{~L}$ \\
\hline & $\mathrm{Cys}\left(\mathrm{SO}_{3} \mathrm{H}\right)^{a, c}$ & $575-576$ & 11.30 & 12.74 & $\mathrm{D}$ \\
\hline & Thr1, Thr2 ${ }^{a}$ & $525-526$ & 27.73 & 33.90 & $\mathrm{~L}$ \\
\hline & $\mathrm{NMeG} \ln ^{a}$ & $567-568$ & 28.84 & 29.69 & $\mathrm{~L}$ \\
\hline & Phe1, Phe ${ }^{d}$ & $457.5-458.8$ & $49.32 / 43.57$ & $49.32 / 43.50$ & D \& L \\
\hline & $\operatorname{Asn}^{d}$ & $524.5-525.0$ & 36.99 & 36.99 & \\
\hline \multirow[t]{2}{*}{ Standard amino acids } & L-allo-Thr & $525-526$ & 28.82 & 31.27 & \\
\hline & $\mathrm{L}-\mathrm{Asn}^{e}$ & $384.5-385.5$ & $\begin{array}{c}47.80 \\
\text { L-AA-L-DAA }\end{array}$ & $\begin{array}{c}56.20 \\
\text { D-AA-L-DAA }\end{array}$ & \\
\hline 1 & $\mathrm{Asn}^{e}$ & $384.5-385.5$ & 47.76 & & $\mathrm{~L}$ \\
\hline Dodecapeptide of $\mathbf{1}^{f}$ & Phe $2^{d}$ & $457.5-458.8$ & 48.78 & 42.99 & $\mathrm{D}$ \\
\hline
\end{tabular}

a The ions of advanced Marfey's reaction products were detected as form of $\left[\mathrm{M}+\mathrm{CF}_{3} \mathrm{COO}\right]^{-}$under negative ion detection mode.

${ }^{b}$ The ions of advanced Marfey's reaction products were detected as form of $\left[\mathrm{M}+2 \mathrm{CF}_{3} \mathrm{COOH}-\mathrm{H}\right]^{-}$under negative ion detection mode.

${ }^{c}$ The L-AA-D-DLA or D-AA-L-DLA products derived from the amino acids with highly polar residues such as Arg and $\mathrm{Cys}\left(\mathrm{SO}_{3} \mathrm{H}\right)$ elute faster than L-AA-L-DLA or D-AA-D-DLA products (Fuji et al., Anal. Chem. 1997, 69, 5146-5151).

${ }^{\mathrm{d}}$ The ions of advanced Marfey's reaction products were detected as form of [M-H] under negative ion detection mode.

e The absolute configuration of Asn was determined by Marfey's method (with based on the comparison of retention time of natural Asn-L-DAA to those of two authentic standards (L-Asn-L-DAA 47.80/ D-Asn-L-DAA $56.20 \mathrm{~min})$.

${ }^{\mathrm{f}}$ Dodecapeptide of $\mathbf{1}$ was prepared by deformylation followed by two cycles of Edman degradation. 
Table S3. NMR spectroscopic data for halicylindramide G (2) in DMSO-d 6 at $600 \mathrm{MHz}\left({ }^{1} \mathrm{H}\right)$ and $150 \mathrm{MHz}\left({ }^{13} \mathrm{C}\right)$

\begin{tabular}{|c|c|c|c|c|c|c|}
\hline position & $\delta_{\mathrm{c}}$, mult. $^{\mathrm{a}}$ & $\delta_{\mathrm{H}},(\mathrm{J}$ in $\mathrm{Hz})$ & COSY & TOCSY & NOESY & HMBC ( $\mathrm{H}$ to $\mathrm{C}$ ) \\
\hline $\mathrm{NCHO}$ & $160.5, \mathrm{C}$ & $7.88, \mathrm{~s}$ & Ala-NH & Ala-NH, Ala $\alpha$, Ala $\beta$ & Ala-NH & \\
\hline Ala $\alpha$ & $46.4, \mathrm{CH}$ & $4.31, \mathrm{q}(7.3)$ & $\mathrm{NH}, \beta$ & $\mathrm{NH}, \beta$, formyl & $\beta$, formyl, Br-Phe-NH & $\beta$, formyl $\mathrm{C}=\mathrm{O}$ \\
\hline$\beta$ & $18.6, \mathrm{CH}_{3}$ & $0.93, d(7.0)$ & $\alpha$ & $\mathrm{NH}, \alpha$ & $\alpha$ & $\alpha, \mathrm{C}=\mathrm{O}$ \\
\hline $\mathrm{NH}$ & & 8.18, brs & $\alpha$, formyl & formyl, $\alpha, \beta$ & formyl & \\
\hline $\mathrm{CO}$ & $171.4, \mathrm{C}$ & & & & & \\
\hline BrPhe $\alpha$ & $51.9, \mathrm{CH}$ & $4.71, \mathrm{~m}^{\mathrm{b}}$ & $\mathrm{NH}, \beta 1, \beta 2$ & $\mathrm{NH}, \beta 1, \beta 2$ & Pro $\delta$ & \\
\hline$\beta 1$ & $36.2, \mathrm{CH}_{2}$ & $2.69-2.72 \mathrm{~m}$ & $\alpha, \beta 2$ & $\mathrm{NH}, \alpha, \beta 2$ & $\alpha$ & $1,2,6$ \\
\hline$\beta 2$ & & $3.02, \mathrm{~m}^{\mathrm{b}}$ & $\alpha, \beta 1$ & $\mathrm{NH}, \alpha, \beta 1$ & $\alpha$ & $1,2,6$ \\
\hline $\mathrm{C} 1$ & $137.1, \mathrm{C}$ & & & & & \\
\hline $\mathrm{C} 2 / \mathrm{C} 6$ & $131.8, \mathrm{CH}$ & $7.26, \mathrm{~m}^{\mathrm{b}}$ & 3,5 & 3,5 & 3,5 & $\beta, 1,3,4,6$ \\
\hline $\mathrm{C} 3 / \mathrm{C} 5$ & $130.8, \mathrm{CH}$ & $7.42, \mathrm{~m}$ & 2,6 & 2,6 & 2,6 & 1,4 \\
\hline $\mathrm{C} 4$ & $119.5, \mathrm{C}$ & & & & & \\
\hline $\mathrm{NH}$ & & 8.44, brd $(8.3)$ & $\alpha$ & $\alpha, \beta$ & Ala $\alpha$ & $\mathrm{C}=\mathrm{O}$ \\
\hline $\mathrm{CO}$ & $171.0, \mathrm{C}$ & & & & & \\
\hline Pro $\alpha$ & $59.5, \mathrm{CH}$ & $4.46, \mathrm{~m}$ & $\beta 1, \beta 2$ & $\beta 1, \beta 2, \gamma, \delta$ & Val-NH & \\
\hline$\beta 1$ & $29.5, \mathrm{CH}_{2}$ & $1.89, \mathrm{~m}$ & $\alpha, \gamma$ & $\alpha, \beta 2, \gamma, \delta$ & & \\
\hline$\beta 2$ & & $2.10, \mathrm{~m}$ & $\alpha, \gamma$ & $\alpha, \beta 1, \gamma, \delta$ & & $\mathrm{C}=\mathrm{O}$ \\
\hline$\gamma$ & 24.6, $\mathrm{CH}_{2}$ & $1.90, \mathrm{~m}$ & $\beta 1, \beta 2, \delta$ & $\alpha, \beta 1, \beta 2, \delta$ & & \\
\hline$\delta$ & $46.9, \mathrm{CH}_{2}$ & $3.70-3.62 \mathrm{~m}$ & $\gamma$ & $\alpha, \beta, \gamma$ & BrPhe $\alpha$ & \\
\hline $\mathrm{CO}$ & $169.8, \mathrm{C}$ & & & & & \\
\hline Val $\alpha$ & $57.2, \mathrm{CH}$ & $4.38, \mathrm{~m}^{\mathrm{b}}$ & $\mathrm{NH}, \beta$ & $\mathrm{NH}$ & t-Leu-NH & $\mathrm{C}=\mathrm{O}$ \\
\hline$\beta$ & $30.7, \mathrm{CH}$ & $2.08, \mathrm{~m}$ & $\alpha, \gamma, \gamma^{\prime}$ & $\alpha, \gamma, \gamma^{\prime}$ & & $\mathrm{C}=\mathrm{O}$ \\
\hline$\gamma$ & $17.4, \mathrm{CH}_{3}$ & $0.76, \mathrm{~d}(6.7)$ & $\beta$ & $\beta, \gamma^{\prime}$ & & $\alpha, \beta, \gamma^{\prime}$ \\
\hline$\gamma^{\prime}$ & $19.4, \mathrm{CH}_{3}$ & $0.81, \mathrm{~d}(6.7)$ & $\beta$ & $\beta$ & & $\alpha, \beta, \gamma$ \\
\hline $\mathrm{NH}$ & & $7.80, \mathrm{~m}^{\mathrm{b}}$ & $\alpha$ & $\alpha, \beta$ & Pro $\alpha$ & \\
\hline $\mathrm{CO}$ & $171.9, \mathrm{C}$ & & & & & \\
\hline$t$-Leu $\alpha$ & $60.2, \mathrm{CH}$ & $4.13, \mathrm{~m}^{\mathrm{b}}$ & $\mathrm{NH}$ & $\mathrm{NH}$ & Dioia-NH & $\beta, \gamma, \mathrm{C}=\mathrm{O}$ \\
\hline$\beta$ & $34.1, \mathrm{C}$ & & & & & \\
\hline$\gamma$ & $26.6, \mathrm{CH}_{3}$ & $0.86, \mathrm{~s}$ & & & & $\alpha, \beta$ \\
\hline $\mathrm{NH}$ & & $7.82, \mathrm{~m}^{\mathrm{b}}$ & $\alpha$ & $\alpha$ & Val $\alpha$ & \\
\hline $\mathrm{CO}$ & $170.0, \mathrm{C}$ & & & & & \\
\hline Dioia $\alpha$ & $48.5, \mathrm{CH}$ & $4.46, \mathrm{~m}^{\mathrm{b}}$ & $\mathrm{NH}, \beta 1, \beta 2$ & $\mathrm{NH}, \beta 1, \beta 2$ & Arg-NH & $\mathrm{C}=\mathrm{O}$ \\
\hline$\beta 1$ & $39.0, \mathrm{CH}_{2}$ & $2.30, \mathrm{~m}$ & $\alpha, \beta 2$ & $\mathrm{NH}, \alpha$ & & 2,3 \\
\hline$\beta 2$ & & $1.89, \mathrm{~m}$ & $\alpha, \beta 1$ & $\mathrm{NH}, \alpha$ & & 2,3 \\
\hline $\mathrm{C} 2$ & $178.2, \mathrm{C}$ & & & & & \\
\hline $\mathrm{C} 3$ & $74.0, \mathrm{C}$ & & & & & \\
\hline $\mathrm{C} 3 \mathrm{a}$ & $131.7, \mathrm{C}$ & & & & & \\
\hline $\mathrm{C} 7 \mathrm{a}$ & $141.7, \mathrm{C}$ & & & & & \\
\hline $\mathrm{C} 4$ & $124.0, \mathrm{CH}$ & $7.35, \mathrm{~d}(7.6)$ & 5 & $5,6,7$ & & $3,7 \mathrm{a}$ \\
\hline $\mathrm{C} 5$ & $122.0, \mathrm{CH}$ & 6.90, dd $(7.6,7.3)$ & 4,6 & $4,6,7$ & & $3 a, 7$ \\
\hline C6 & $128.0, \mathrm{CH}$ & $7.18, \mathrm{~m}^{\mathrm{b}}$ & 5,7 & $4,5,7$ & & $7 \mathrm{a}$ \\
\hline $\mathrm{C} 7$ & $109.8, \mathrm{CH}$ & $6.78, \mathrm{~d}(8.0)$ & 6 & $4,5,6$ & & $3 a, 5$ \\
\hline $1-\mathrm{NH}$ & & 10.24 , brs & & & & 3 \\
\hline $3-\mathrm{OH}$ & & 6.16 , brs & & & & \\
\hline $\mathrm{NH}$ & & 8.21, brd (7.9) & $\alpha$ & & $t$-Leu $\alpha$ & \\
\hline $\mathrm{CO}$ & $169.8, \mathrm{C}$ & & & & & \\
\hline $\operatorname{Arg} \alpha$ & $52.0, \mathrm{CH}$ & $4.22, \mathrm{~m}^{\mathrm{b}}$ & $\mathrm{NH}, \beta$ & $\mathrm{NH}, \beta$ & $\mathrm{Cys}\left(\mathrm{SO}_{3} \mathrm{H}\right)-\mathrm{NH}$ & \\
\hline$\beta$ & $29.1, \mathrm{CH}_{2}$ & $1.65, \mathrm{~m}$ & $\alpha, \gamma$ & $\alpha, \gamma, \delta$ & & $\mathrm{C}=\mathrm{O}$ \\
\hline$\gamma$ & $23.2, \mathrm{CH}_{2}$ & $1.40, \mathrm{~m}$ & $\beta, \delta$ & $\alpha, \beta, \delta$ & & \\
\hline$\delta$ & $40.2, \mathrm{CH}_{2}$ & $3.01, \mathrm{~m}^{\mathrm{b}}$ & $\gamma$ & $\beta, \gamma$ & & \\
\hline guanidine & $156.8, \mathrm{C}$ & & & & & \\
\hline $\mathrm{NH}$ & & 7.81, brs $^{b}$ & $\alpha$ & $\alpha$ & Dioia $\alpha$ & Dioia $\mathrm{C}=\mathrm{O}$ \\
\hline $\mathrm{CO}$ & $171.0, \mathrm{C}$ & & & & & \\
\hline $\mathrm{Cys}\left(\mathrm{SO}_{3} \mathrm{H}\right) \alpha$ & $50.9, \mathrm{CH}$ & $4.59, \mathrm{~m}$ & $\mathrm{NH}, \beta$ & $\mathrm{NH}, \beta$ & Thr1-NH & $\mathrm{C}=\mathrm{O}$ \\
\hline$\beta$ & $52.5, \mathrm{CH}_{2}$ & $2.90, \mathrm{~m}$ & $\alpha$ & $\alpha$ & & \\
\hline $\mathrm{NH}$ & & 8.30, brd $(8.3)$ & $\alpha$ & $\alpha, \beta$ & $\operatorname{Arg} \alpha$ & $\operatorname{Arg} \mathrm{C}=\mathrm{O}$ \\
\hline $\mathrm{CO}$ & $169.4, \mathrm{C}$ & & & & & \\
\hline Thr1 $\alpha$ & $51.9, \mathrm{CH}$ & $4.84, \mathrm{~d}(8.5)$ & $\mathrm{NH}$ & $\mathrm{NH}$ & NMeGln-NMe & $\gamma, \mathrm{C}=\mathrm{O}, \mathrm{Cys}\left(\mathrm{SO}_{3} \mathrm{H}\right) \mathrm{C}=\mathrm{O}$ \\
\hline$\beta$ & $69.2, \mathrm{CH}$ & $5.14, \mathrm{~d}(6.7)$ & $\gamma$ & $\gamma$ & & $\alpha, \gamma, \operatorname{Sar} C=\mathrm{O}$ \\
\hline$\gamma$ & $17.4, \mathrm{CH}_{3}$ & $1.17, \mathrm{~d}(6.5)$ & $\beta$ & $\beta$ & & $\alpha$ \\
\hline $\mathrm{NH}$ & & $7.96, \mathrm{~m}$ & $\alpha$ & $\alpha$ & $\mathrm{Cys}\left(\mathrm{SO}_{3} \mathrm{H}\right) \alpha$ & \\
\hline $\mathrm{CO}$ & $169.2, \mathrm{C}$ & & & & & \\
\hline $\mathrm{NMeG} \ln \alpha$ & $54.5, \mathrm{CH}$ & $4.98, \mathrm{~m}^{\mathrm{b}}$ & $\beta$ & $\beta, \gamma$ & $\beta, \gamma$, Phe-NH & $\mathrm{C}=\mathrm{O}, \mathrm{NMe}$ \\
\hline$\beta$ & 24.7, $\mathrm{CH}_{2}$ & $1.70, \mathrm{~m}$ & $\alpha, \gamma$ & $\alpha, \gamma$ & $\alpha, \gamma$ & $\alpha$ \\
\hline$\gamma$ & $31.5, \mathrm{CH}_{2}$ & $1.89, \mathrm{~m}$ & $\beta$ & $\alpha, \beta$ & & $\mathrm{CONH}_{2}$ \\
\hline $\mathrm{NMe}$ & $30.4, \mathrm{CH}_{3}$ & $2.94, \mathrm{~s}$ & & & $\alpha$, Thr1 $\alpha, \operatorname{Thr} 1 \beta$ & $\alpha, \operatorname{Thr} 1 \mathrm{C}=\mathrm{O}$ \\
\hline \multirow[t]{2}{*}{$\mathrm{CONH}_{2}$} & $173.5, \mathrm{C}$ & $6.64, \mathrm{brs}$ & & & & $\gamma$ \\
\hline & & $7.17, \mathrm{brs}^{\mathrm{b}}$ & & & & $\gamma$ \\
\hline $\mathrm{CO}$ & $167.5, \mathrm{C}$ & & & & & \\
\hline Phe $\alpha$ & $54.0, \mathrm{CH}$ & $4.69, \mathrm{~d}(7.2)$ & $\mathrm{NH}, \beta$ & $\mathrm{NH}, \beta$ & Thr2-NH & $\beta, C=O$ \\
\hline
\end{tabular}




\begin{tabular}{|c|c|c|c|c|c|c|}
\hline $\begin{array}{l}\beta \\
\mathrm{C} 1\end{array}$ & $\begin{array}{l}37.9, \mathrm{CH}_{2} \\
137.1, \mathrm{C}\end{array}$ & $2.98, \mathrm{~m}$ & $\alpha$ & $\mathrm{NH}, \alpha$ & & $\alpha$ \\
\hline $\mathrm{C} 2 / \mathrm{C} 6$ & $129.3, \mathrm{CH}$ & $7.18, \mathrm{~m}^{\mathrm{b}}$ & 3,5 & $3,4,5$ & & $\beta, 4$ \\
\hline $\mathrm{C} 3 / \mathrm{C} 5$ & $129.0, \mathrm{CH}$ & $7.23, \mathrm{~m}^{\mathrm{b}}$ & $2,4,6$ & $2,4,6$ & & $1,2,6$ \\
\hline $\mathrm{C} 4$ & $126.2, \mathrm{CH}$ & $7.15, \mathrm{~m}^{\mathrm{b}}$ & 3,5 & $2,3,5,6$ & & $2,3,5,6$ \\
\hline $\mathrm{NH}$ & & 7.56, brd (6.6) & $\alpha$ & $\alpha, \beta$ & $\mathrm{NMeGln} \alpha$ & \\
\hline $\mathrm{CO}$ & $170.7, \mathrm{C}$ & & & & & \\
\hline Thr $2 \alpha$ & $60.7, \mathrm{CH}$ & $3.85, \mathrm{~m}$ & $\mathrm{NH}, \beta$ & $\mathrm{NH}, \beta, \gamma, \mathrm{OH}$ & Asn-NH & $\beta, C=O$ \\
\hline$\beta$ & $65.5, \mathrm{CH}$ & $3.96, \mathrm{~m}$ & $\alpha, \gamma, \mathrm{OH}$ & $\gamma, \mathrm{NH}, \mathrm{OH}$ & Asn-NH & \\
\hline $\begin{array}{l}\gamma \\
\mathrm{OH}\end{array}$ & $20.1, \mathrm{CH}_{3}$ & $\begin{array}{l}0.89, \mathrm{~d}(6.3) \\
4.88, \mathrm{~d}(4.9)\end{array}$ & $\beta$ & $\alpha, \beta, \mathrm{OH}$ & & $\alpha, \beta$ \\
\hline $\mathrm{NH}$ & & 7.95, brs & $\alpha$ & $\alpha, \beta$ & Phe $\alpha$ & \\
\hline $\mathrm{CO}$ & $169.6, \mathrm{C}$ & & & & & \\
\hline Asn $\alpha$ & $46.0, \mathrm{CH}$ & $4.98, \mathrm{~m}^{\mathrm{b}}$ & $\mathrm{NH}, \beta 1, \beta 2$ & $\mathrm{NH}, \alpha, \beta 1, \beta 2$ & & $\mathrm{CONH}_{2}$ \\
\hline$\beta 1$ & $36.9, \mathrm{CH}_{2}$ & $2.09, \mathrm{~m}$ & $\alpha$ & $\mathrm{NH}, \alpha, \beta 1, \beta 2$ & & $\mathrm{C}=\mathrm{O}, \mathrm{CONH}_{2}$ \\
\hline$\beta 2$ & & $2.30, \mathrm{~m}$ & $\alpha$ & $\mathrm{NH}, \alpha, \beta 1, \beta 2$ & & $\alpha, \mathrm{CONH}_{2}$ \\
\hline $\mathrm{CONH}_{2}$ & 172.1, C & $\begin{array}{l}6.72 \text {, brs } \\
7.33, \text { brs }\end{array}$ & & & & $\mathrm{CONH}_{2}$ \\
\hline NH & & 7.51, brd (7.6) & $\alpha$ & $\mathrm{NH}, \alpha, \beta 1, \beta 2$ & $\operatorname{Thr} 2 \alpha, \operatorname{Thr} 2 \beta$ & $\mathrm{C}=\mathrm{O}$ \\
\hline $\mathrm{CO}$ & $169.4, \mathrm{C}$ & & & & & \\
\hline $\operatorname{Sar} \alpha 1$ & $49.5, \mathrm{CH}_{2}$ & $3.49, \mathrm{~d}(17.3)$ & $\alpha 2$ & $\alpha 2$ & & $\mathrm{C}=\mathrm{O}, \mathrm{Asn} \mathrm{C}=\mathrm{O}$ \\
\hline$\alpha 2$ & & $4.42, \mathrm{~m}^{\mathrm{b}}$ & $\alpha 1$ & $\alpha 1$ & & $\mathrm{C}=\mathrm{O}$ \\
\hline $\mathrm{NMe}$ & $35.4, \mathrm{CH}_{3}$ & $2.74, \mathrm{~s}$ & & & & $\alpha$ \\
\hline $\mathrm{CO}$ & $167.5, \mathrm{C}$ & & & & & \\
\hline
\end{tabular}

${ }^{a}$ Derived from the combination of ${ }^{13} \mathrm{C}$ NMR, HSQC and HMBC data. ${ }^{b}$ Singals overlapped. 
Table S4. NMR spectroscopic data for halicylindramide H (3) in DMSO- $d_{6}$ at $600 \mathrm{MHz}\left({ }^{1} \mathrm{H}\right)$ and $150 \mathrm{MHz}\left({ }^{13} \mathrm{C}\right)$

\begin{tabular}{|c|c|c|c|c|c|c|}
\hline position & $\delta \mathrm{c}$, mult. $^{\mathrm{a}}$ & $\delta_{\mathrm{H}},(\mathrm{J}$ in $\mathrm{Hz})$ & COSY & TOCSY & NOESY & HMBC (H to C) \\
\hline $\mathrm{NCHO}$ & $161.4, \mathrm{C}$ & $7.90, \mathrm{~s}$ & Ala-NH & Ala-NH, Ala $\alpha$, Ala $\beta$ & Ala-NH & \\
\hline Ala $\alpha$ & $47.6, \mathrm{CH}$ & $4.31, \mathrm{~m}^{\mathrm{b}}$ & $\mathrm{NH}, \beta$ & $\mathrm{NH}, \beta$ & $\beta$, Br-Phe-NH & $\beta$ \\
\hline$\beta$ & $19.5, \mathrm{CH}_{3}$ & $0.92, \mathrm{~d}(7.0)$ & $\alpha$ & $\mathrm{NH}, \alpha$ & & $\alpha, \mathrm{C}=\mathrm{O}$ \\
\hline NH & & 8.18, brs & formyl, $\alpha$ & formyl, $\alpha, \beta$ & formyl & \\
\hline $\mathrm{CO}$ & $172.3, \mathrm{C}$ & & & & & \\
\hline BrPhe $\alpha$ & $52.6, \mathrm{CH}$ & $4.72, \mathrm{~m}^{\mathrm{b}}$ & $\mathrm{NH}, \beta 1, \beta 2$ & $\mathrm{NH}, \beta 1, \beta 2$ & Pro $\delta$ & $\beta, 1$ \\
\hline$\beta 1$ & $37.1, \mathrm{CH}_{2}$ & $2.71, \mathrm{~m}$ & $\beta 2$ & $\mathrm{NH}, \alpha, \beta 2$ & $\alpha$ & $\alpha, \mathrm{C}=\mathrm{O}, 1,2,6$ \\
\hline$\beta 2$ & & $3.01, \mathrm{~m}^{\mathrm{b}}$ & $\alpha, \beta 1$ & $\mathrm{NH}, \alpha, \beta 1$ & $\alpha, \operatorname{Pro} \delta$ & $\alpha, \mathrm{C}=\mathrm{O}, 1,2,6$ \\
\hline $\mathrm{C} 1$ & $138.2, \mathrm{C}$ & & & & & \\
\hline $\mathrm{C} 2 / \mathrm{C} 6$ & $132.6, \mathrm{CH}$ & $7.26, \mathrm{~m}^{\mathrm{b}}$ & 3,5 & 3,5 & $\beta 1, \beta 2$, Ala $\beta 1$, Ala $\beta 2$ & $\beta, 1,3,4,6$ \\
\hline $\mathrm{C} 3 / \mathrm{C} 5$ & $131.7, \mathrm{CH}$ & $7.43, \mathrm{~d}(8.1)$ & 2,6 & 2,6 & $\beta 1, \beta 2$, Ala $\beta 1$, Ala $\beta 2$, & $1,2,4,5$ \\
\hline $\mathrm{C} 4$ & $120.3, \mathrm{C}$ & & & & & \\
\hline NH & & $8.42, \mathrm{~m}^{\mathrm{b}}$ & $\alpha$ & $\alpha, \beta 1, \beta 2$ & Ala $\alpha$ & $\mathrm{C}=\mathrm{O}$ \\
\hline $\mathrm{CO}$ & $171.4, \mathrm{C}$ & & & & & \\
\hline Pro $\alpha$ & $59.8, \mathrm{C}$ & $4.40, \mathrm{~m}^{\mathrm{b}}$ & $\beta 1, \beta 2$ & $\beta 1, \beta 2, \gamma$ & Val-NH & $\beta, \gamma$ \\
\hline$\beta 1$ & $30.5, \mathrm{CH}_{2}$ & $1.80, \mathrm{~m}^{\mathrm{b}}$ & $\alpha, \gamma$ & $\alpha, \beta 2, \gamma, \delta$ & & $\alpha, \gamma, \delta, C=O$ \\
\hline$\beta 2$ & & $2.02, \mathrm{~m}^{\mathrm{b}}$ & $\alpha, \gamma$ & $\alpha, \beta 1, \gamma, \delta$ & & $\mathrm{C}=\mathrm{O}$ \\
\hline$\gamma$ & $25.2, \mathrm{CH}_{2}$ & $1.83, \mathrm{~m}^{\mathrm{b}}$ & $\beta 1, \beta 2, \delta 1, \delta 2$ & $\alpha, \beta 1, \beta 2, \delta 1, \delta 2$ & $\alpha, \delta$ & $\alpha, \beta, \delta$ \\
\hline$\delta 1$ & $47.8, \mathrm{CH}_{2}$ & $3.60, \mathrm{~m}$ & $\gamma$ & $\beta, \gamma$ & BrPhe $\alpha$, BrPhe $\beta 2$ & $\beta, \gamma$ \\
\hline$\delta 2$ & & $3.71, \mathrm{~m}$ & $\gamma$ & $\beta, \gamma$ & & $\beta, \gamma$ \\
\hline $\mathrm{CO}$ & $168.7, \mathrm{C}$ & & & & & \\
\hline Val $\alpha$ & $58.1, \mathrm{CH}$ & $4.37, \mathrm{~m}^{\mathrm{b}}$ & $\mathrm{NH}, \beta$ & $\mathrm{NH}, \beta, \gamma, \gamma^{\prime}$ & $t$-Leu-NH & $\beta, \gamma, \gamma^{\prime}, \mathrm{C}=\mathrm{O}$ \\
\hline$\beta$ & $31.8, \mathrm{CH}$ & $2.09, \mathrm{~m}^{\mathrm{b}}$ & $\alpha, \gamma, \gamma^{\prime}$ & $\mathrm{NH}, \alpha, \gamma, \gamma^{\prime}$ & & $\alpha, \gamma, \gamma^{\prime}, \mathrm{C}=\mathrm{O}$ \\
\hline$\gamma$ & $18.2, \mathrm{CH}_{3}$ & $0.77, \mathrm{~d}(6.7)$ & $\beta$ & $\alpha, \beta, \gamma^{\prime}$ & & $\alpha, \beta, \gamma^{\prime}$ \\
\hline$\gamma^{\prime}$ & $20.3, \mathrm{CH}_{3}$ & $0.82, \mathrm{~d}(6.5)$ & $\beta$ & $\alpha, \beta, \gamma$ & & $\alpha, \beta, \gamma$ \\
\hline $\mathrm{NH}$ & & $7.78, \mathrm{~m}^{\mathrm{b}}$ & $\alpha$ & $\alpha, \beta$ & Pro $\alpha$ & \\
\hline $\mathrm{CO}$ & $171.4, \mathrm{C}$ & & & & & \\
\hline$t$-Leu $\alpha$ & $61.3, \mathrm{CH}$ & $4.13, \mathrm{~m}^{\mathrm{b}}$ & $\mathrm{NH}$ & $\mathrm{NH}$ & Dioia-NH & $\gamma, \mathrm{C}=\mathrm{O}$ \\
\hline$\beta$ & $34.9, \mathrm{C}$ & & & & & \\
\hline$\gamma$ & $27.6, \mathrm{CH}_{3}$ & $0.89, \mathrm{~s}$ & & & Dioia-NH & $\alpha, \beta$ \\
\hline $\mathrm{NH}$ & & $7.91, \mathrm{~m}^{\mathrm{b}}$ & $\alpha$ & $\alpha$ & Val $\alpha$ & \\
\hline $\mathrm{CO}$ & $170.9, \mathrm{C}$ & & & & & \\
\hline Dioia $\alpha$ & $50.8, \mathrm{CH}$ & $4.42, \mathrm{~m}^{\mathrm{b}}$ & $\mathrm{NH}$ & $\mathrm{NH}, \beta 1, \beta 2$ & Arg-NH & $\beta, 3, \mathrm{C}=\mathrm{O}$ \\
\hline$\beta 1$ & $39.9, \mathrm{CH}_{2}$ & $2.18, \mathrm{~m}$ & $\alpha$ & $\mathrm{NH}, \alpha$ & & $\alpha, 3$ \\
\hline$\beta 2$ & & $1.96, \mathrm{~m}$ & $\alpha$ & $\mathrm{NH}, \alpha$ & & $\alpha, 2,3$ \\
\hline $\mathrm{C} 2$ & $179.8, \mathrm{C}$ & & & & & \\
\hline $\mathrm{C} 3$ & $75.2, \mathrm{C}$ & & & & & \\
\hline $\mathrm{C} 3 \mathrm{a}$ & 132.0, C & & & & & \\
\hline $\mathrm{C} 7 \mathrm{a}$ & 142.1, C & & & & & \\
\hline $\mathrm{C} 4$ & $125.0, \mathrm{CH}$ & $7.23, \mathrm{~m}^{\mathrm{b}}$ & 5 & $5,6,7$ & 5 & $3 a, 6,7 a$ \\
\hline C5 & $122.6, \mathrm{CH}$ & 6.94, brt $(7.3)$ & 4,6 & $4,6,7$ & 4,6 & $3 a, 7$ \\
\hline C6 & $129.3, \mathrm{CH}$ & $7.19, \mathrm{~m}^{\mathrm{b}}$ & 5,7 & $4,5,7$ & 5,7 & $4,7 \mathrm{a}$ \\
\hline $\mathrm{C} 7$ & $110.8, \mathrm{CH}$ & 6.80, brd $(7.5)$ & 6 & $4,5,6$ & 6 & $3 a, 5$ \\
\hline $1-\mathrm{NH}$ & & 10.32, brs & & & & 3 \\
\hline $3-\mathrm{OH}$ & & 6.18, brs & & & $\alpha, \beta 1, \beta 2$ & \\
\hline $\mathrm{NH}$ & & 8.33 , brs & $\alpha$ & $\alpha, \beta 1, \beta 2$ & $\beta, t$-Leu $\alpha, t$-Leu $\gamma$ & \\
\hline $\mathrm{CO}$ & $169.1, \mathrm{C}$ & & & & & \\
\hline $\operatorname{Arg} \alpha$ & $53.0, \mathrm{CH}$ & $4.27, \mathrm{~m}^{\mathrm{b}}$ & $\mathrm{NH}, \beta$ & $\mathrm{NH}, \beta$ & $\mathrm{Cys}\left(\mathrm{SO}_{3} \mathrm{H}\right)-\mathrm{NH}$ & $\beta$ \\
\hline$\beta$ & $29.5, \mathrm{CH}_{2}$ & $1.68, \mathrm{~m}^{\mathrm{b}}$ & $\alpha, \gamma$ & $\alpha, \gamma$ & & $\alpha, \mathrm{C}=\mathrm{O}$ \\
\hline$\gamma$ & $24.8, \mathrm{CH}_{2}$ & $1.45, \mathrm{~m}$ & $\beta, \delta$ & $\beta, \delta$ & & $\beta, \delta$ \\
\hline$\delta$ & $41.2, \mathrm{CH}_{2}$ & $3.01, \mathrm{~m}^{\mathrm{b}}$ & $\gamma$ & $\beta, \gamma$ & & $\beta, \gamma$ \\
\hline guanidine & $157.7, \mathrm{C}$ & & & & & \\
\hline $\mathrm{NH}$ & & $7.82, \mathrm{brs}^{\mathrm{b}}$ & $\alpha$ & $\alpha$ & & Dioia $\mathrm{C}=\mathrm{O}$ \\
\hline $\mathrm{CO}$ & $172.3, \mathrm{C}$ & & & & & \\
\hline $\mathrm{Cys}\left(\mathrm{SO}_{3} \mathrm{H}\right) \alpha$ & $51.8, \mathrm{CH}$ & $4.59, \mathrm{~m}$ & $\mathrm{NH}, \beta$ & $\mathrm{NH}, \beta$ & Thr1-NH, Thr1- $\gamma$ & $\beta, C=O$ \\
\hline$\beta$ & $53.4, \mathrm{CH}_{2}$ & $2.96, \mathrm{~m}^{\mathrm{b}}$ & $\alpha$ & $\mathrm{NH}, \alpha$ & $\mathrm{NH}$ & $\alpha$ \\
\hline $\mathrm{NH}$ & & 8.30, brd $(7.2)$ & $\alpha$ & $\alpha, \beta$ & $\beta, \operatorname{Arg} \alpha$ & $\operatorname{Arg} \mathrm{C}=\mathrm{O}$ \\
\hline $\mathrm{CO}$ & 169.0, C & & & & & \\
\hline Thr1 $\alpha$ & $52.8, \mathrm{CH}$ & $4.83, \mathrm{~d}(8.6)$ & $\mathrm{NH}$ & NH & NMeGln-NMe, NMeGln $\gamma$ & $\gamma, \mathrm{C}=\mathrm{O}, \mathrm{Cys}(\mathrm{SO} 3 \mathrm{H}) \mathrm{C}=\mathrm{O}$ \\
\hline$\beta$ & $69.8, \mathrm{CH}$ & 5.13 , brq $(6.6)$ & $\alpha, \gamma$ & $\gamma$ & NMeGln-NMe & $\gamma, \mathrm{Sar} C=\mathrm{O}$ \\
\hline$\gamma$ & $18.2, \mathrm{CH}_{3}$ & $1.16, \mathrm{~d}(6.5)$ & $\beta$ & $\beta$ & $\mathrm{NH}, \alpha, \beta, \mathrm{Cys}\left(\mathrm{SO}_{3} \mathrm{H}\right) \alpha$ & $\alpha, \beta$ \\
\hline $\mathrm{NH}$ & & 8.01 , brs & $\alpha$ & $\alpha$ & $\gamma, \mathrm{Cys}\left(\mathrm{SO}_{3} \mathrm{H}\right) \alpha, \mathrm{Cys}\left(\mathrm{so}_{\mathrm{H}}\right) \beta$ & \\
\hline $\mathrm{CO}$ & $170.5, \mathrm{C}$ & & & & & \\
\hline $\mathrm{NMeG} \ln \alpha$ & $55.2, \mathrm{CH}$ & $4.96, \mathrm{~m}^{\mathrm{b}}$ & $\beta 1, \beta 2$ & $\beta 1, \beta 2, \gamma 1, \gamma 2$ & $\gamma$, Thr1 $\gamma$, Phe-NH & $\mathrm{C}=\mathrm{O}, \mathrm{NMe}$ \\
\hline$\beta 1$ & 26.7, $\mathrm{CH}_{2}$ & $1.70, \mathrm{~m}^{\mathrm{b}}$ & $\alpha, \gamma 2$ & $\alpha, \beta 2, \gamma 1, \gamma 2$ & & $\gamma$ \\
\hline$\beta 2$ & & $1.89, \mathrm{~m}^{\mathrm{b}}$ & $\alpha, \gamma 2$ & $\alpha, \beta 1, \gamma 1, \gamma 2$ & & \\
\hline$\gamma 1$ & $32.4, \mathrm{CH}_{2}$ & $1.87, \mathrm{~m}^{\mathrm{b}}$ & $\beta 1, \beta 2, \gamma 2$ & $\alpha, \beta 1, \beta 2, \gamma 2$ & Thr1 $\alpha$ & $\mathrm{CONH}_{2}$ \\
\hline
\end{tabular}




\begin{tabular}{|c|c|c|c|c|c|c|}
\hline$\gamma^{2}$ & & $1.92, \mathrm{~m}^{\mathrm{b}}$ & $\beta 1, \beta 2, \gamma 1$ & $\alpha, \beta 1, \beta 2, \gamma 1$ & & \\
\hline $\mathrm{NMe}$ & $31.3, \mathrm{CH}_{3}$ & $2.94, \mathrm{~s}$ & & & $\operatorname{Thr} 1 \alpha, \operatorname{Thr} 1 \beta$ & $\alpha$, Thr1 C $=\mathrm{O}$ \\
\hline $\mathrm{CONH}_{2}$ & $174.4, \mathrm{C}$ & $\begin{array}{l}\text { 6.67, brs } \\
7.16, \mathrm{brs}^{\mathrm{b}}\end{array}$ & & & & $\begin{array}{l}\gamma \\
\gamma\end{array}$ \\
\hline $\mathrm{CO}$ & $168.6, \mathrm{C}$ & & & & & \\
\hline Phe $\alpha$ & $55.0, \mathrm{CH}$ & $4.69, \mathrm{~m}^{\mathrm{b}}$ & $\mathrm{NH}, \beta 1, \beta 2$ & $\mathrm{NH}, \beta 1, \beta 2$ & Phe-NH, Thr2-NH & $\beta$ \\
\hline$\beta 1$ & $39.2, \mathrm{CH}_{2}$ & $2.96, \mathrm{~m}^{\mathrm{b}}$ & $\alpha$ & $\mathrm{NH}, \alpha$ & Thr2-NH & $\mathrm{C}=\mathrm{O}, 1,2,6$ \\
\hline$\beta 2$ & & $3.02, \mathrm{~m}^{\mathrm{b}}$ & $\alpha$ & $\mathrm{NH}, \alpha$ & & \\
\hline $\mathrm{C} 1$ & $138.0, \mathrm{C}$ & & & & & \\
\hline $\mathrm{C} 2 / \mathrm{C} 6$ & $130.2, \mathrm{CH}$ & $7.18, \mathrm{~m}^{\mathrm{b}}$ & 3,5 & $3,4,5$ & $\beta 1, \beta 2, \mathrm{NMeGln} \gamma, \operatorname{Thr} 2 \gamma$ & $\beta, 4$ \\
\hline $\mathrm{C} 3 / \mathrm{C} 5$ & $128.9, \mathrm{CH}$ & $7.23, \mathrm{~m}^{\mathrm{b}}$ & $2,4,6$ & $2,4,6$ & $\beta 1, \beta 2$ & 1 \\
\hline $\mathrm{C} 4$ & $127.2, \mathrm{CH}$ & $7.17, \mathrm{~m}^{\mathrm{b}}$ & 3,5 & $2,3,5,6$ & & 2,6 \\
\hline $\mathrm{NH}$ & & 7.56 , brs & & & $\mathrm{NMeGln} \alpha$ & \\
\hline $\mathrm{CO}$ & $171.9, \mathrm{C}$ & & & & & \\
\hline Thr $2 \alpha$ & $61.9, \mathrm{CH}$ & $3.86, \mathrm{~m}$ & $\mathrm{NH}, \beta$ & $\mathrm{NH}, \beta, \gamma, \mathrm{OH}$ & Asn-NH & Phe $\mathrm{C}=\mathrm{O}$ \\
\hline$\beta$ & $66.3, \mathrm{CH}$ & 3.96 , brs & $\alpha, \gamma$ & $\alpha, \gamma, \mathrm{OH}$ & & \\
\hline$\gamma$ & $21.0, \mathrm{CH}_{3}$ & $0.85, \mathrm{~m}^{\mathrm{b}}$ & $\beta$ & $\alpha, \beta, \mathrm{OH}$ & Asn-NH & $\alpha, \beta$ \\
\hline $\mathrm{OH}$ & & $4.90, \mathrm{brs}$ & & & & \\
\hline $\mathrm{NH}$ & & 7.96, brd $(6.8)$ & $\alpha$ & $\alpha, \beta$ & $\gamma$, Phe $\alpha$, Phe $\beta$ & \\
\hline $\mathrm{CO}$ & $170.1, \mathrm{C}$ & & & & & \\
\hline Asn $\alpha$ & $46.8, \mathrm{CH}$ & $4.99, \mathrm{~m}^{\mathrm{b}}$ & $\mathrm{NH}, \beta 1, \beta 2$ & $\mathrm{NH}, \alpha, \beta 1, \beta 2$ & Sar-NMe & $\mathrm{CONH}_{2}$ \\
\hline$\beta 1$ & $38.1, \mathrm{CH}_{2}$ & $2.08, \mathrm{~m}^{\mathrm{b}}$ & $\alpha$ & $\mathrm{NH}, \alpha, \beta 1, \beta 2$ & & $\mathrm{C}=\mathrm{O}, \mathrm{CONH}_{2}$ \\
\hline$\beta 2$ & & $2.66, \mathrm{~m}^{\mathrm{b}}$ & $\alpha$ & $\mathrm{NH}, \alpha, \beta 1, \beta 2$ & & $\alpha, \mathrm{CONH}_{2}$ \\
\hline & & 7.50, brd $(9.1)$ & $\alpha$ & $\mathrm{NH}, \alpha, \beta 1, \beta 2$ & $\beta 1, \operatorname{Thr} 2 \alpha, \operatorname{Thr} 2 \gamma$, Sar-NMe, & $\mathrm{C}=\mathrm{O}$ \\
\hline $\mathrm{CONH}_{2}$ & 173.0, C & 6.77, brs & & & $\beta 1, \beta 2$ & $\beta, \mathrm{CONH}_{2}$ \\
\hline & & 7.32 , brs & & & $\beta 1, \beta 2$ & $\beta$ \\
\hline $\mathrm{CO}$ & $170.2, \mathrm{C}$ & & & & & \\
\hline Sar $\alpha 1$ & $50.6, \mathrm{CH}_{2}$ & $3.48, \mathrm{~m}^{\mathrm{b}}$ & $\alpha 2$ & $\alpha 2$ & & $\mathrm{C}=\mathrm{O}, \mathrm{Asn} \mathrm{C}=\mathrm{O}$ \\
\hline$\alpha 2$ & & $4.42, \mathrm{~m}^{\mathrm{b}}$ & $\alpha 1$ & $\alpha 1$ & & $\mathrm{C}=\mathrm{O}$ \\
\hline $\mathrm{NMe}$ & $36.4, \mathrm{CH}_{3}$ & $2.73, \mathrm{~s}$ & & & & $\alpha, A \operatorname{sn} C=\mathrm{O}$ \\
\hline $\mathrm{CO}$ & $168.5, \mathrm{C}$ & & & & & \\
\hline
\end{tabular}

${ }^{a}$ Derived from the combination of ${ }^{13} \mathrm{C}$ NMR, HSQC and HMBC data. ${ }^{b}$ Singals overlapped. 
Table S5. Absolute configuration of halicylindramides G (2) and H (3) by advanced Marfey's method and Edman degradation

\begin{tabular}{|c|c|c|c|c|c|}
\hline \multirow[t]{2}{*}{ Parent peptide } & \multirow{2}{*}{$\begin{array}{l}\text { Constitutive } \\
\text { amino acids }\end{array}$} & \multirow[t]{2}{*}{$m / z$} & \multicolumn{2}{|c|}{ Retention time } & \multirow{2}{*}{$\begin{array}{c}\text { Absolute } \\
\text { configuration of } \\
\text { amino acids } \\
\end{array}$} \\
\hline & & & AA-L-DLA & AA-D-DLA & \\
\hline \multirow[t]{11}{*}{2} & $\mathrm{Ala}^{a}$ & $495-496$ & 38.40 & 34.49 & $\mathrm{D}$ \\
\hline & BrPhe $^{a}$ & 648.5-649.5 & 48.32 & 55.27 & $\mathrm{~L}$ \\
\hline & Pro $^{a}$ & 521.5-522.5 & 33.71 & 37.28 & $\mathrm{~L}$ \\
\hline & $\mathrm{Val}^{a}$ & $523-524$ & 46.90 & 38.97 & $\mathrm{D}$ \\
\hline & $t-\mathrm{Leu}^{a}$ & $537-538$ & 41.42 & 50.39 & $\mathrm{~L}$ \\
\hline & $\operatorname{Arg}^{b, c}$ & 694-695 & 25.98 & 21.32 & $\mathrm{~L}$ \\
\hline & $\mathrm{Cys}\left(\mathrm{SO}_{3} \mathrm{H}\right)^{a, c}$ & $575-576$ & 11.66 & 12.74 & $\mathrm{D}$ \\
\hline & Thr1, Thr2 ${ }^{a}$ & $525-526$ & 27.73 & 33.90 & $\mathrm{~L}$ \\
\hline & $\mathrm{NMeGln}^{a}$ & $567-568$ & 28.75 & 29.69 & $\mathrm{~L}$ \\
\hline & Phe $^{d}$ & $457.5-458.8$ & 43.50 & 49.38 & $\mathrm{D}$ \\
\hline & $\mathrm{Asn}^{d}$ & $524.5-525.0$ & 36.31 & 36.31 & \\
\hline \multirow[t]{11}{*}{3} & $\mathrm{Ala}^{a}$ & 495-496 & 38.27 & 34.37 & $\mathrm{D}$ \\
\hline & BrPhe $^{a}$ & 648.5-649.5 & 48.17 & 55.11 & $\mathrm{~L}$ \\
\hline & Pro $^{a}$ & $521.5-522.5$ & 33.59 & 37.16 & $\mathrm{~L}$ \\
\hline & $\mathrm{Val}^{a}$ & $523-524$ & 46.76 & 38.84 & $\mathrm{D}$ \\
\hline & $t-\mathrm{Leu}^{a}$ & $537-538$ & 41.29 & 50.24 & $\mathrm{~L}$ \\
\hline & $\mathrm{Arg}^{b, c}$ & 694-695 & 25.88 & 21.23 & $\mathrm{~L}$ \\
\hline & $\mathrm{Cys}\left(\mathrm{SO}_{3} \mathrm{H}\right)^{a, c}$ & $575-576$ & 11.59 & 12.66 & $\mathrm{D}$ \\
\hline & Thr1, Thr2 ${ }^{a}$ & $525-526$ & 27.62 & 33.78 & $\mathrm{~L}$ \\
\hline & $\mathrm{NMeGln}^{a}$ & $567-568$ & 28.64 & 29.58 & $\mathrm{~L}$ \\
\hline & Phe $^{d}$ & $457.5-458.8$ & 43.36 & 49.24 & $\mathrm{D}$ \\
\hline & $\mathrm{Asn}^{d}$ & $524.5-525.0$ & 36.85 & 36.85 & \\
\hline \multirow[t]{2}{*}{ Standard amino acids } & L-allo-Thr & $525-526$ & 28.82 & 31.27 & \\
\hline & L-Asn ${ }^{e}$ & $384.5-385.5$ & $\begin{array}{c}47.80 \\
\text { L-AA-L-DAA }\end{array}$ & $\begin{array}{c}56.20 \\
\text { D-AA-L-DAA }\end{array}$ & \\
\hline 2 & $\mathrm{Asn}^{e}$ & $384.5-385.5$ & 47.73 & & $\mathrm{~L}$ \\
\hline 3 & $\mathrm{Asn}^{e}$ & $384.5-385.5$ & 47.70 & & $\mathrm{~L}$ \\
\hline
\end{tabular}

a The ions of advanced Marfey's reaction products were detected as form of $\left[\mathrm{M}+\mathrm{CF}_{3} \mathrm{COO}\right]^{-}$under negative ion detection mode.

${ }^{\mathrm{b}}$ The ions of advanced Marfey's reaction products were detected as form of $\left[\mathrm{M}+2 \mathrm{CF}_{3} \mathrm{COOH}-\mathrm{H}\right]^{-}$under negative ion detection mode.

${ }^{c}$ The L-AA-D-DLA or D-AA-L-DLA products derived from the amino acids with highly polar residues such as Arg and Cys $\left(\mathrm{SO}_{3} \mathrm{H}\right)$ elute faster than L-AA-L-DLA or D-AA-D-DLA products (Fuji et al., Anal. Chem. 1997, 69, 5146-5151).

${ }^{d}$ The ions of advanced Marfey's reaction products were detected as form of [M-H] under negative ion detection mode.

e The absolute configuration of Asn was determined by Marfey's method based on the comparison of retention time of natural Asn-L-DAA to those of two authentic standards (L-Asn-L-DAA 47.80/ D-Asn-L-DAA 56.20 min on positive ion detection $[\mathrm{M}+\mathrm{H}]^{+}$). 
S6. HR-ESI-Tof-MS spectrum of halicylindramide F (1)
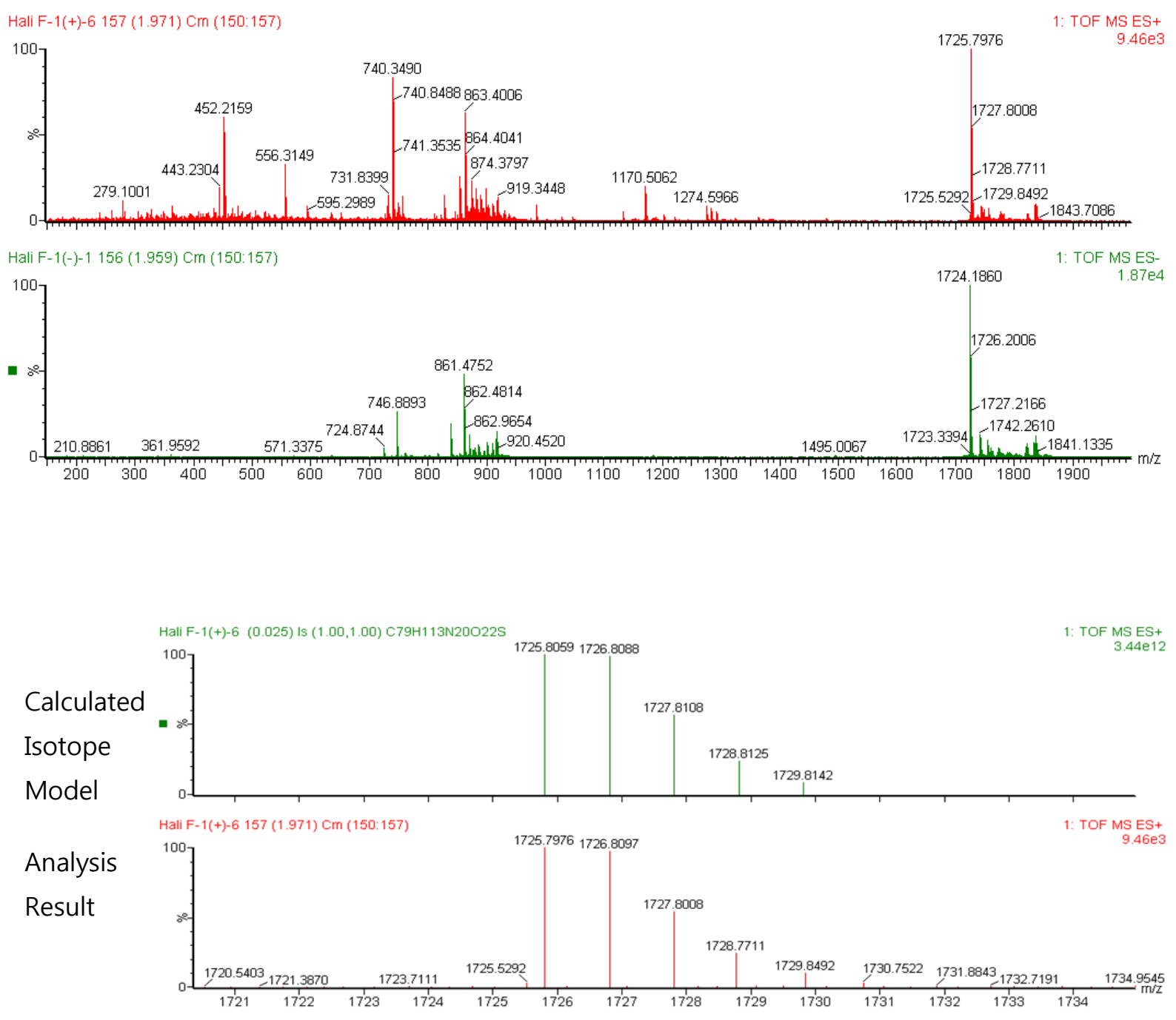

Single Mass Analysis

Tolerance $=5.0$ PPM / DBE: $\min =-1.5, \max =50.0$

Selected filters: None
Monoisotopic Mass, Even Electron lons

12 formula(e) evaluated with 2 results within limits (up to 50 best isotopic matches for each mass)

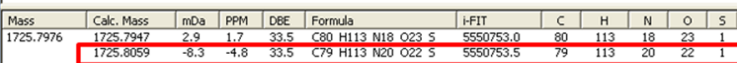

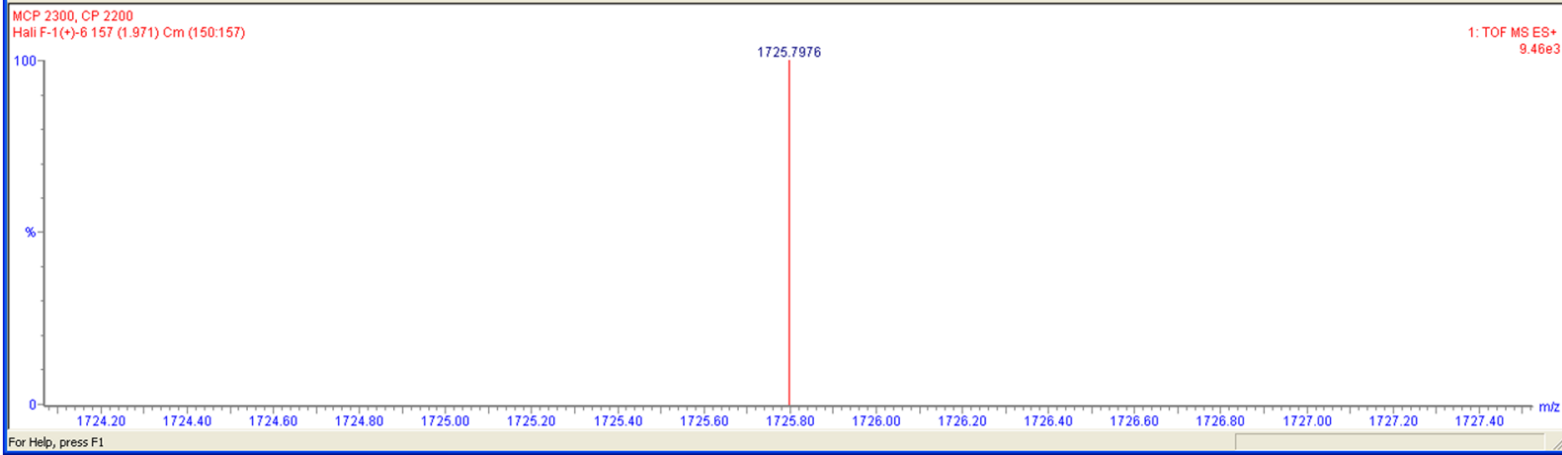


S7. 1H NMR (600 MHz) spectrum of halicylindramide F (1) in DMSO-d6

$: 6$

E
E.
ம0
0
0
0

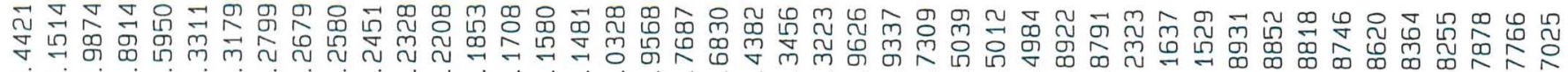
w
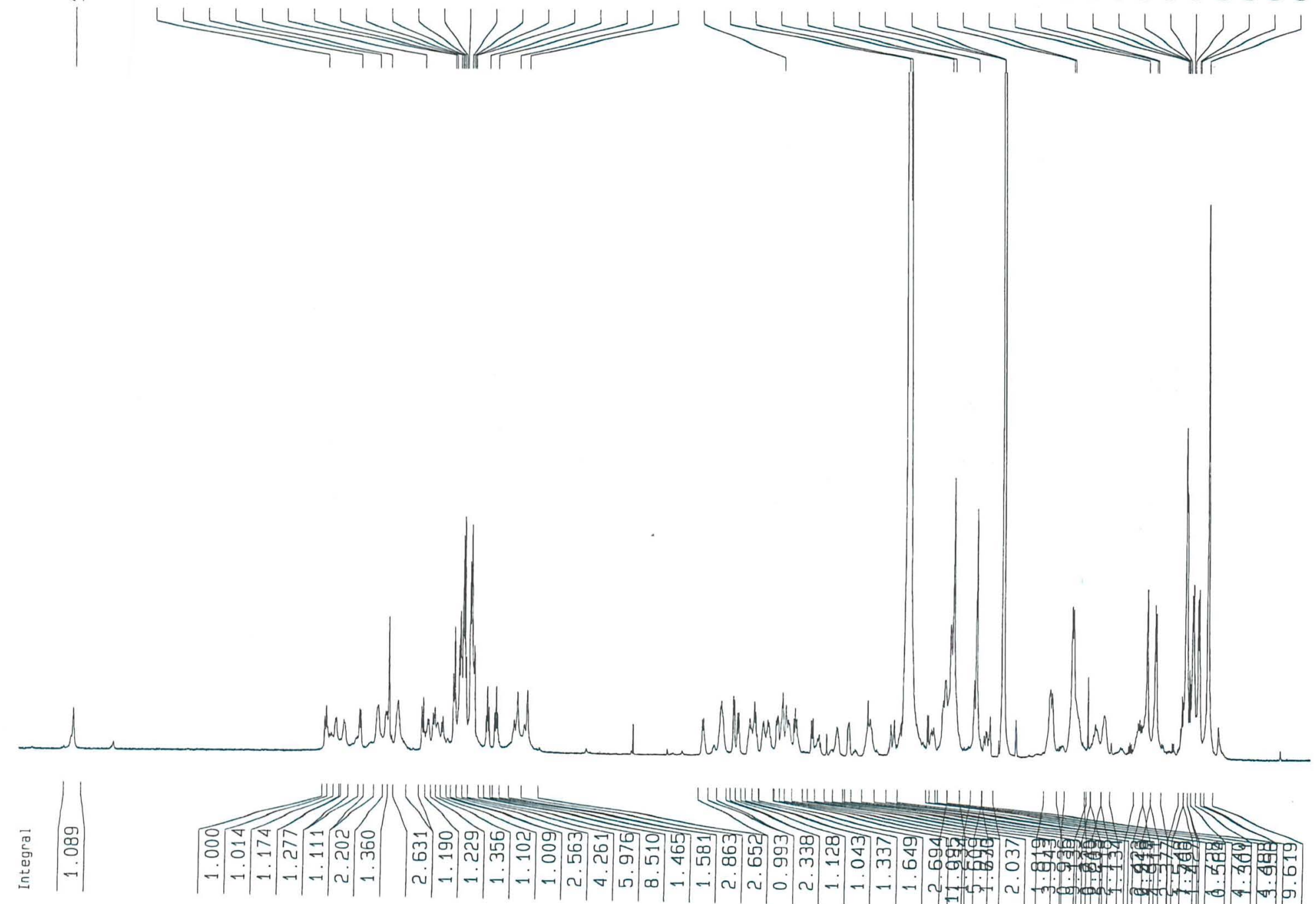

ָ口

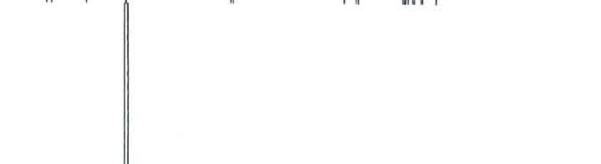

Current

NAME

EXPNO

PAOCNO

107-snu-khj

F2 - Acquisition Parameters

Date_ 20040707

Time 19.29

INSTAUM spect

PAOBHD $5 \mathrm{~mm}$ CPTXI $1 \mathrm{H} /$

$\begin{array}{lr}\text { PULPPOG } & 2930 \\ \text { TD } & 65536\end{array}$

SOLVENT DMSO

SOLV

NS
DS

SWH $\quad 8741.259 \mathrm{~Hz}$

PIDRES $\quad 0.133381 \mathrm{~Hz}$

AQ 3.7487664 se

$\begin{array}{ll}\text { RG } & 32 \\ \text { DW } & 57.200 \text { usec }\end{array}$

DE 6.00 usec

TE $297.9 \mathrm{~K}$

$01 \quad 1.00000000 \mathrm{sec}$

MCREST $\quad 0.00000000 \mathrm{sec}$

MCWAK $\quad 0.01500000 \mathrm{sec}$

$====s==$ CHANNEL

PL1 10.30 usec

$5.00 \mathrm{~dB}$

F2 - Processing parameters

$\begin{array}{lc}\text { SI } & 32768 \\ \text { SF } & 600.1300063 \mathrm{MHz}\end{array}$

WDW

SSB

$\angle B-0$

GB

10 NMR plot parameters

CX $\quad 20.00 \mathrm{~cm}$

CY $\quad 1000.00 \mathrm{~cm}$

F1P $\quad 11.126 \mathrm{ppm}$

F1 $\quad 6677.05 \mathrm{~Hz}$

F2P $\quad-0.198 \mathrm{ppm}$

$\begin{array}{ll}F 2 & -119.04 \mathrm{~Hz}\end{array}$

$\begin{array}{ll}\text { PPMCM } & 0.56622 \mathrm{ppm} / \mathrm{cm}\end{array}$ 
S8. 13C NMR (150 MHz) spectrum of halicylindramide F (1) in DMSO-d6

/C13

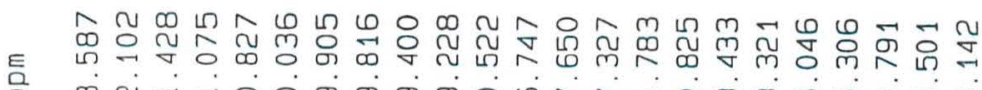

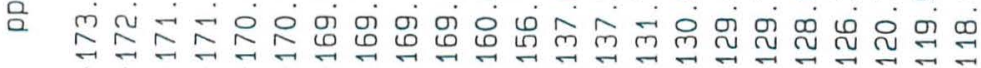

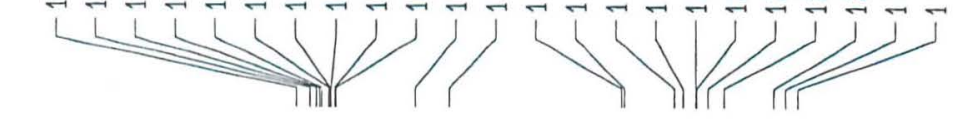

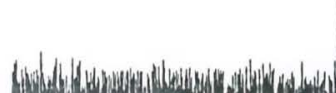

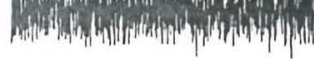

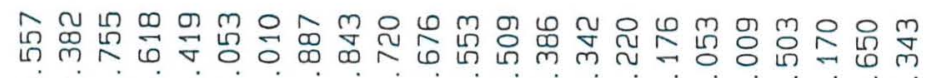

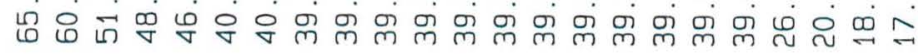

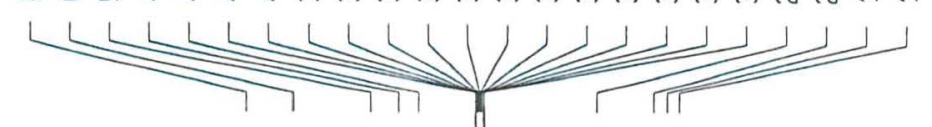

Current Data Parameters

NAME

PROCNO

F2 - Acquisition Parameter

Date_ 20040816

Time

INSTRUM

$5 \mathrm{~mm}$ BBI $1 \mathrm{H} / 2 \mathrm{H}$

$\begin{array}{lr}\text { TO } & \text { ZgdC } \\ \text { TD } & 65536\end{array}$

SOLVENT DMSO

$\begin{array}{lr}\text { NS } & 64657 \\ \text { DS } & \end{array}$

DSH $\quad 30303.031 \mathrm{~Hz}$

FIDRES $\quad 0.462388 \mathrm{~Hz}$

$A Q \quad 1.0814105 \mathrm{sec}$

DW $\quad \begin{array}{r}32768 \\ 16.500 \text { usec }\end{array}$

DE 6.00 use 6.00 use
$300.0 \mathrm{~K}$

2. $00000000 \mathrm{sec}$

d11

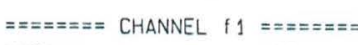

NUC1 $13 \mathrm{C}$

PL1 $\quad 0.00 \mathrm{~dB}$

SF01 $\quad 125.7709436 \mathrm{MHz}$

$== \pm=z==$ CHANNEL f2 $=== \pm==0$

$\begin{array}{lr}\text { CPDPRG2 } & \text { waltz16 } \\ \text { NUC2 } & 1 \mathrm{H}\end{array}$

$\begin{array}{lr}\text { NUC2 } & 1 \mathrm{H} \\ \text { PCPD2 } & 100.00 \text { usec }\end{array}$

$\begin{array}{lr}\text { PL2 } & 0.00 \mathrm{~dB}\end{array}$

PL12 $\quad 500.1320005 \mathrm{~dB}$

F2 - Processing parameters

$\begin{array}{lr}\text { SI } & \text { P2768 } \\ \text { SF } & 125.7578452 \mathrm{MHZ}\end{array}$

WDW $\quad$ EM

$\begin{array}{ll}\text { SSB } & 0 \\ \text { LB } & \end{array}$

GB

10 NMR plot parameters

$\begin{array}{lr}\text { CX } & 20.00 \mathrm{~cm} \\ \mathrm{CY} & 200.00 \mathrm{~cm}\end{array}$

$210.000 \mathrm{ppm}$
$\mathrm{FI}$

$\begin{array}{ll}F_{1} & 26409.15 \mathrm{~Hz} \\ \text { F2P } & -10.000\end{array}$

F2 $\quad-1257.58 \mathrm{~Hz}$

$11.00000 \mathrm{ppm} / \mathrm{c}$ 
S9. HMQC (600 MHz) spectrum of halicylindramide $\mathrm{F}$ (1) in DMSO-d6

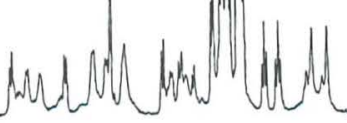

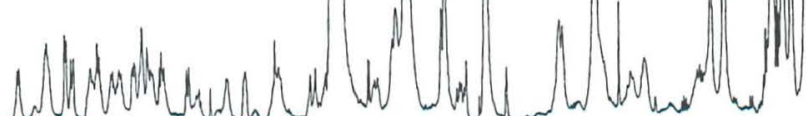
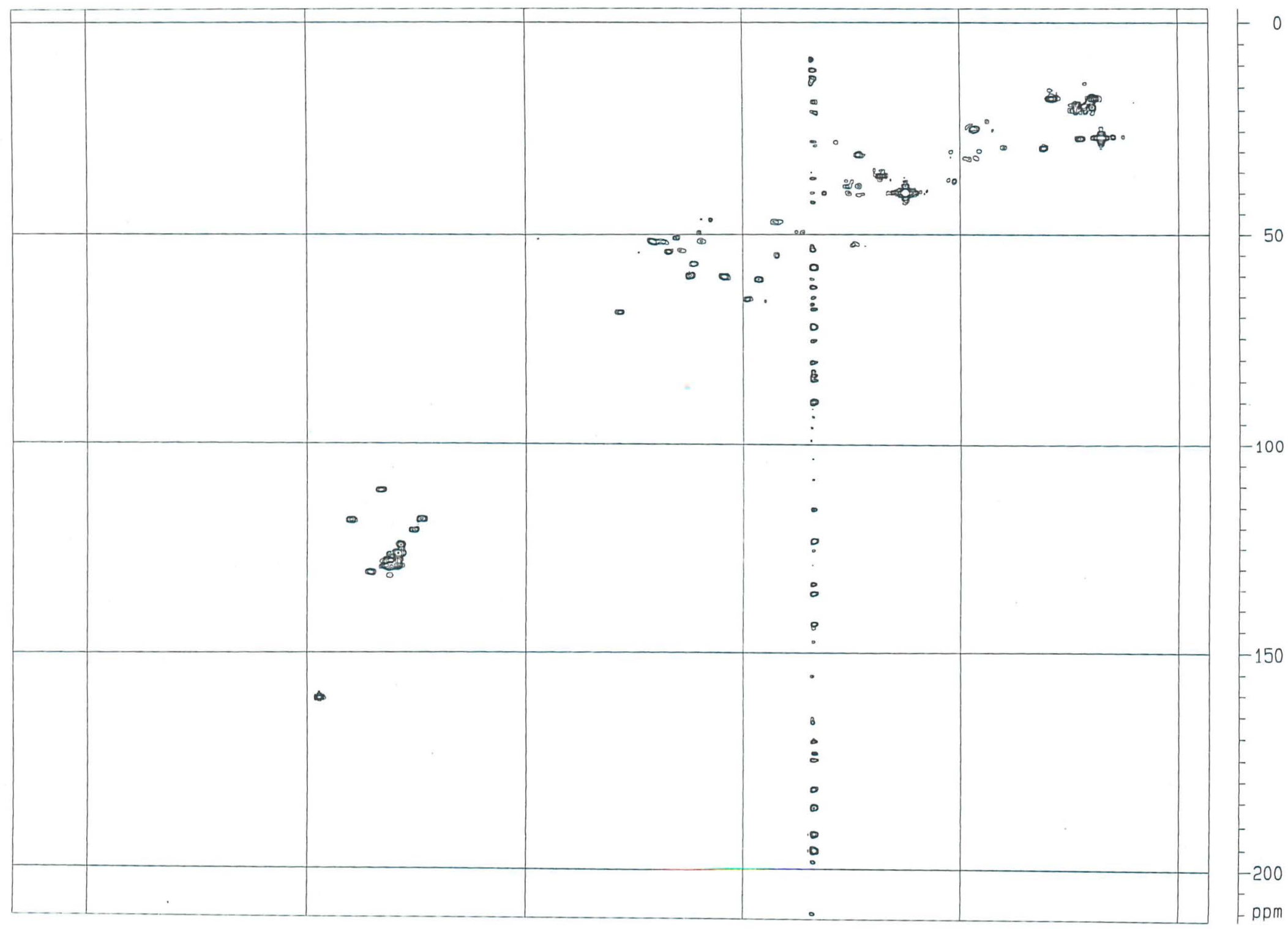

ppm 10

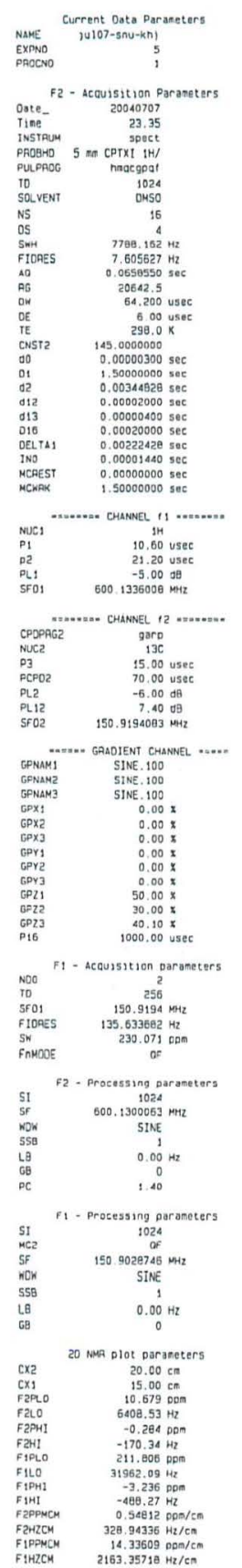




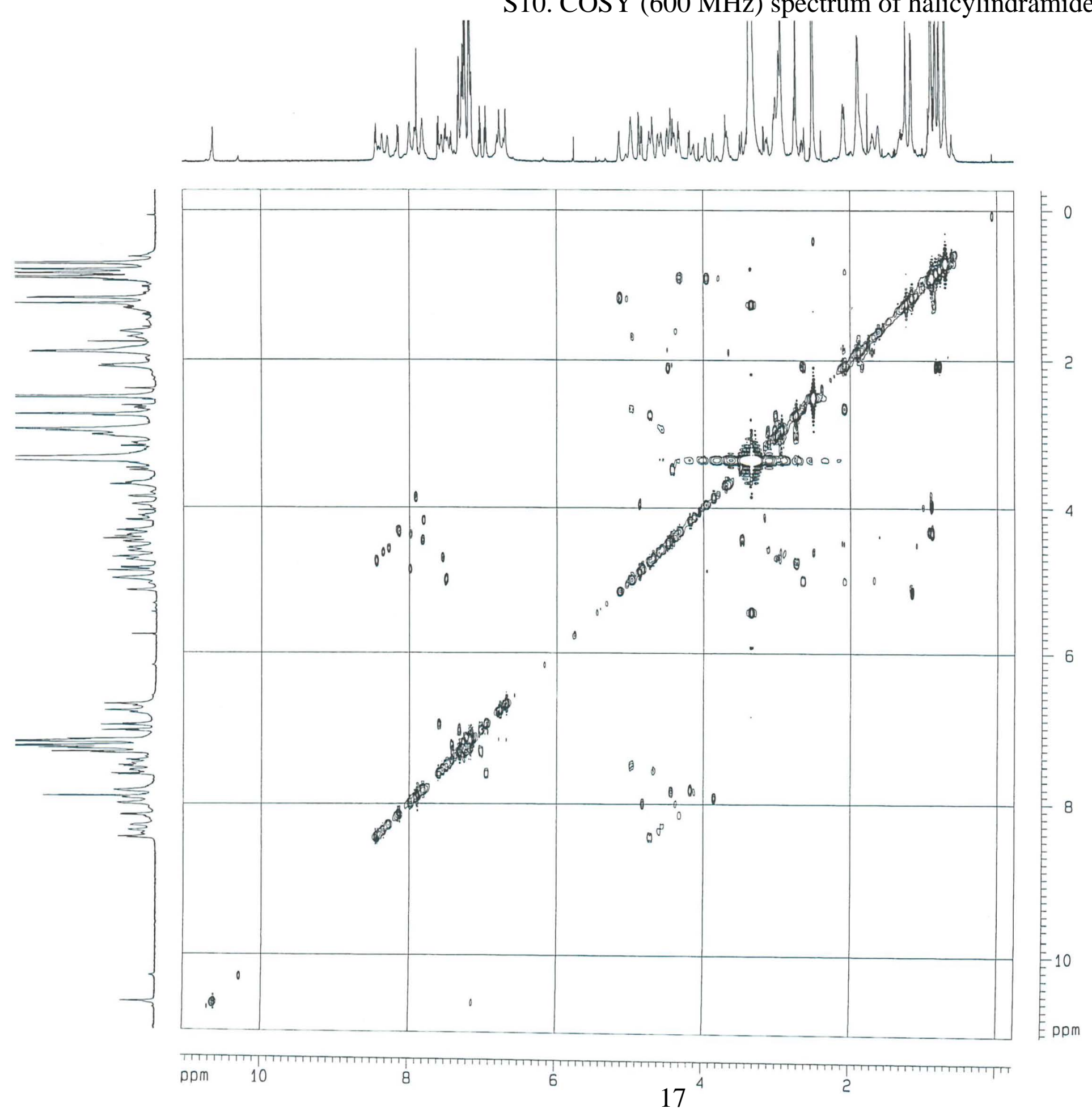

/COSY

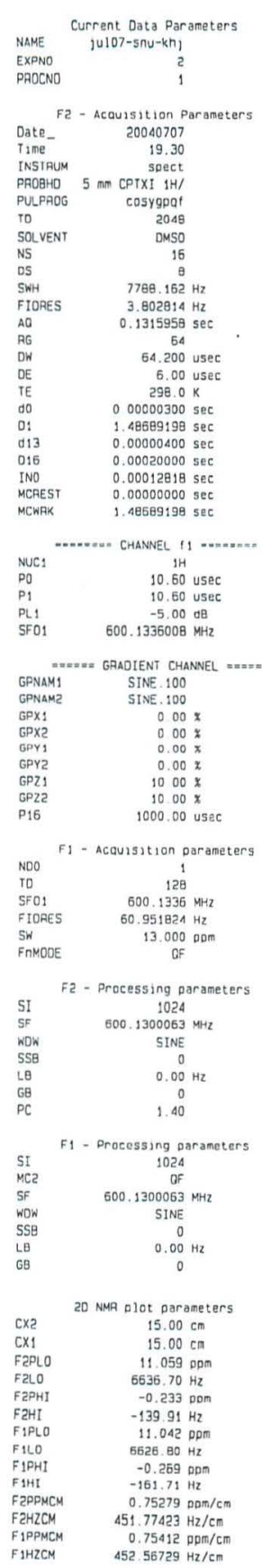


S12. Expanded COSY (600 MHz) spectrum of halicylindramide F (1) in DMSO-d6

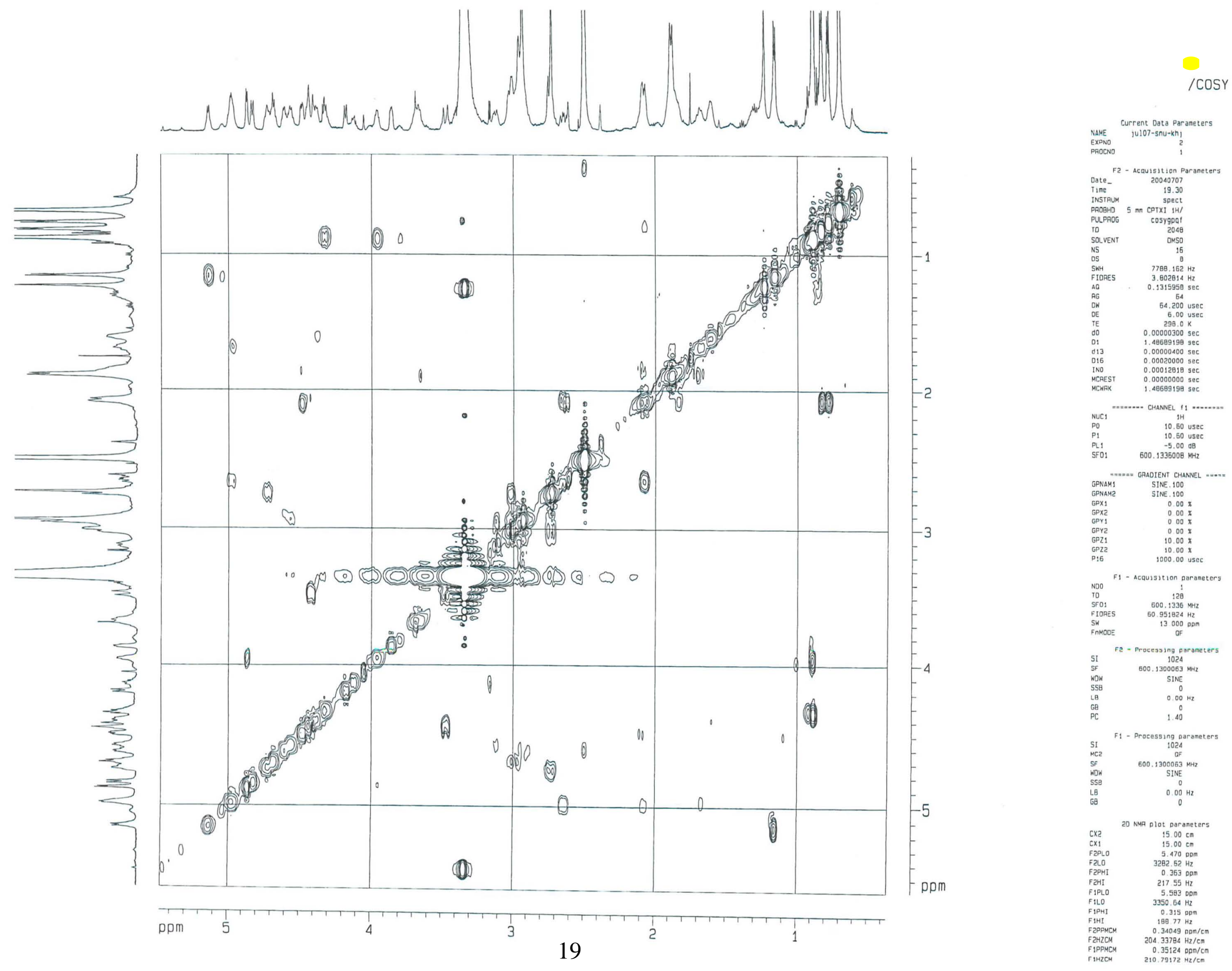


S13. TOCSY (600 MHz) spectrum of halicylindramide F (1) in DMSO-d6

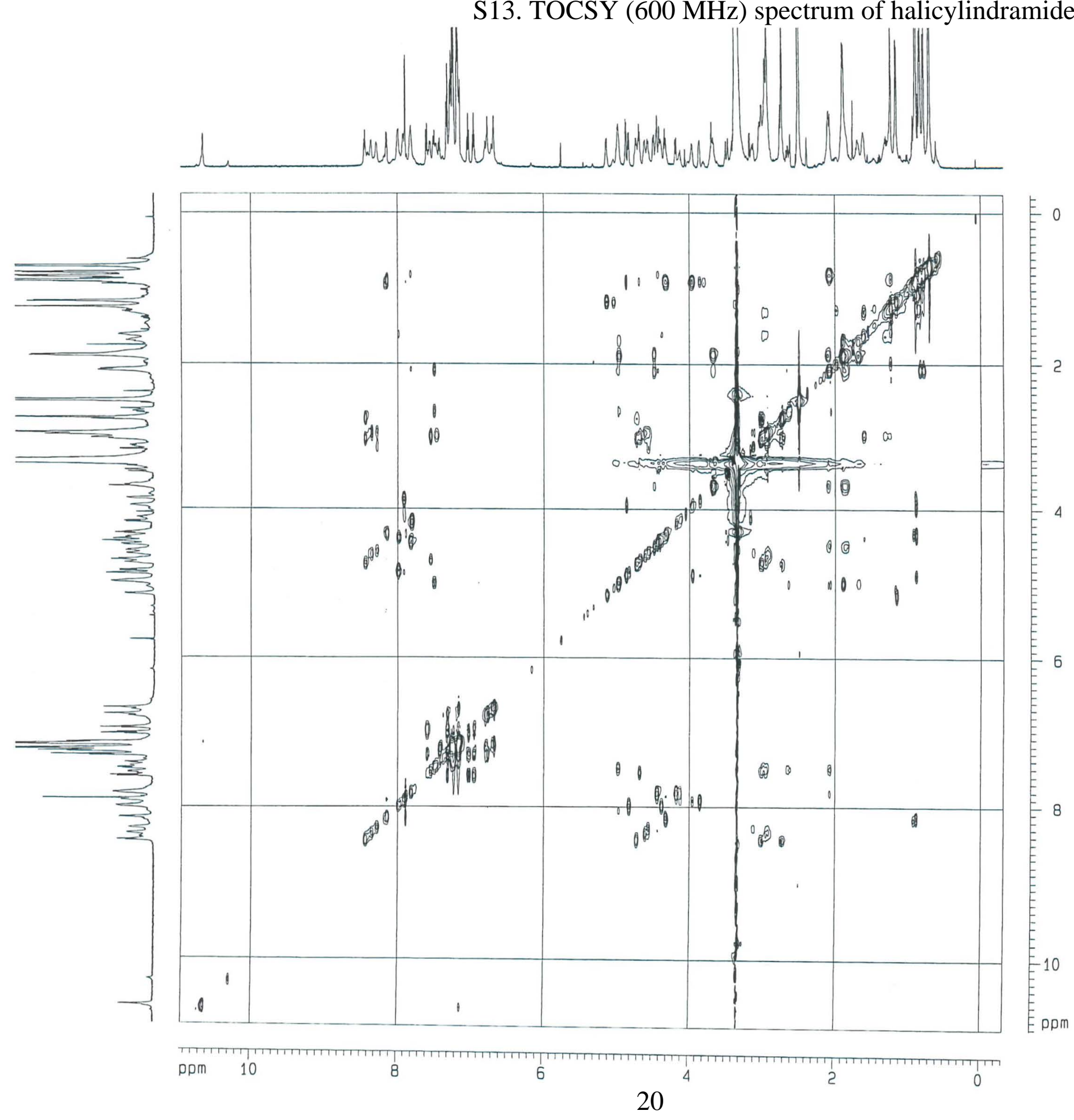

6/TOCSY

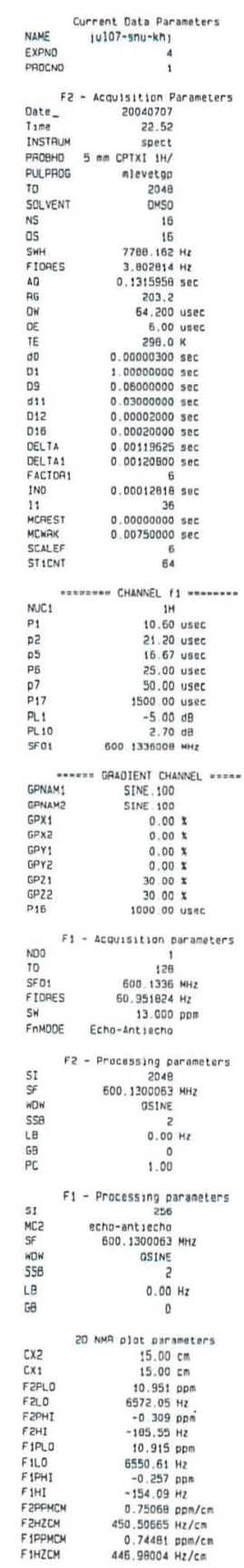


S14. Expanded TOCSY (600 MHz) spectrum of halicylindramide F (1) in DMSO-d6

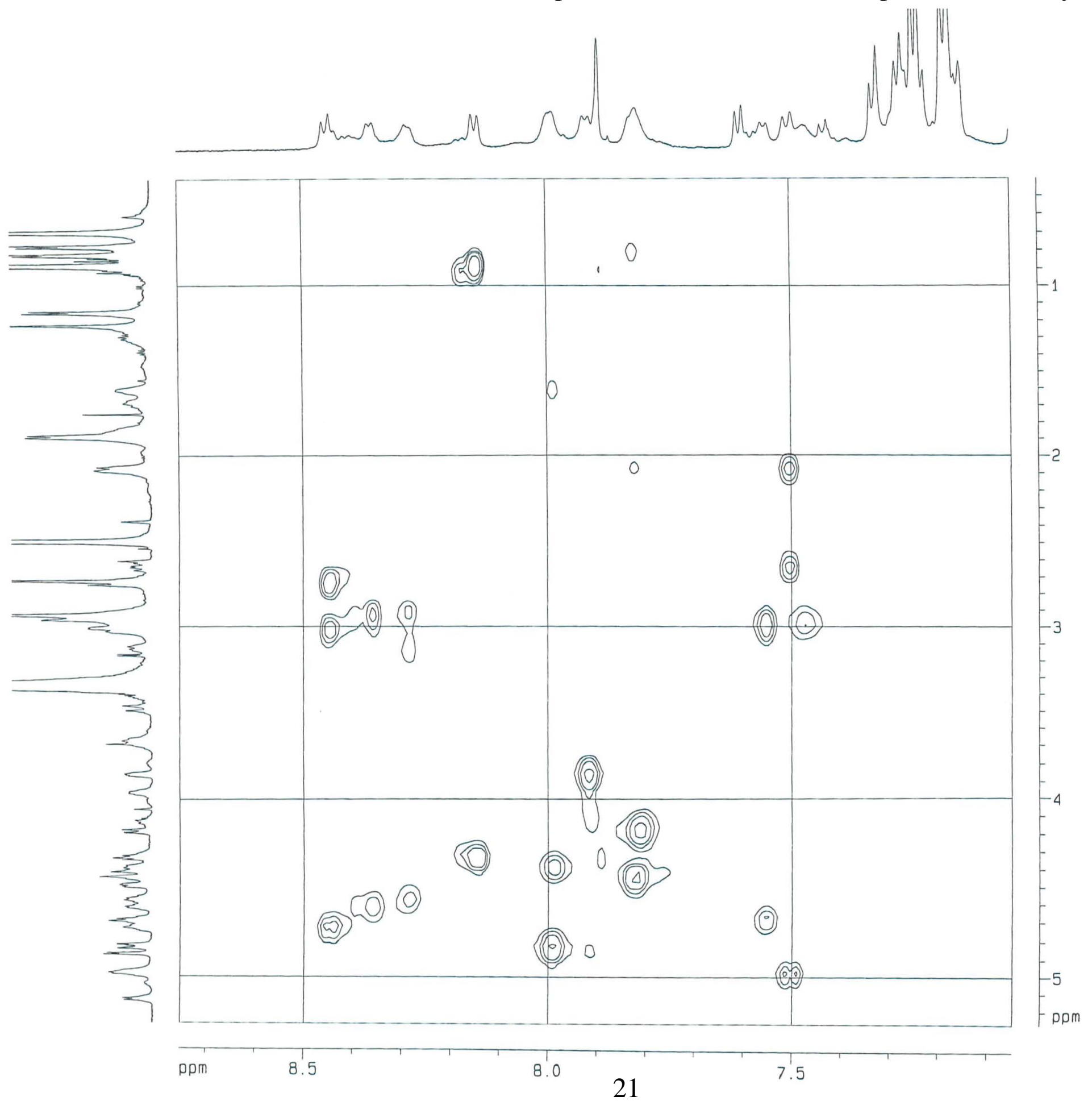

6/TOCSY

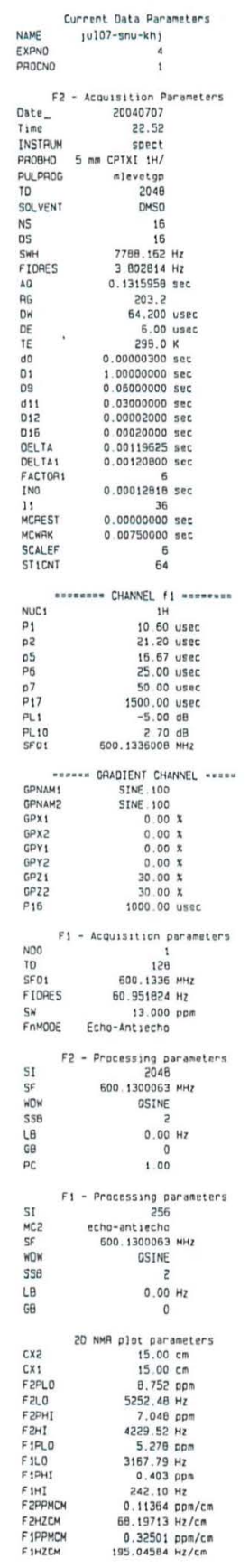


S15. Expanded TOCSY (600 MHz) spectrum of halicylindramide F (1) in DMSO-d6

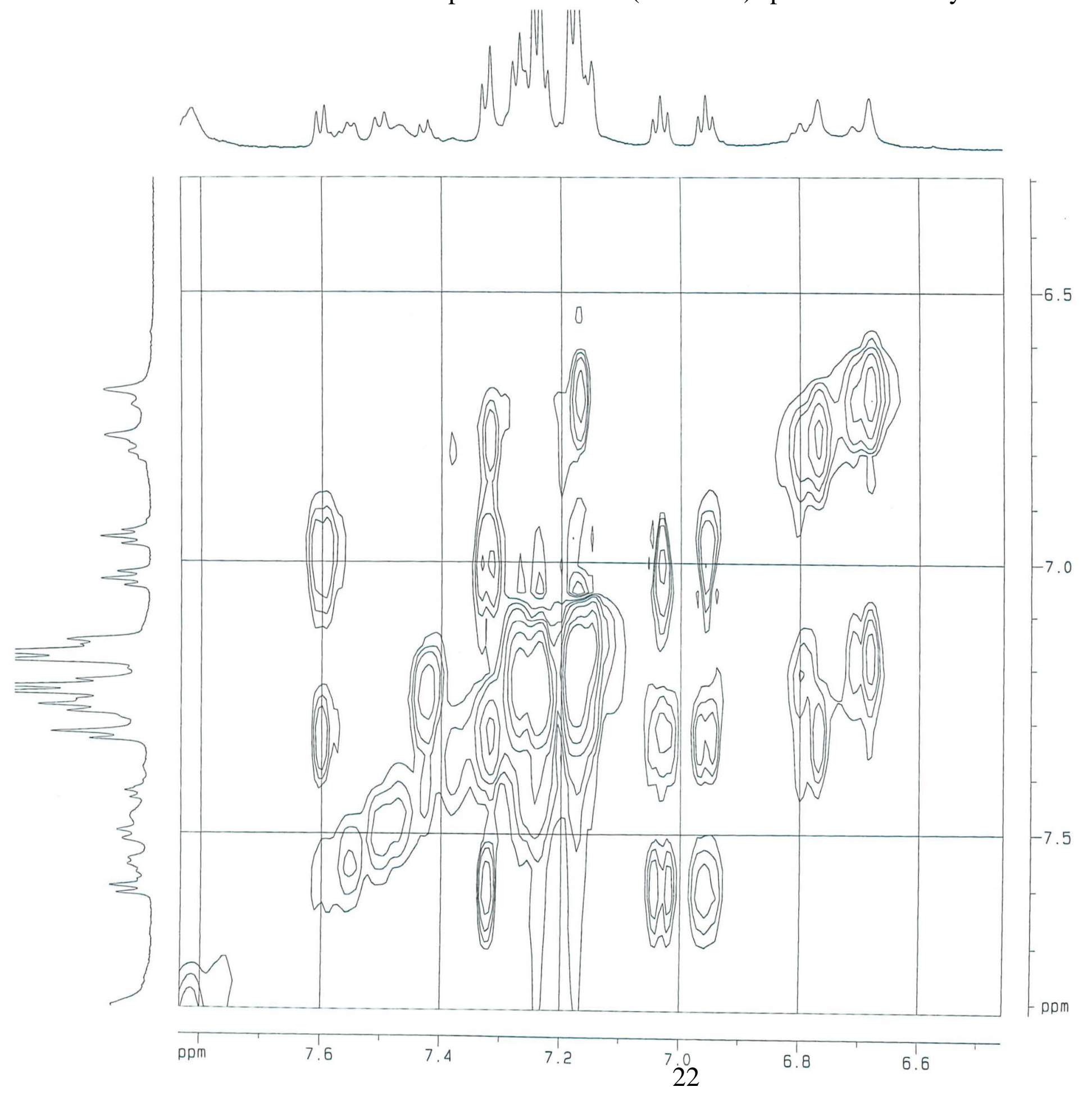

6/TOCSY

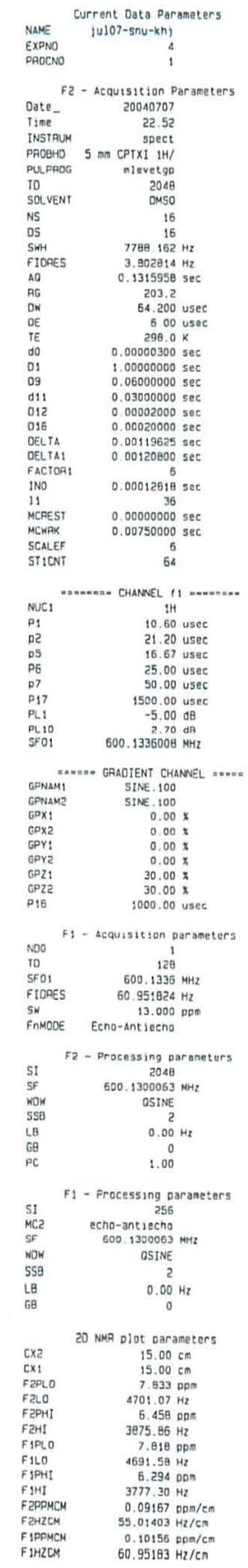


S16. Expanded TOCSY (600 MHz) spectrum of halicylindramide F (1) in DMSO-d6

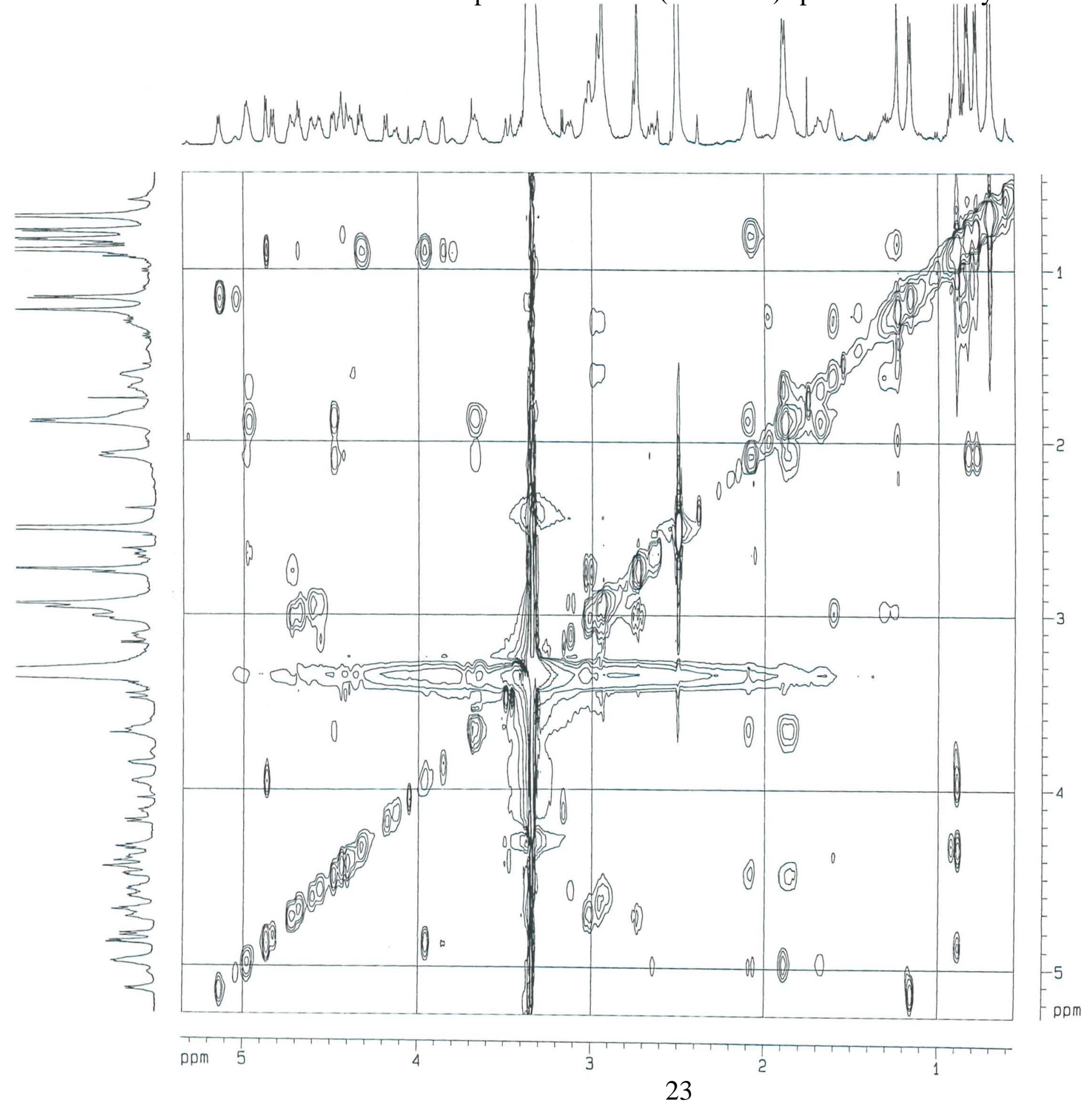

6/TOCSY

Nave Current Data paraneters

XXPEN
PROCNO

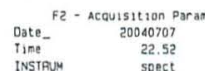

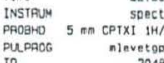

Mvant an

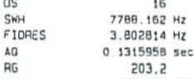

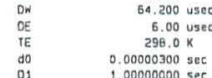

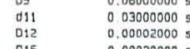

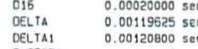

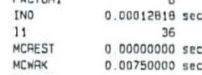

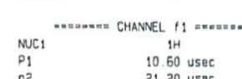

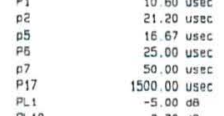

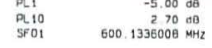

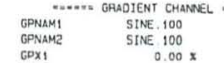

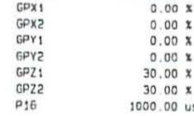

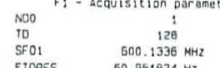

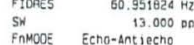

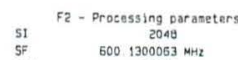

tor

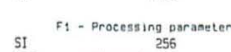

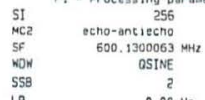

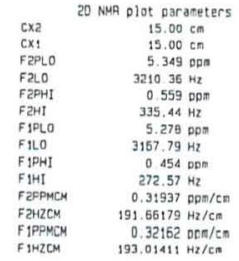


S17. NOESY (600 MHz) spectrum of halicylindramide F (1) in DMSO-d6

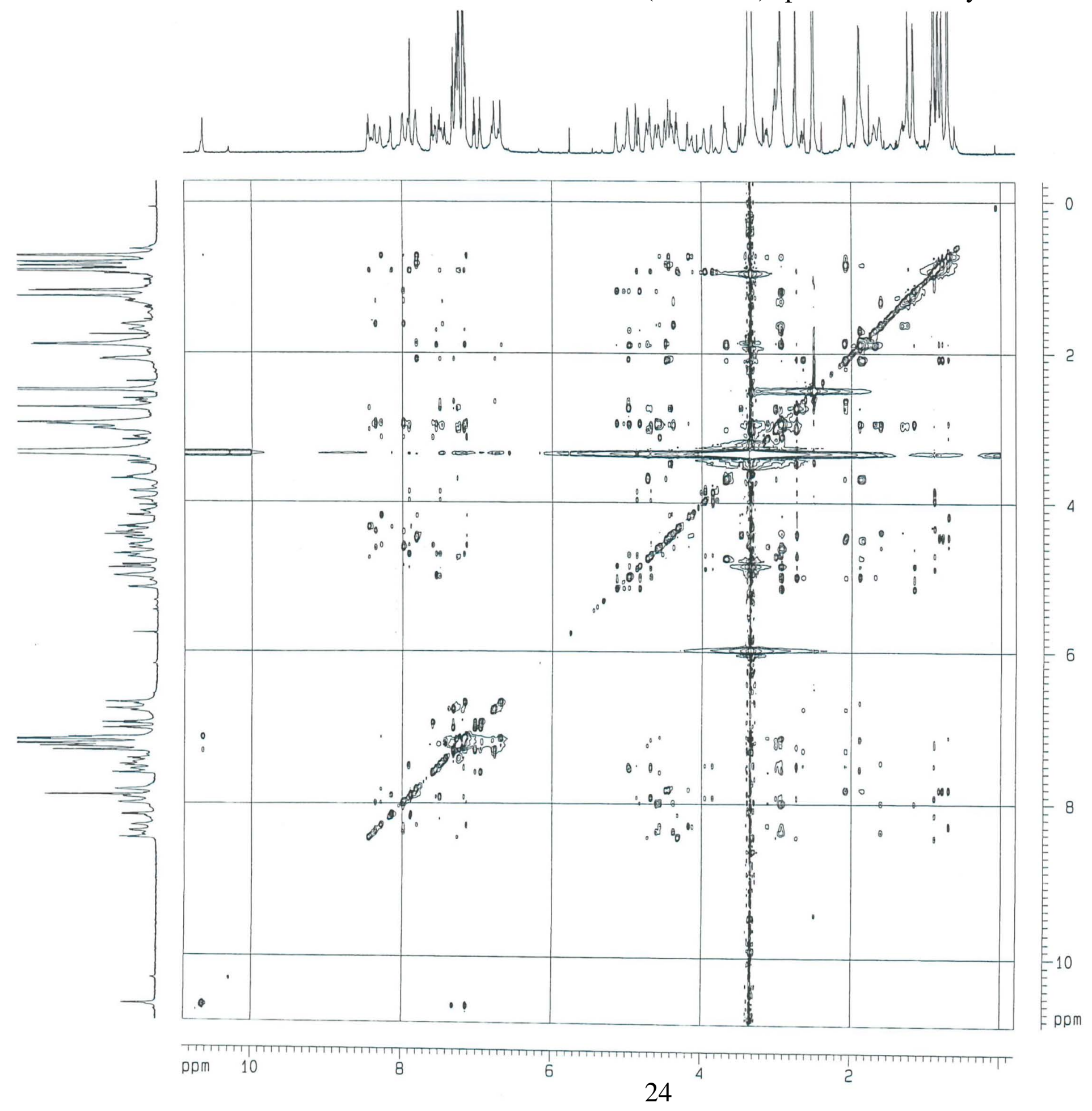

6/NOESY

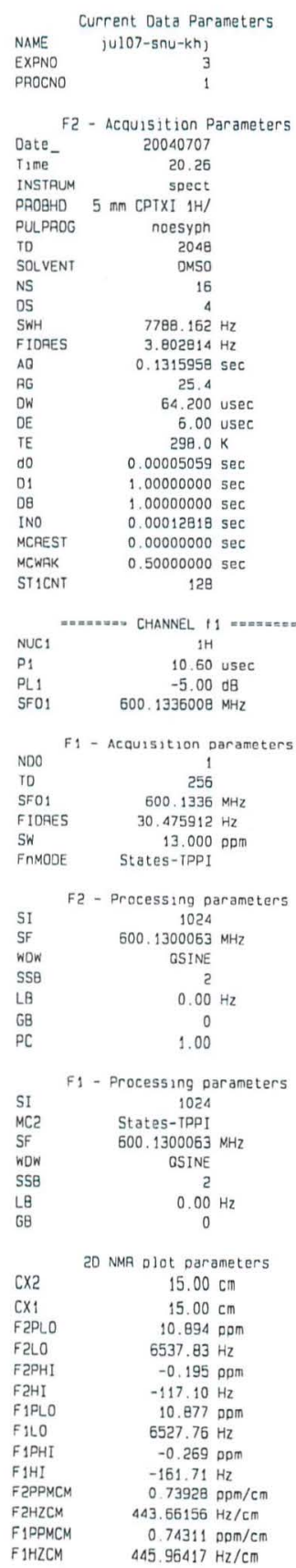


S18. Expanded NOESY (600 MHz) spectrum of halicylindramide F (1) in DMSO-d6

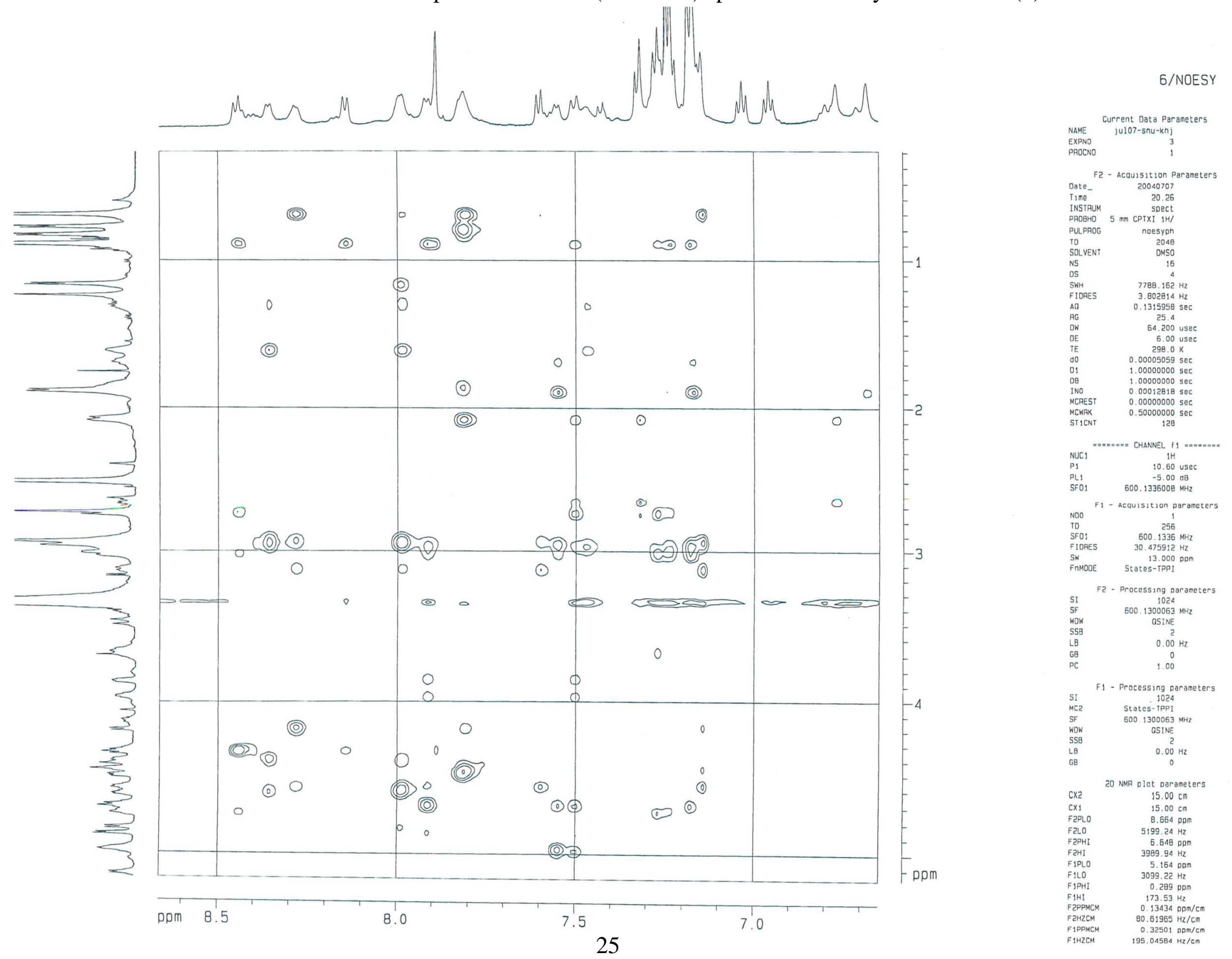


S19. Expanded NOESY (600 MHz) spectrum of halicylindramide F (1) in DMSO-d6

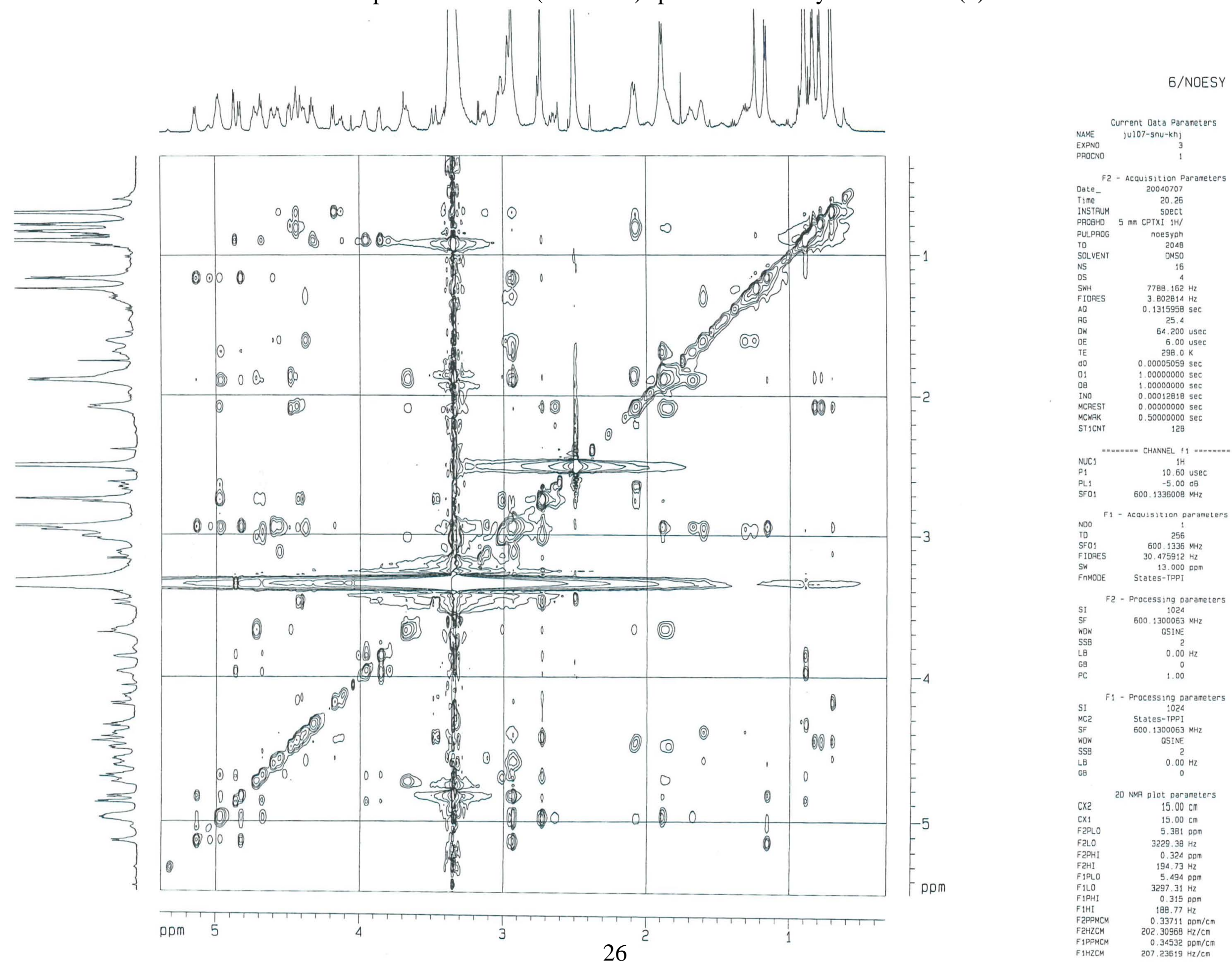


S20. Expanded NOESY (600 MHz) spectrum of halicylindramide F (1) in DMSO-d6

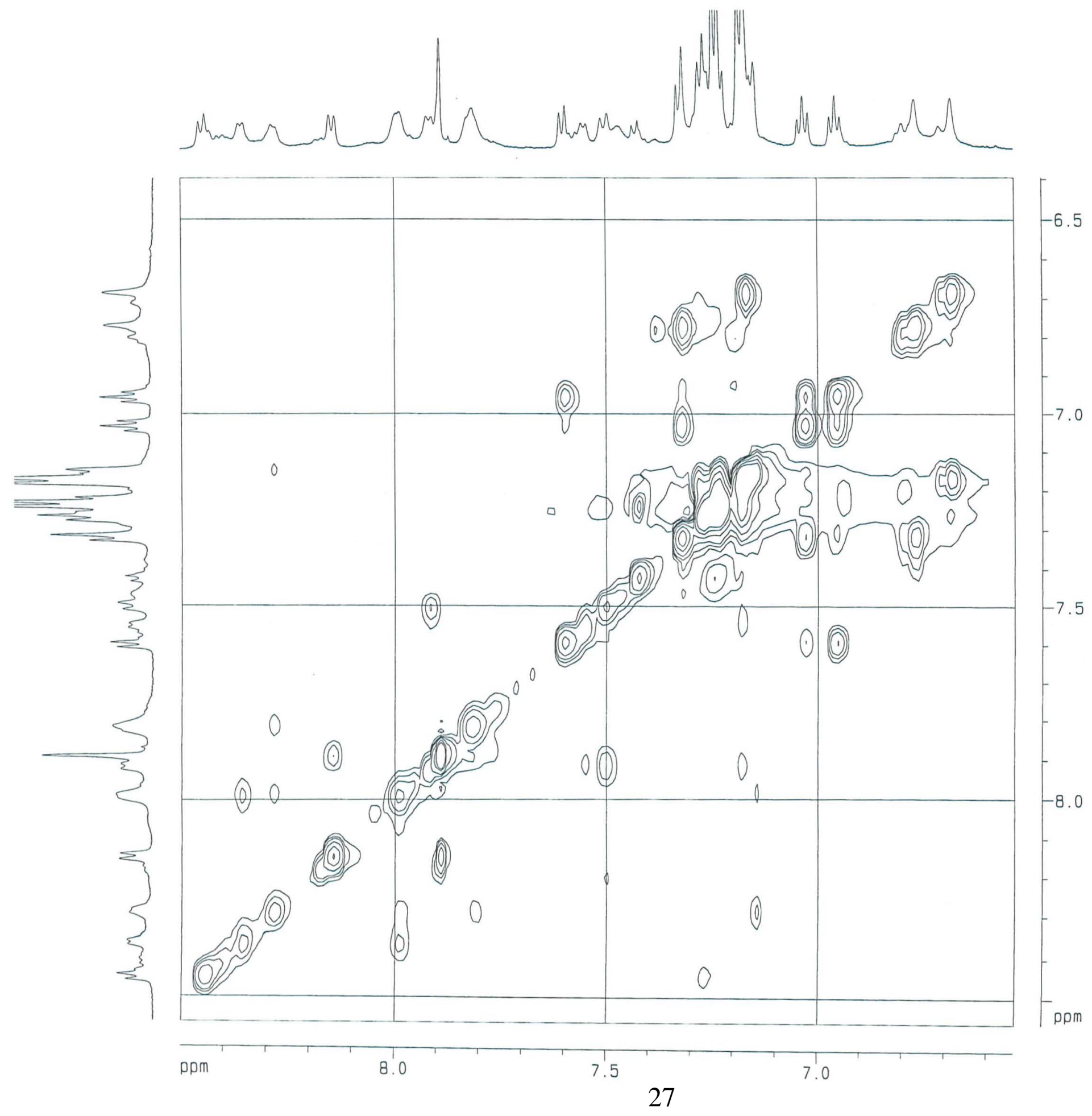

6/NOESY

Current Data Parameters

EXPNO

F2 - Acquisition Parameters

Date - 2000007

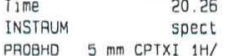

PULPROG
TD noesyph
2048

$\begin{array}{lrl}\text { SOLVEN } & \text { DMSO } \\ \text { NS } & 16\end{array}$

$\begin{array}{ll}\text { SWH } & 47 \\ \text { SWH } & 7788.162 \mathrm{HZ}\end{array}$

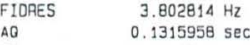

$\begin{array}{lr}\text { AG } & 25.4 \\ \text { OW } & 54.200 \text { usec } \\ \text { OE } & 5.00 \text { use }\end{array}$

$\begin{array}{cc}\text { DE } & 6.00 \text { us } \\ \text { TE } & 298.0 \mathrm{~K} \\ \text { OD } & 0.00005059 \mathrm{sec} \\ 0 & 100000000 \mathrm{sec}\end{array}$

$08 \quad 100000000 \mathrm{se}$

MCAEST $\quad 0.00000000 \mathrm{sec}$

MTICNT 0.50000000

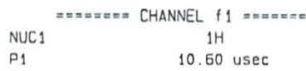

$\begin{array}{lr}\text { P1 } & 10.60 \mathrm{usec} \\ \text { PL. } & -5.00 \mathrm{de} \\ \text { SE } & 500.133500 \mathrm{NHz}\end{array}$

NDO F1 - Acquisition parameters

$\begin{array}{lr}\text { NDO } & 256 \\ \text { TD } & 600.1336 \\ \text { SFO1 } & \text { MHz }\end{array}$

$\begin{array}{ll}\text { SFO1 } & 600.1335 \mathrm{MHz} \\ \text { FIOAES } & 30.475912 \mathrm{~Hz}\end{array}$

13.000
FNMODE DOM

F2 - Processing parameters

$\begin{array}{ll}\text { SI } & 1024 \\ \text { SF } & 600.1300053 \mathrm{MHZ} \\ \text { WOW } & \text { OSINE }\end{array}$

$\begin{array}{ll}\text { SSB } & \text { L } \\ \text { LB } & 0.00 \mathrm{H}\end{array}$

$\begin{array}{ll}68 & 0.00 \\ P C & 1.00\end{array}$

F1 - Processing parameters

SI

600. 1300063 MHZ

1300063 MHZ
OSINE
5
$0.00 \mathrm{HZ}$

20 NNR plot parameters$$
15.00 \mathrm{~cm}
$$

$15.00 \mathrm{~cm}$

8. $499 \mathrm{ppm}$
$5100.36 \mathrm{~Hz}$

$\begin{aligned} 6.534 & \text { pp } \\ 3921.49 \mathrm{~Hz} & \end{aligned}$

$8.567 \mathrm{pom}$

$5141.30 \mathrm{~Hz}$
6.395

$6.396 \mathrm{dpm}$
$3838.26 \mathrm{~Hz}$

$0.13096 \mathrm{ppm} / \mathrm{cm}$

$78.5914 \mathrm{~Hz} / \mathrm{cm}$
$0.14473 \mathrm{ppm} / \mathrm{cm}$ 
S21. HMBC (600 MHz) spectrum of halicylindramide F (1) in DMSO-d6

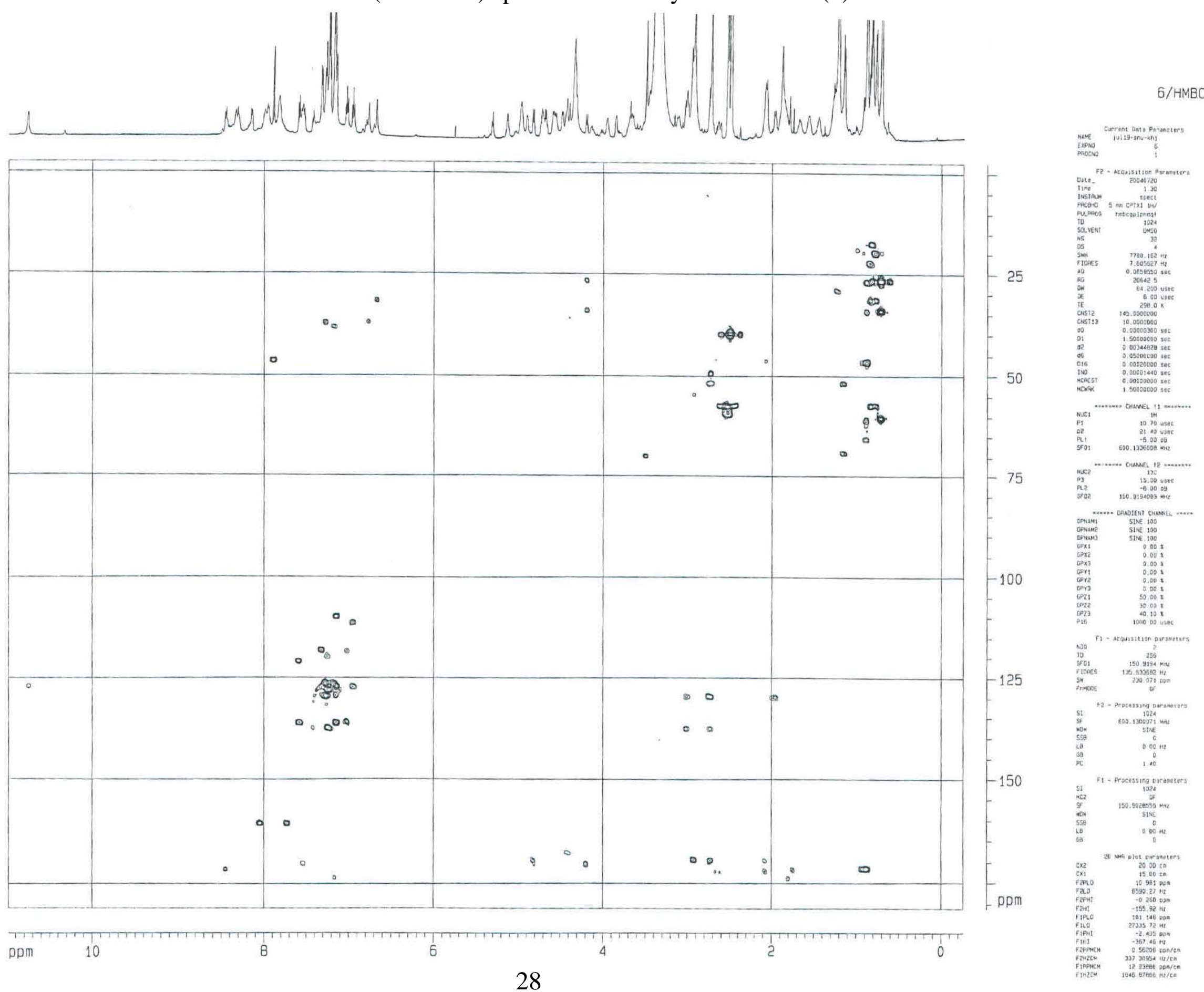



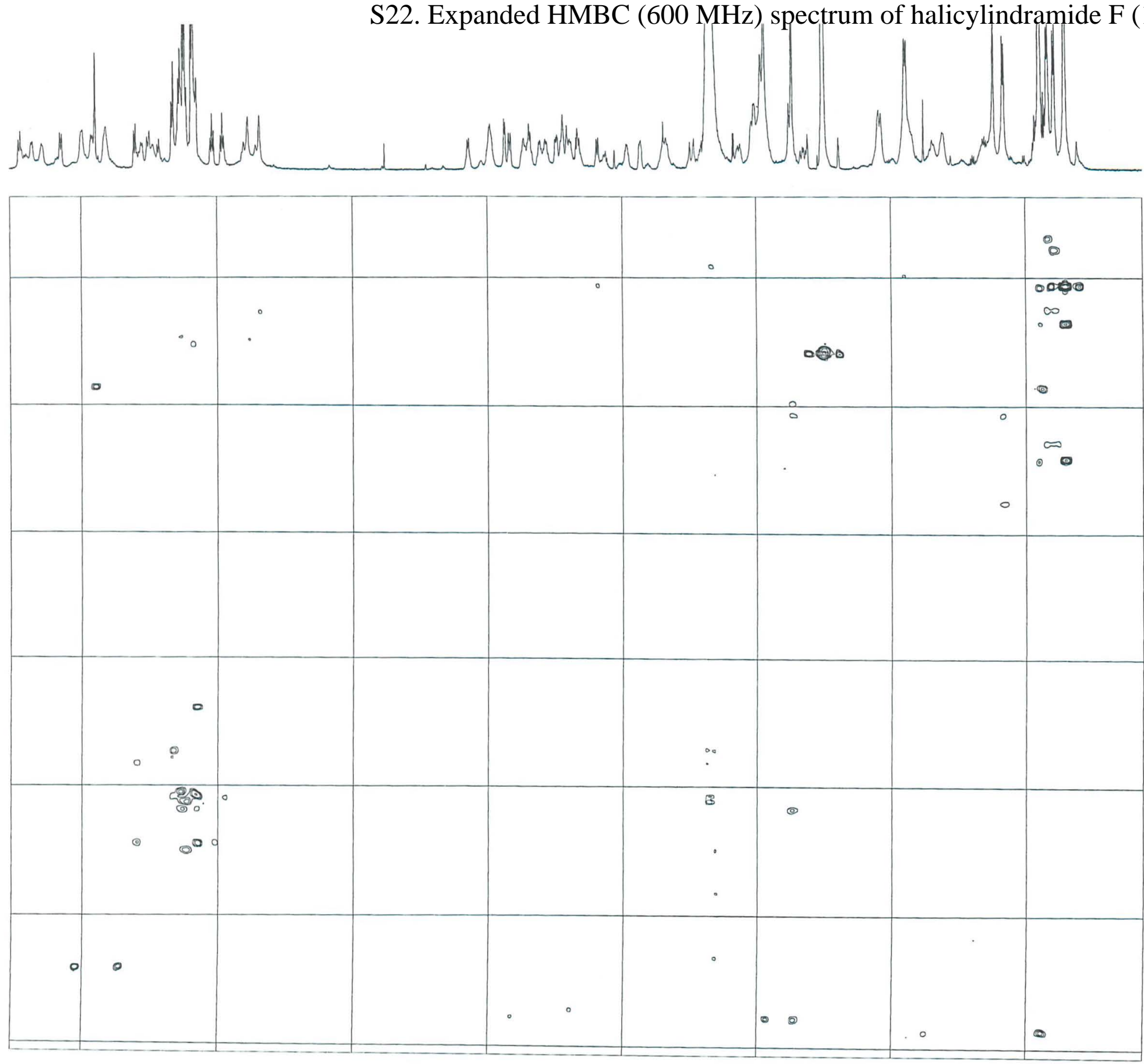
S23. Expanded HMBC (600 MHz) spectrum of halicylindramide F (1) in DMSO-d6

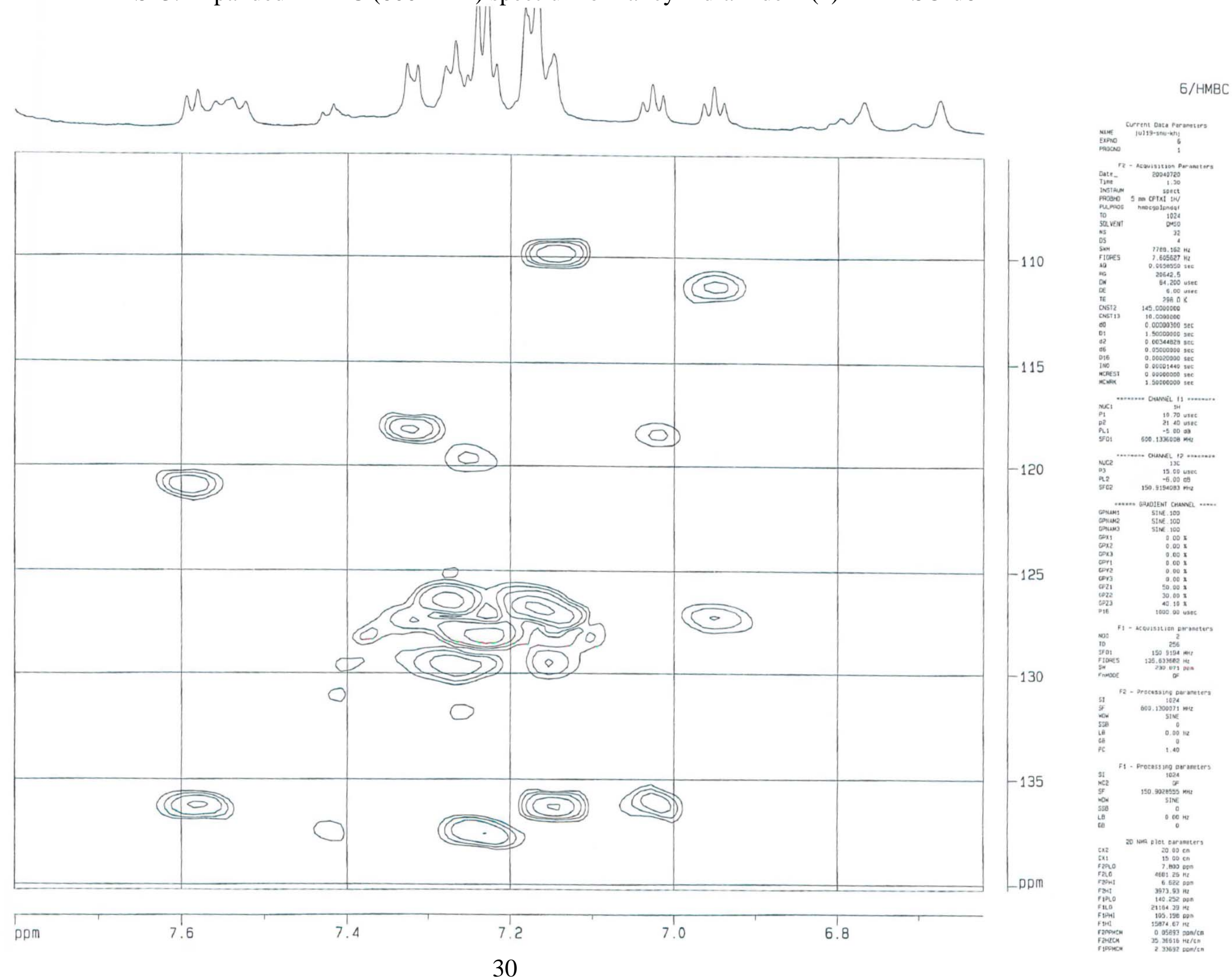


S24. Expanded HMBC (600 MHz) spectrum of halicylindramide F (1) in DMSO-d6

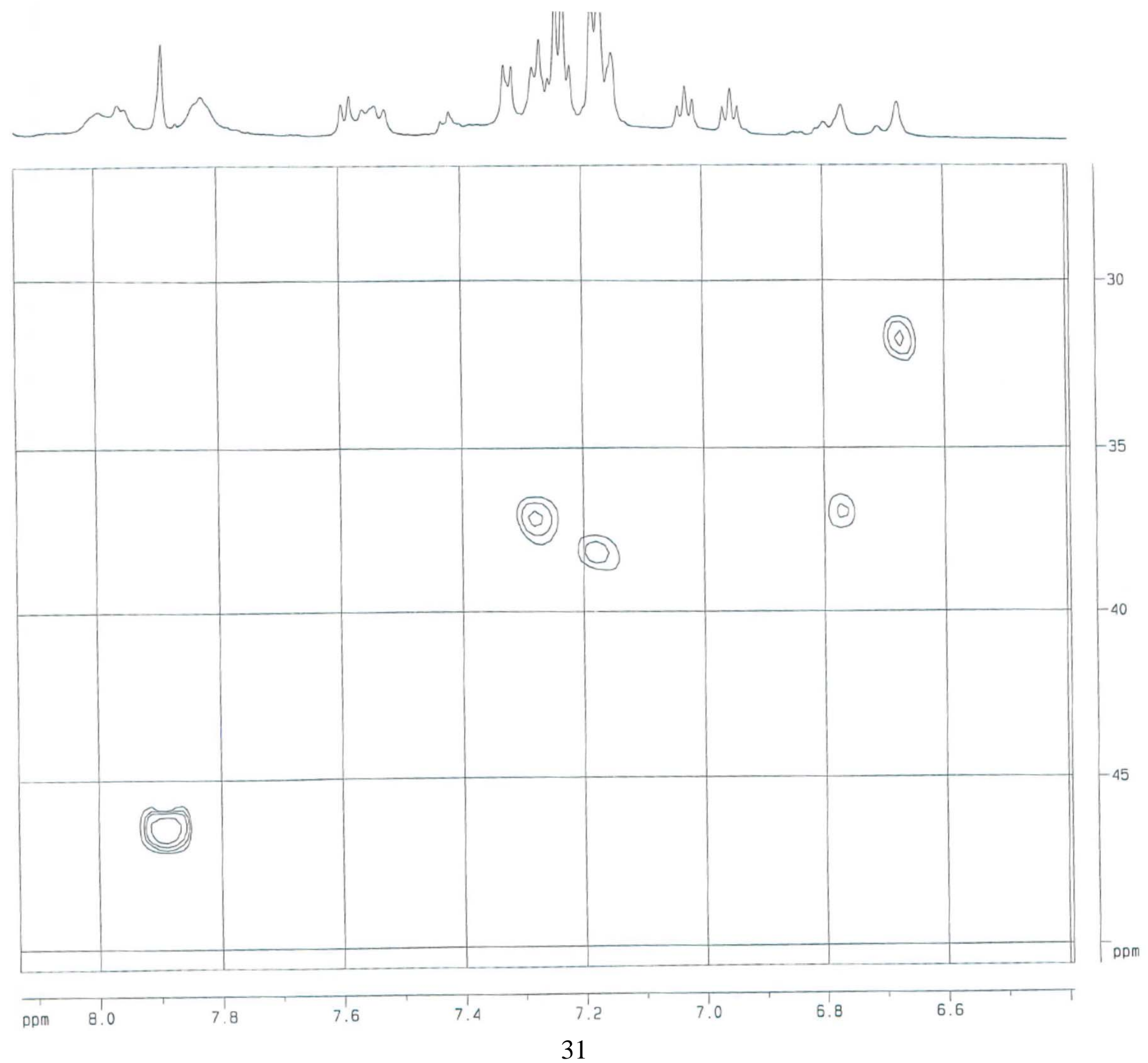

6/HMBC

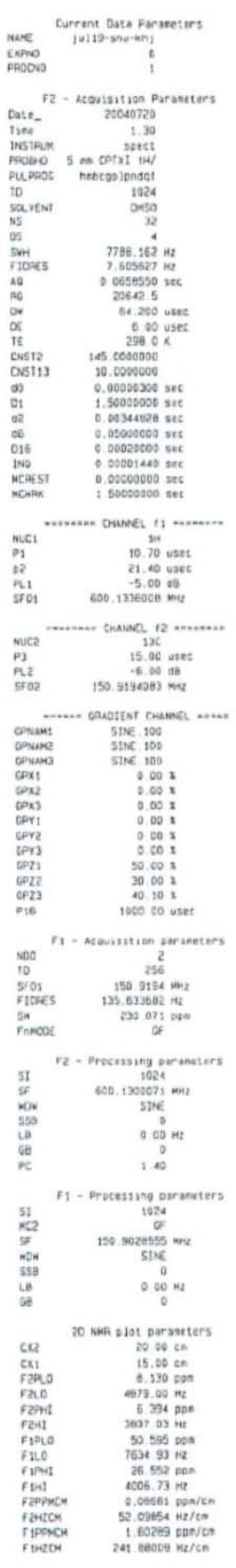


S25. Expanded HMBC (600 MHz) spectrum of halicylindramide F (1) in DMSO-d6
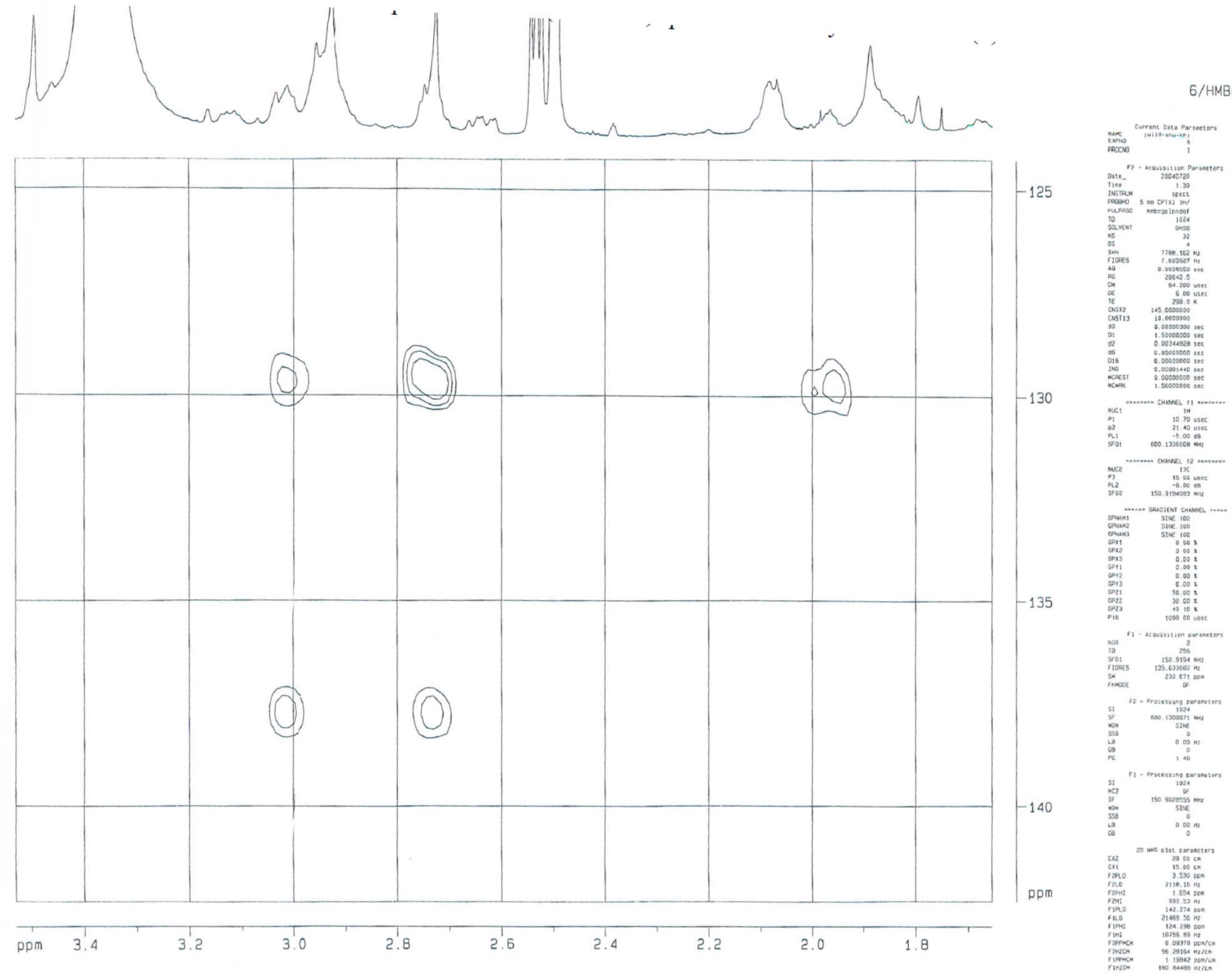
S26. Expanded HMBC (600 MHz) spectrum of halicylindramide F (1) in DMSO-d6
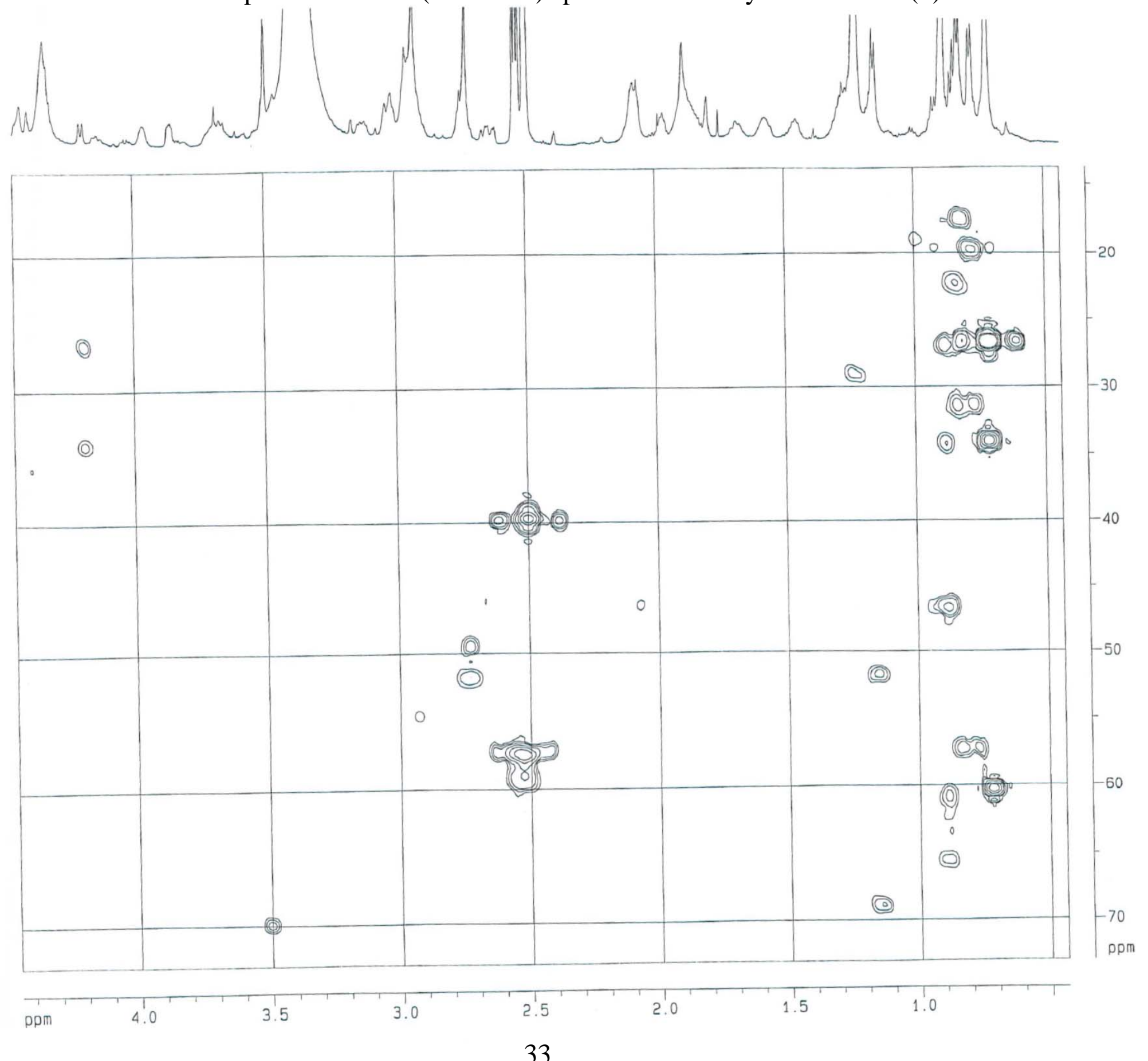

$6 /$ HMBC

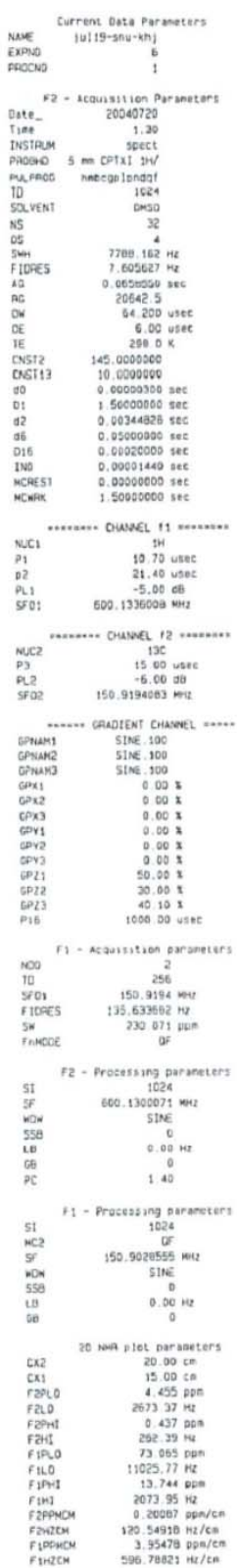


S27. Expanded HMBC (600 MHz) spectrum of halicylindramide F (1) in DMSO-d6
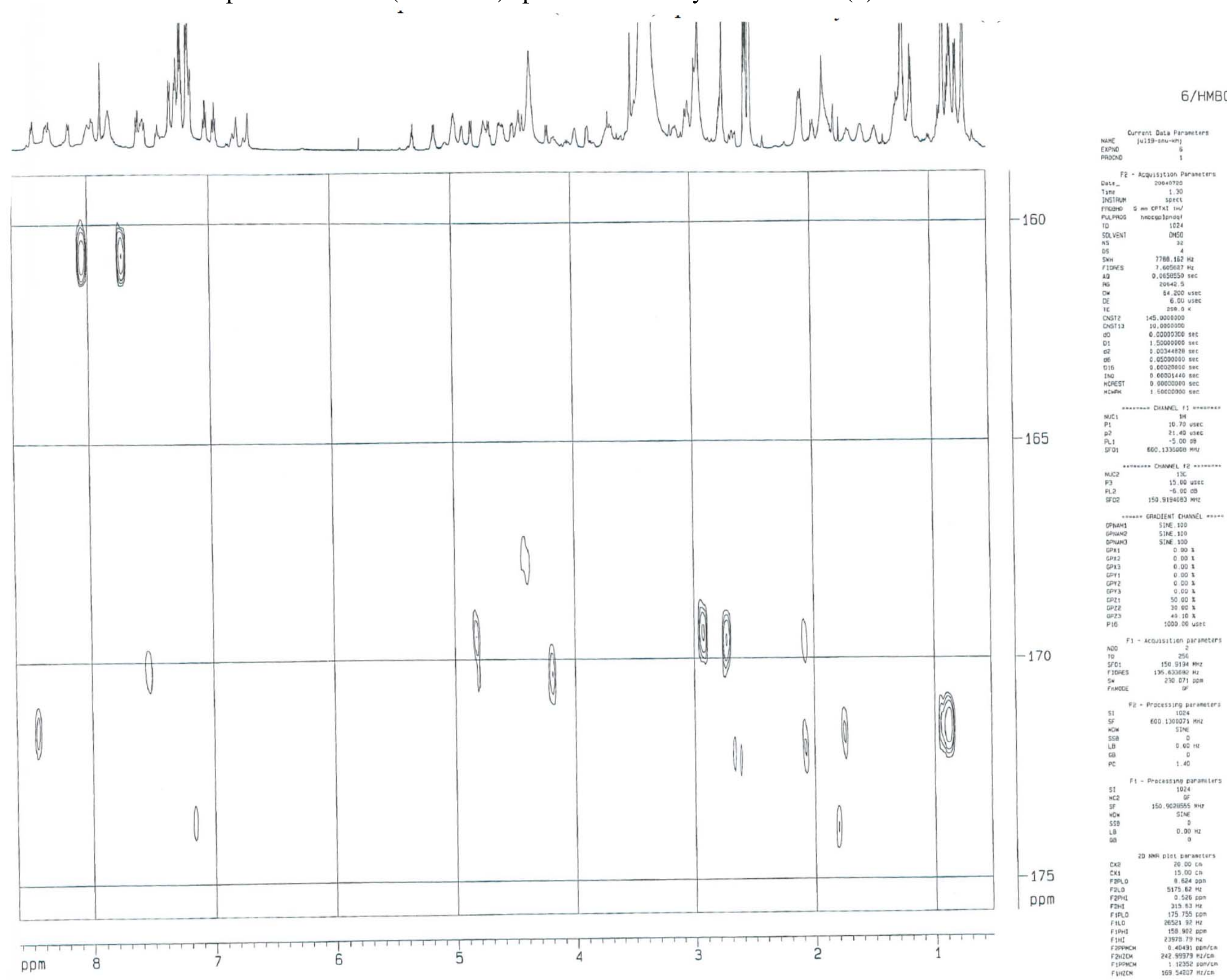
S28. HR-ESI-Tof-MS spectrum of halicylindramide G (2)
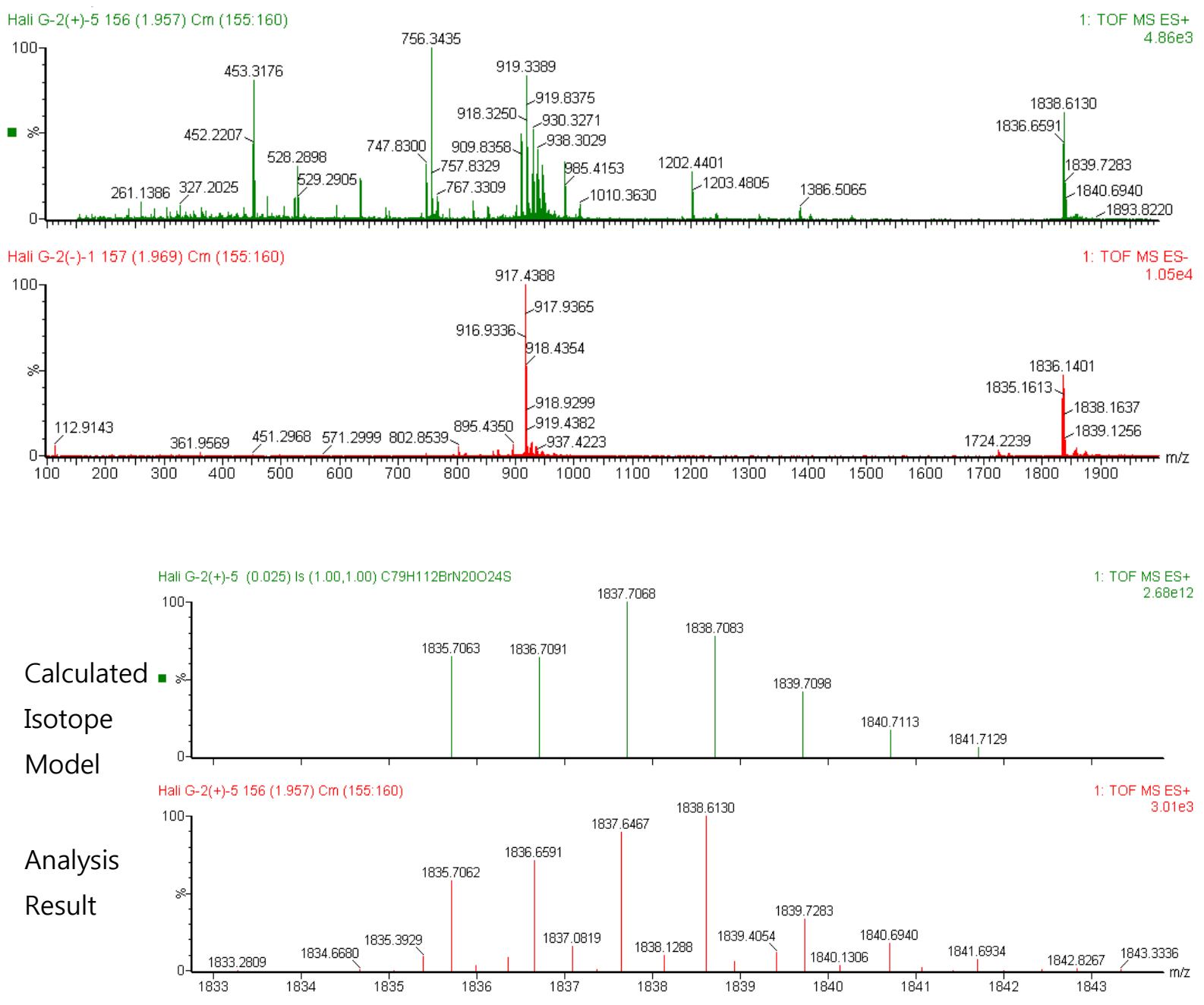

Single Mass Analysis
Tolerance $=5.0$ PPM $/$ DBE: $\min =-1.5, \max =50.0$
Selected filters: None

Even Electron lons

19 formula[e] evaluated with 2 results within limits [up to 50 best isotopic matches for each mass]

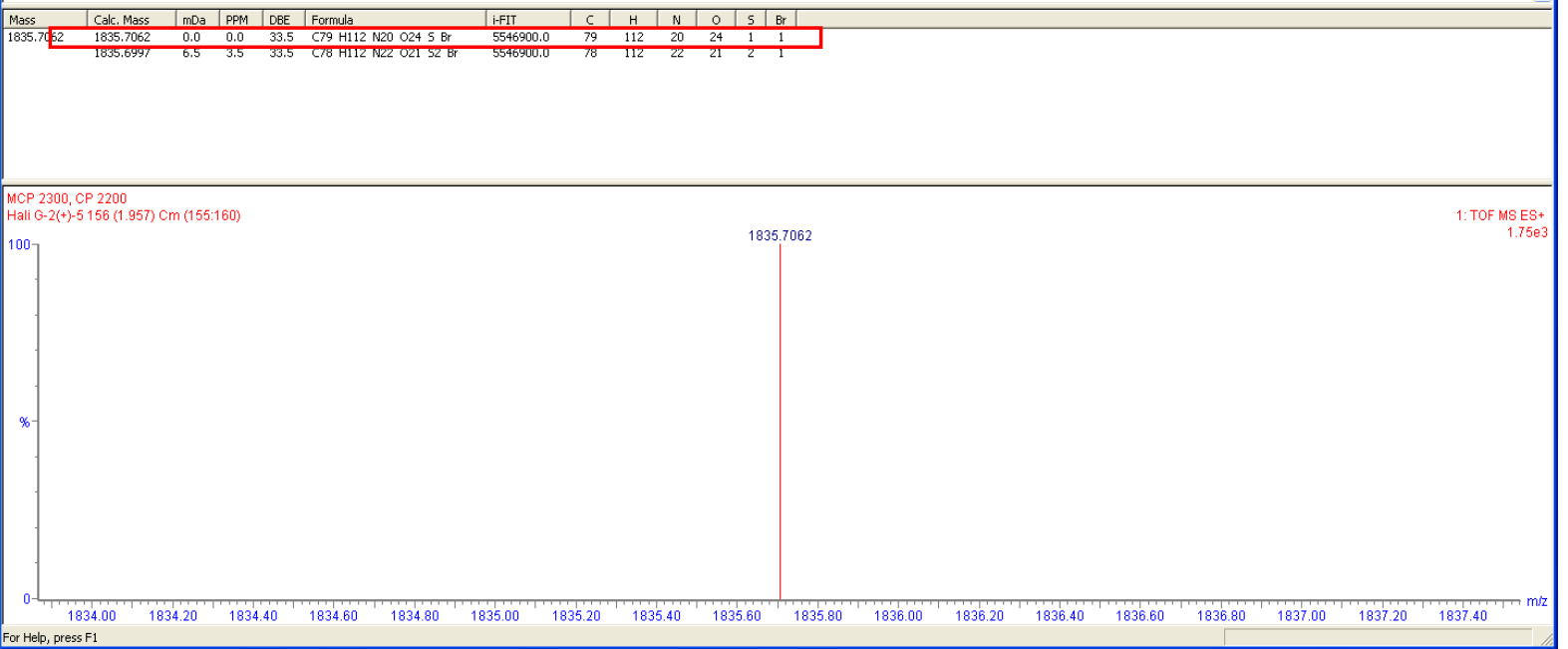


S29. 1H NMR (600 MHz) spectrum of halicylindramide G (2) in DMSO-d6

$5 / 1 \mathrm{H}$

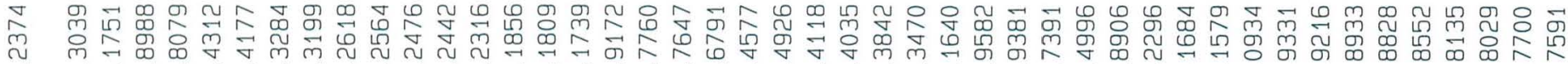
隹
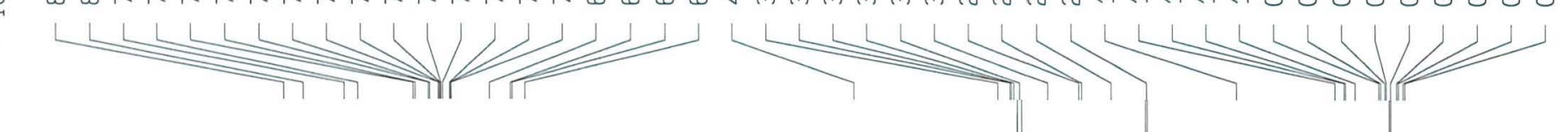

Current Data Parameters

NAME

EXPNO

PROCNO

F2 - Acquisition Parameters

Date_ 20050704

Time $\quad 16.06$

spect
INSTRUM
PROBHD $5 \mathrm{~mm} \mathrm{CPTXI} 1 \mathrm{H} /$

TD
PULPROG

SOLVENT

DS

SWH $\quad 8741.259 \mathrm{~Hz}$

FIDRES $\quad 0.133381 \mathrm{~Hz}$

AQ $\quad 3.7487664 \mathrm{sec}$

$\begin{array}{ll}\text { RG } & 20.2 \\ \text { DW } & 57.200\end{array}$

DW $\quad 57.200$ use

TE $\quad 6.00$ US

D1 100000000 sec

MCREST $\quad 1.0000000 \mathrm{sec}$

$\begin{array}{ll}\text { MCREST } & 0.00000000 \mathrm{sec} \\ \text { MCWRK } & 0.01500000 \mathrm{sec}\end{array}$

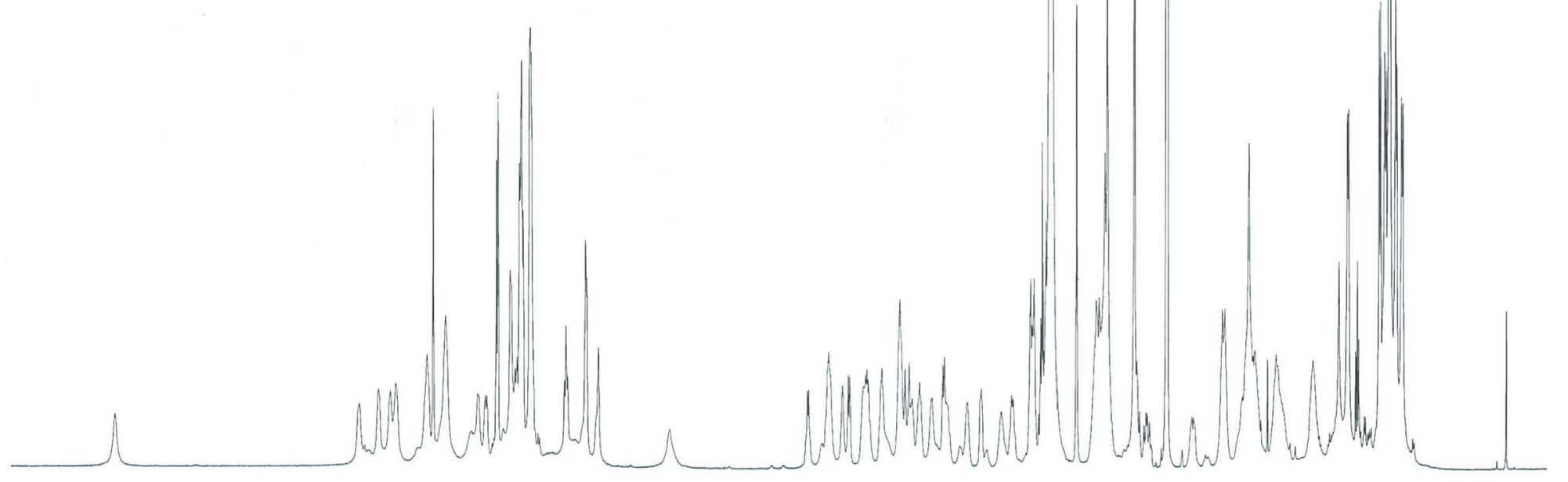

$==x==x=$ CHANNEL $f 1==z====$

$\begin{array}{lr}\text { NUC1 } & 1 \mathrm{H} \\ \text { P1 } & 10.30 \text { usec }\end{array}$

PL1 $\quad-5.00 \mathrm{~dB}$

SF01 $\quad 600.1336696 \mathrm{MHz}$

2 - Processing parameters

SI 32768

SF $\quad 600.1300074 \mathrm{MHz}$

WDW

LB $\quad 0.30 \mathrm{~Hz}$

PC

10 NMR plot parameters

CX $\quad 20.00 \mathrm{~cm}$

CY $\quad 160.00 \mathrm{~cm}$

F1P $\quad 11.000 \mathrm{ppm}$

F1 $\quad 6601.43 \mathrm{~Hz}$

$\begin{array}{ll}F 2 P & -0.300 \mathrm{ppm}\end{array}$

PPMCM $\quad 0.56500 \mathrm{ppm} / \mathrm{c}$

$\mathrm{HZCM} \quad 339.07346 \mathrm{~Hz} / \mathrm{cm}$ 


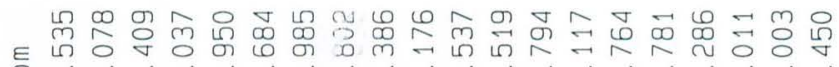

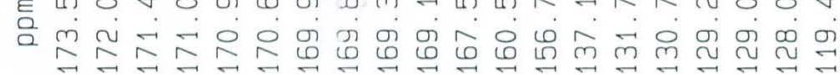

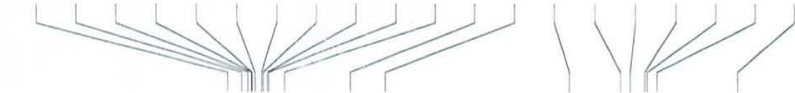

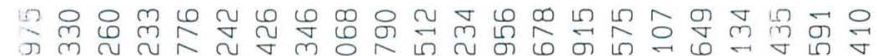

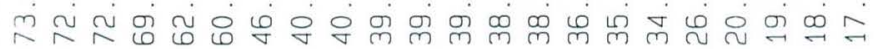
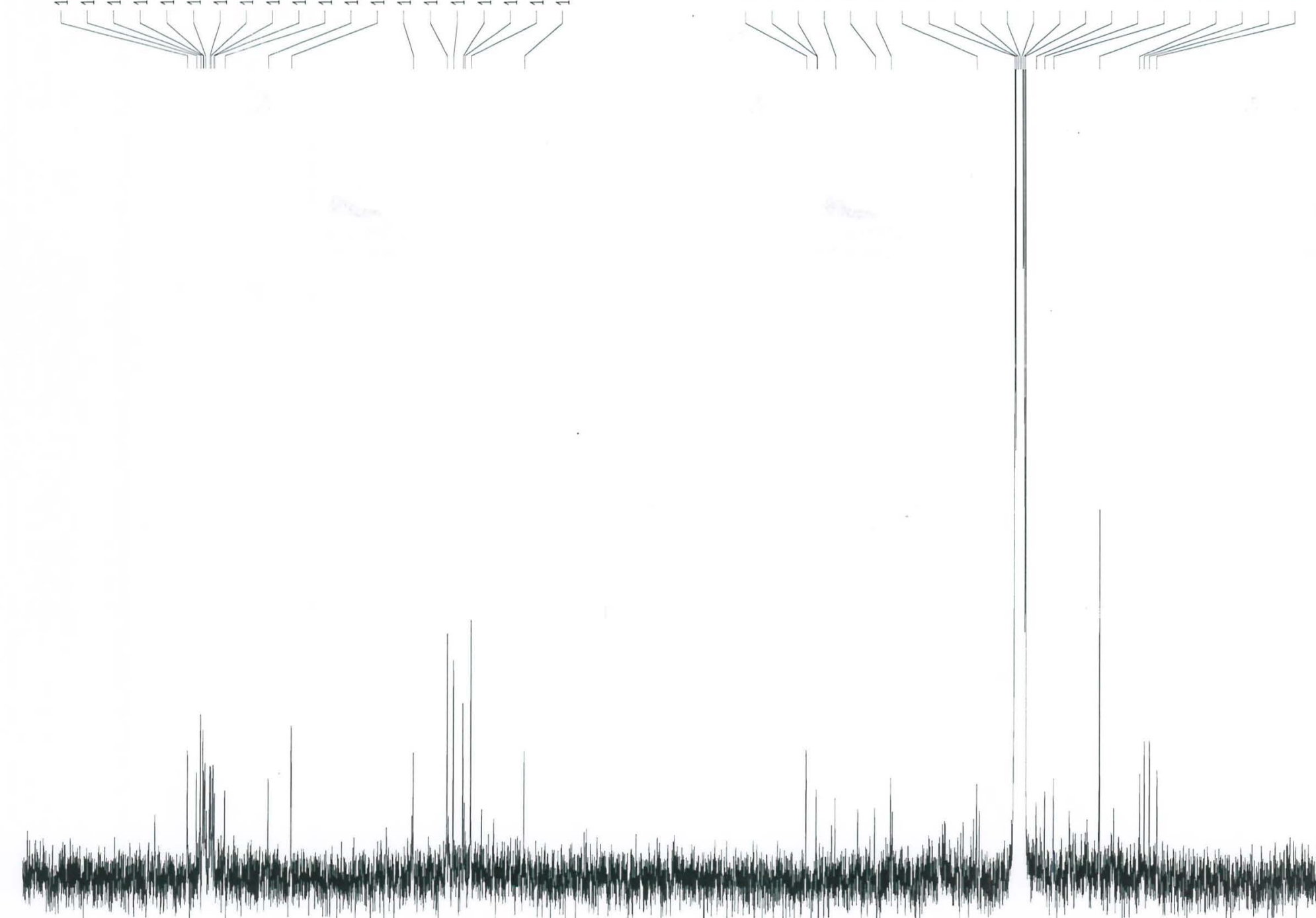

ppm

175

150

125

100
Current Data Parameters

NAME

PROCNO

=2- Acquisition Parameters

Date _ 20050706

Time

PRIRHD
INSTRM

PULPROG

TD

NS

NS

$\begin{array}{lr}\text { DS } & 0 \\ \text { SWH } & 21097.047 \mathrm{~Hz} \\ \text { FIDRES } & 0.321915 \mathrm{~Hz}\end{array}$

$\begin{array}{ll}A Q & 1.5532532 \mathrm{sec}\end{array}$

$\begin{array}{lr}\text { RG } & 8192 \\ \text { DW } & 23.700 \text { use }\end{array}$

6.00 usec $300.0 \mathrm{~K}$ $2.00000000 \mathrm{sec}$ $0.03000000 \mathrm{sec}$

D1

$\begin{array}{lr}= \pm= \pm== \pm= \pm=\text { CHANNEL } f 1 & 13 \mathrm{C} \\ \text { NUC1 } & 13 \mathrm{C} \\ \text { P1 } & 10.60 \mathrm{useC} \\ \text { PL1 } & 0.00 \mathrm{~dB} \\ \text { SF01 } & 75 . \Delta 760204 \mathrm{MHz}\end{array}$

SF01 $\quad$ 75. $4760200 \mathrm{~dB}$

$=2= \pm=m= \pm= \pm$ CHANNEL f2

CPOPAGr waltz16

$\begin{array}{ll}\text { NUCC } & 1 \mathrm{H} \\ \text { PCPD2 } & 100.00 \mathrm{usec}\end{array}$

PL2 $-5.00 \mathrm{~dB}$

$300.1312005 \mathrm{MHz}$

F2 - Processing parameter

SI 32768

SF $\quad 75.4677839 \mathrm{MHZ}$

WOW

LB $\quad 1.00 \mathrm{~Hz}$

$\begin{array}{lr}G B & 0 \\ P C & 1.40\end{array}$

10 NMR plot parameters

CX $20.00 \mathrm{~cm}$

F1P $\quad 200.000 \mathrm{ppm}$

$\begin{array}{ll}\text { F1 } & 15093.56 \mathrm{~Hz} \\ \text { F2P } & -10.000 \mathrm{pom}\end{array}$

$=2 \quad-754.68 \mathrm{~Hz}$

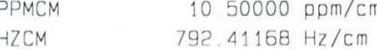


S31. HSQC (600 MHz) spectrum of halicylindramide G (2) in DMSO-d6

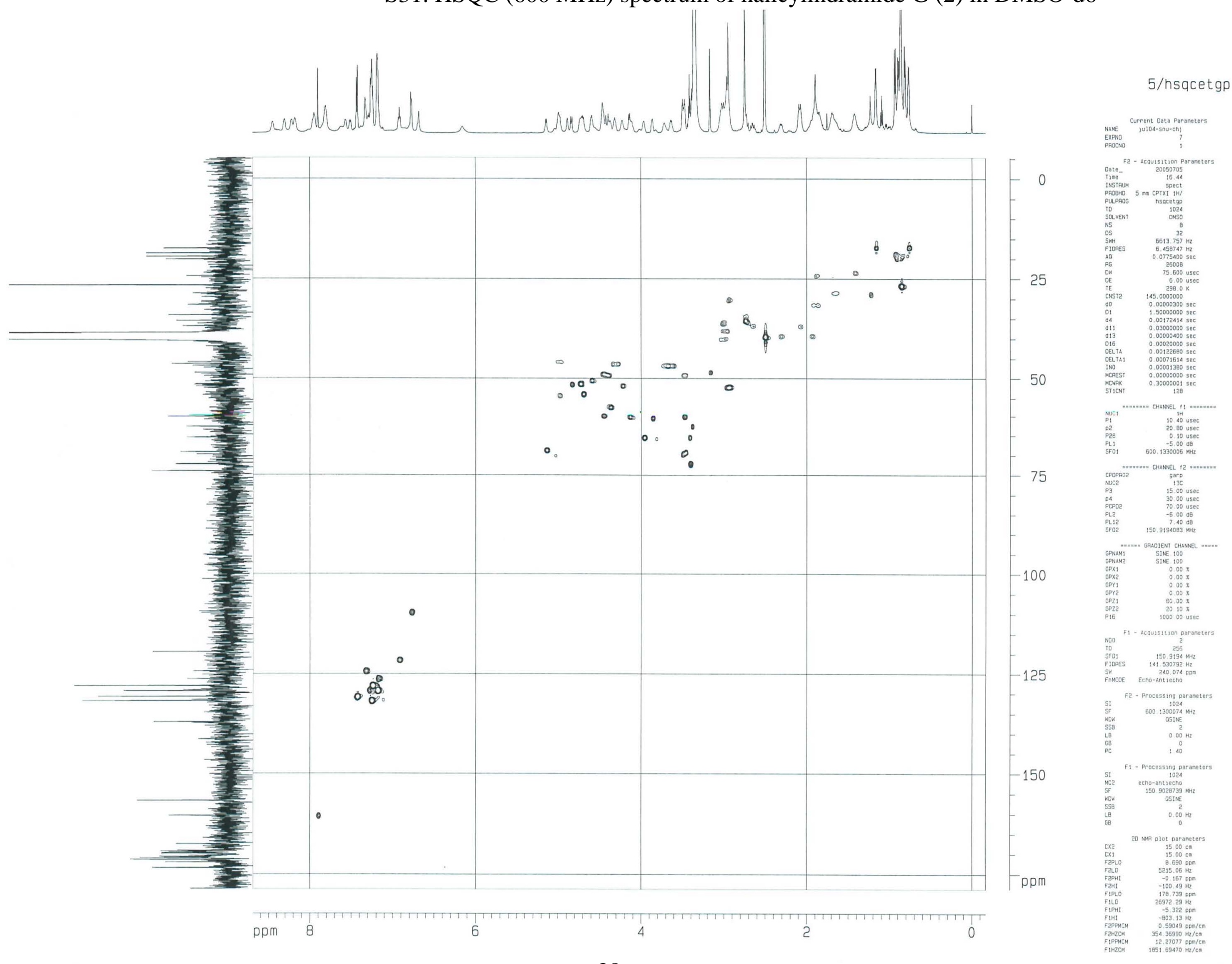


S32. COSY (600 MHz) spectrum of halicylindramide G (2) in DMSO-d6

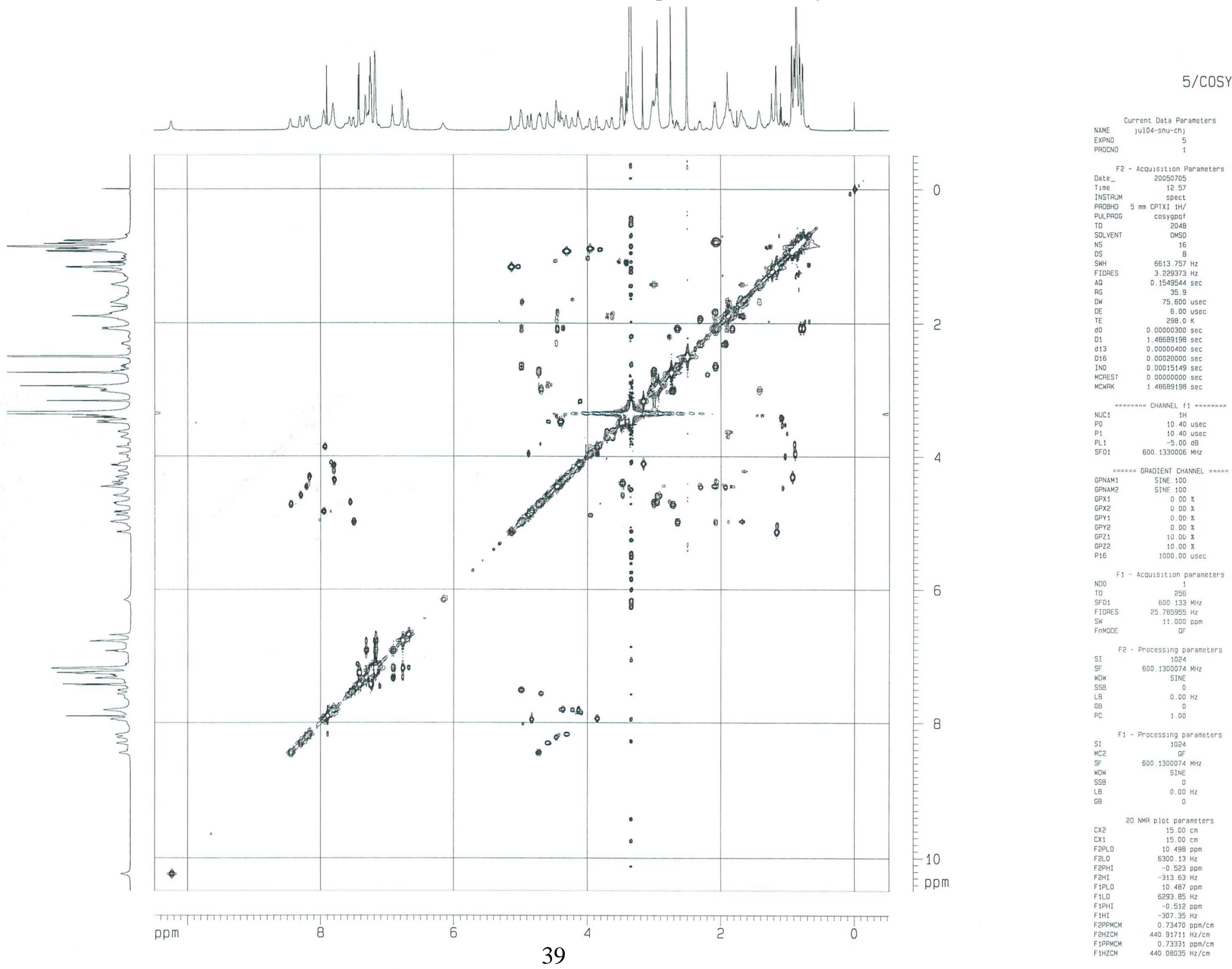


S33. Expanded COSY (600 MHz) spectrum of halicylindramide G (2) in DMSO-d6

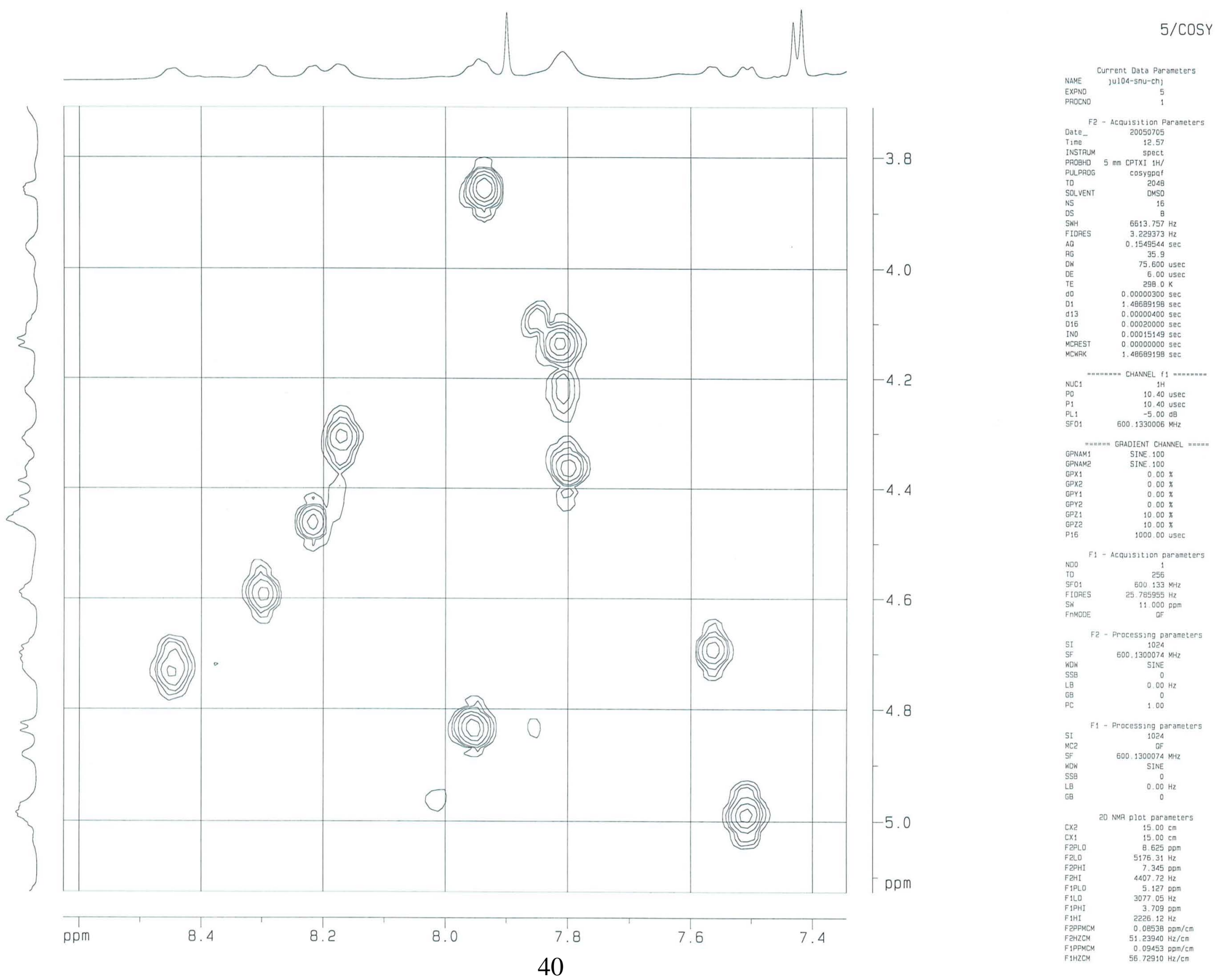


S34. Expanded COSY (600 MHz) spectrum of halicylindramide G (2) in DMSO-d6

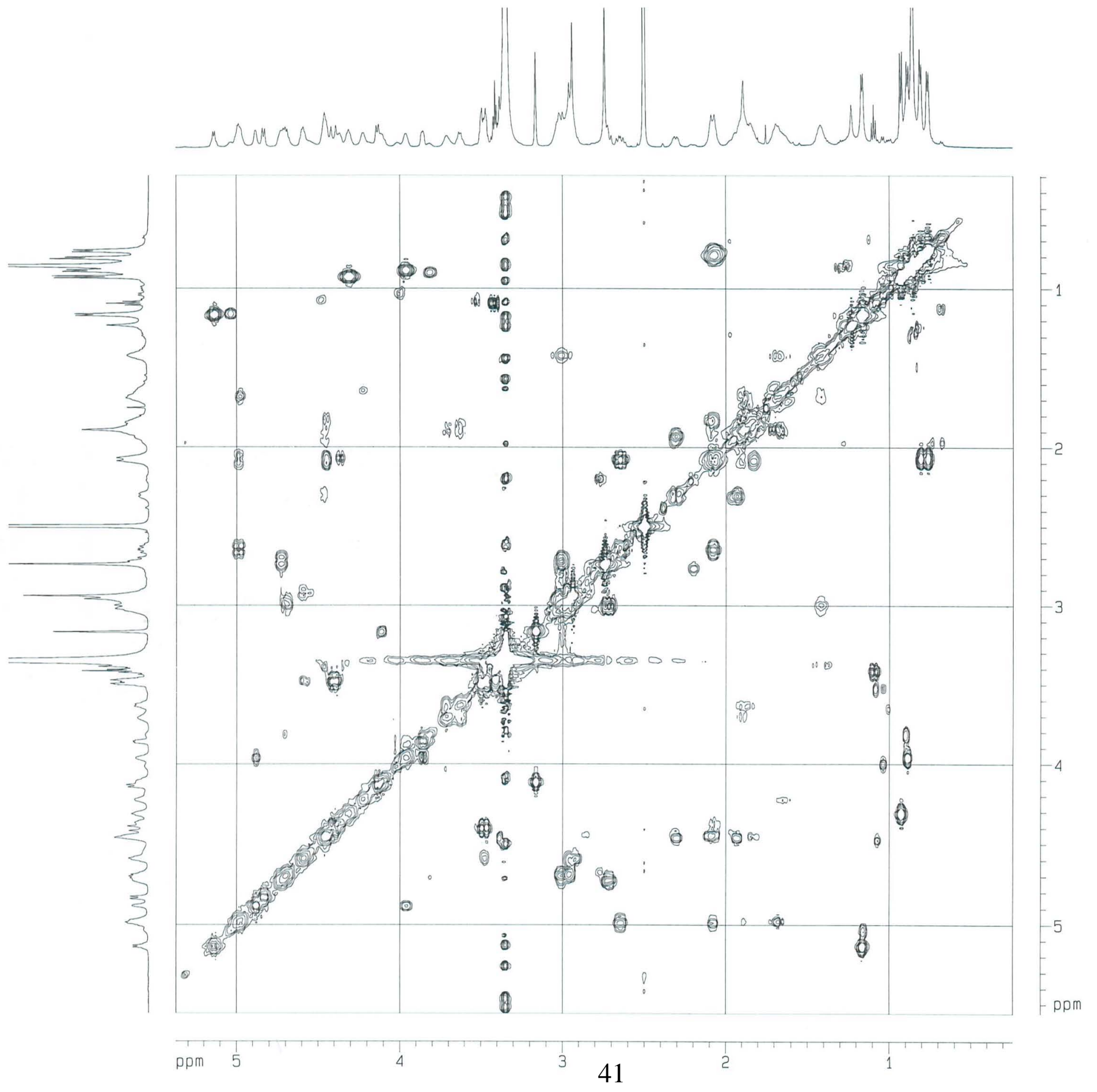

5/COSY

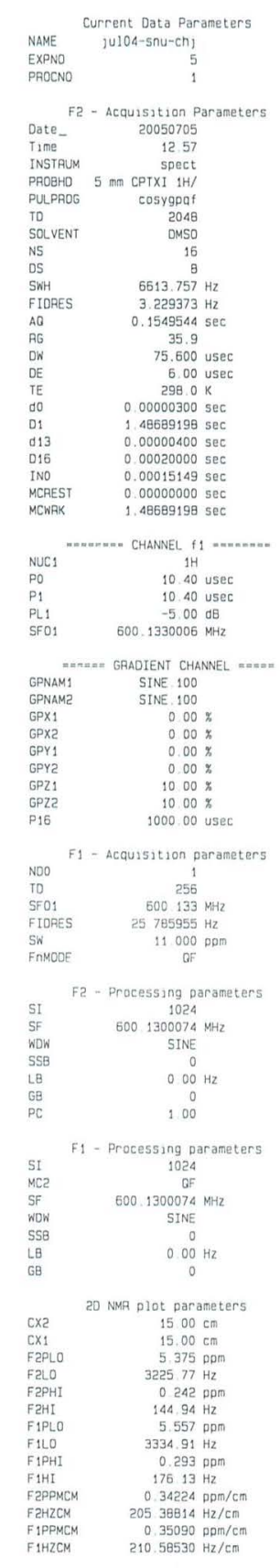


S35. TOCSY (600 MHz) spectrum of halicylindramide G (2) in DMSO-d6

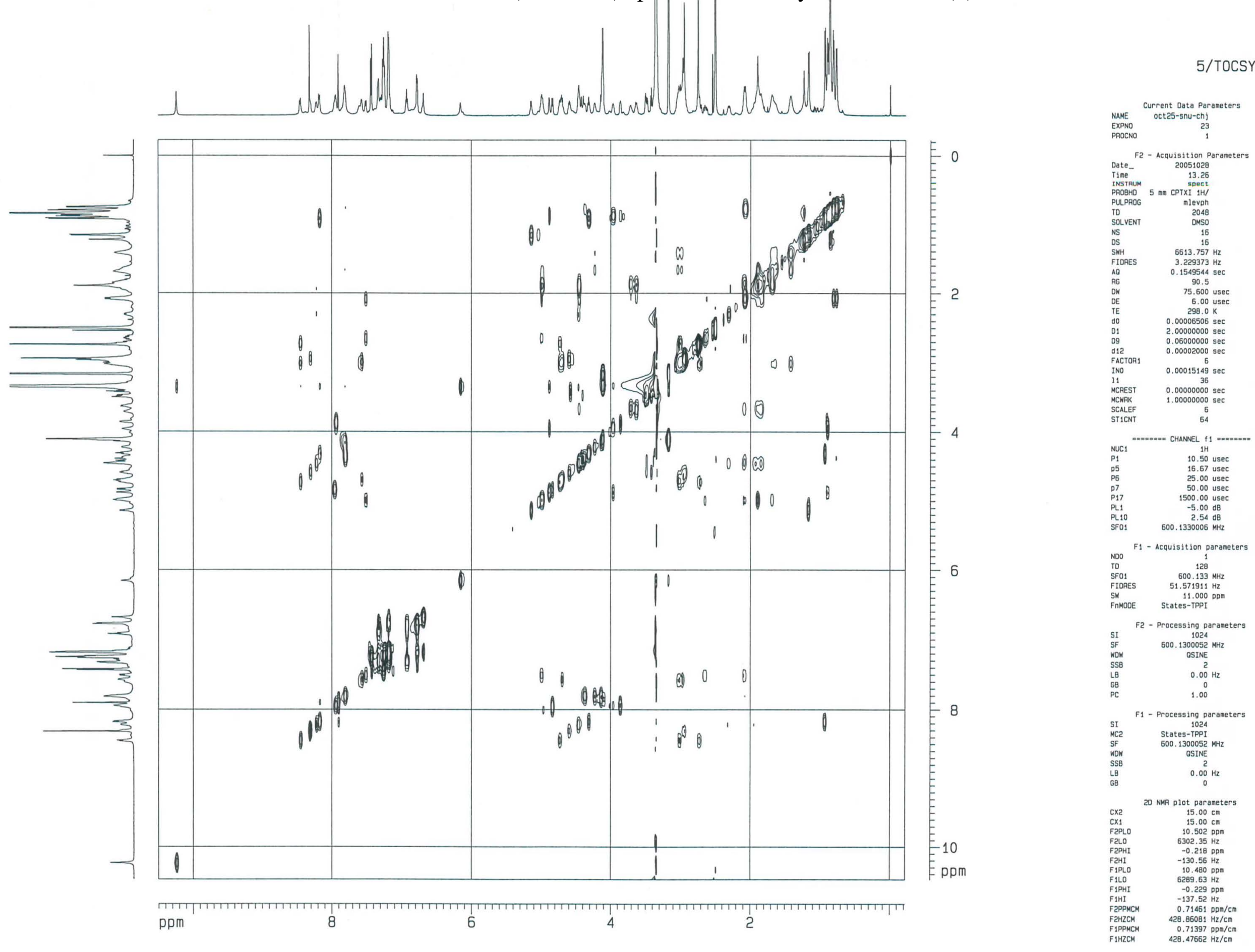


S36. Expanded TOCSY (600 MHz) spectrum of halicylindramide G (2) in DMSO-d6

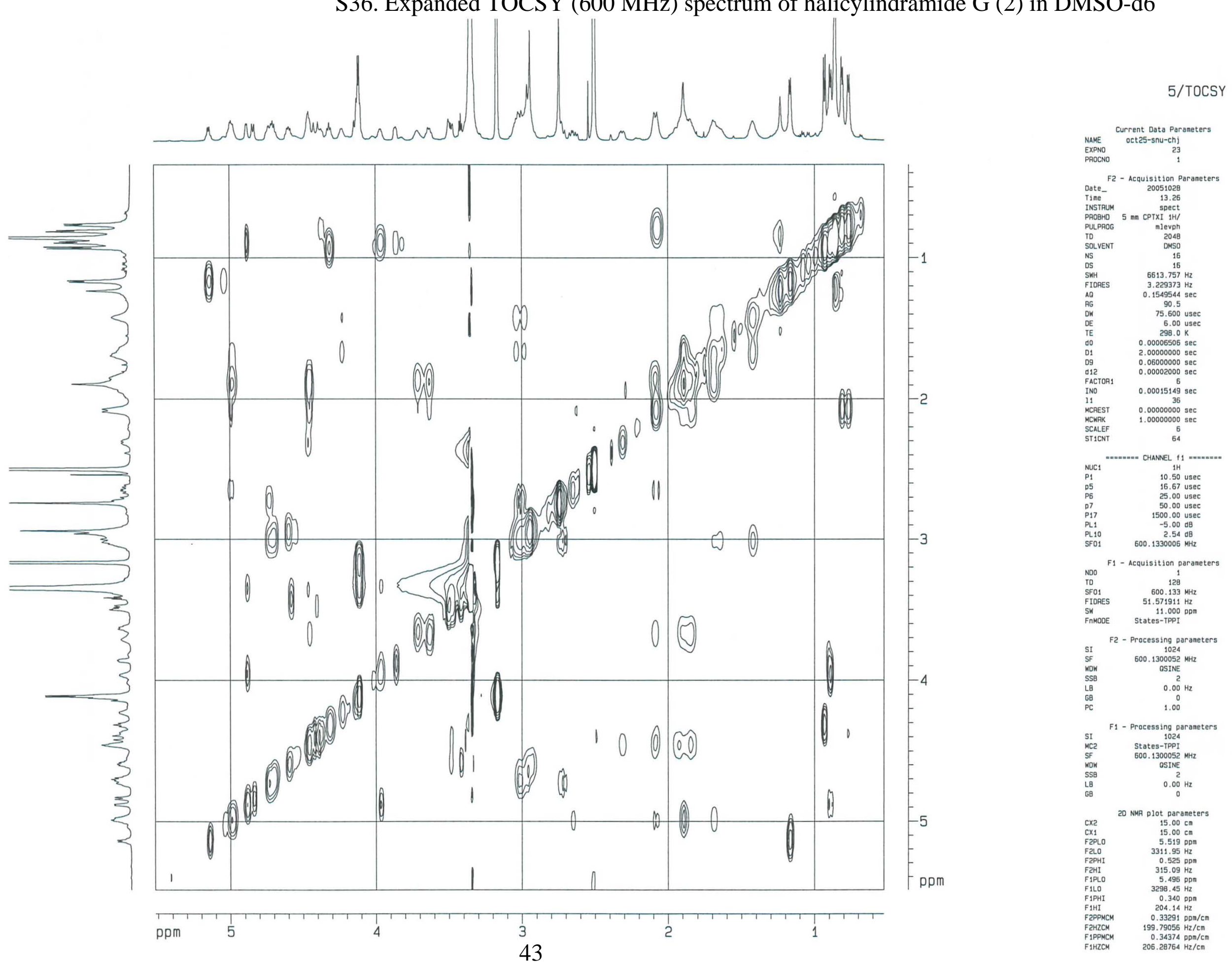


S37. Expanded TOCSY (600 MHz) spectrum of halicylindramide G (2) in DMSO-d6

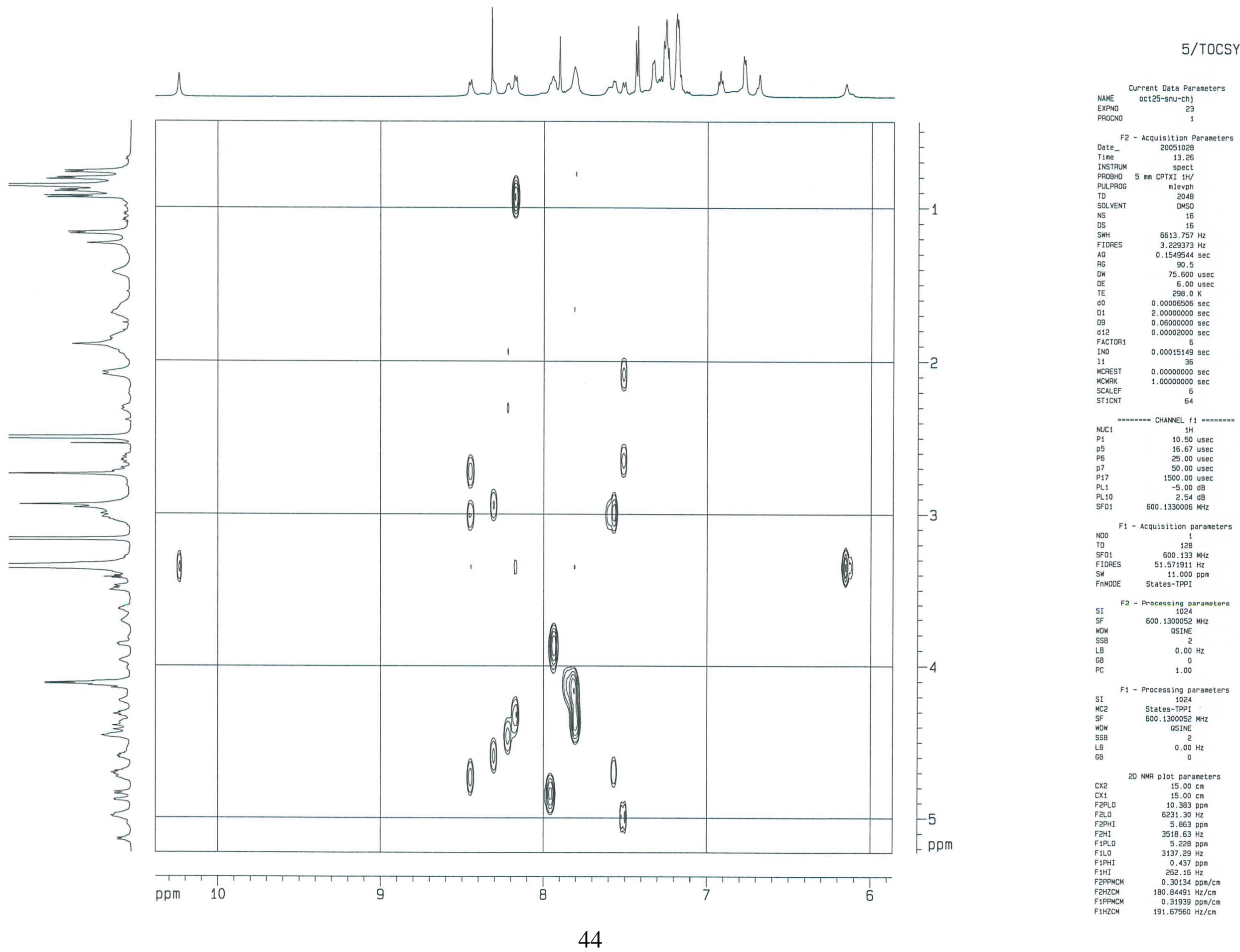


S38. Expanded TOCSY (600 MHz) spectrum of halicylindramide G (2) in DMSO-d6

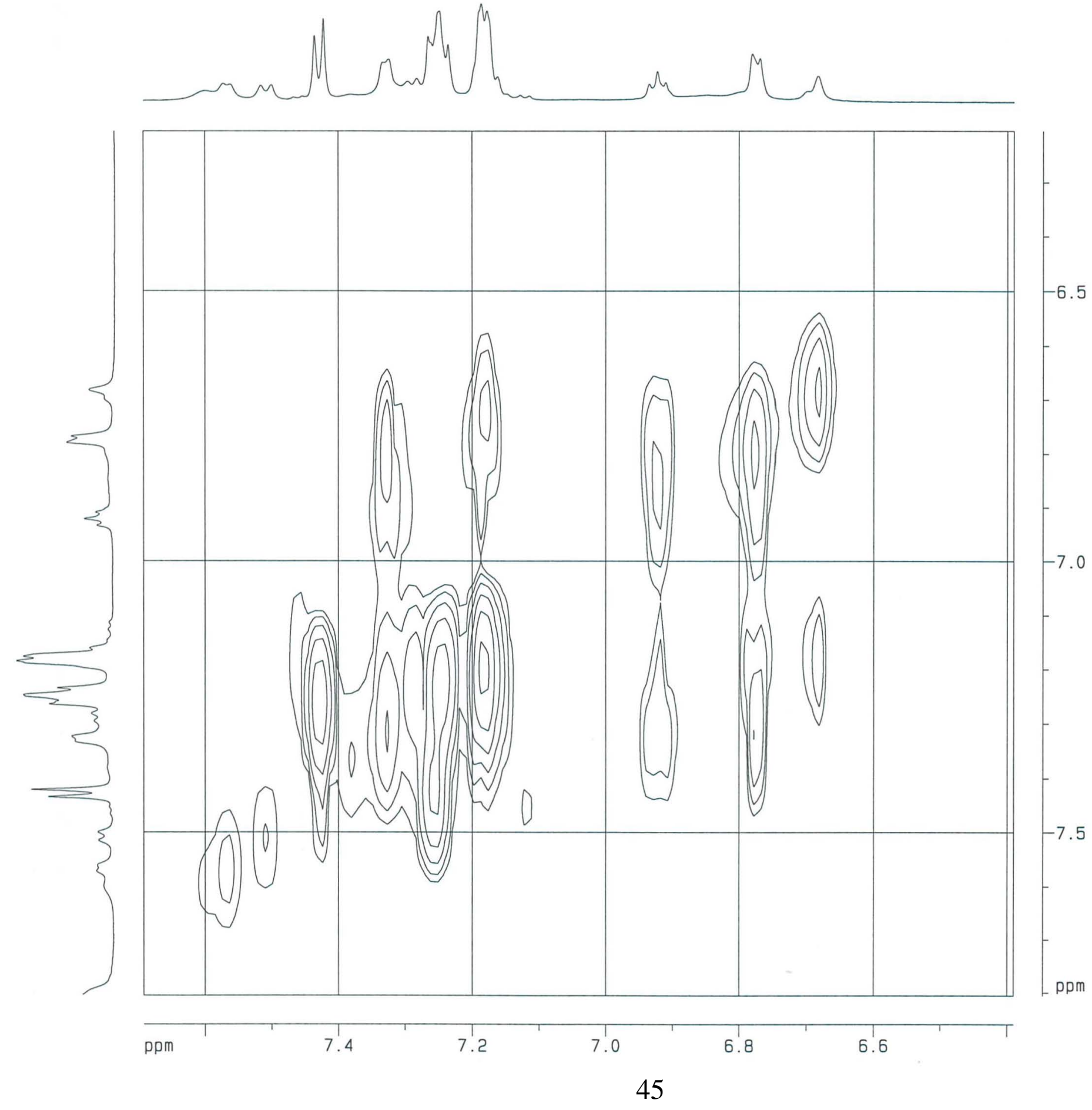

$5 /$ TOCSY

Mave Current Data Parameter

NAME
EXPNO Oct25-snu-ch

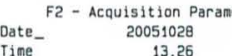

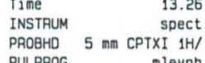

$\begin{array}{lr}\text { TD } & 2048 \\ \text { Solvent } & \text { DMSO } \\ \text { NS } & 16 \\ \text { NS } & 16\end{array}$

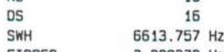

$\begin{array}{lc}\text { FIDAES } & 3.229373 \mathrm{~Hz} \\ \text { AO } & 0.1549544 \mathrm{sec} \\ \text { A6 } & 90.5\end{array}$

$\begin{array}{lrl}\text { OH } & 75.500 \mathrm{usec} \\ \text { OE } & 6.00 \mathrm{usec} \\ \text { OE } & 298.0 \mathrm{~K}\end{array}$

$\begin{array}{ll}d 0 & 0.000005506 \mathrm{sec} \\ 01 & 2.000000000 \mathrm{sec} \\ 09 & 0.05000000\end{array}$

$\begin{array}{ll}012 & 0.06000000 \mathrm{sec} \\ \text { FACTOP } & 0.00002000 \mathrm{sec}\end{array}$

${ }_{11}^{\text {INO }} \quad 0.00015149 \mathrm{sec}$

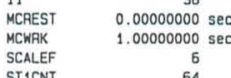

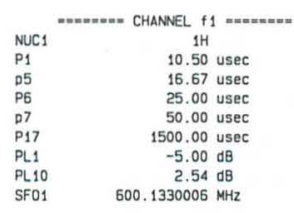

F1 - Acquisition parameters

$\begin{array}{lr}\text { TD } & 128 \\ \text { SF01 } & 600.133 \mathrm{MHz} \\ \text { FIDRES } & 51.571911 \mathrm{~Hz}\end{array}$

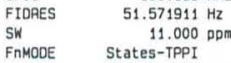

F2 - Processing parameters

$\begin{array}{lc}\text { SI } & 1024 \\ \text { SF } & 600.1300052 \text { MHZ } \\ \text { HOW } & \text { OSINE }\end{array}$

$\begin{array}{ll}\text { SSB } & 2 \\ 58 & 0.00 \mathrm{H} \\ 6 \mathrm{~B} & 0 \\ \mathrm{GC} & 1.00\end{array}$

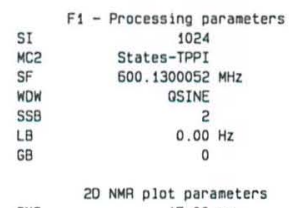

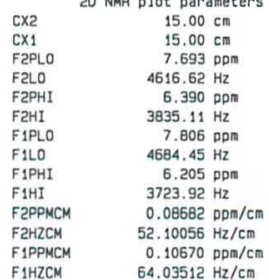


S39. NOESY (600 MHz) spectrum of halicylindramide G (2) in DMSO-d6

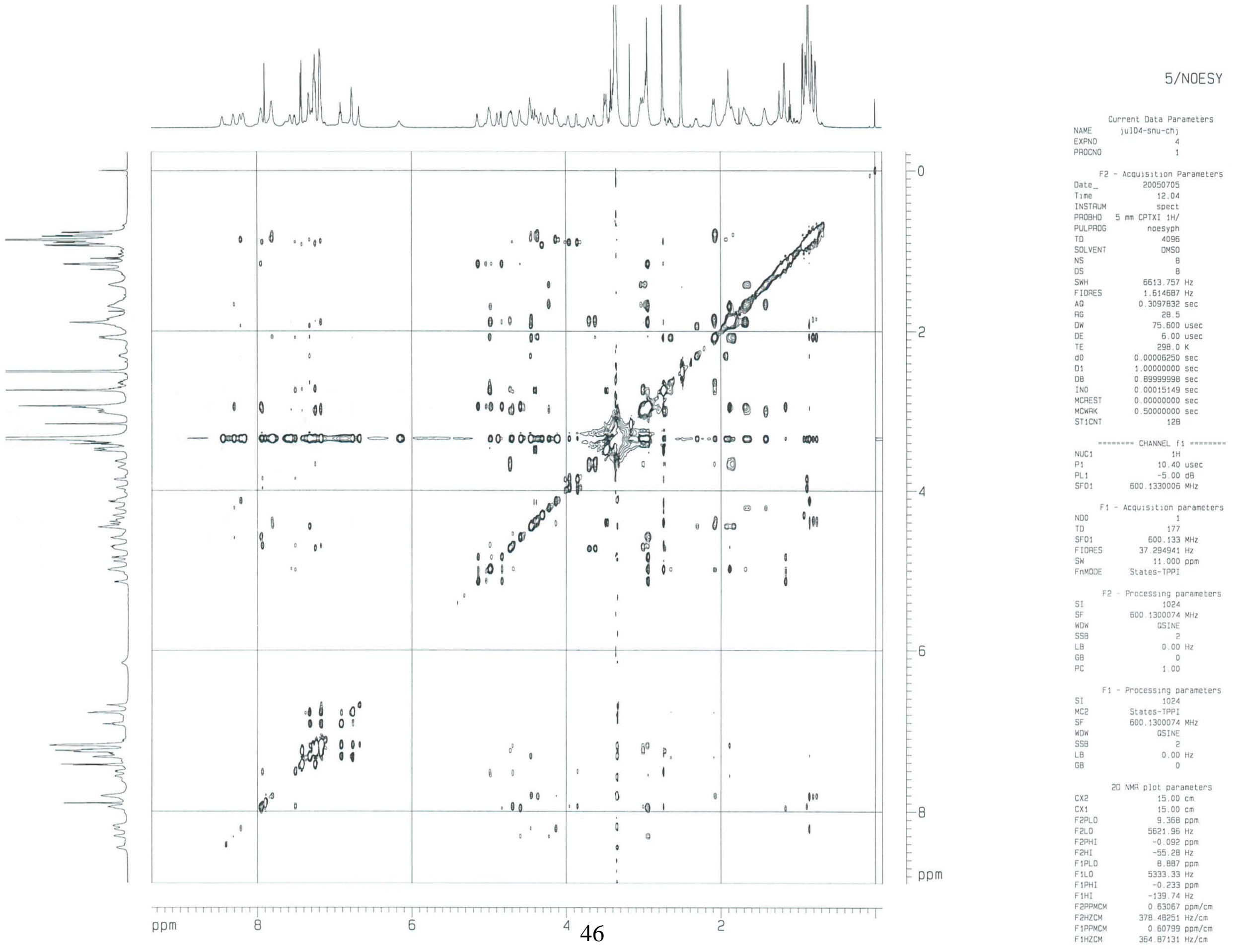


S40. Expanded NOESY (600 MHz) spectrum of halicylindramide G (2) in DMSO-d6

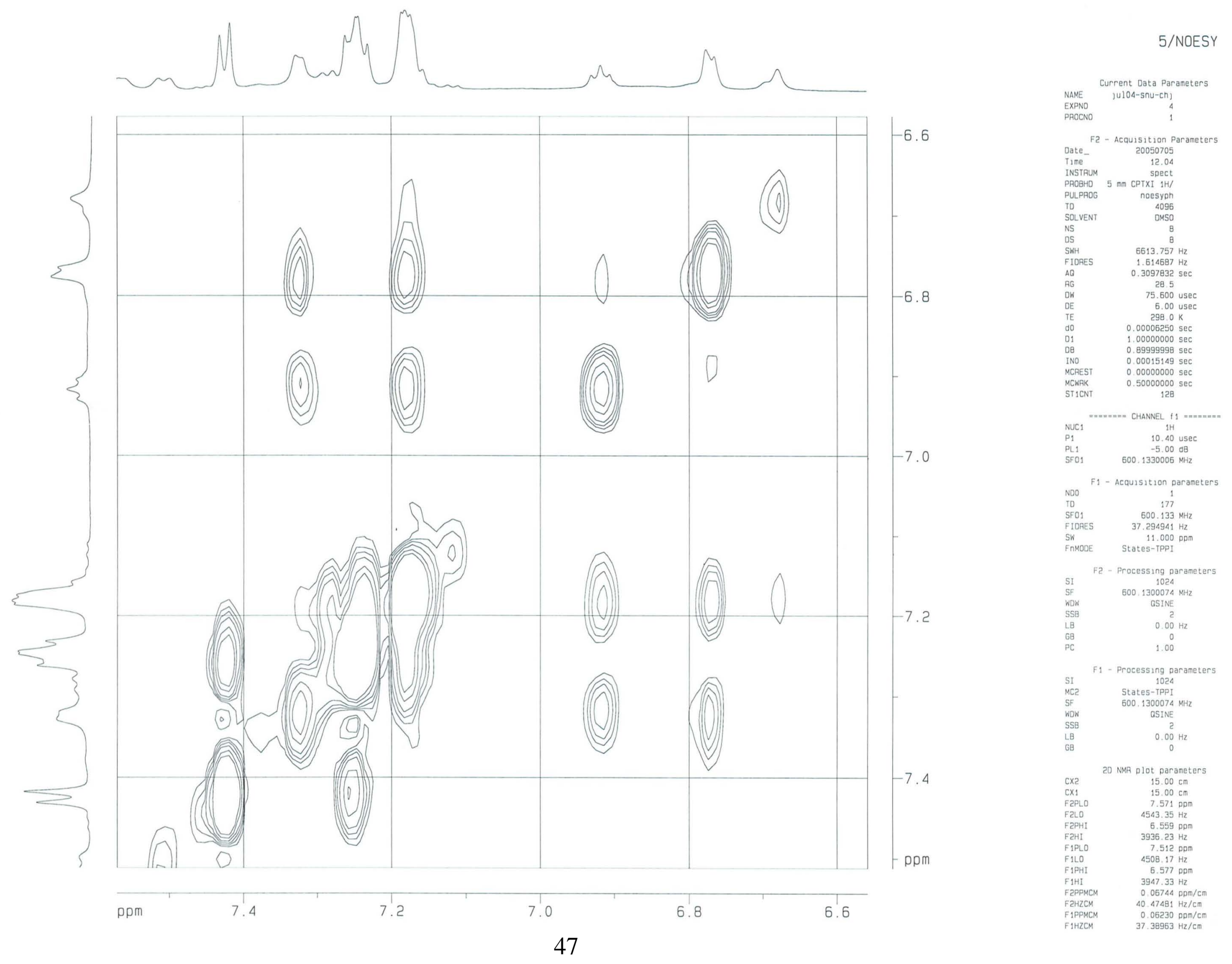


S41. Expanded NOESY (600 MHz) spectrum of halicylindramide G (2) in DMSO-d6

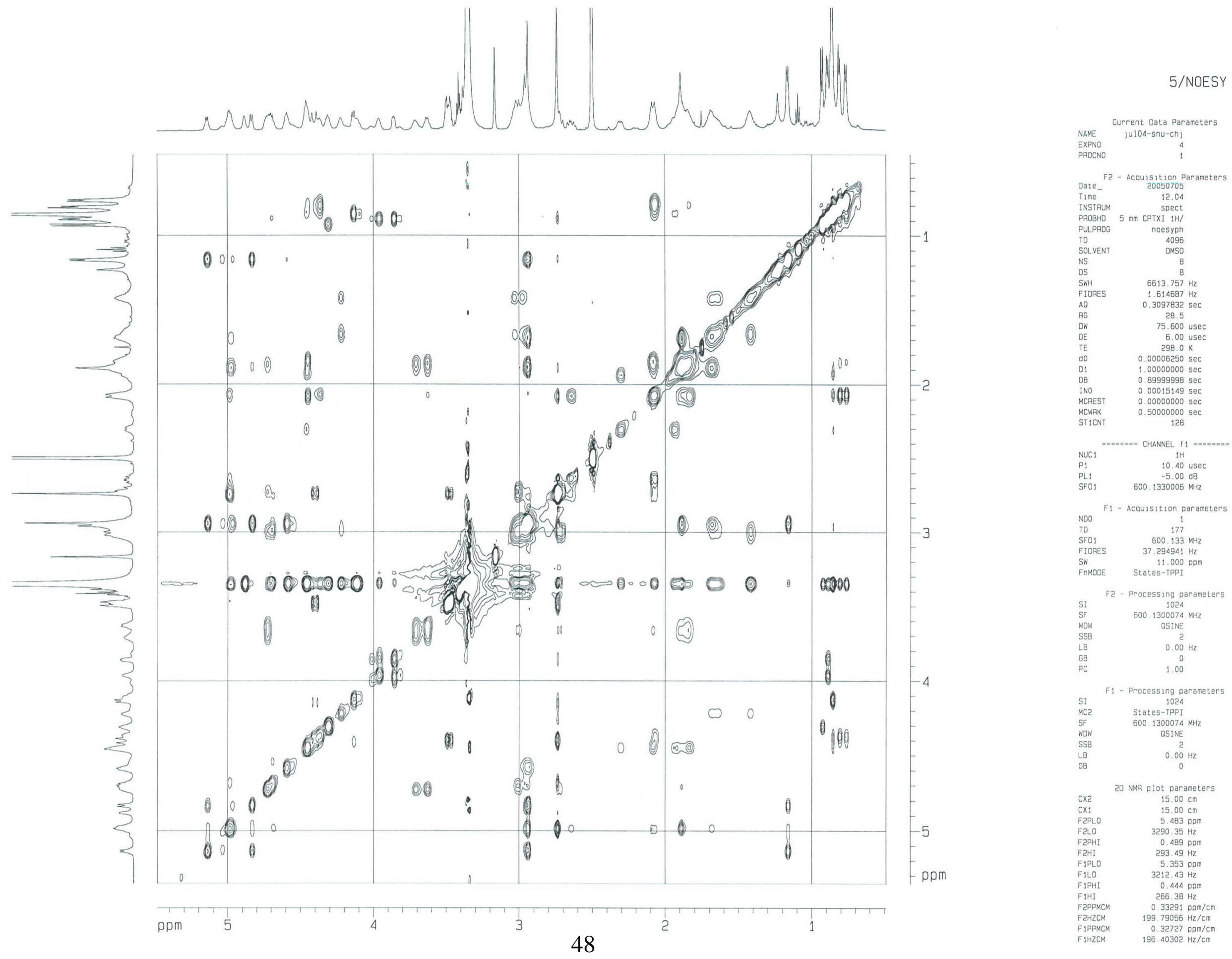


S42. Expanded NOESY (600 MHz) spectrum of halicylindramide G (2) in DMSO-d6

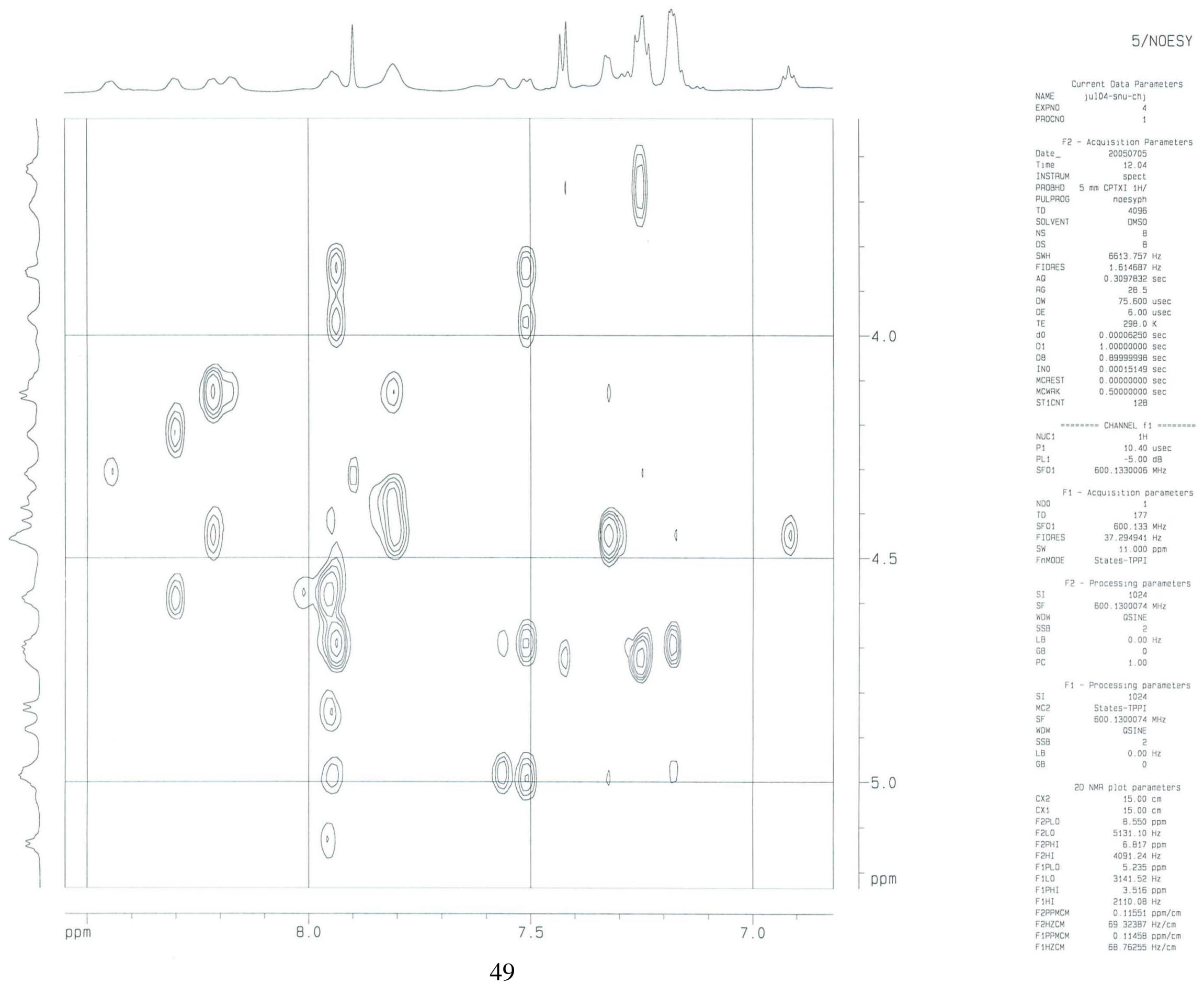


S43. Expanded NOESY (600 MHz) spectrum of halicylindramide G (2) in DMSO-d6

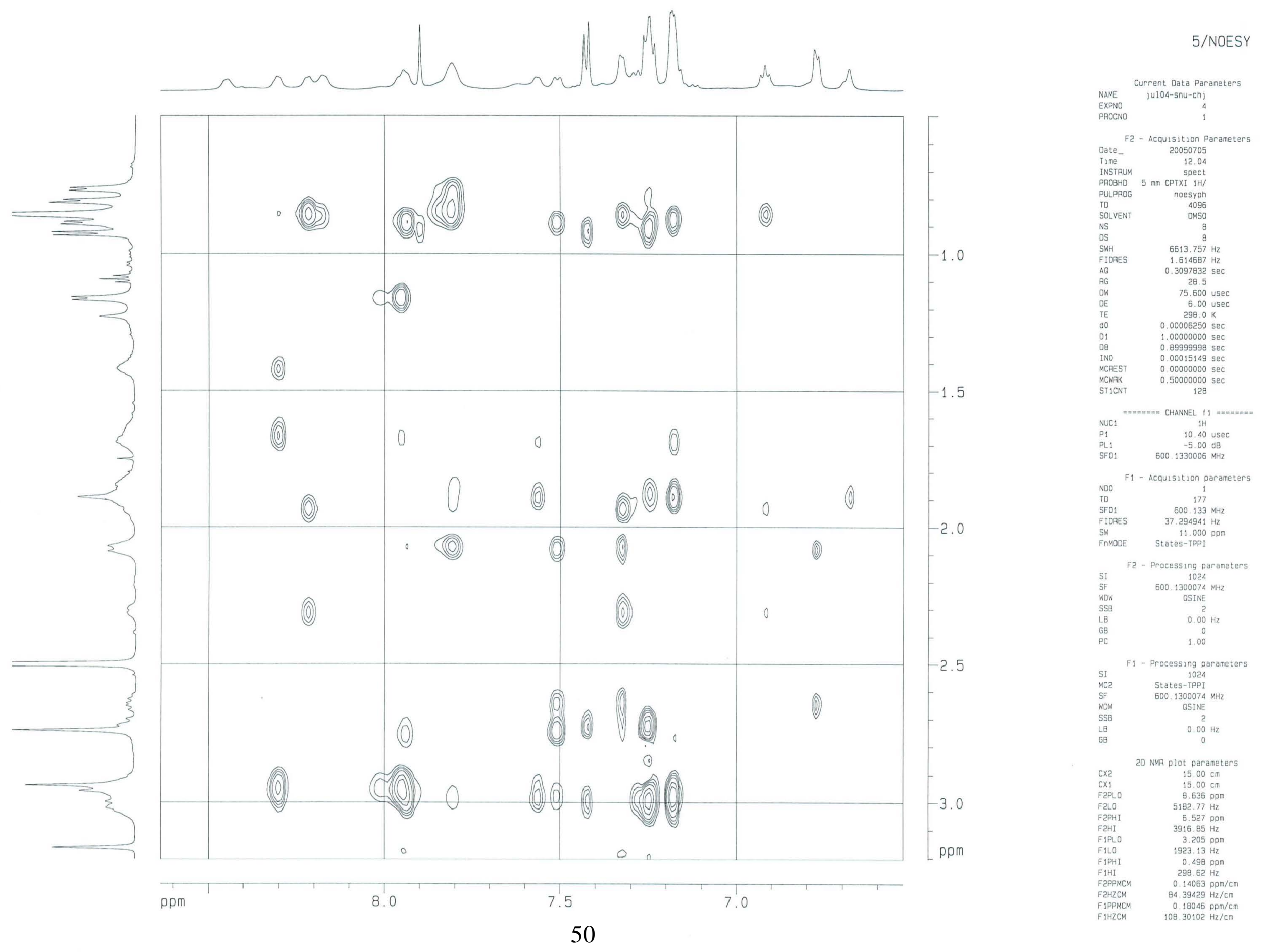


S44. HMBC (600 MHz) spectrum of halicylindramide G (2) in DMSO-d6

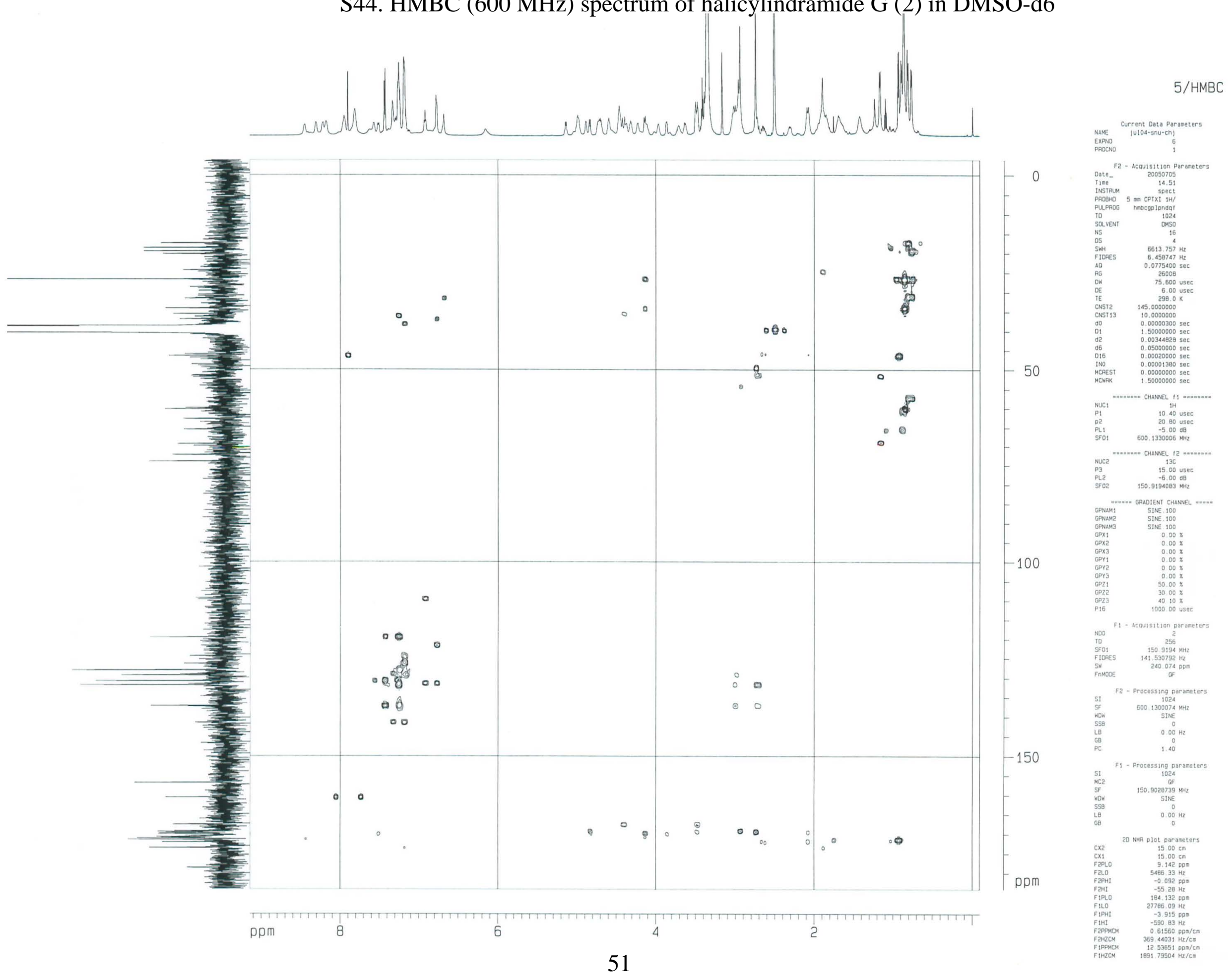


S45. Expanded HMBC (600 MHz) spectrum of halicylindramide G (2) in DMSO-d6

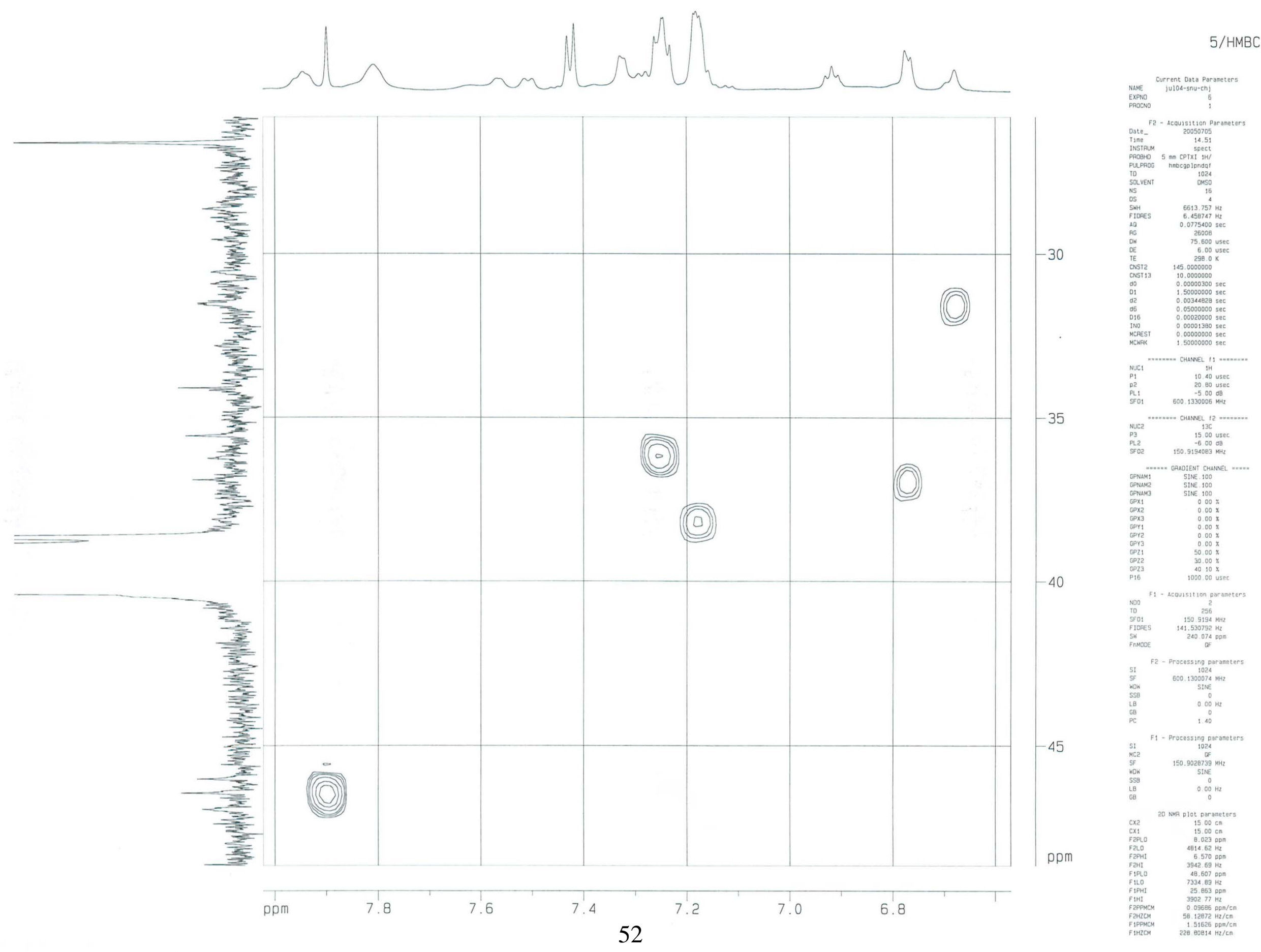


S46. Expanded HMBC (600 MHz) spectrum of halicylindramide G (2) in DMSO-d6

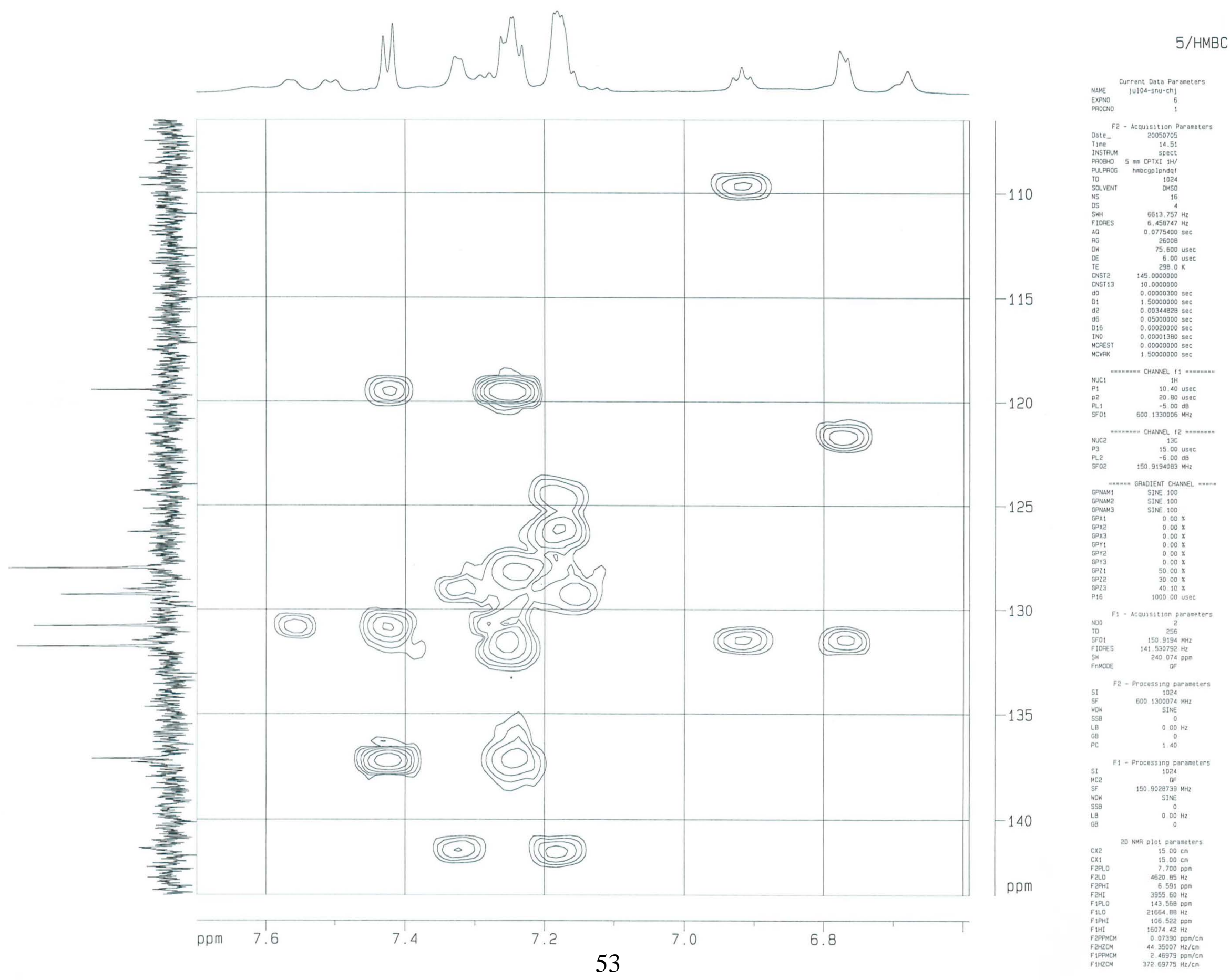


S47. Expanded HMBC (600 MHz) spectrum of halicylindramide G (2) in DMSO-d6

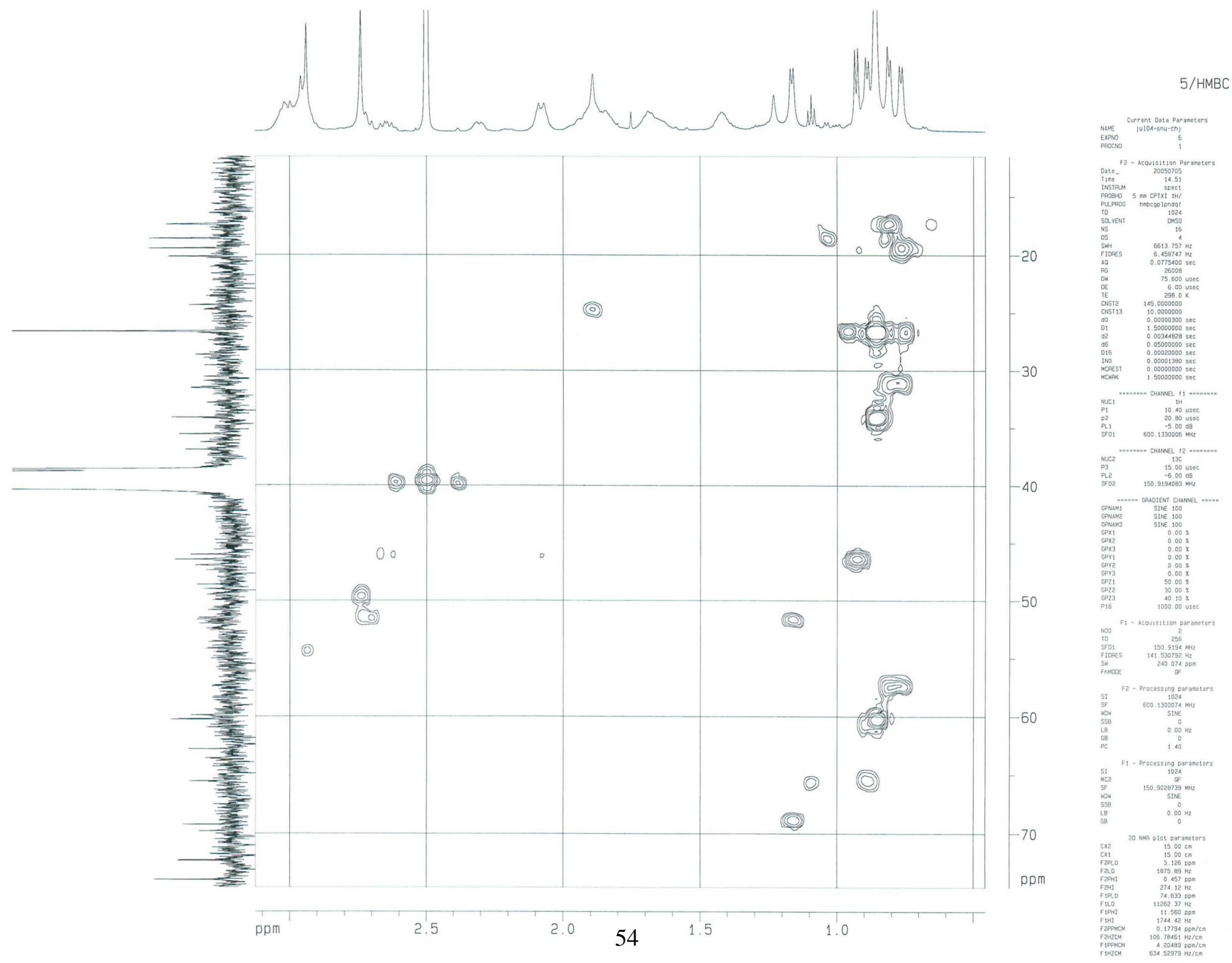


S48. Expanded HMBC (600 MHz) spectrum of halicylindramide G (2) in DMSO-d6

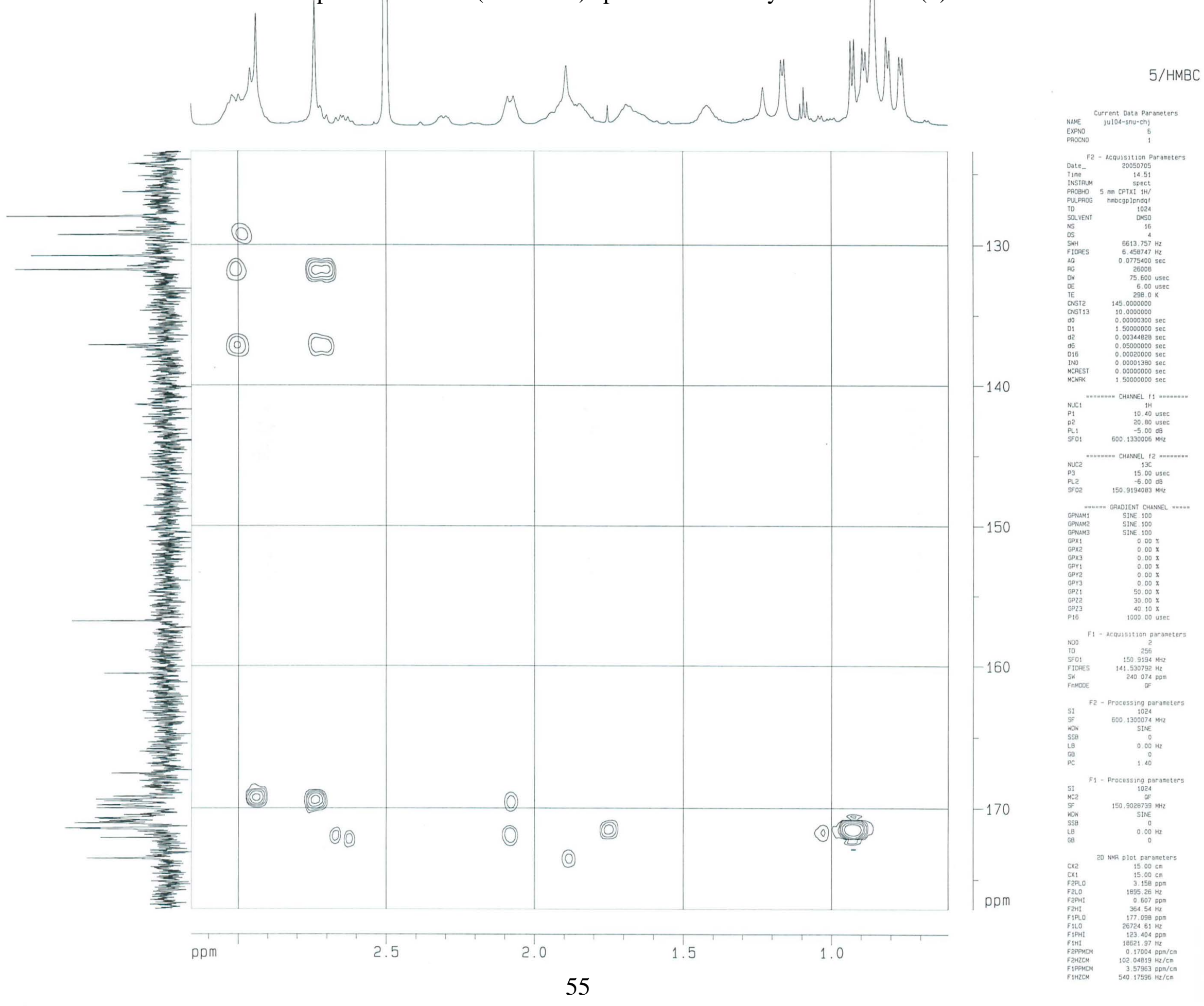


S49. Expanded HMBC (600 MHz) spectrum of halicylindramide G (2) in DMSO-d6

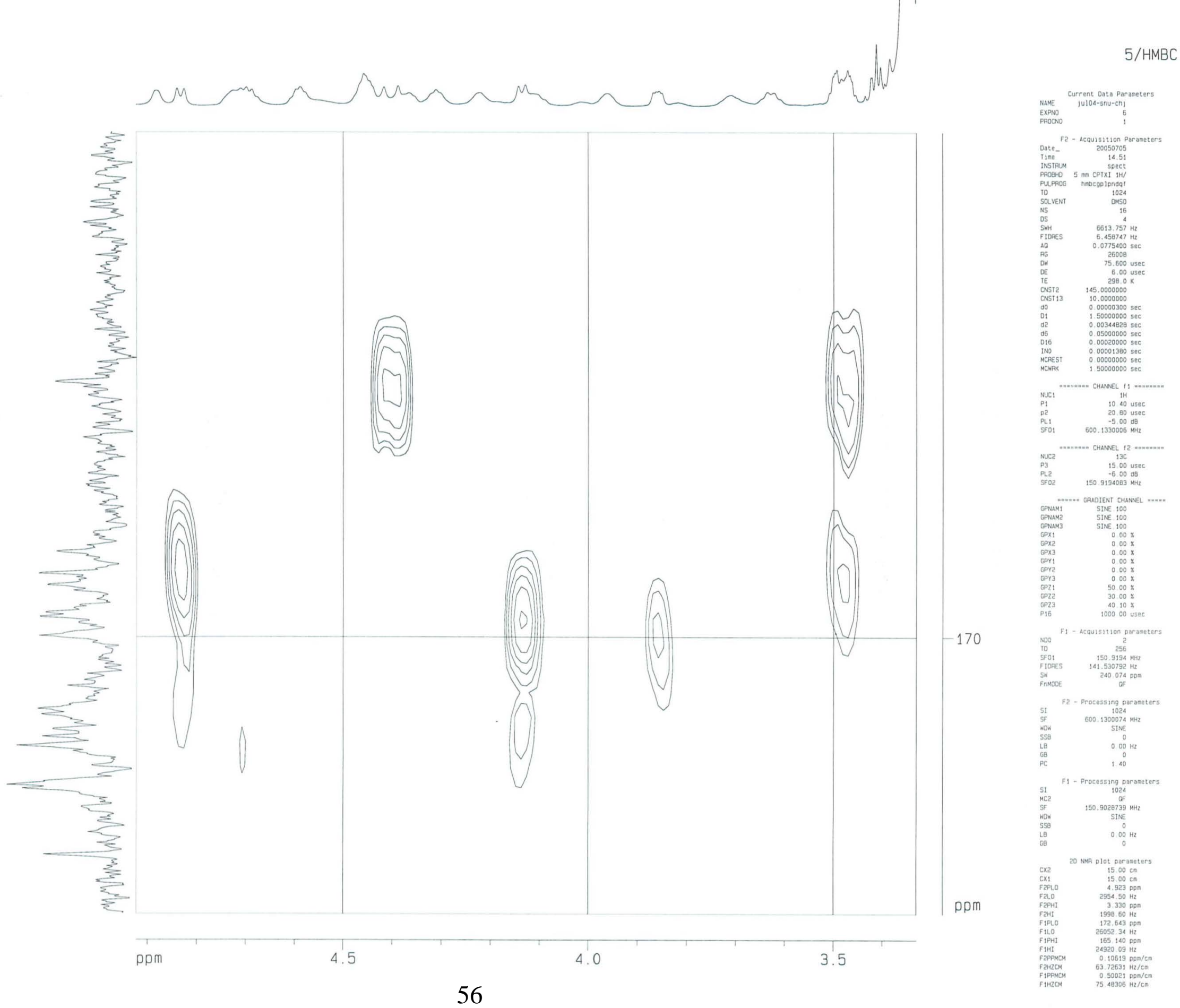


S50. Expanded HMBC (600 MHz) spectrum of halicylindramide G (2) in DMSO-d6

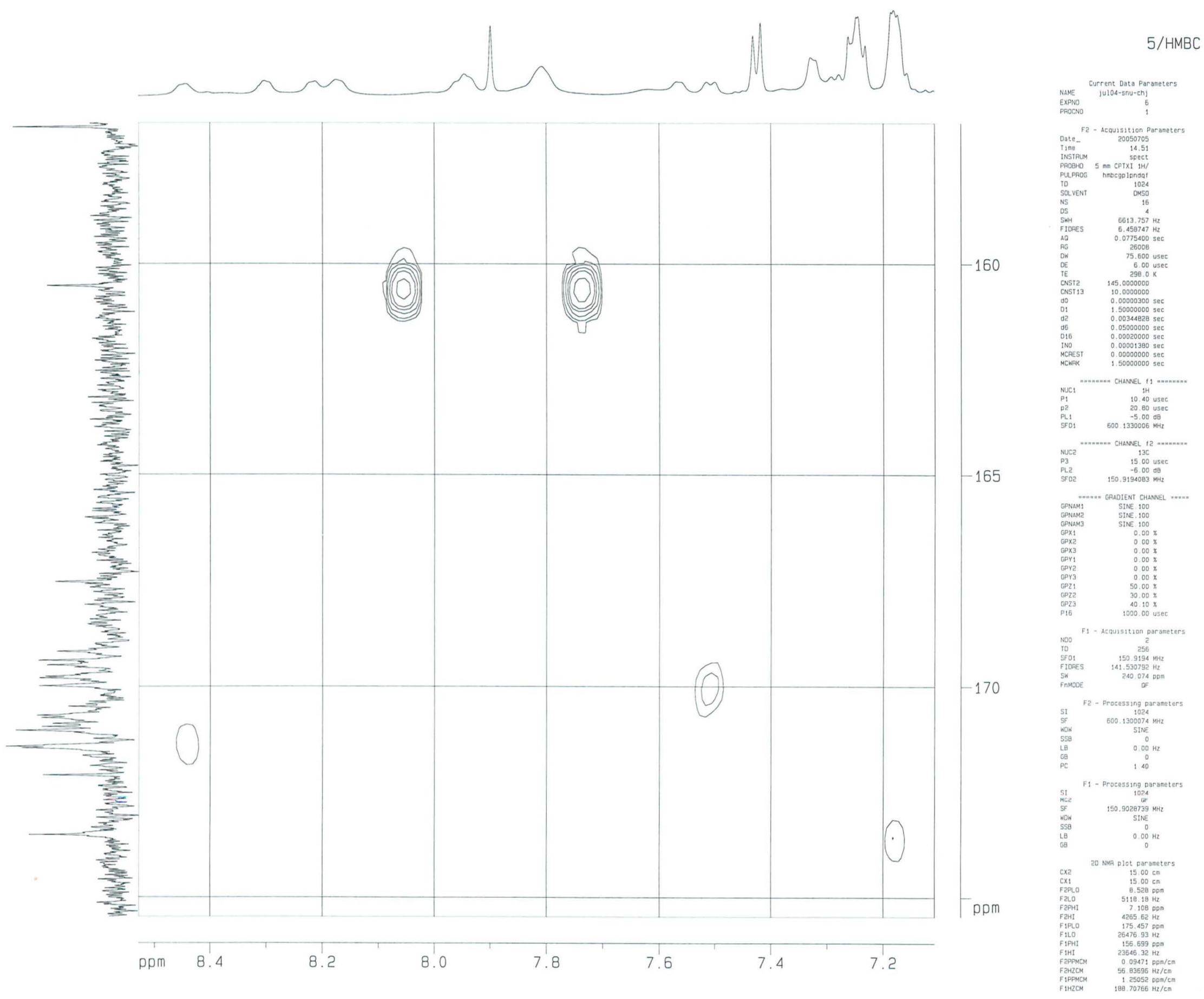




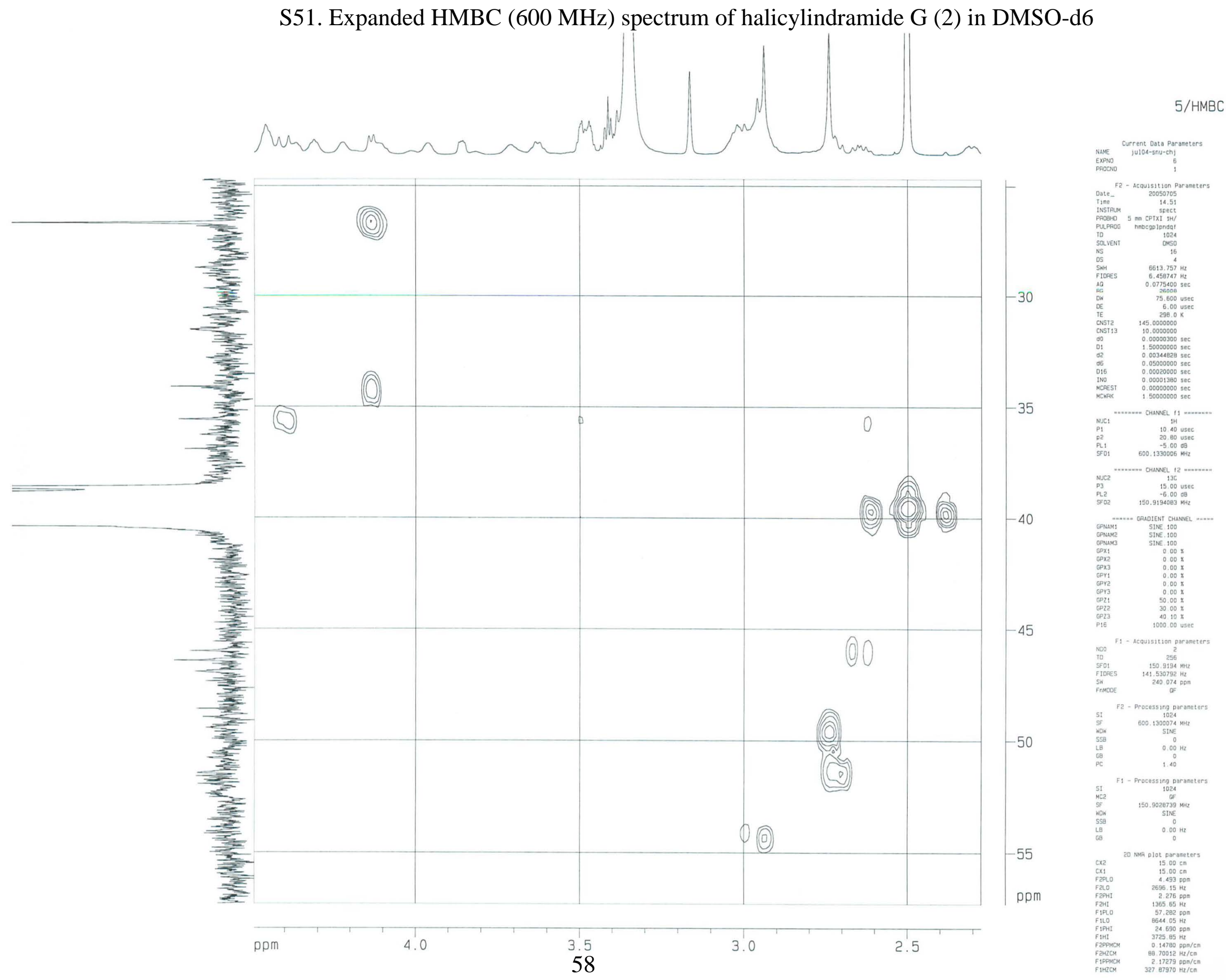


S52. HR-ESI-Tof-MS spectrum of halicylindramide H (3)

Hali H(+)-6 157 (1.969) Cm (155:158)
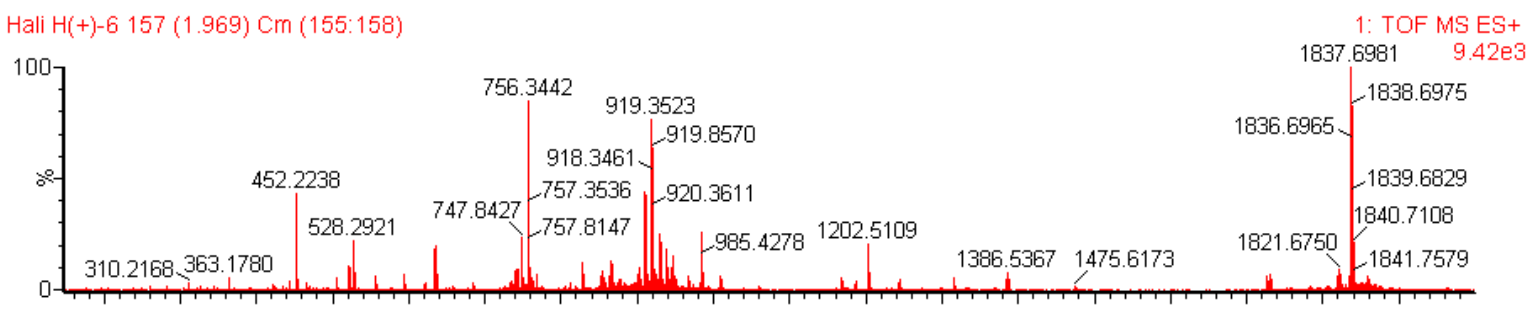

Hali H(-)-1 157 (1.965) Cm (155:158)
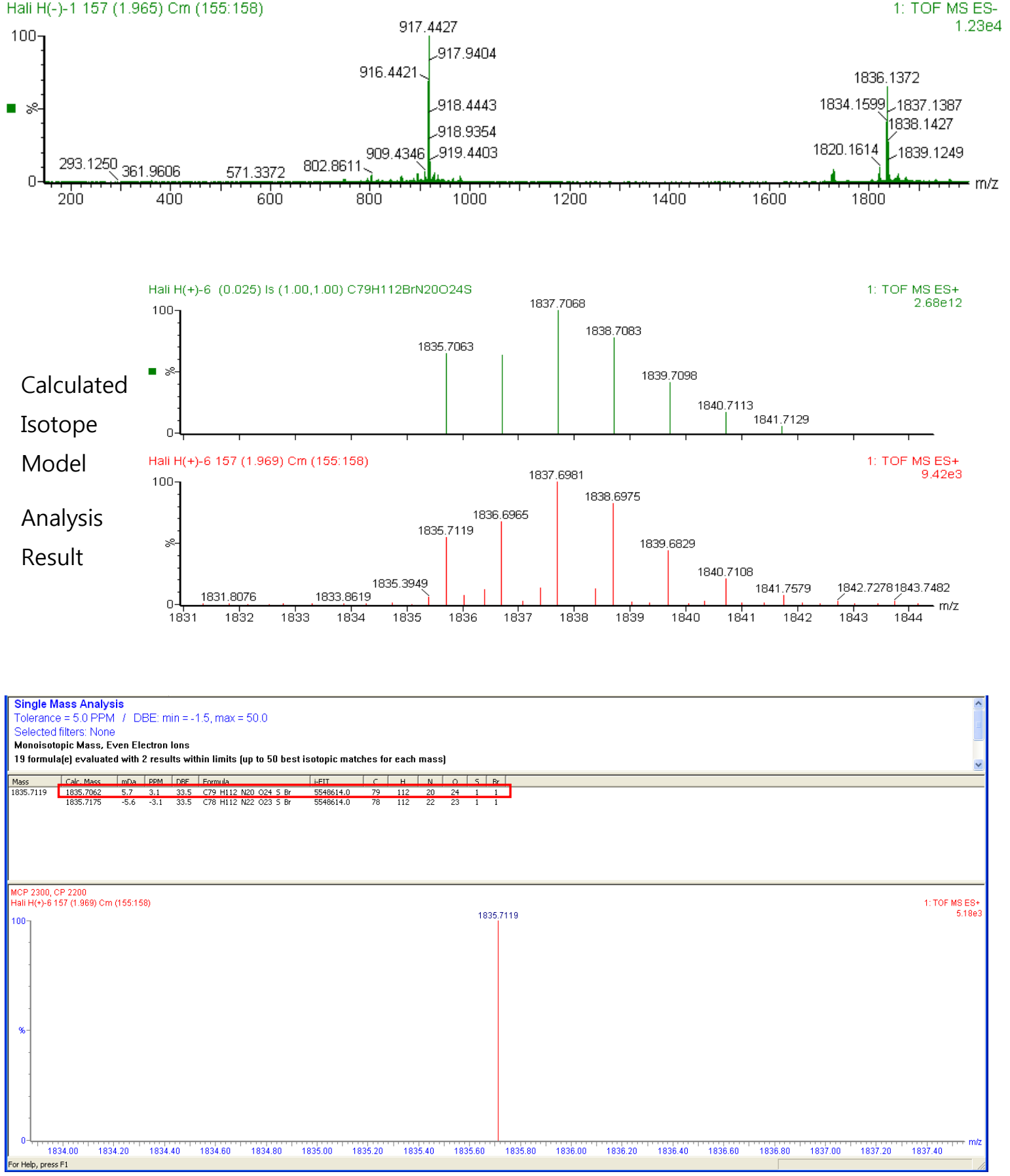
S53. 1H NMR (600 MHz) spectrum of halicylindramide H (3) in DMSO-d6

$5-1 / 1 H$

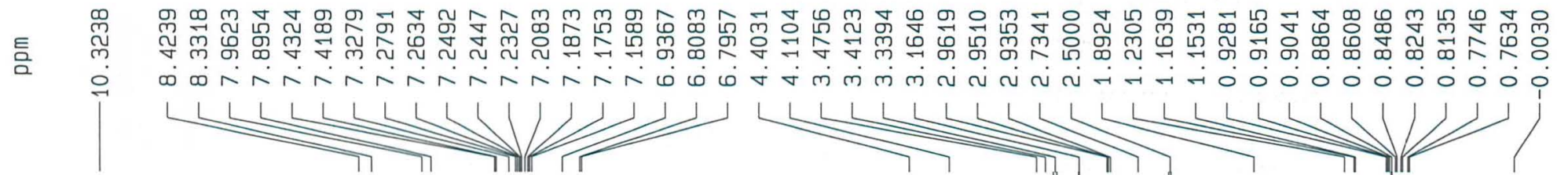

Current Data Parameters

EXPNO

PROCNO

11

F2 - Acquisition Parameters

Date_

Dime- 20050704

$\begin{array}{ll}\text { INSTRUM } & 16.09 \\ \text { spect }\end{array}$

PROBHD $5 \mathrm{~mm}$ CPTXI $1 \mathrm{H} /$

PULPROG Z $\quad$ Zg30

TD 65536

SOLVENT

NS

DS

FIDRES

$A Q$

$\mathrm{RG}$

DW

TE

D1

MCRES

8
0

$8741.259 \mathrm{~Hz}$

$0.133381 \mathrm{~Hz}$

$3.7487664 \mathrm{sec}$

28.5

57.200 usec

6.00 use

1. $00000000 \mathrm{sec}$

$0.00000000 \mathrm{sec}$

$0.01500000 \mathrm{sec}$

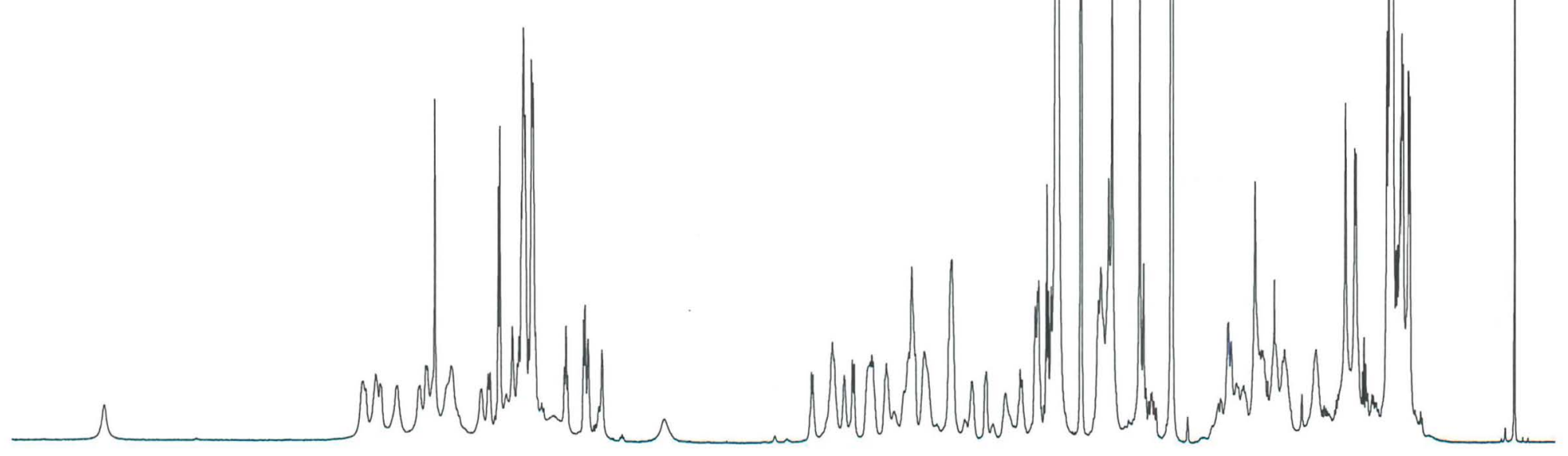

$===== \pm==$ CHANNEL $f 1$ ="s==s=

NUC1 $1 \mathrm{H}$

P1 10.30 usec

SF01 $\quad 600.1336696 \mathrm{MHz}$

F2 - Processing parameters

SI 32768

SF $\quad 600.1300071 \mathrm{MHz}$

WDW EM

$\begin{array}{lc}\text { SSB } & 0 \\ \text { LB } & 0.30 \mathrm{~Hz}\end{array}$

$\begin{array}{lr}G B & 0 \\ P C & 1.00\end{array}$

10 NMR plot parameters

CX $\quad 20.00 \mathrm{~cm}$

$\begin{array}{ll}\text { CY } & 200.00 \mathrm{~cm}\end{array}$

F1P $\quad 11.000 \mathrm{ppm}$

$\begin{array}{ll}\text { F1 } & 6601.43 \mathrm{~Hz} \\ \text { F2P } & -0.300 \mathrm{ppm}\end{array}$

F2 $-0.300 \mathrm{ppm}$

PPMCM $-180.04 \mathrm{~Hz}$

HZCM $\quad 339.07346 \mathrm{~Hz} / \mathrm{cm}$ 
S54. 13C NMR (150 MHz) spectrum of halicylindramide H (3) in DMSO-d6

$$
5-1 / C 13
$$

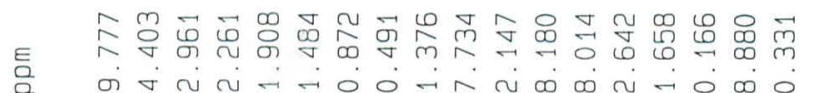
a

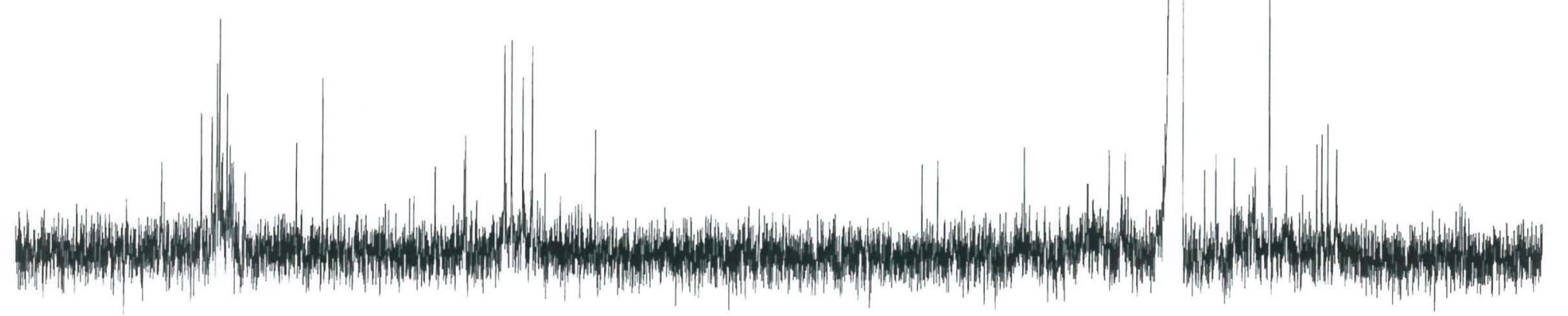

$\mathrm{ppm}$

175

150

125

100

61

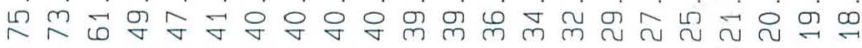

Current Data Parameters

NAME juI08-snu-ch]

PROCNO

F2 - Acquisition Parameters

Date_ 20050717

Time

INSTRUM

11 ?7

PROBHD $5 \mathrm{~mm}$ Dual 13

PULPROG $29 d c$

TD TOLVNT $\quad 65536$

NS $\quad 46080$

SWH $\quad 21097.047 \mathrm{~Hz}$

FIDRES $\quad 0.321915 \mathrm{~Hz}$

$A Q \quad 1.5532532 \mathrm{sec}$

$\begin{array}{lr}\text { AG } & 8192 \\ \text { OW } & 23.700 \text { use }\end{array}$

DE $\quad 6.00$ usec

$012.00000000 \mathrm{sec}$

$0110.03000000 \mathrm{sec}$

$\begin{array}{lr}========= & \text { CHANNEL } f 1==== \\ \text { NUC1 } & 13 \mathrm{C} \\ \text { P1 } & 10.60 \mathrm{useC} \\ \text { PL1 } & 0.00 \mathrm{~dB} \\ \text { SF01 } & 75.4760204 \mathrm{MHZ}\end{array}$

$===== \pm==-=$ CHANNEL $f 2=$

CPDPRG2 waltz16

NUC2 $1 \mathrm{H}$

PCPD2 100.00 usec

PL2 $\quad-5.00 \mathrm{~dB}$

$\begin{array}{lr}\text { PL12 } & 15.00 \mathrm{~dB} \\ \text { SF02 } & 300.1312005 \mathrm{MHZ}\end{array}$

F2 - Processing parameters

SI 32768

SF $\quad 75.4677190 \mathrm{MHz}$

WDW

LB $\quad 1.00 \mathrm{HZ}$

$\begin{array}{lr}\mathrm{OB} & 0 \\ \mathrm{PC} & 1.40\end{array}$

10 NMF plot parameters

CX $\quad 20.00 \mathrm{~cm}$

F1P $\quad 200.000 \mathrm{ppm}$

F1 $15093.54 \mathrm{~Hz}$

$\begin{array}{ll}\text { F2P } & -10.000 \mathrm{ppm} \\ \mathrm{FP} & -754.68 \mathrm{~Hz}\end{array}$

PPMCM $\quad 10.50000 \mathrm{ppm} / \mathrm{cm}$

$\begin{array}{lll}\mathrm{HZCM} & 792.41107 \mathrm{~Hz} / \mathrm{cm}\end{array}$ 
S55. HSQC (600 MHz) spectrum of halicylindramide H (3) in DMSO-d6

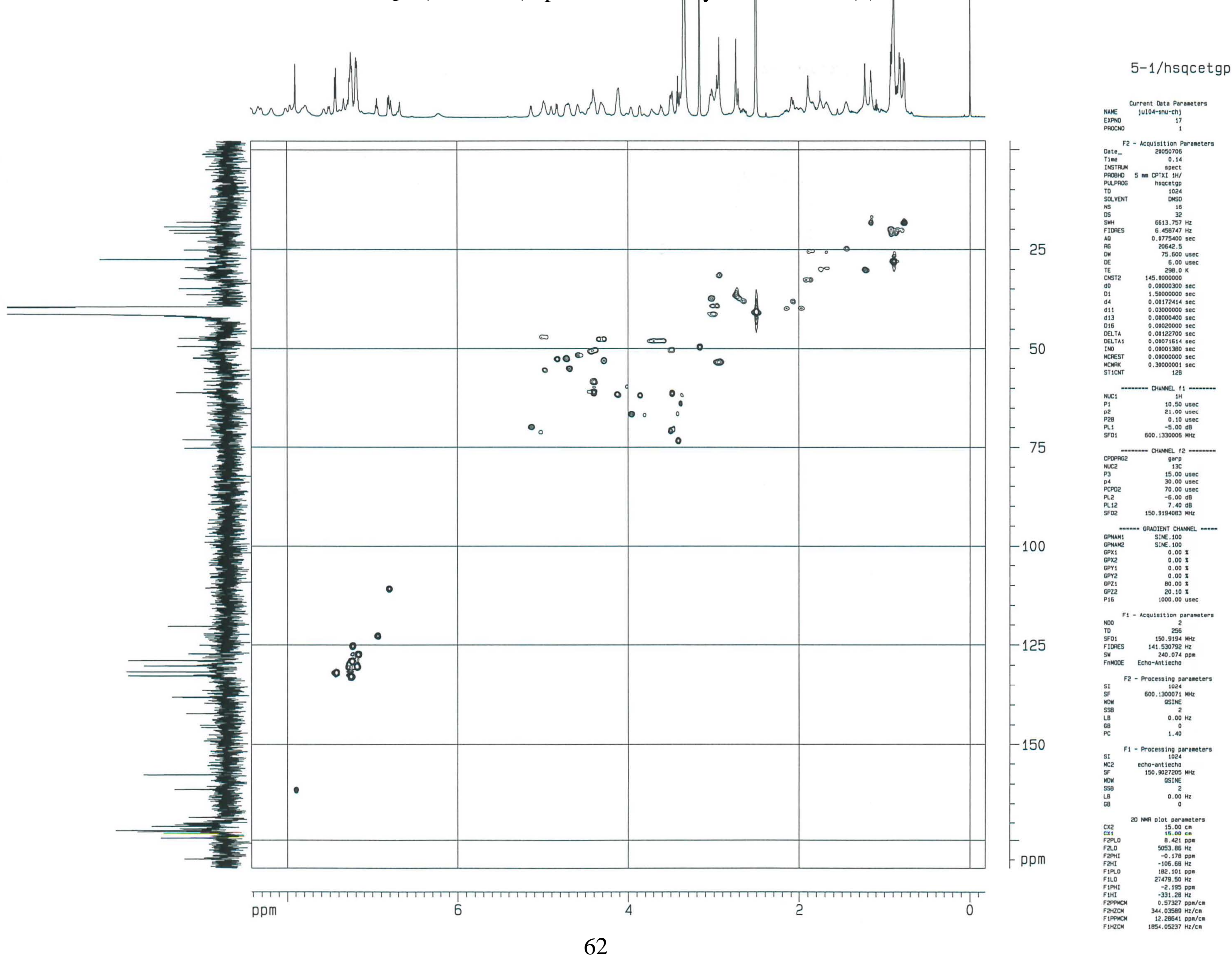


S56. COSY (600 MHz) spectrum of halicylindramide H (3) in DMSO-d6
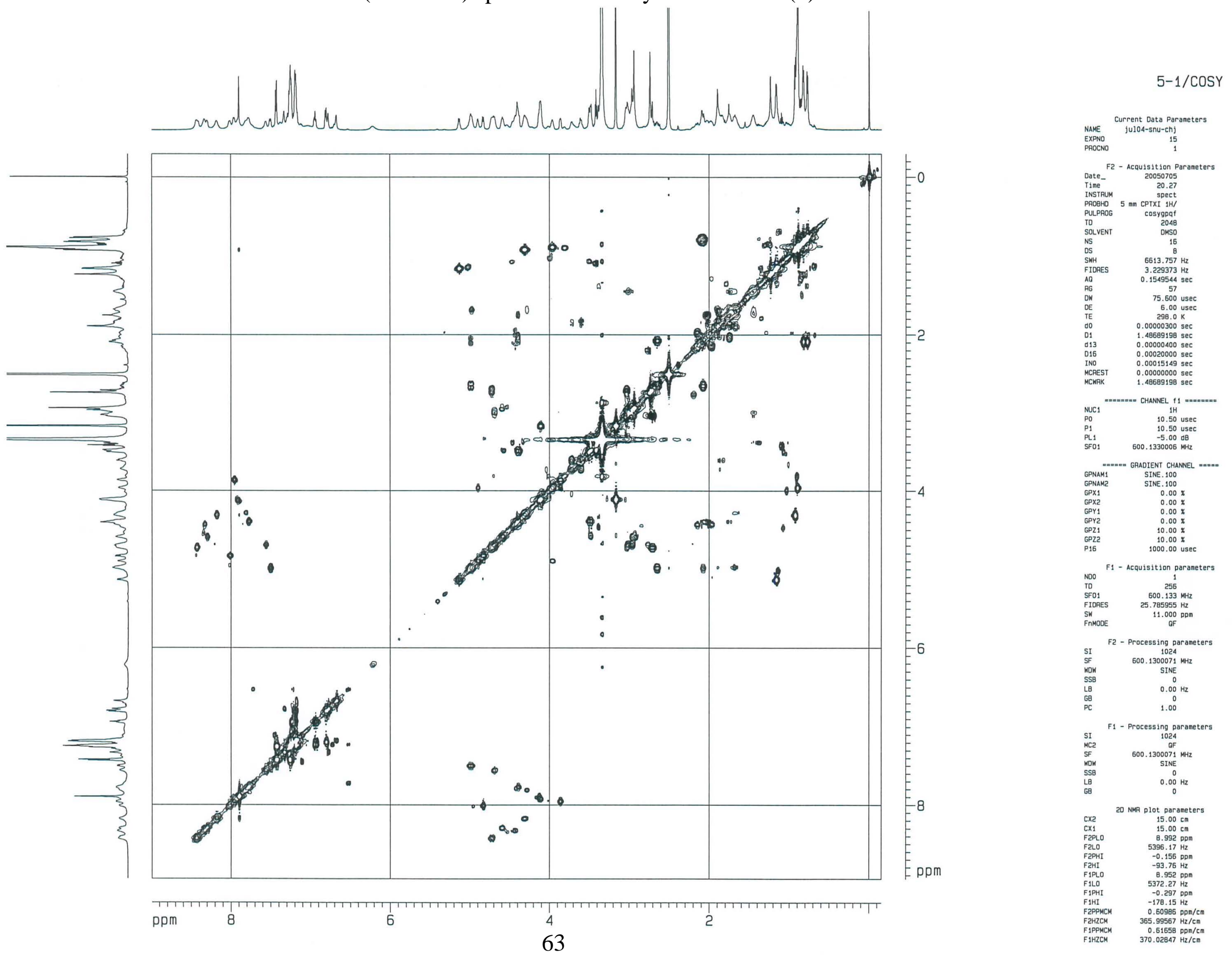
S57. Expanded COSY (600 MHz) spectrum of halicylindramide H (3) in DMSO-d6

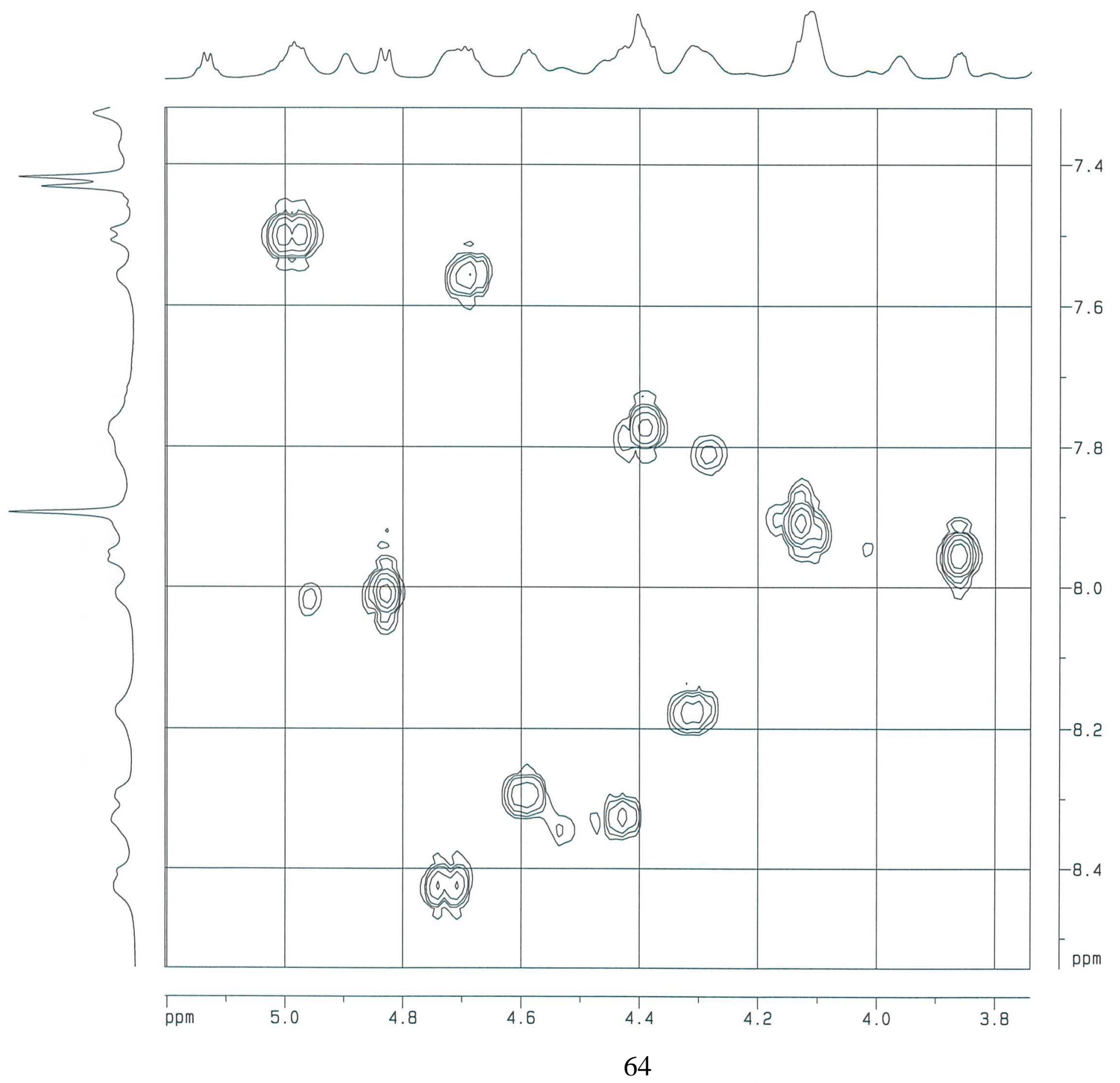

$5-1 / \operatorname{COSY}$

Current Data Parameter
NuME

NAME
EXPNO Ju104-snu-ch,
PPOCNO

F2 - Acquisition Paraneters
20050705
Date $_{-}$

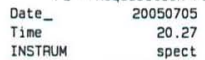

PRoOHO
PulpRoG

$\begin{array}{lr}\text { TD } & 2048 \\ \text { SOLVENT } & \text { DMSO } \\ \text { NS } & 16\end{array}$

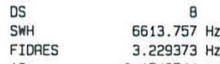

$\begin{array}{lr}\text { A } & 0.1549544 \mathrm{sec} \\ \text { AG } & 57 \\ \text { OW } & 75.600 \mathrm{us}\end{array}$

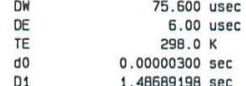

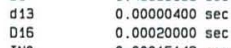

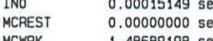

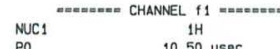

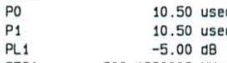

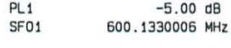

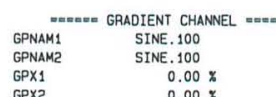

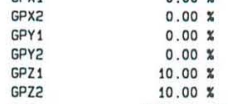

$\underset{6}{6.162}$

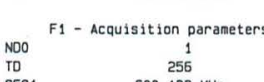

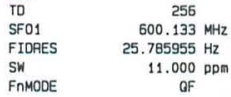

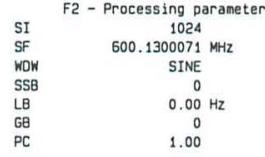

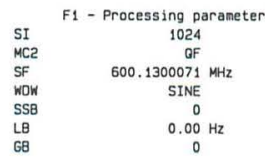

$c \times 2$
$c \times 20$
$c \times 1$

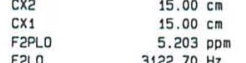

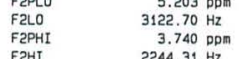

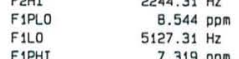

FHAI $49392.41 \mathrm{~Hz}$

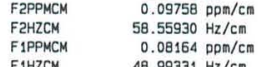




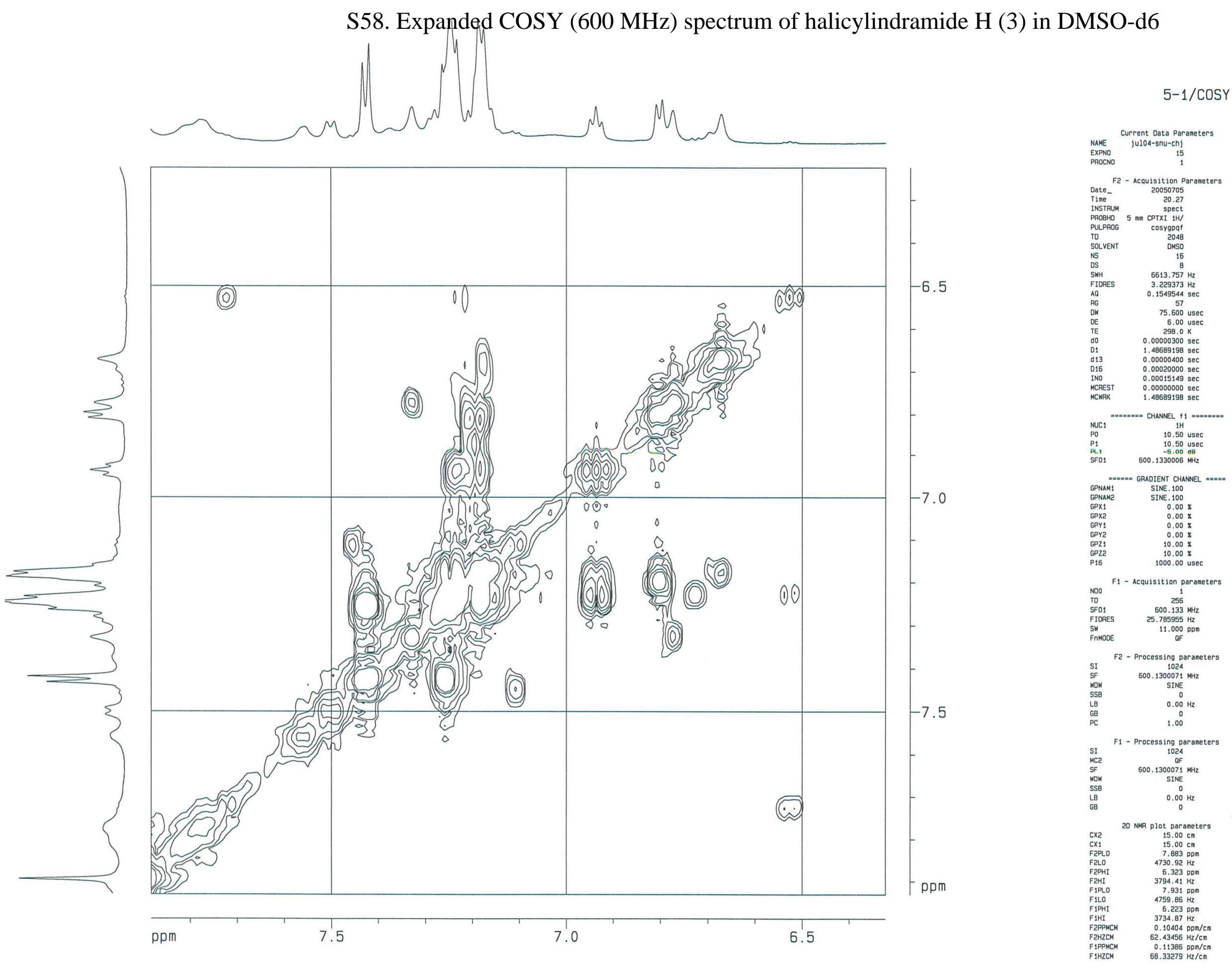


S59. Expanded COSY (600 MHz) spectrum of halicylindramide H (3) in DMSO-d6

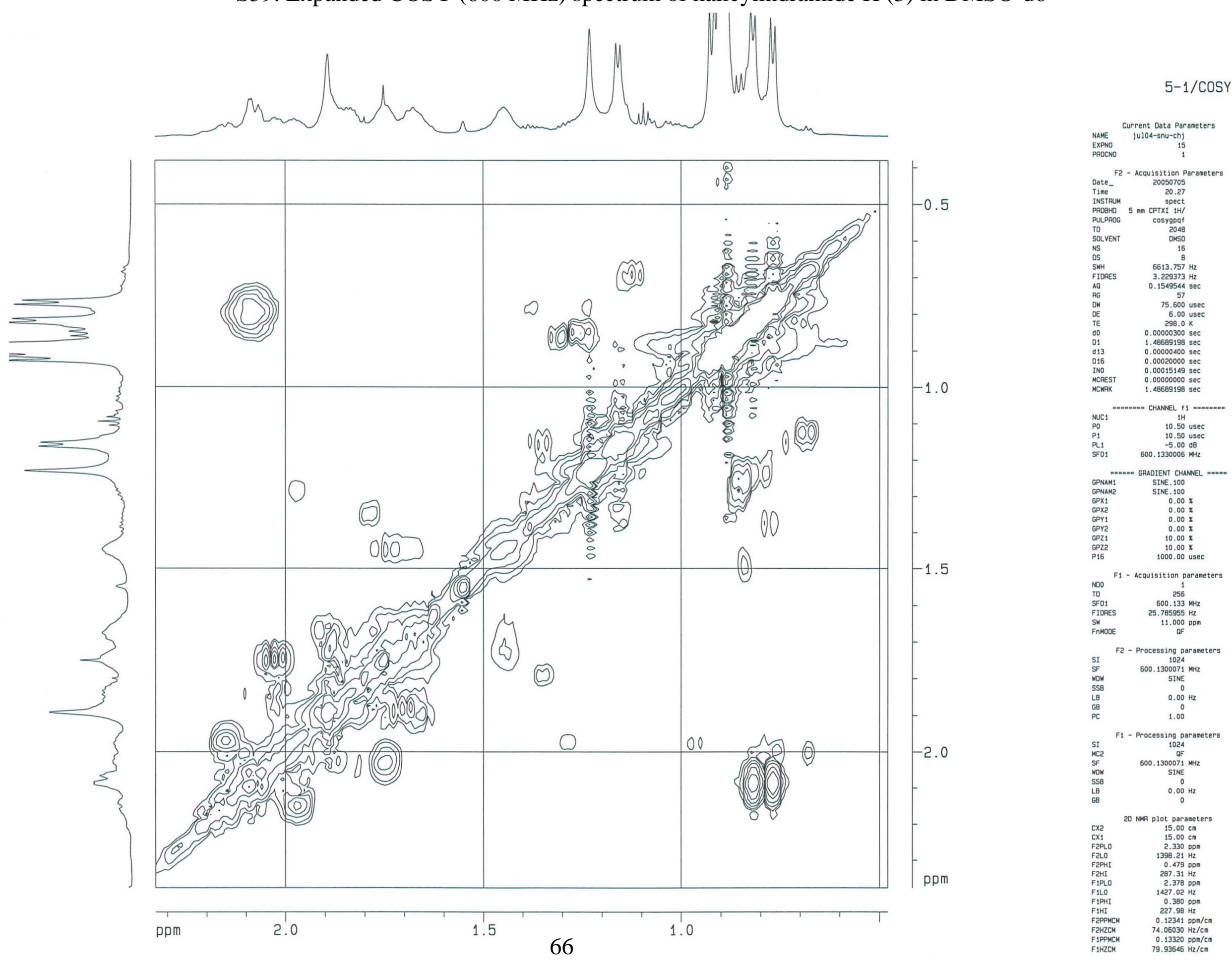


S60. Expanded COSY (600 MHz) spectrum of halicylindramide H (3) in DMSO-d6

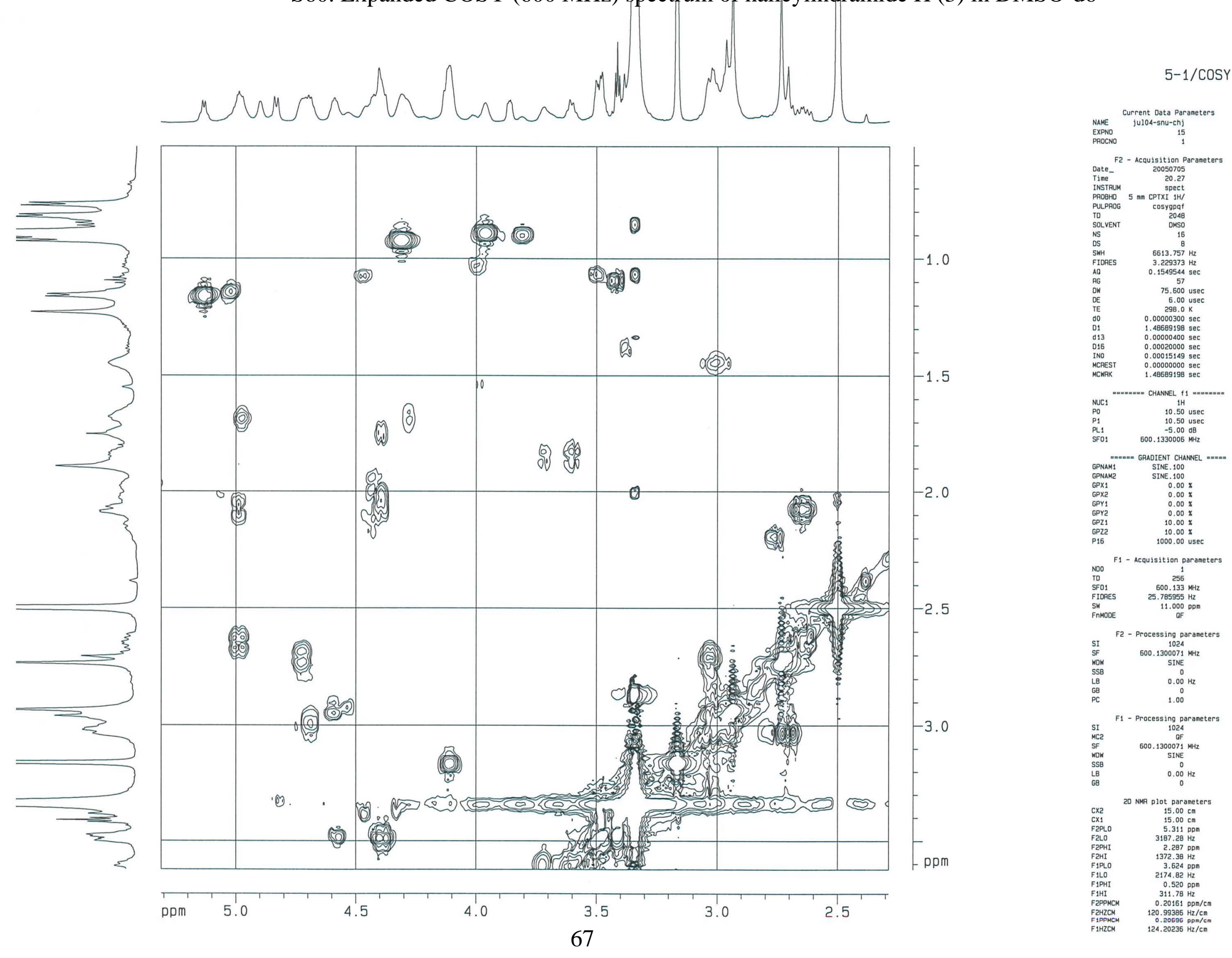


S61. TOCSY spectrum of halicylindramide H (3) in DMSO-d6

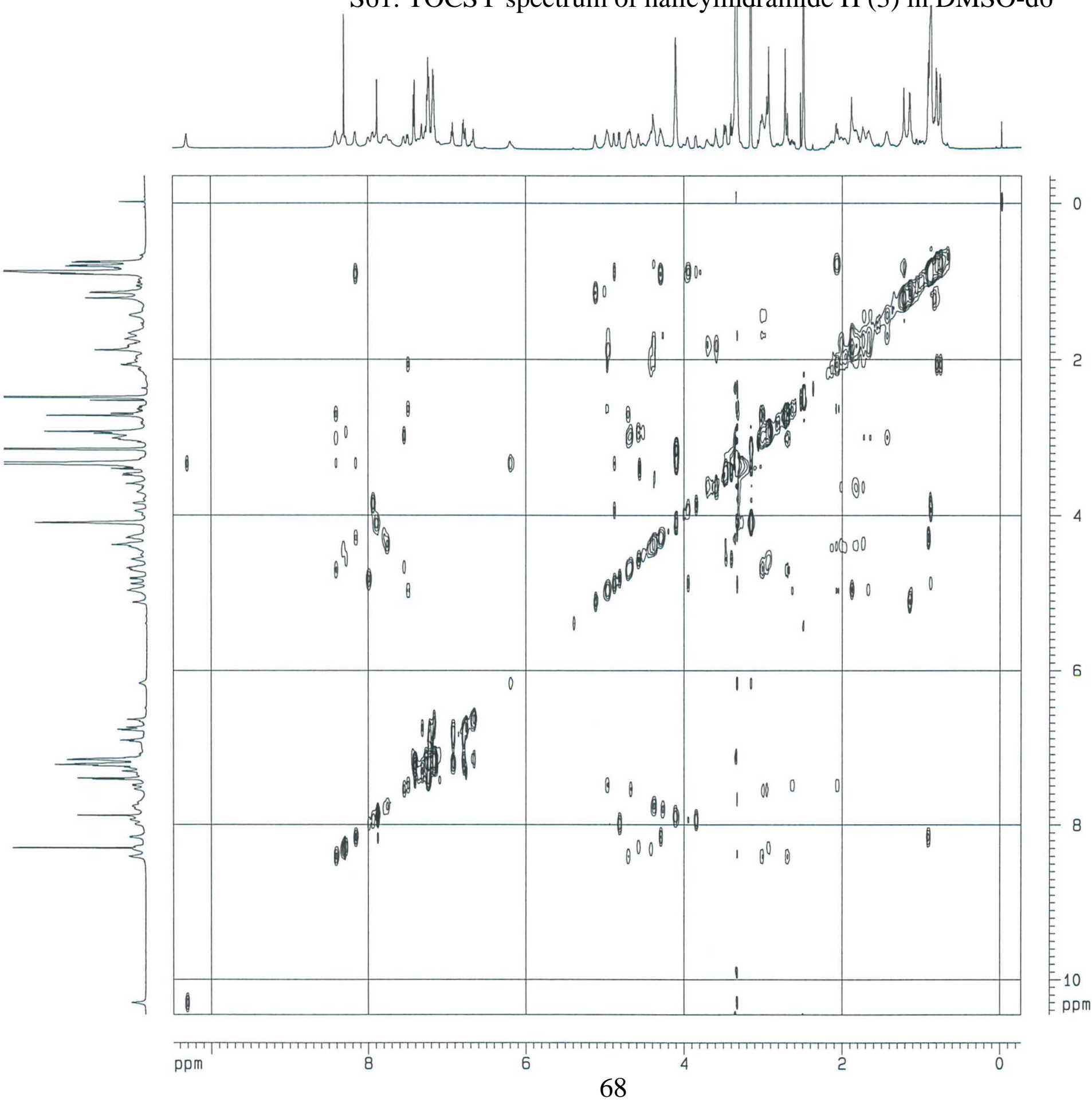

5-1/TOCSY

MAME Current Data Parameters

$\begin{array}{lr}\text { NAME } & \text { oct25-snu-chj } \\ \text { EXPNO } & 13 \\ \text { PPOCNO } & 1\end{array}$

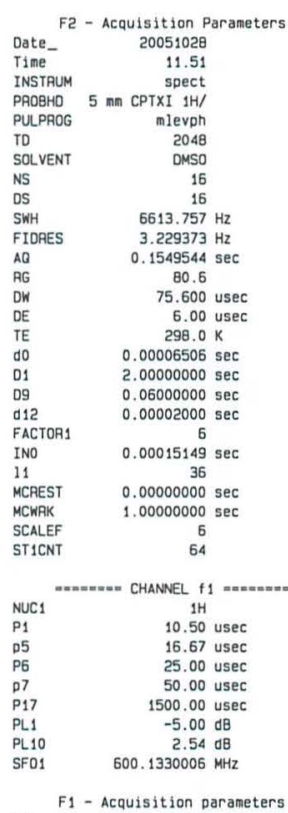

$\begin{array}{lc}\text { NOO } & 1 \\ \text { TD } & 128 \\ \text { SF01 } & 600.133 \mathrm{MHZ}\end{array}$

$\begin{array}{lr}\text { PIDRES } & 51.571911 \mathrm{~Hz} \\ \text { SM } & 11.000 \mathrm{ppm} \\ \text { FnMOOE } & \text { States-TPPI }\end{array}$

F2 - Process ing parameters

$\begin{array}{lc}\text { SI } & 1024 \\ \text { SF } & 600.1300162 \\ \text { KOH } & \text { OSINE } \\ \text { KOW } & \end{array}$

$\begin{array}{ll}\text { SSB } & 2 \\ \text { LB } & 0.00 \mathrm{HZ} \\ \text { GB } & 0 \\ \text { PC } & 1.00\end{array}$

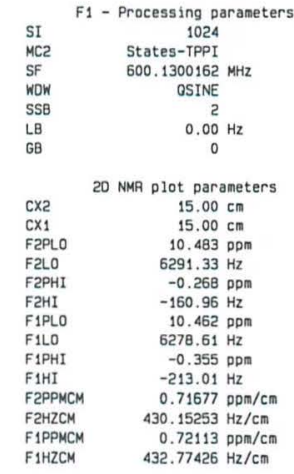


S62. Expanded TOCSY (600 MHz) spectrum of halicylindramide H (3) in DMSO-d6

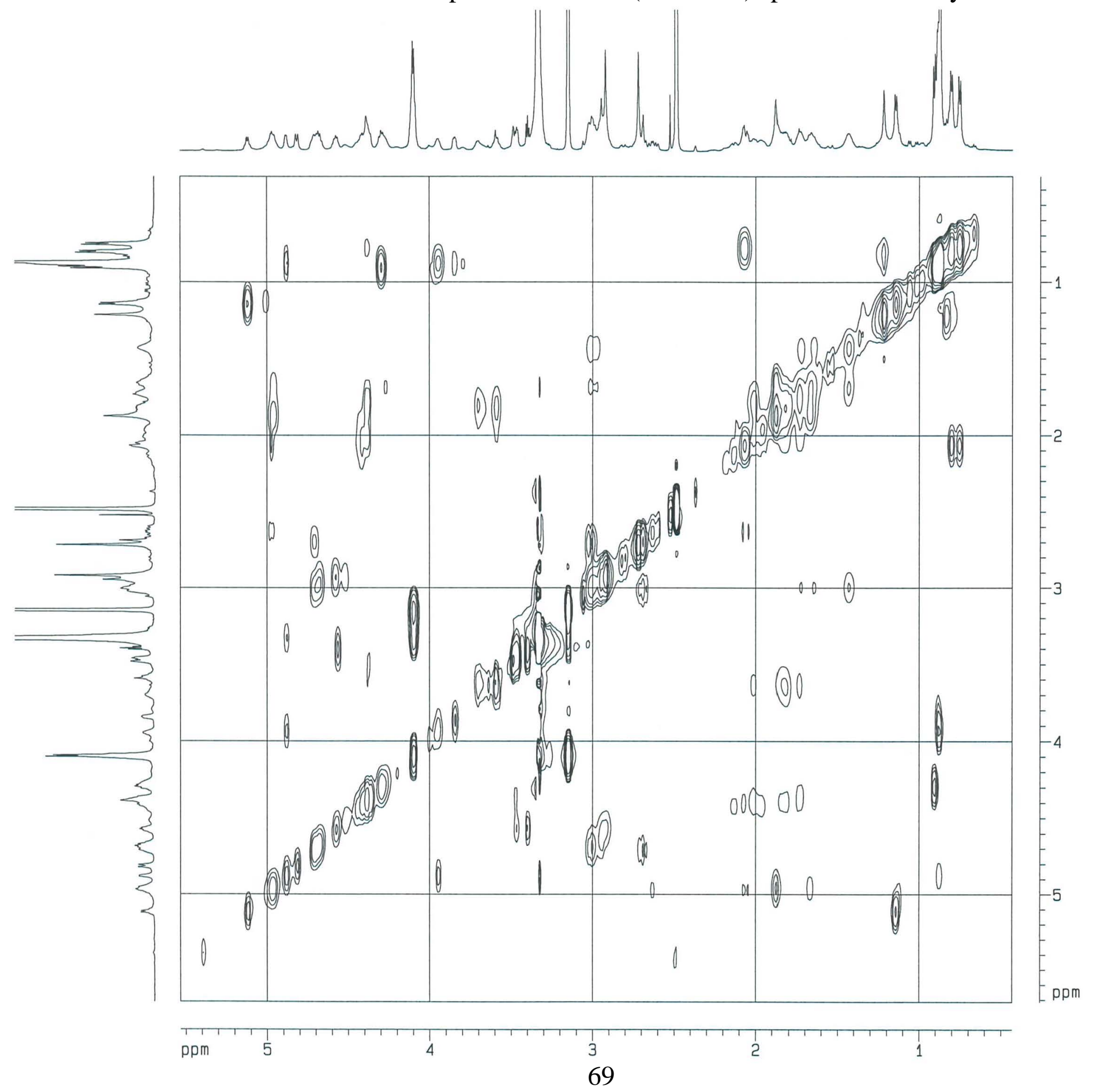

$5-1 /$ TOCSY

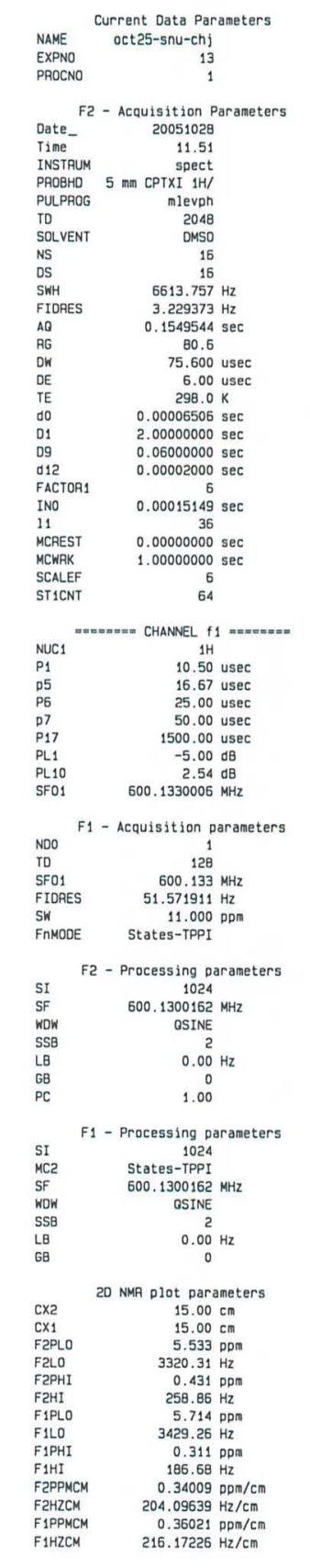


S63. Expanded TOCSY (600 MHz) spectrum of halicylindramide H (3) in DMSO-d6

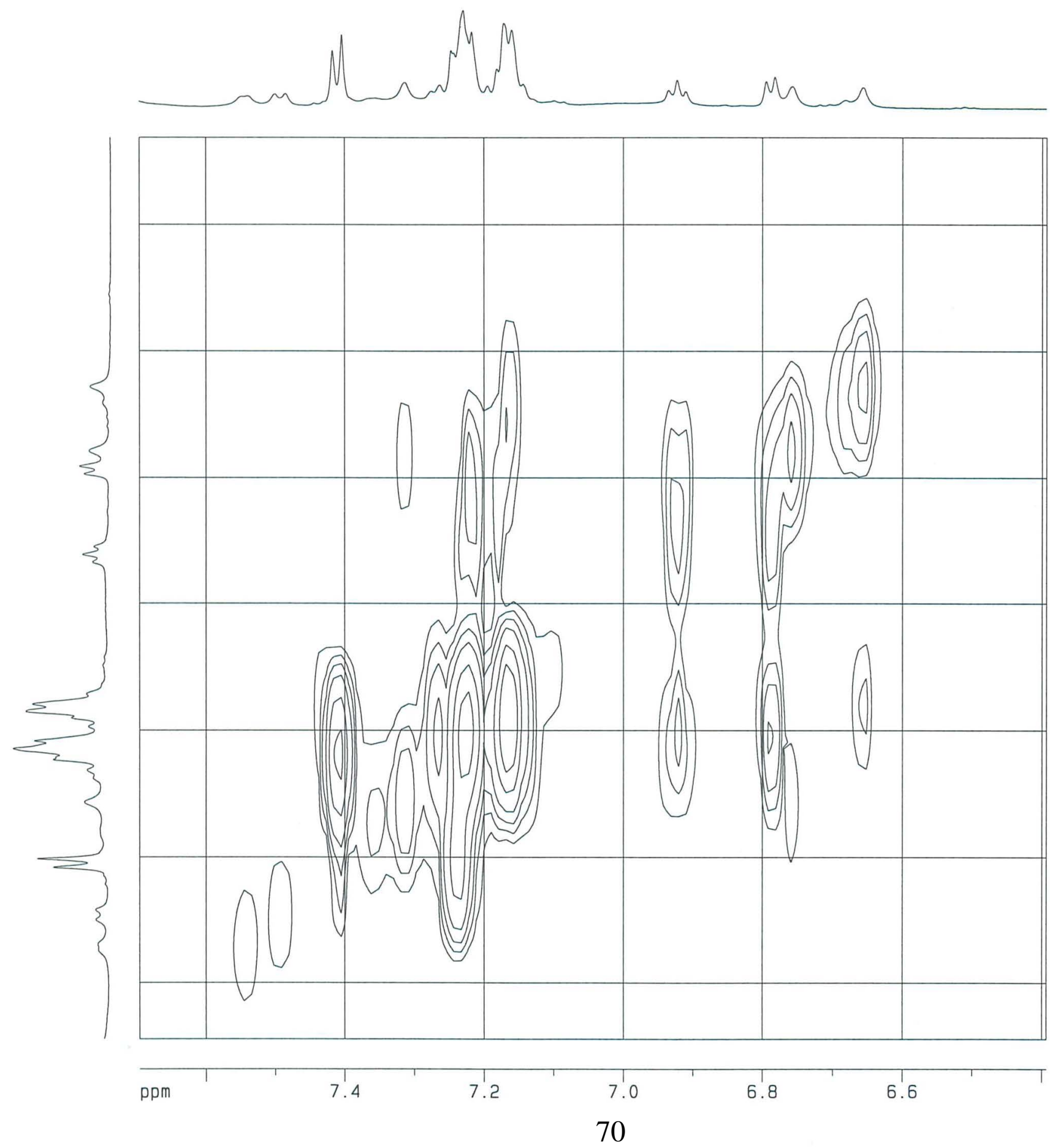

$5-1 /$ TOCSY

Nurrent Data Paramet
NAME
OCt25-snu-chj
EXPNO

PROCNO 1

$-6.4$

$-6.6$

$-6.8$

Date
Time
Time

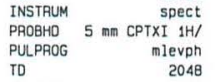

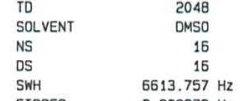

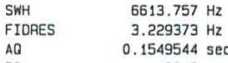

$\begin{array}{ll}\text { PG } & 0.1549544 \mathrm{sec} \\ \text { OH } & \quad 80.6 \\ \text { OE } & 75.600 \mathrm{usec} \\ \text { OE } & 6.00 \mathrm{usec} \\ \text { TE } & 298.0 \mathrm{~K}\end{array}$

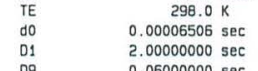

$\begin{array}{ll}0.06000000 \mathrm{sec} \\ 012 & 0.00000000 \mathrm{sec}\end{array}$

$\underset{11}{\text { INO }} \quad 0.00015149 \mathrm{sec}$

$\begin{array}{ll}\text { MCAEST } & 0.000000000 \mathrm{sec} \\ \text { MCRAK } & 1.00000000 \mathrm{sec}\end{array}$

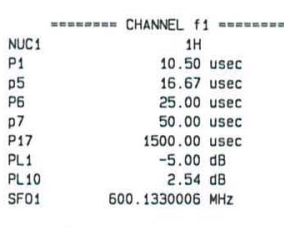

F1 - Acquisition parameters

$\begin{array}{lr}\text { NoO } & 1 \\ \text { TD } & 128 \\ \text { SF01 } & 600.139\end{array}$

$\begin{array}{lr}\text { FIDRES } & 51.571911 \mathrm{~Hz} \\ \text { SW } & 11.000 \mathrm{ppm} \\ \text { FNMODE } & \text { States-TPPI }\end{array}$

$\begin{array}{lc}\text { FI } & \text { F2 - Processsing par aneters } \\ \text { SF } & 1024 \\ \text { SF } & 600.1300202 \mathrm{MHz}\end{array}$

$\begin{array}{ll}\text { KOW } & \text { OSTNE } \\ \text { SSB } & 0 \\ \text { LE } & 0.00 \mathrm{~Hz} \\ 68 & 0 \\ \text { GC } & 1.00\end{array}$

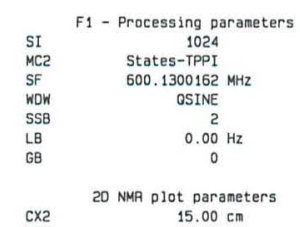

$-7.6$

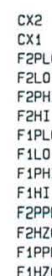

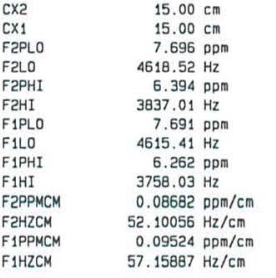




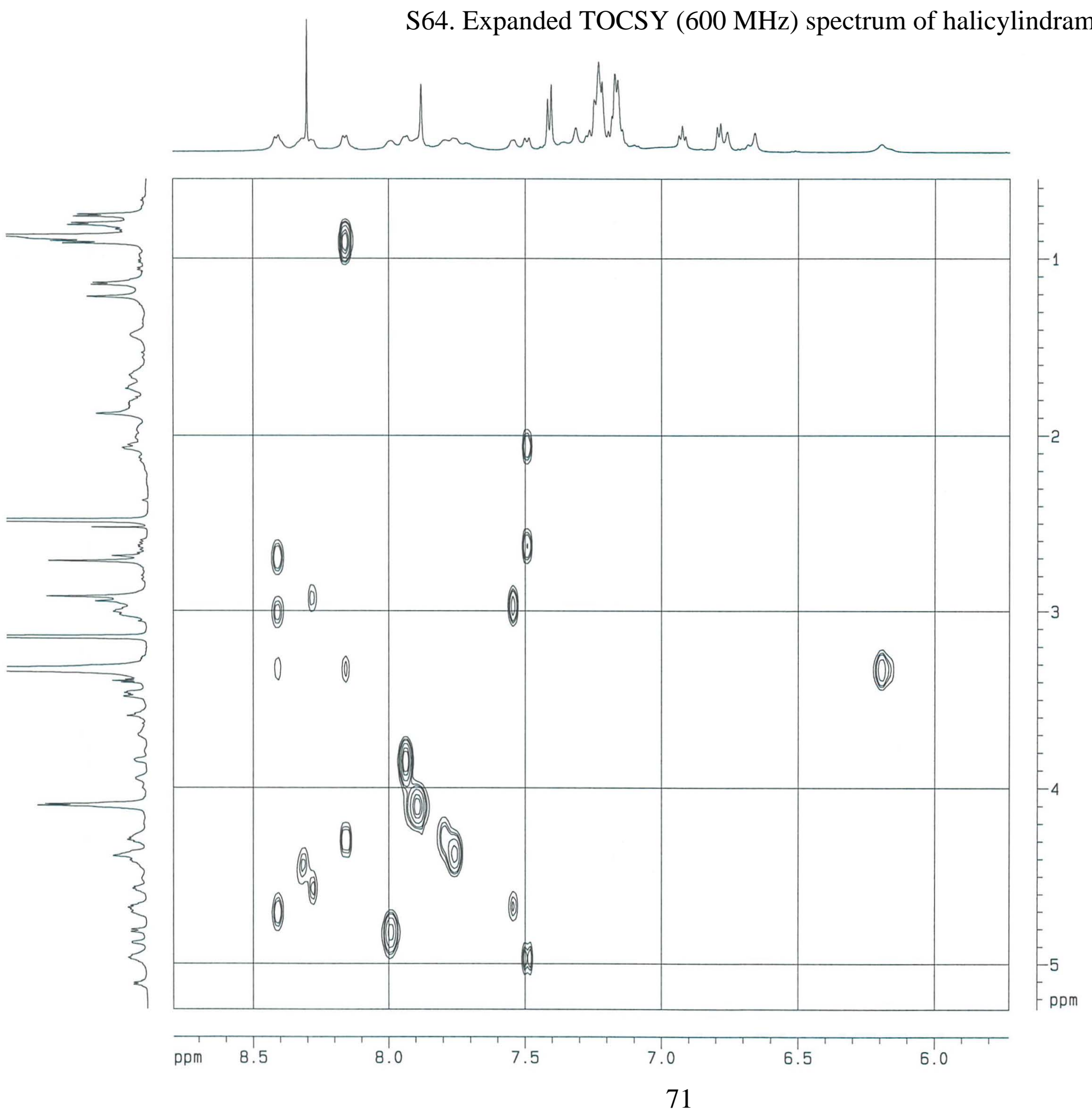

5-1/TOCSY

NaMe Current Data Parameters

NAME
EPNO
PROCNO

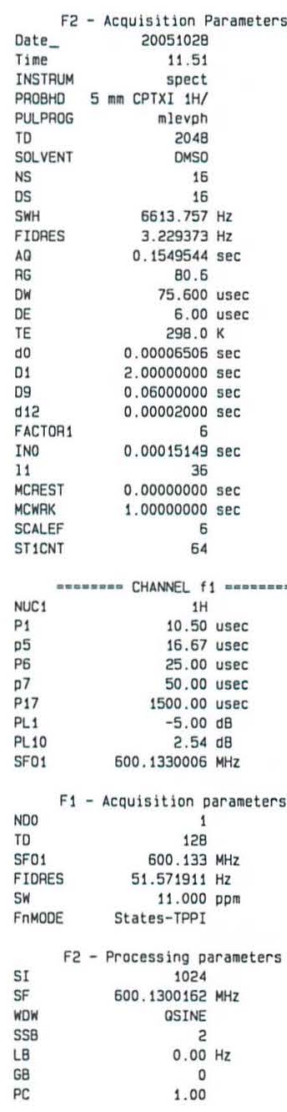

SI
SI - Processing parameters
MC2
States-TPor

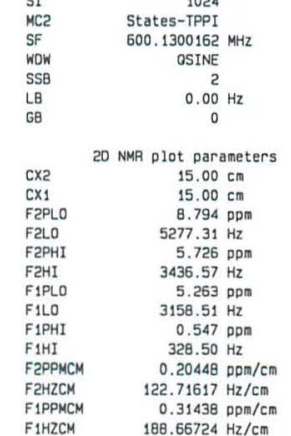


S65. NOESY (600 MHz) spectrum of halicylindramide H (3) in DMSO-d6
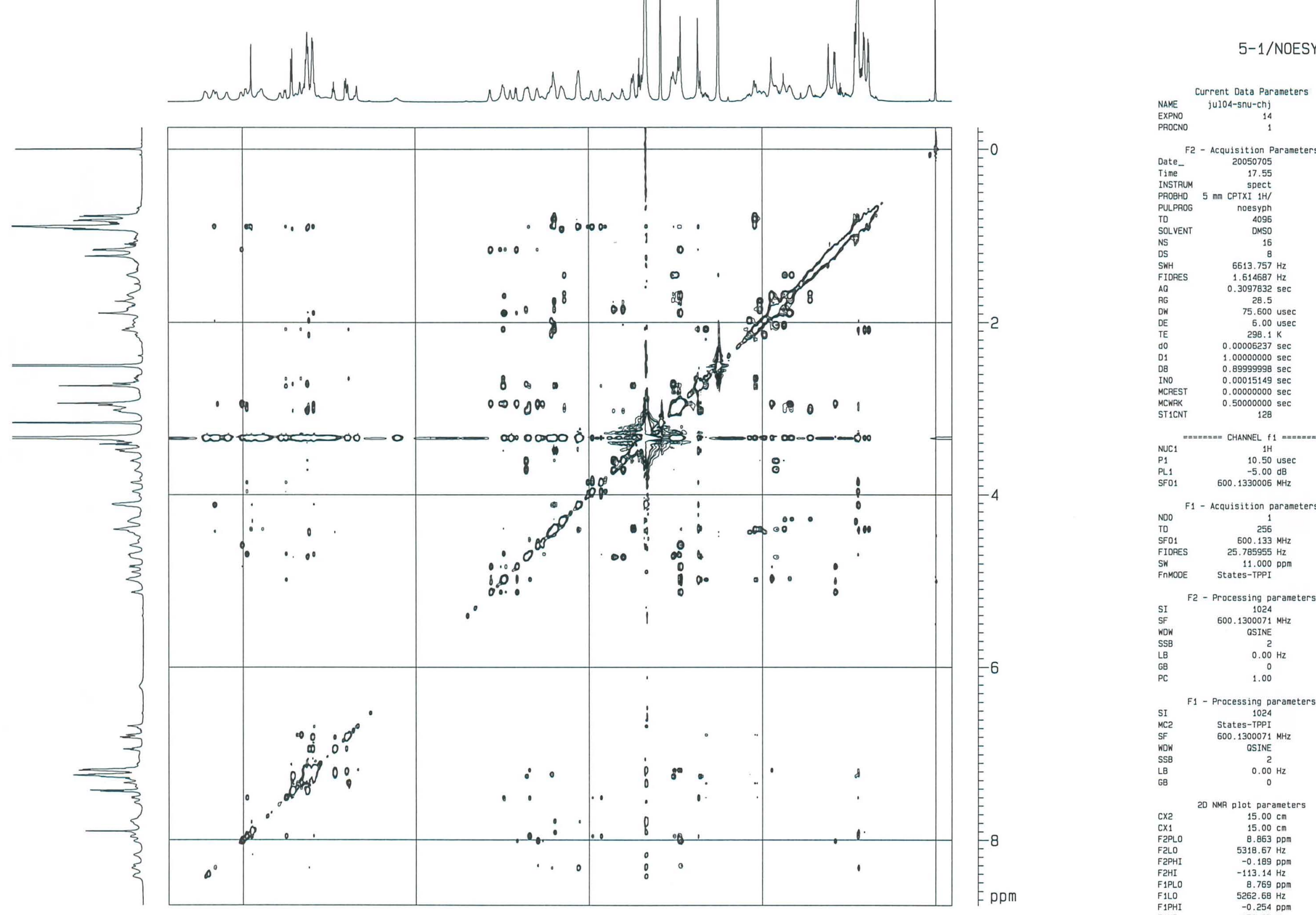

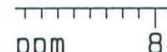

6

2

0 
S66. Expanded NOESY (600 MHz) spectrum of halicylindramide H (3) in DMSO-d6

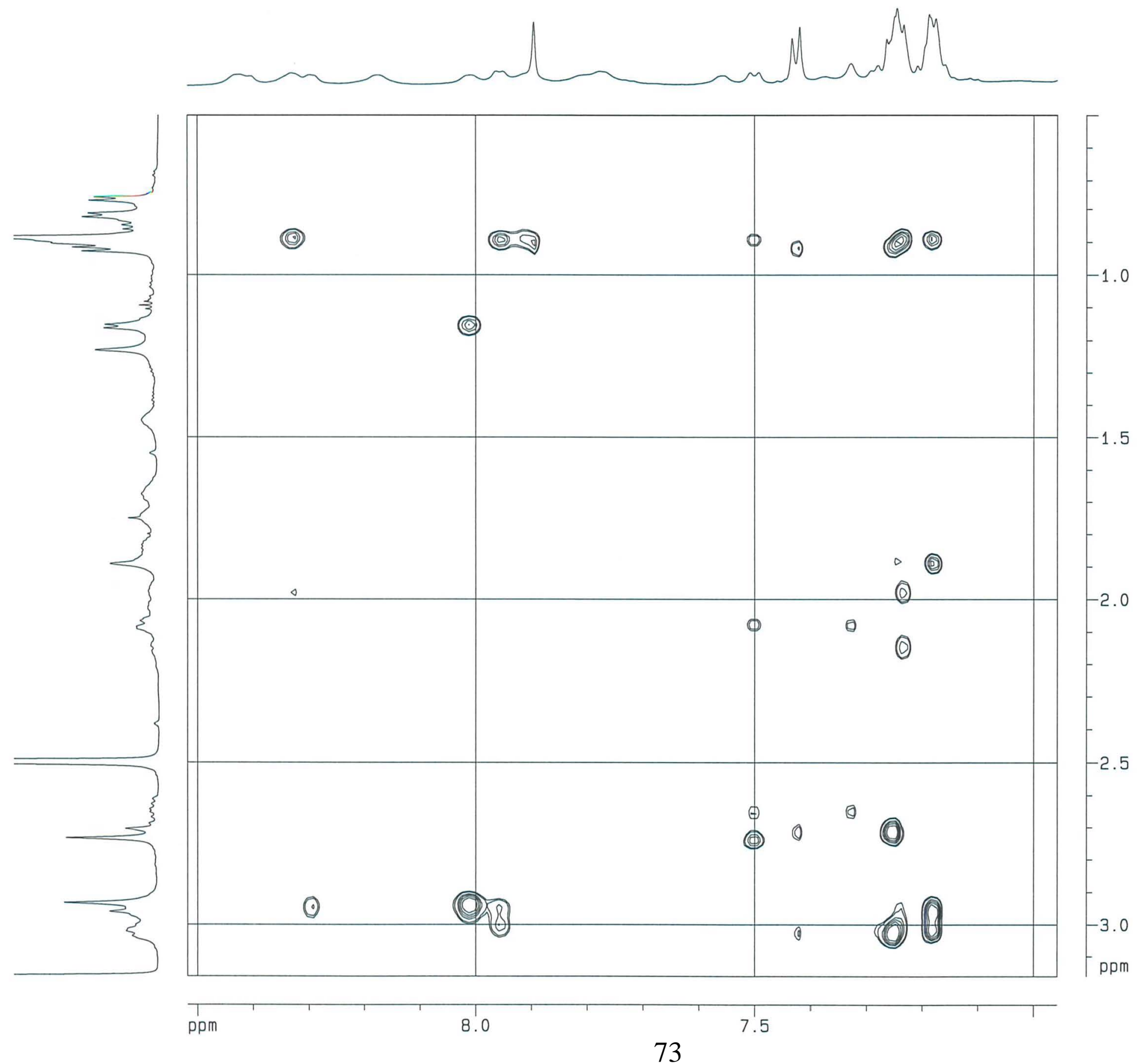

$5-1 /$ NOESY

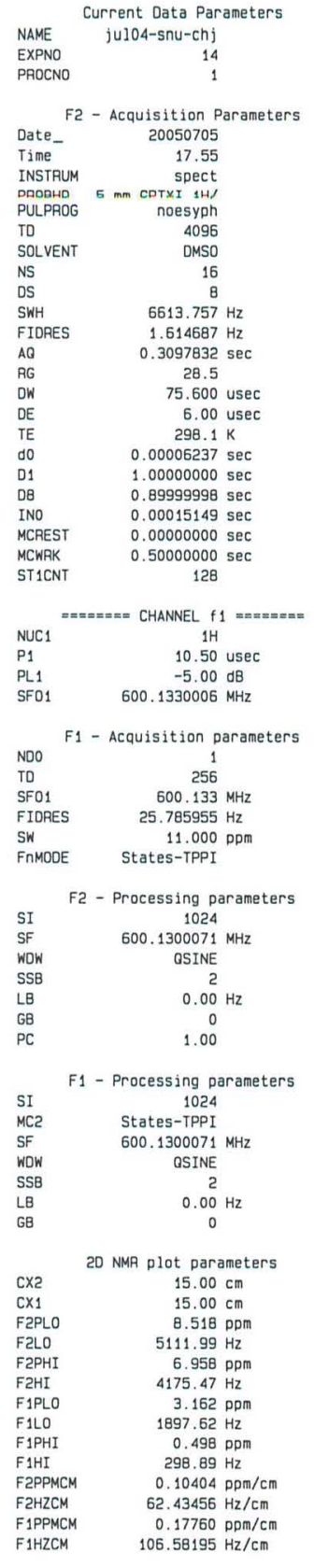




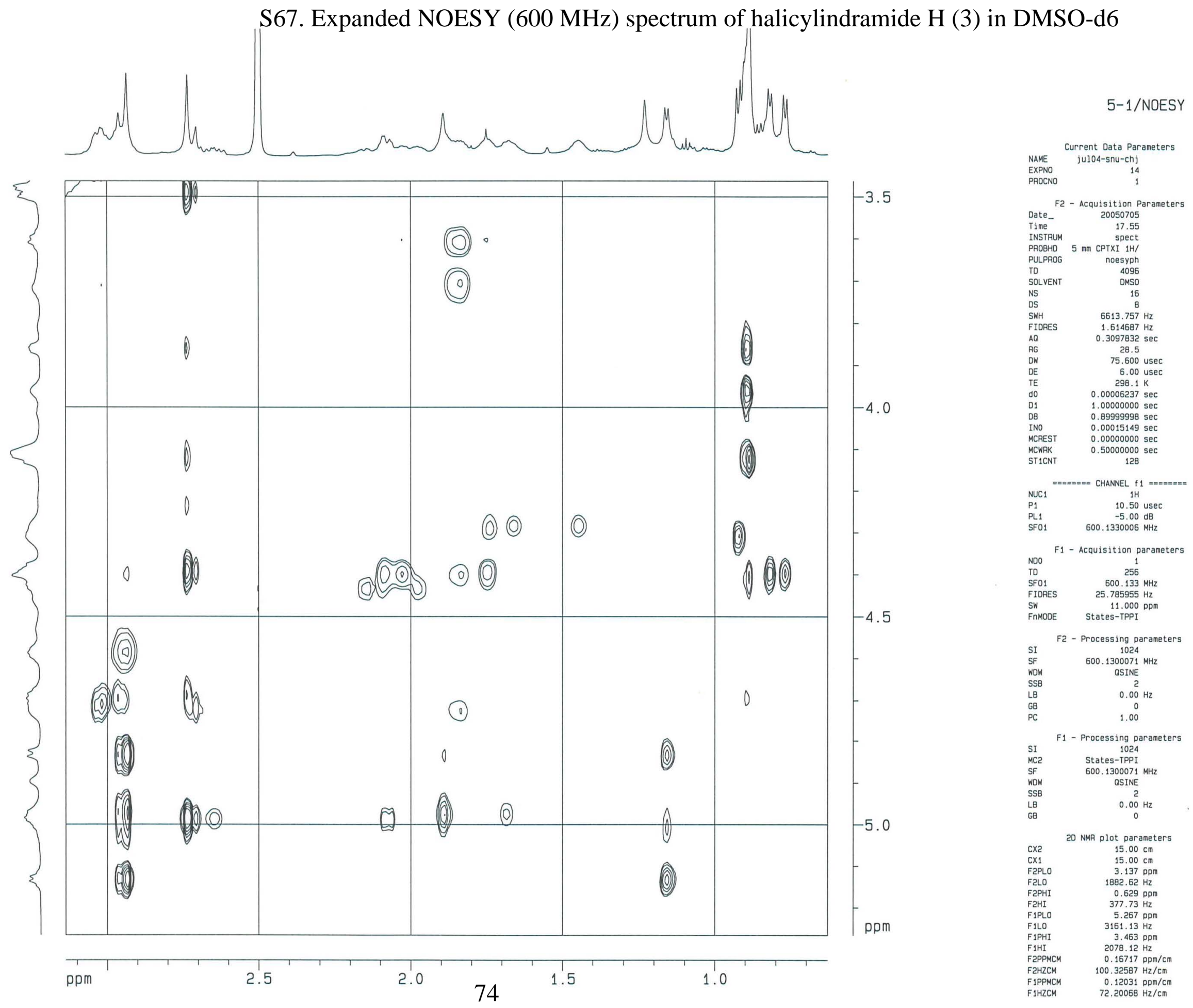


S68. Expanded NOESY (600 MHz) spectrum of halicylindramide H (3) in DMSO-d6
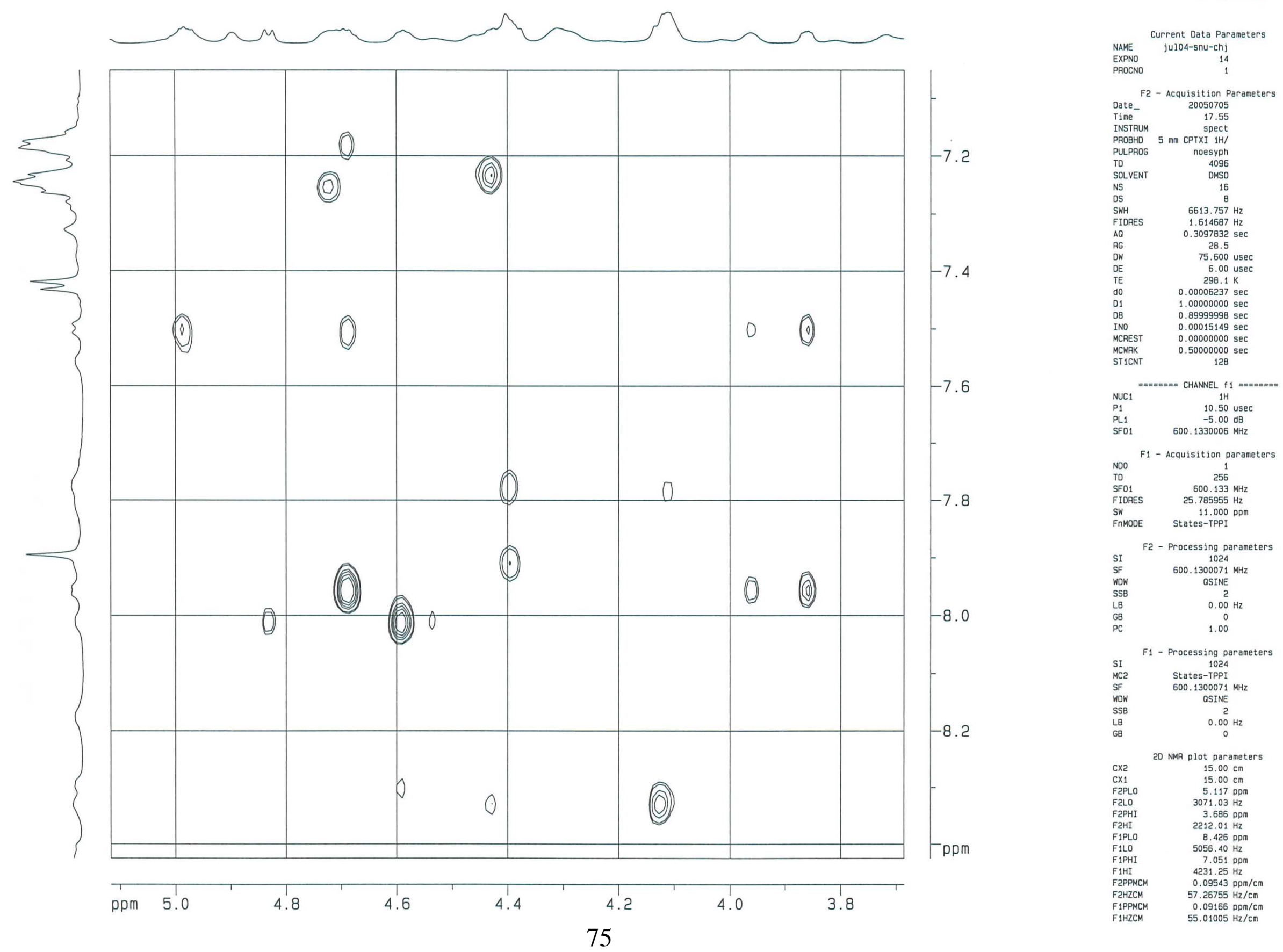
S69. Expanded NOESY (600 MHz) spectrum of halicylindramide H (3) in DMSO-d6

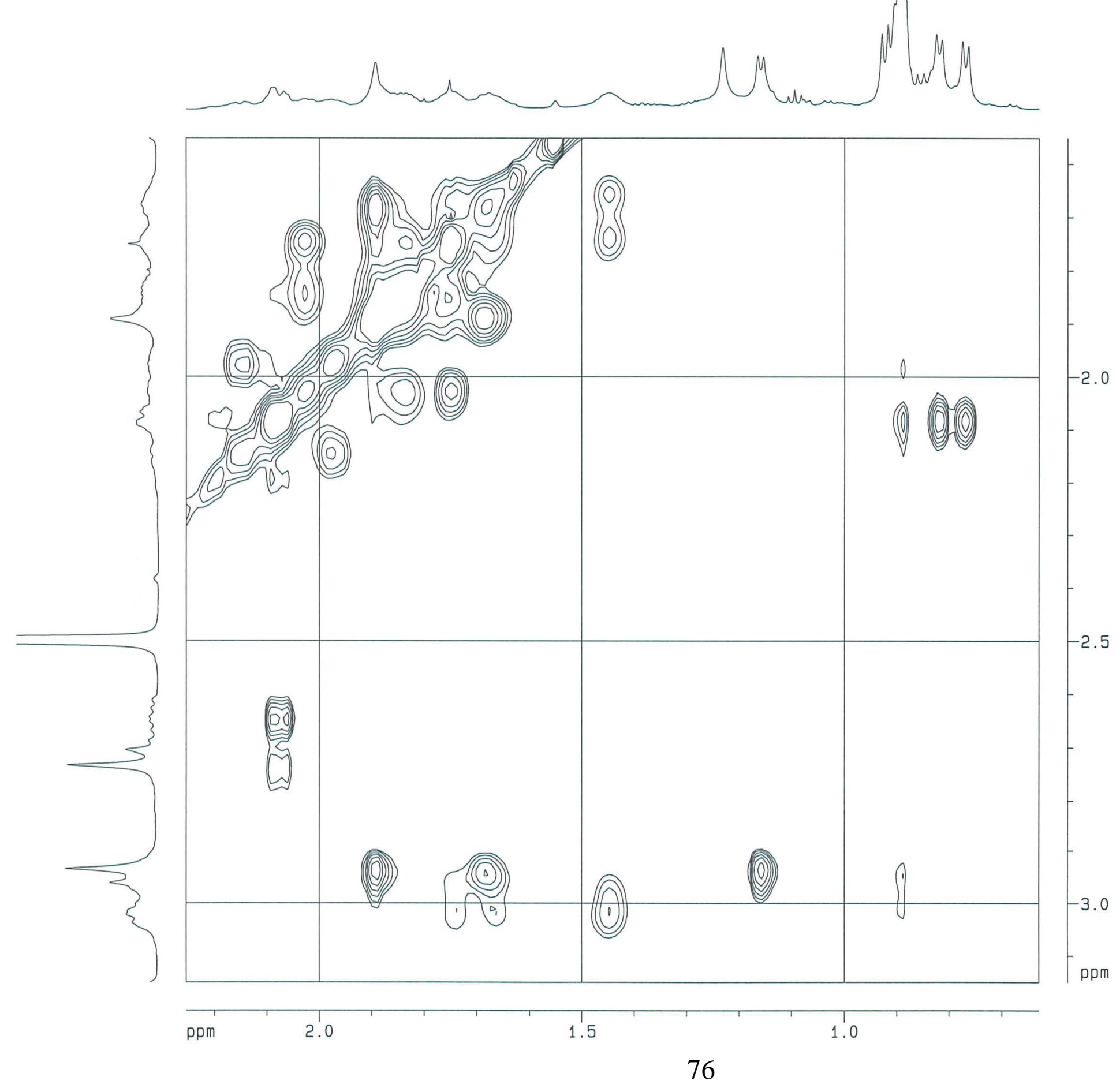

$5-1 /$ NOESY

$\begin{array}{ll}\text { NAME } & \text { ju104-SnU-chj } \\ \text { EPPNO } & 14 \\ \text { PROCNO } & 1\end{array}$

F2 - Acquisition Parameters
20050705

$\begin{array}{lr}\text { Date } & 20050705 \\ \text { Time } & 17.55 \\ & \end{array}$

INSTRUM
PROBHD $5 \mathrm{~mm}$ CPTXI $1 \mathrm{H} /$

$\begin{array}{lr}\text { PULPAOG } & \text { nOesyph } \\ \text { TD } & 4096 \\ \text { SOLVENT } & \text { DMSO }\end{array}$

$\begin{array}{lc}\text { NS } & 16 \\ \text { DS } & 8 \\ \text { SWH } & 6613.757 \mathrm{~Hz}\end{array}$

FIDRES $1.614687 \mathrm{~Hz}$

$\begin{array}{lr}A Q & 0.3097832 \\ A G & 28.5 \\ A G & 75.600 \text { us } \\ \text { DW } & 6.00\end{array}$

$\begin{array}{lrl}\mathrm{OW} & 28.5 \mathrm{usec} \\ \mathrm{DE} & 6.00 \mathrm{usec} \\ \mathrm{DE} & 298.1 \mathrm{~K}\end{array}$

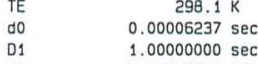

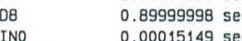

$\begin{array}{ll}\text { MCREST } & 0.00000000 \mathrm{sec} \\ \text { MCWRK } & 0.50000000 \mathrm{sec}\end{array}$

NUC1 $=== \pm==$ CHANNEL $f 1==31$.

$\begin{array}{ll}\text { NuC1 } & 1 \mathrm{H} \\ \text { P1 } & 10.50 \text { usec } \\ \text { PL1 } & -5.00 \mathrm{~dB}\end{array}$

$-5.00 \mathrm{~dB}$
PFO1

F1 - Acquisition parameters

$\begin{array}{lr}\text { No } & 1 \\ \text { TD } & 256 \\ \text { SF01 } & 500.133\end{array}$

$\begin{array}{lr}\text { FIDRES } & 65.785955 \mathrm{~Hz} \\ \text { SW } & 11.000 \mathrm{pP}\end{array}$

(1)

$\begin{array}{lc} & \text { F2 - Processing parameters } \\ \text { SI } & \text { 1024 } \\ \text { SF } & 600.1300071 \mathrm{MHz} \\ \text { SF } & \text { OSINE } \\ \text { WDH } & 0 \\ \text { SSB } & 0.00 \mathrm{~Hz} \\ \text { LB } & 0 \\ \text { GB } & 1.00 \\ \text { PC } & 1.00\end{array}$

F1 - Processing parameters

$\begin{array}{lc} & \text { F1 - Processing par } \\ \text { SI } & 1024 \\ \text { MC2 } & \text { States-TPPI }\end{array}$

$\begin{array}{lc}\text { MC2 } & \text { States-TPPI } \\ \text { SF } & 600.130071 \\ \text { WOH } & \text { OSTNE } \\ \text { SSB } & \text { SHE } \\ \text { LB } & \text { L }\end{array}$

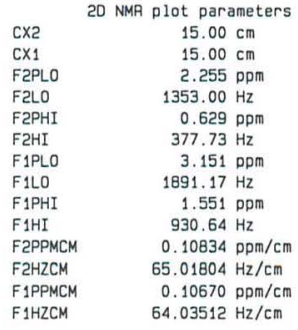


S70. Expanded NOESY (600 MHz) spectrum of halicylindramide H (3) in DMSO-d6

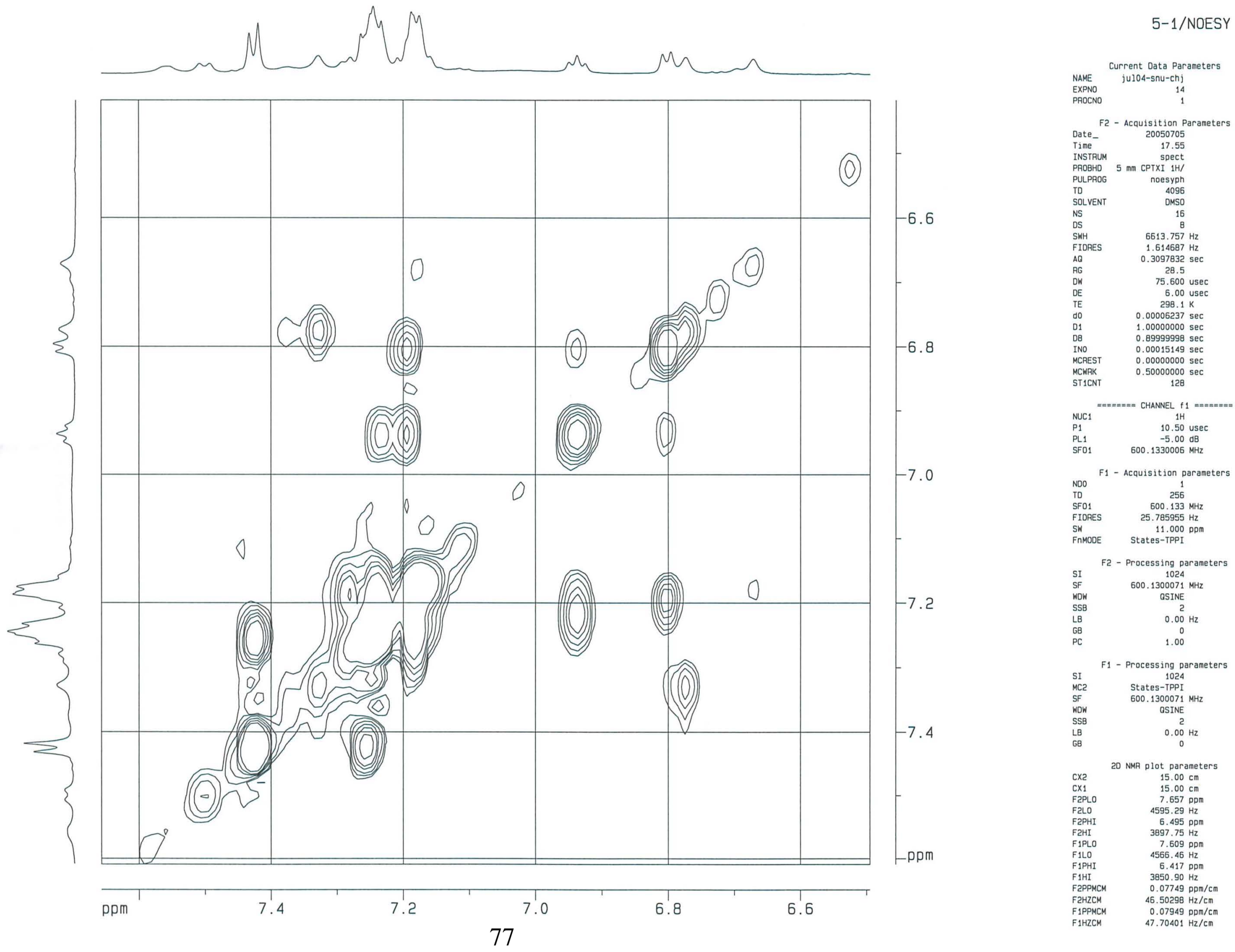


S71. HMBC (600 MHz) spectrum of halicylindramide H (3) in DMSO-d6

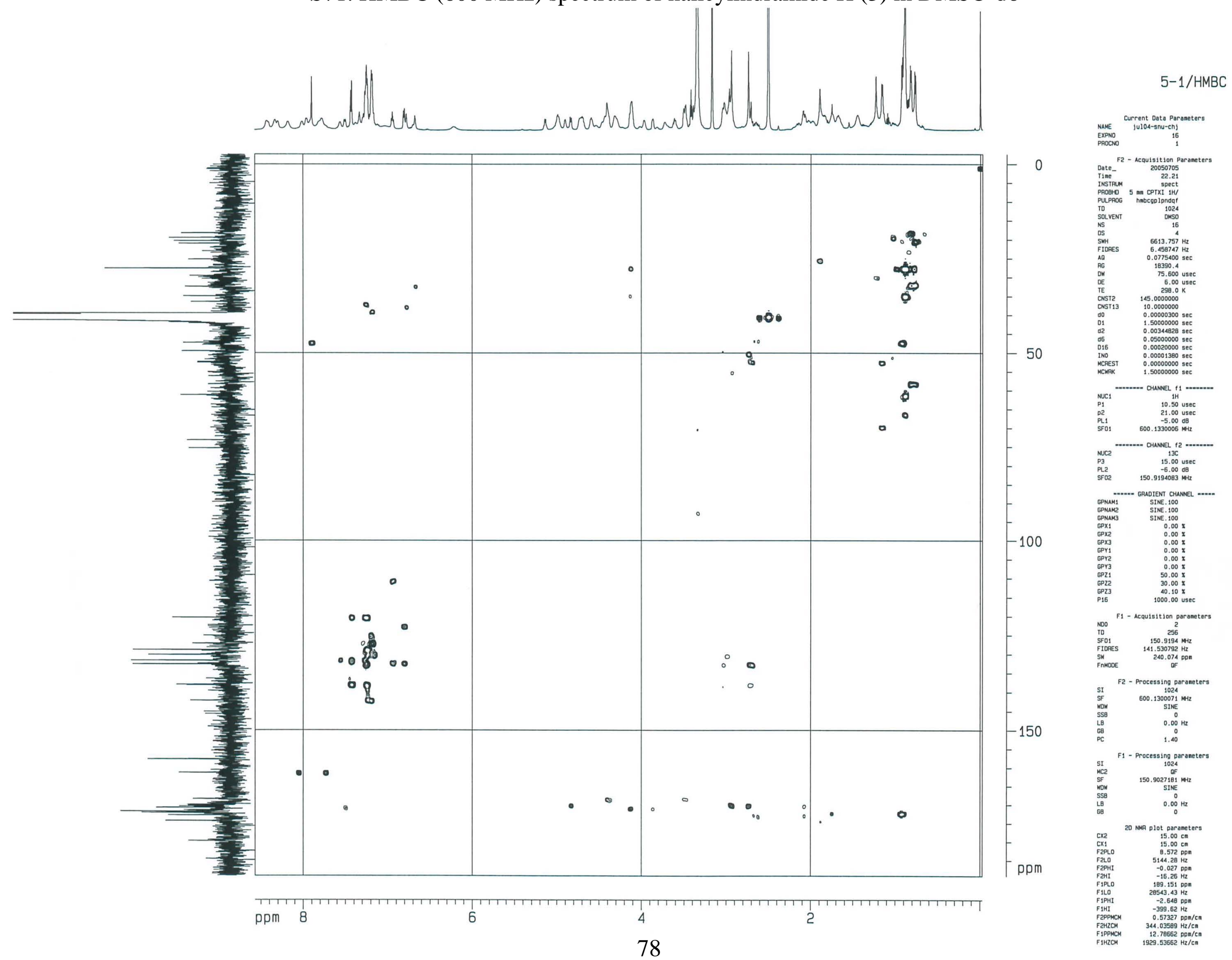


S72. Expanded HMBC (600 MHz) spectrum of halicylindramide H (3) in DMSO-d6

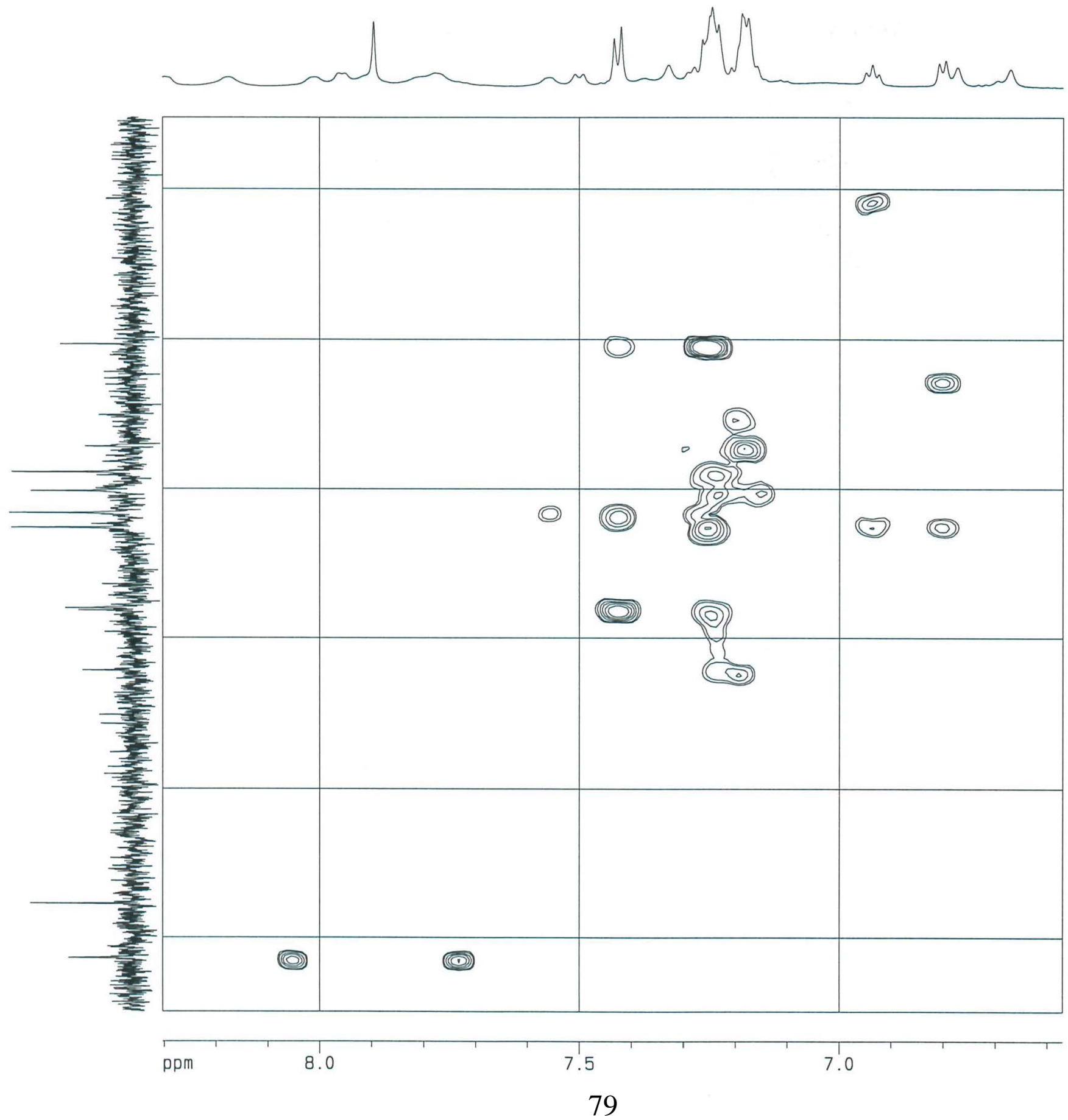

5-1/HMBC

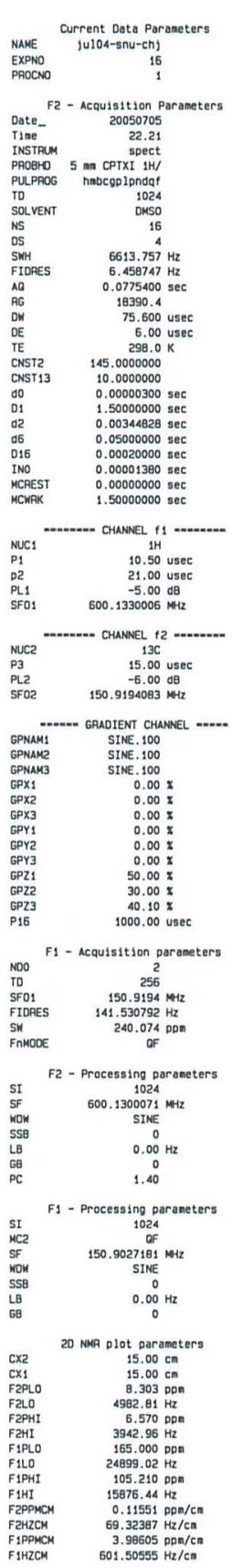


S73. Expanded HMBC (600 MHz) spectrum of halicylindramide H (3) in DMSO-d6

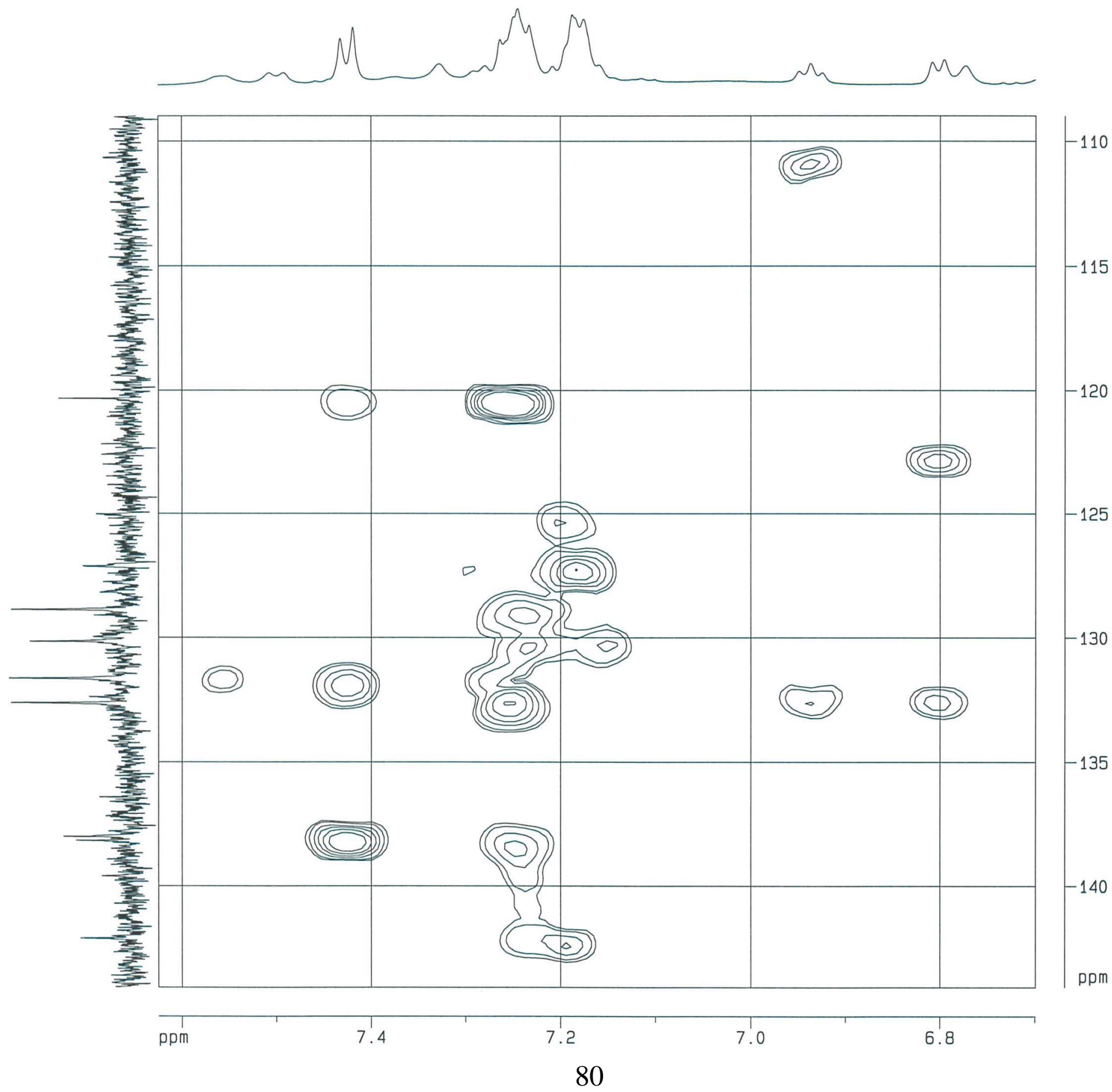

5-1/HMBC

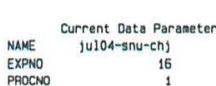

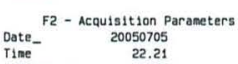

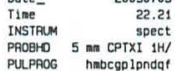

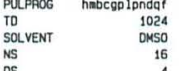

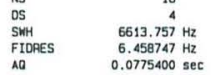

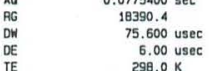

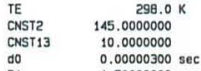

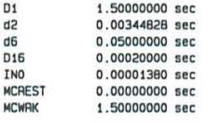

mect.

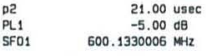

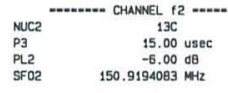

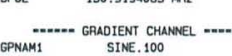

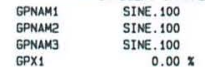

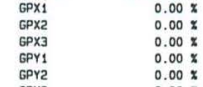

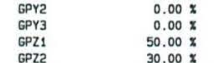

6223
P16

NoO
TD
TD - Acquisit tion paraneters
256

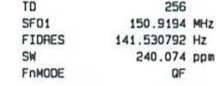

F2 - Processsing perraneters

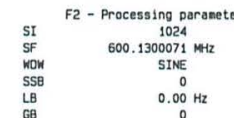

PC $\quad \begin{gathered}1.40 \\ \text { ST F1 - Processing paraneter } \\ \text { 1024 }\end{gathered}$

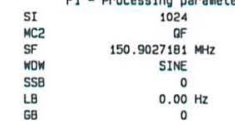

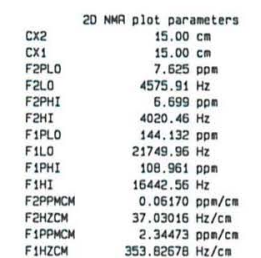


S74. Expanded HMBC (600 MHz) spectrum of halicylindramide H (3) in DMSO-d6

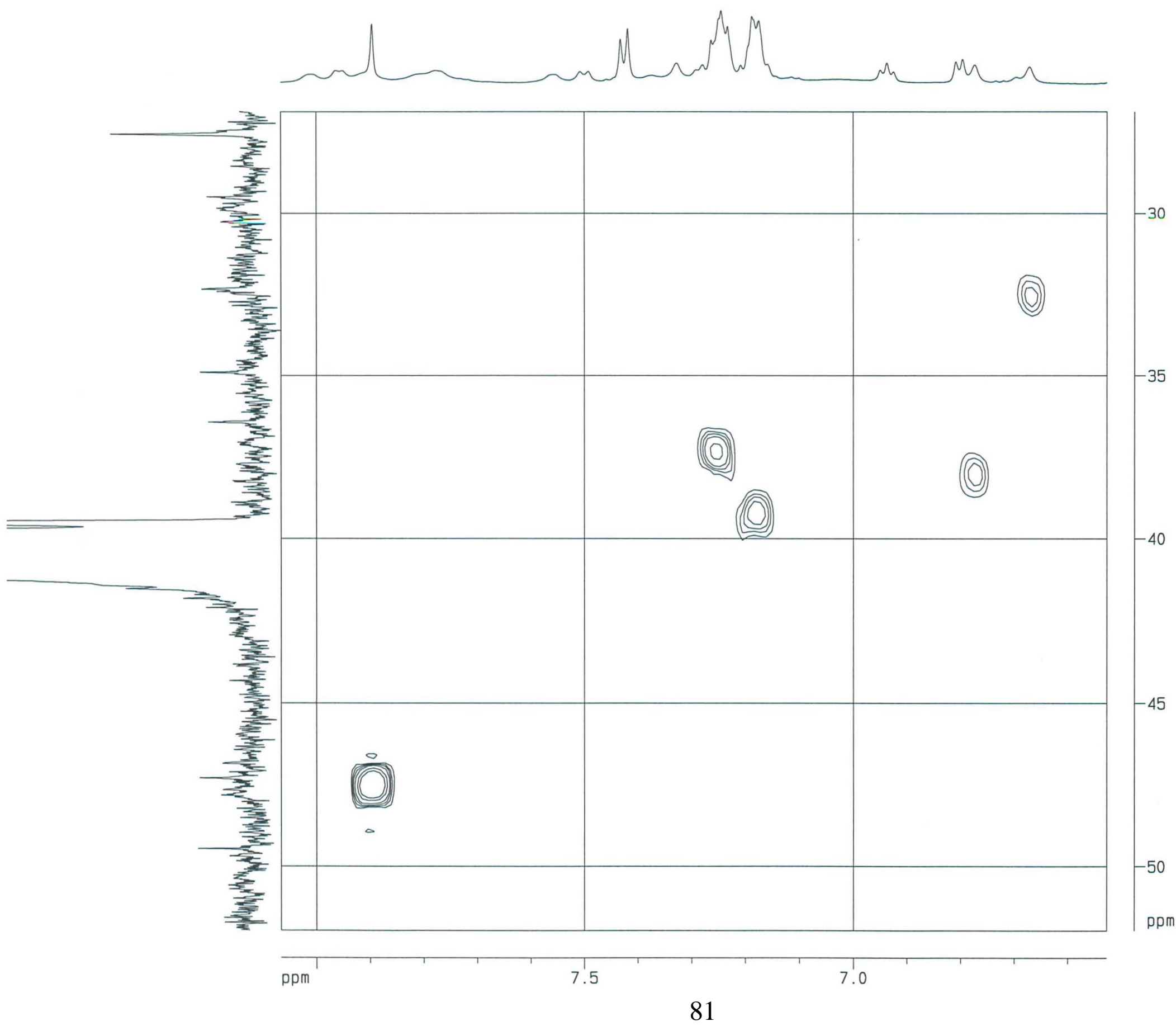

$5-1 /$ HMBC

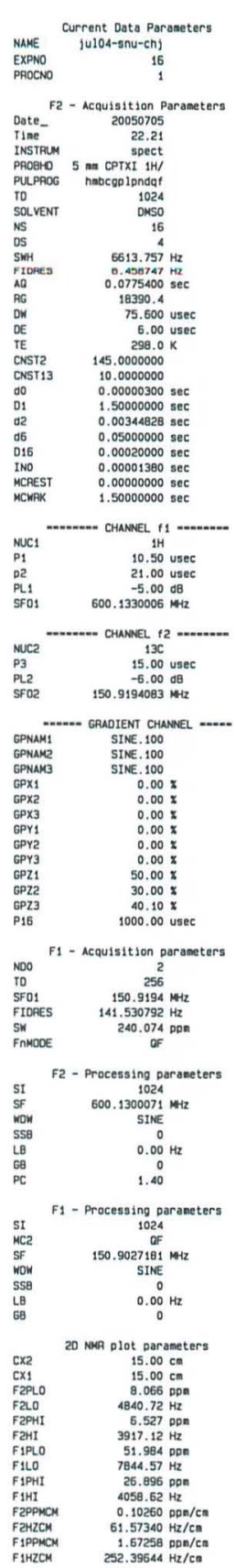


S75. Expanded HMBC (600 MHz) spectrum of halicylindramide H (3) in DMSO-d6

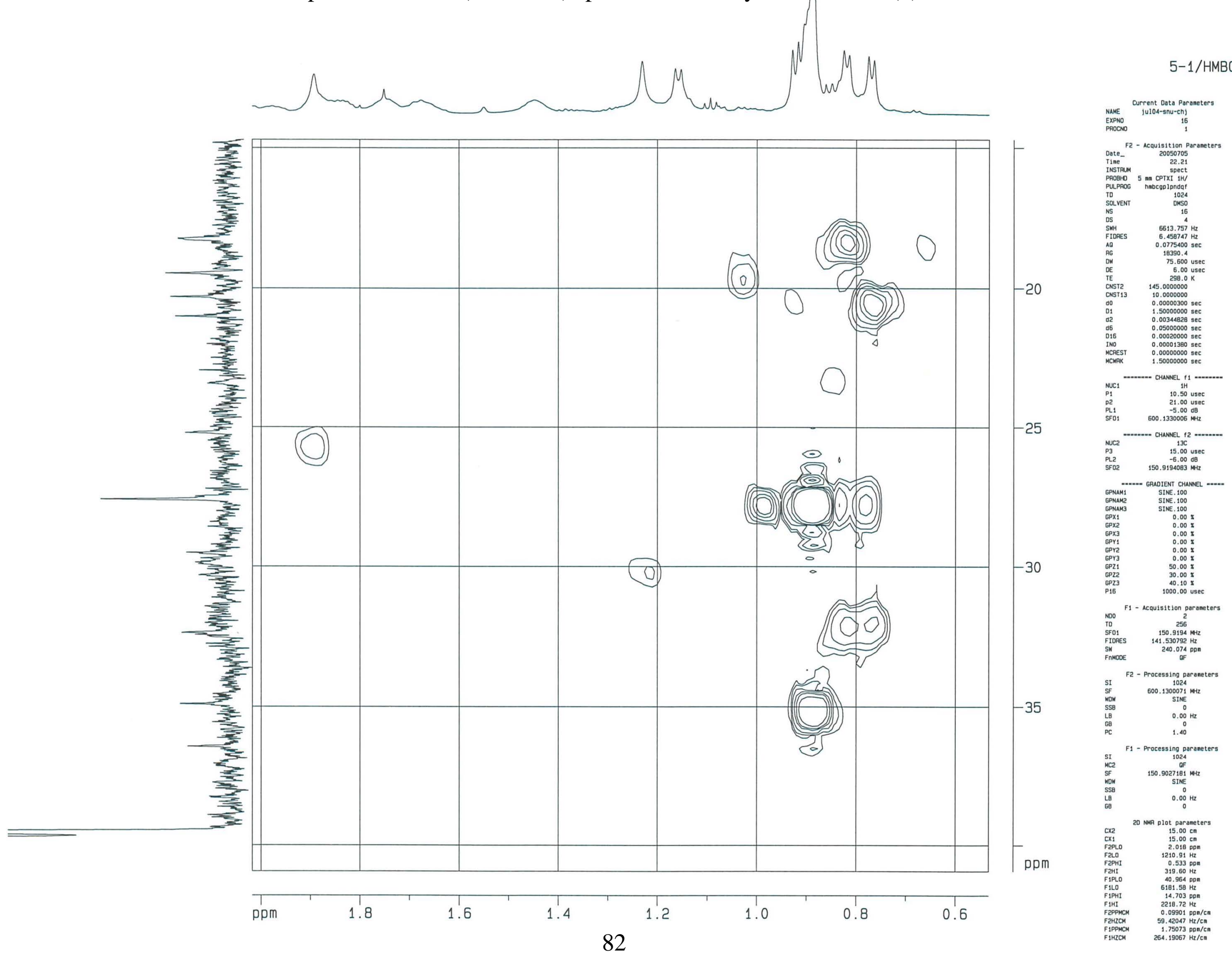


S76. Expanded HMBC (600 MHz) spectrum of halicylindramide H (3) in DMSO-d6

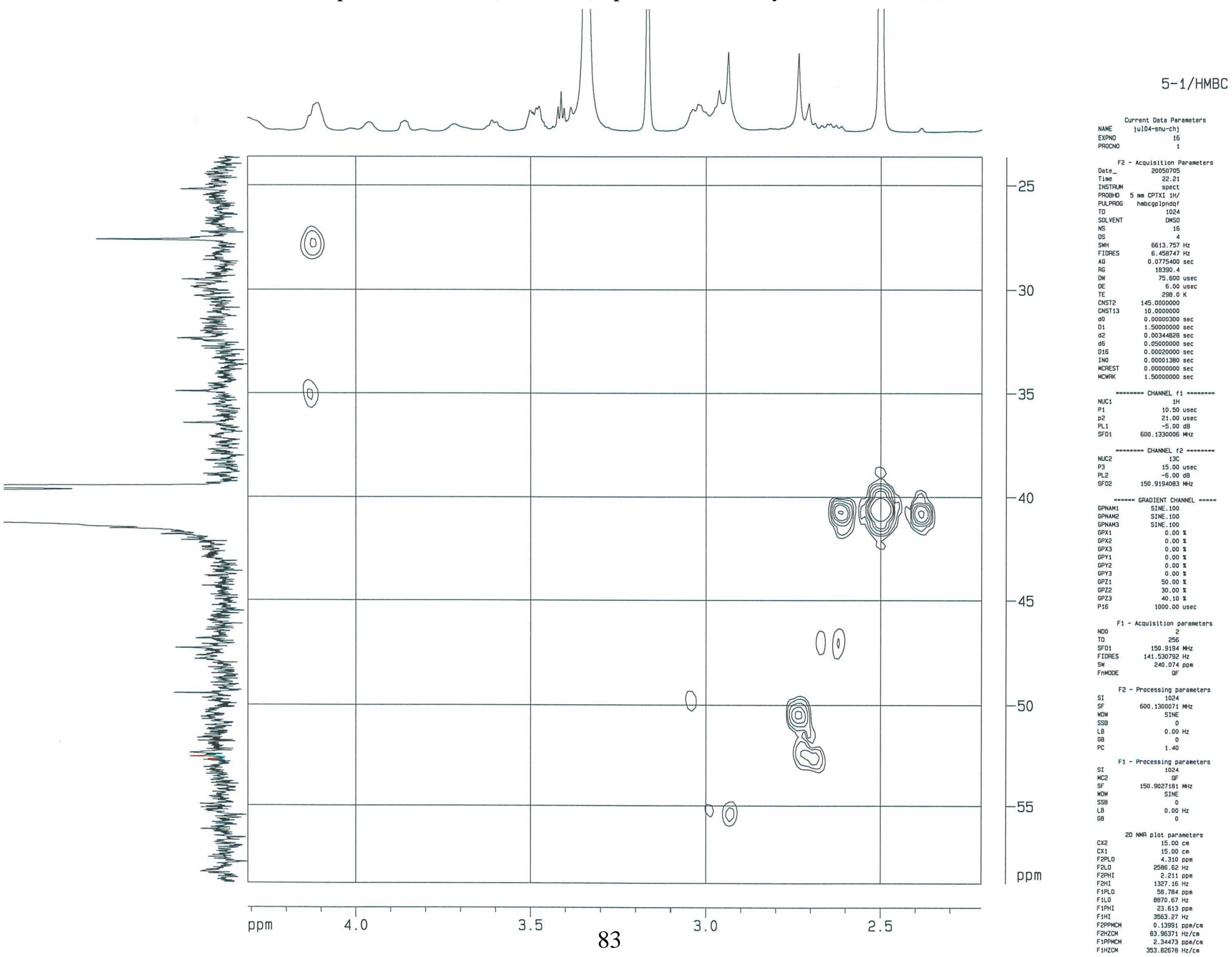


S77. Expanded HMBC (600 MHz) spectrum of halicylindramide H (3) in DMSO-d6

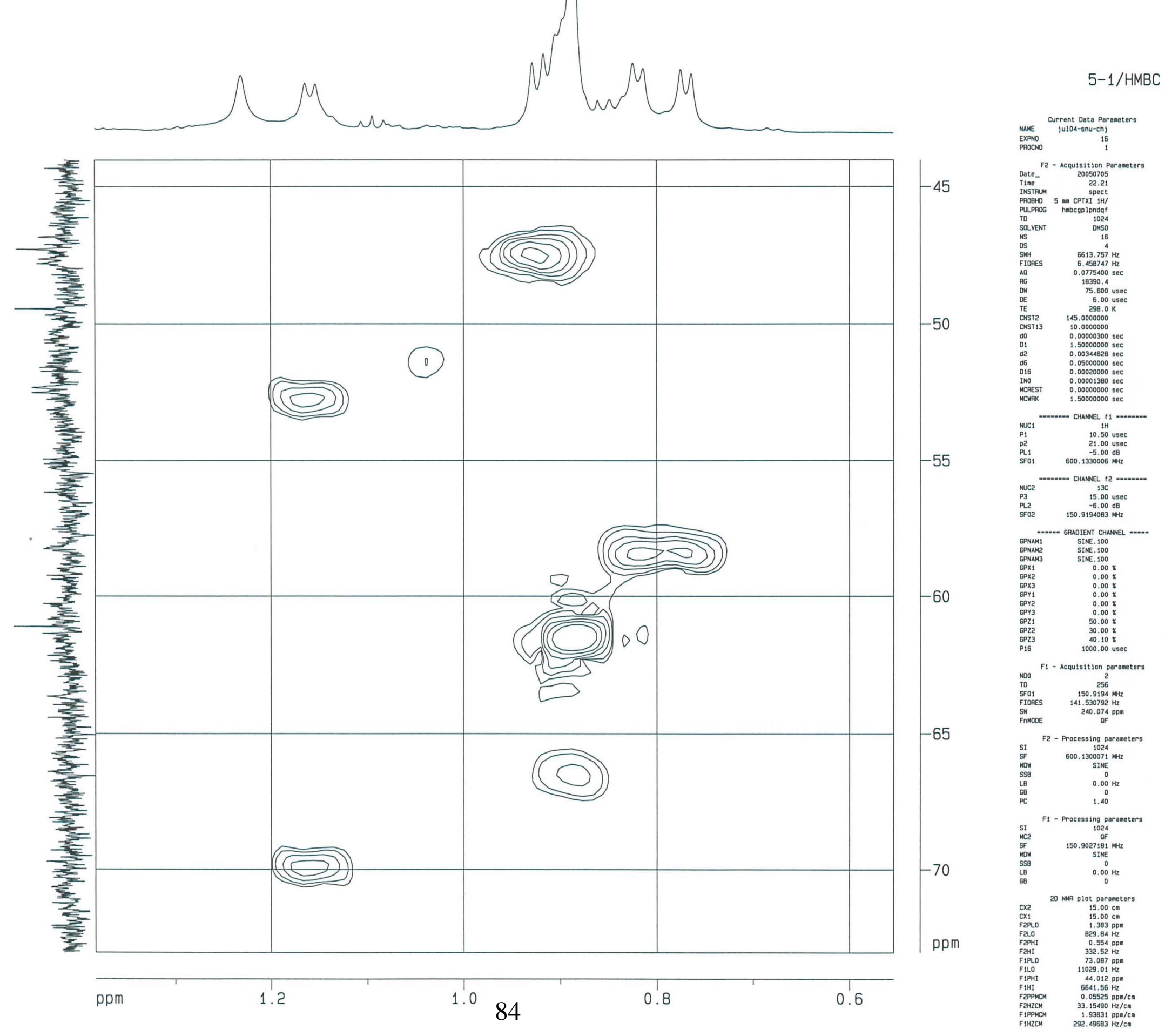


S78. Expanded HMBC (600 MHz) spectrum of halicylindramide H (3) in DMSO-d6

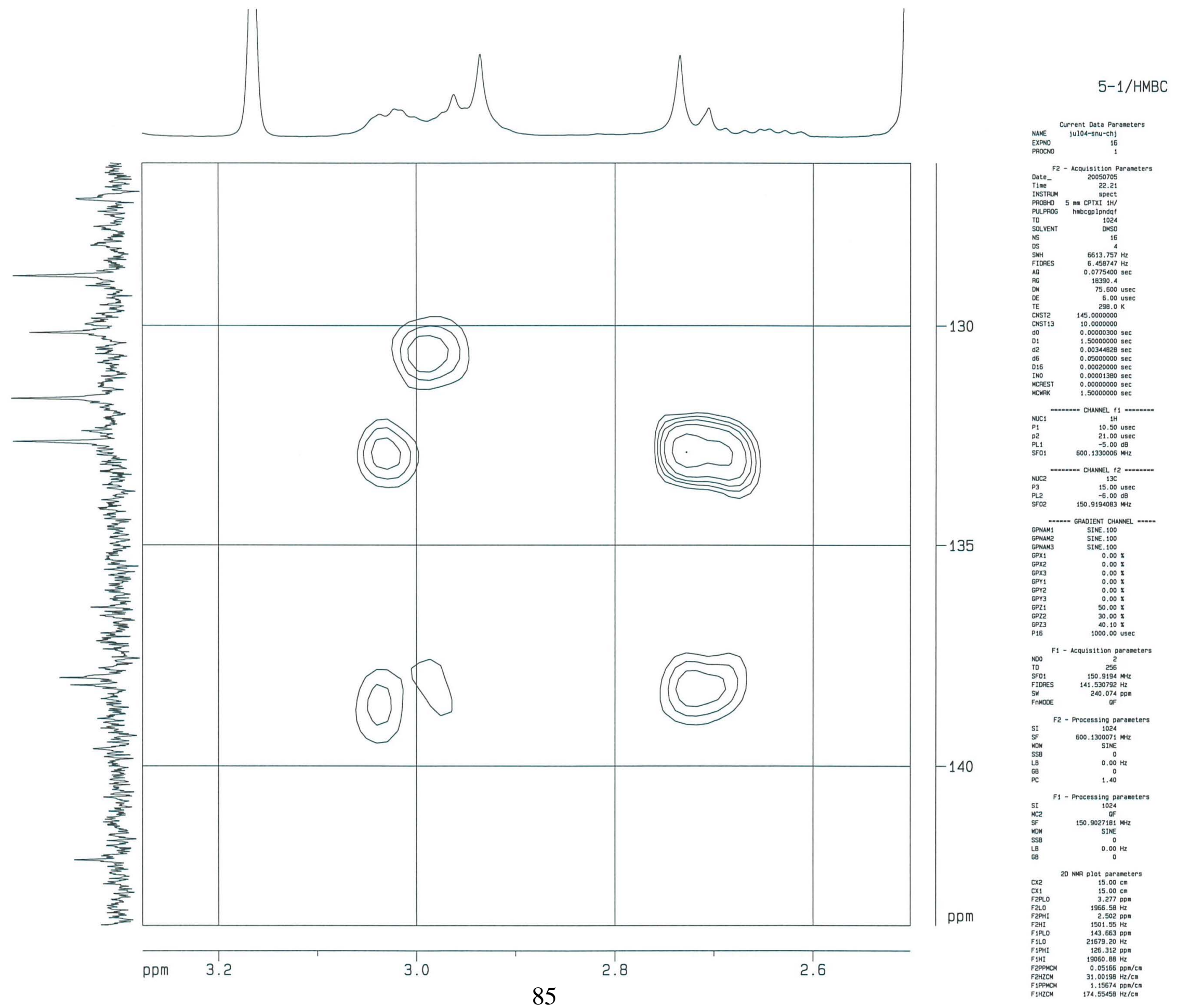


S79. Expanded HMBC (600 MHz) spectrum of halicylindramide H (3) in DMSO-d6

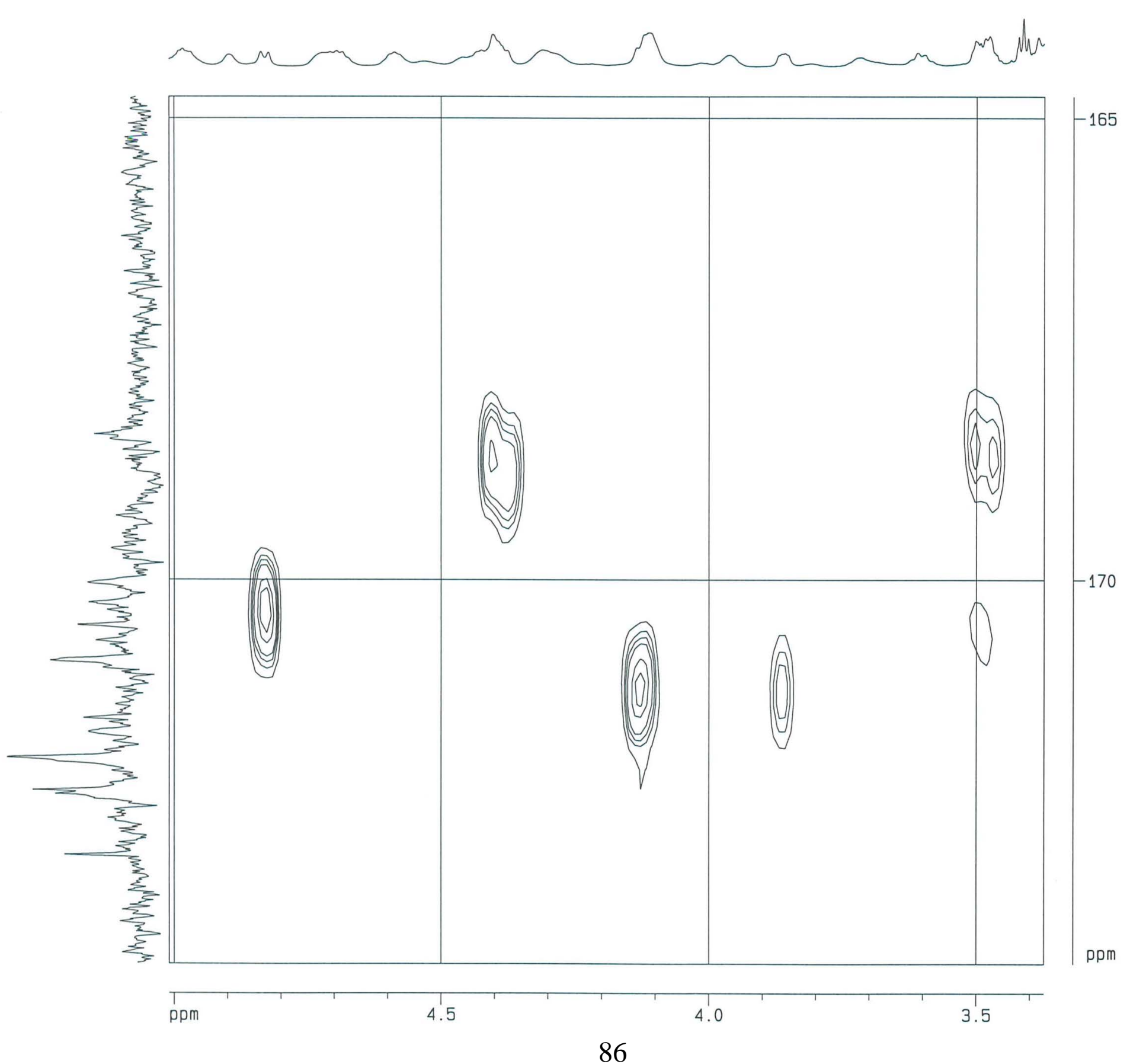

$5-1 /$ HMBC

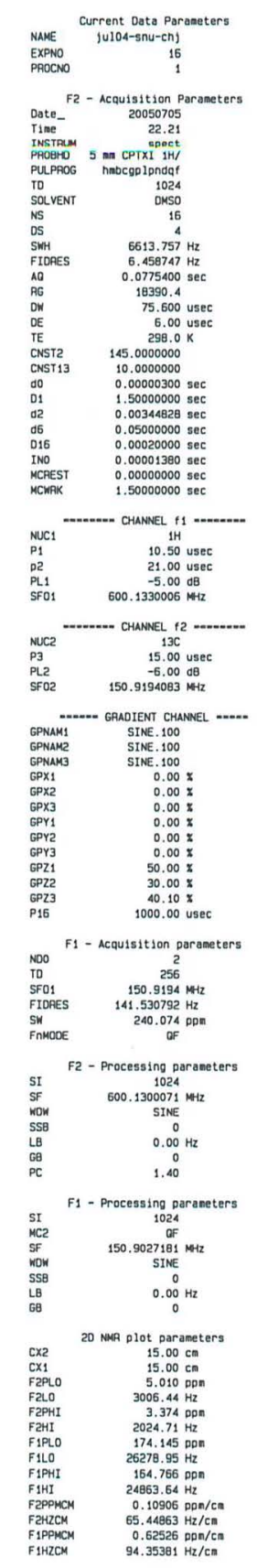


S80. Expanded HMBC (600 MHz) spectrum of halicylindramide H (3) in DMSO-d6
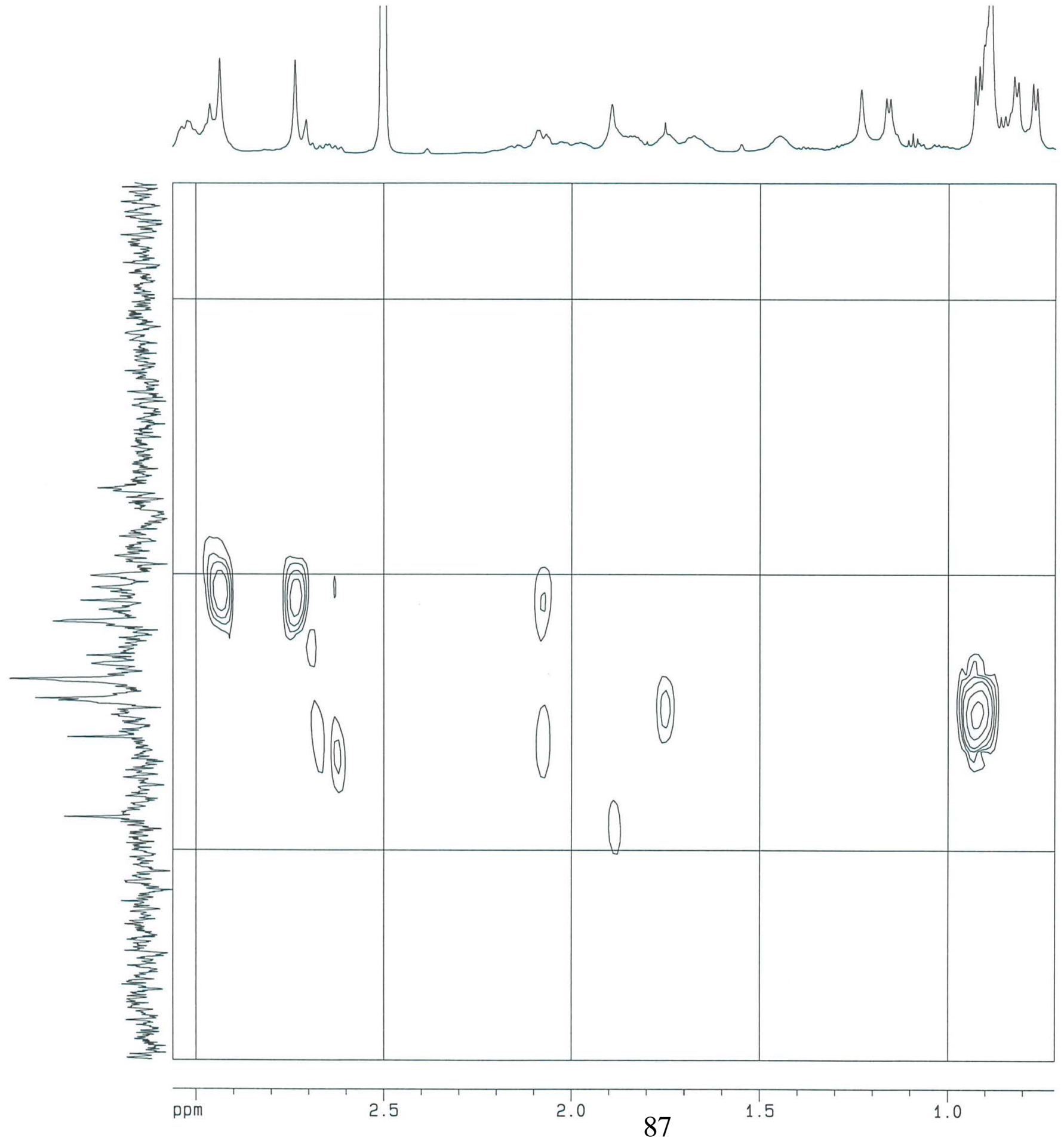

5-1/HMBC
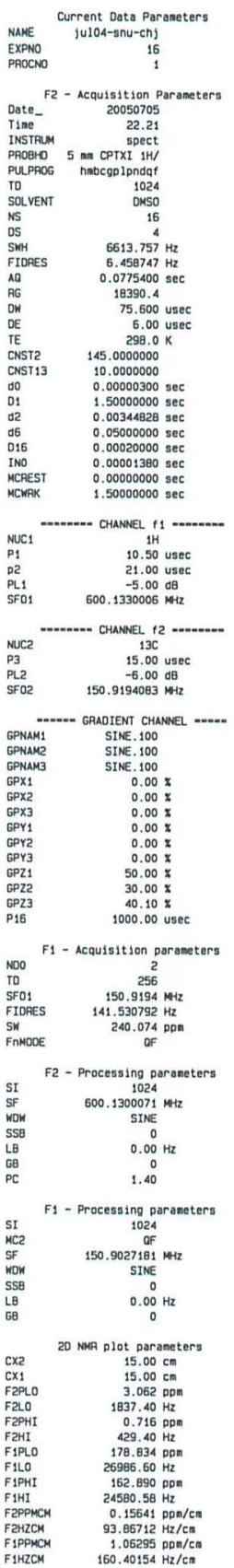
S81. Expanded HMBC (600 MHz) spectrum of halicylindramide $\mathrm{H}_{\text {(3) }}$ in DMSO-d6

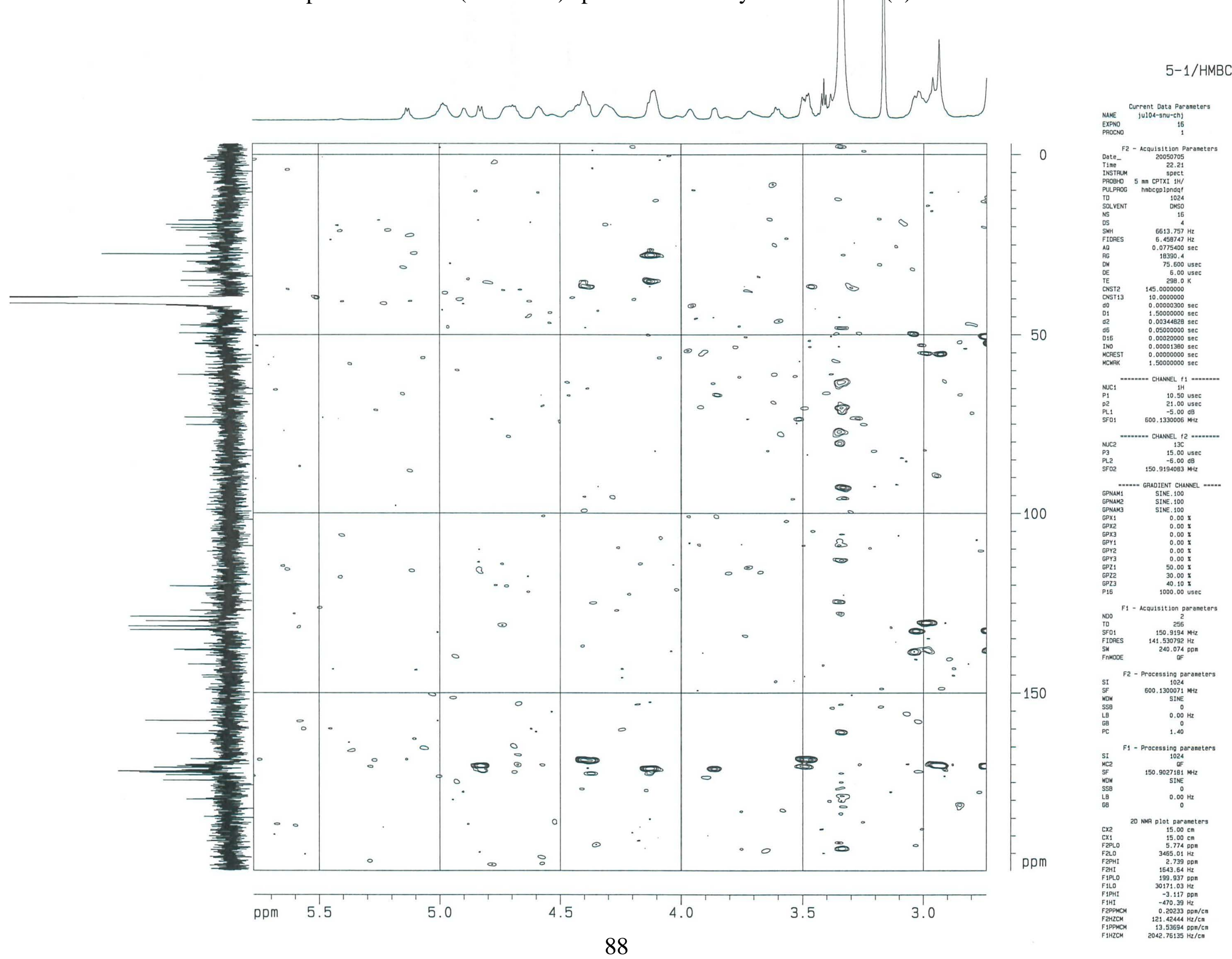


S82. Expanded HMBC (600 MHz) spectrum of halicylindramide H (3) in DMSO-d6
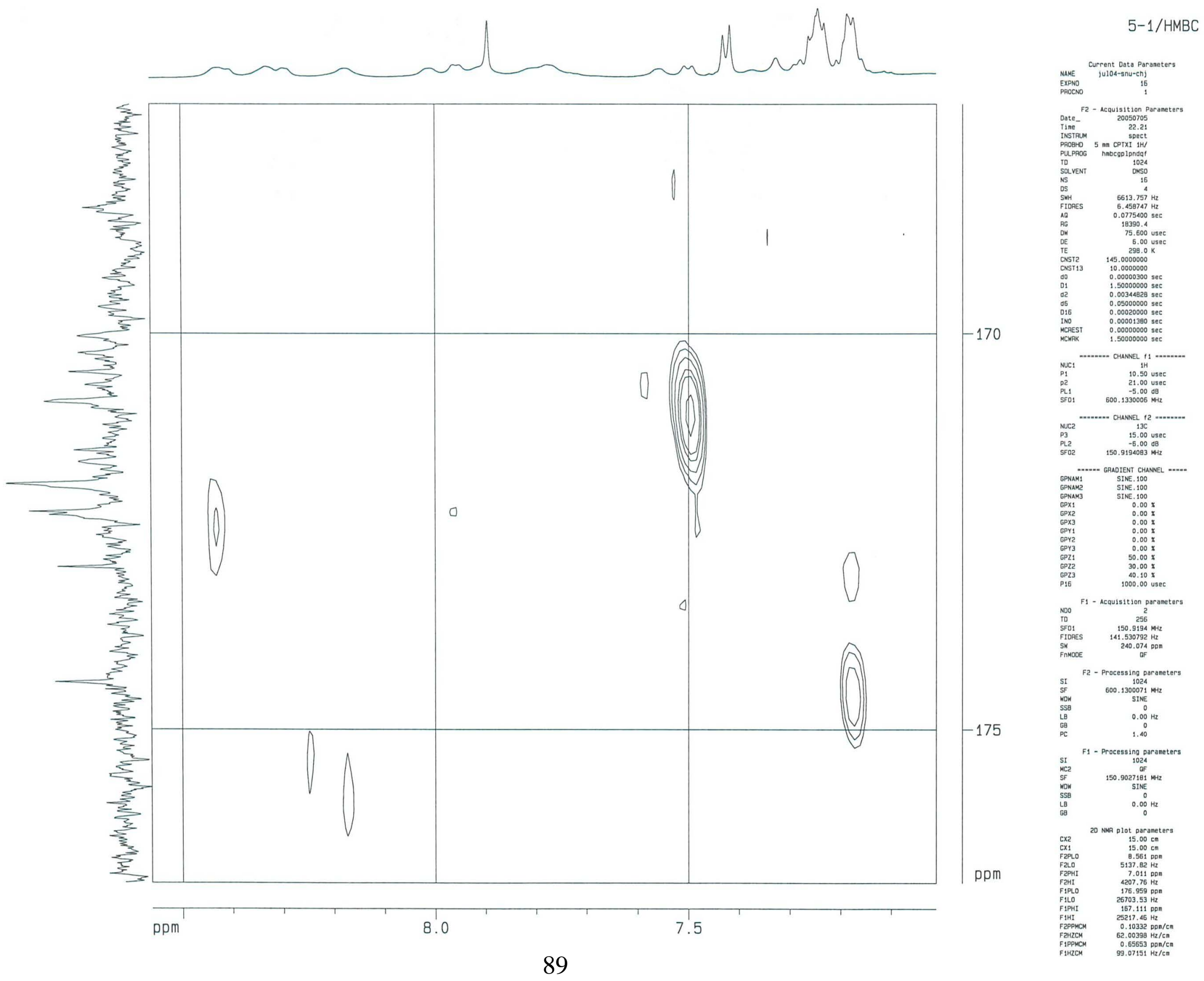
S83. Indirect binding of $\mathbf{1}, \mathbf{4}$ and $\mathbf{5}$ to the LBD of hFXR on BIAcore experiments

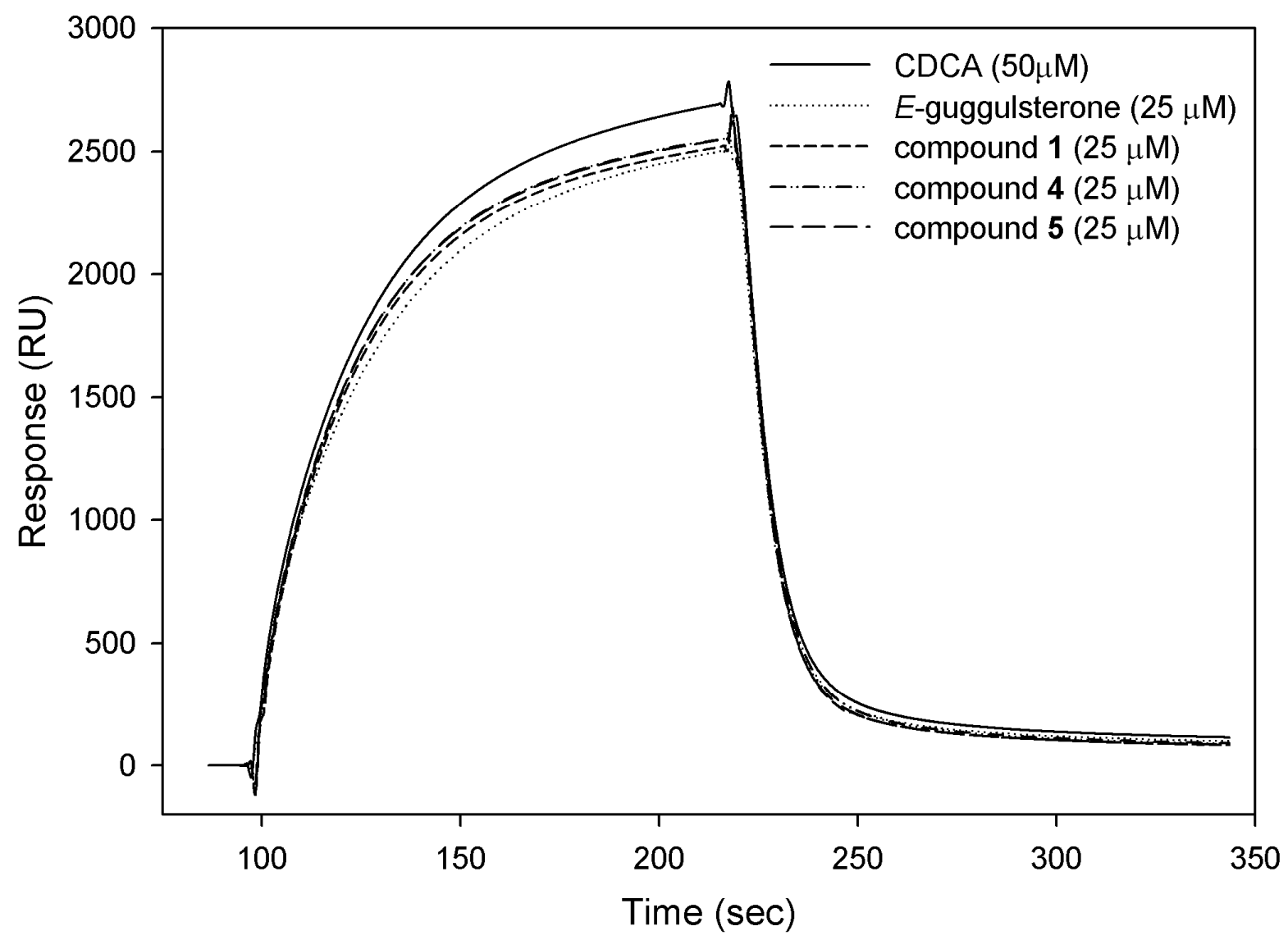


S84. Photograph of the animal specimen and taxonomic description
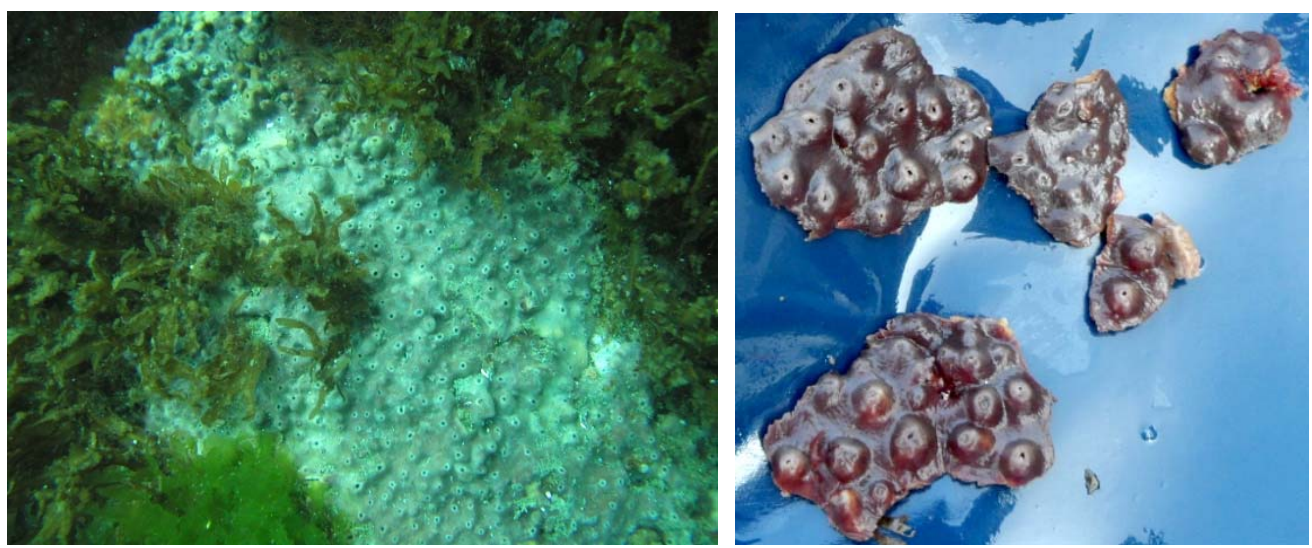

Porifera Demospongiae Haplosclerida Petrosina Petrosiidae

ID: Petrosia (Petrosia) 4921

Growth Form: Encrusting or lobate plate 2-5 mm thick

Colour: underwater pinky/brown grey with white rings around the oscules. Maroon on deck. Tints ethanol green

Oscules: on top of short conical fistules, $2 \mathrm{~mm}$ diameter.

Texture: smooth, compressible and brittle

Surface Features Fine microscoipic honeycomp pattern of ostia

Ectosome: Tangential reticulation of oxeas forming the surface mesh, with rarely protruding oxeas

Choanosome: unispicular Petrosid isotropic reticulation forming variable circular meshes, smaller at the surface and increasing in size deeper within the choanosome. No fibres. Abundant collagen pigmented red. Occassional paucispicular tracts leading to the ectosome.

Megascleres: One dominat size oxea, slightly cuved with sharp tips. (216-254 x 4.8-7.5 $\mu \mathrm{m})$, plus rarey thin oxeas $196 \times 1.63 \mu \mathrm{m}$.

Microscleres; Nil
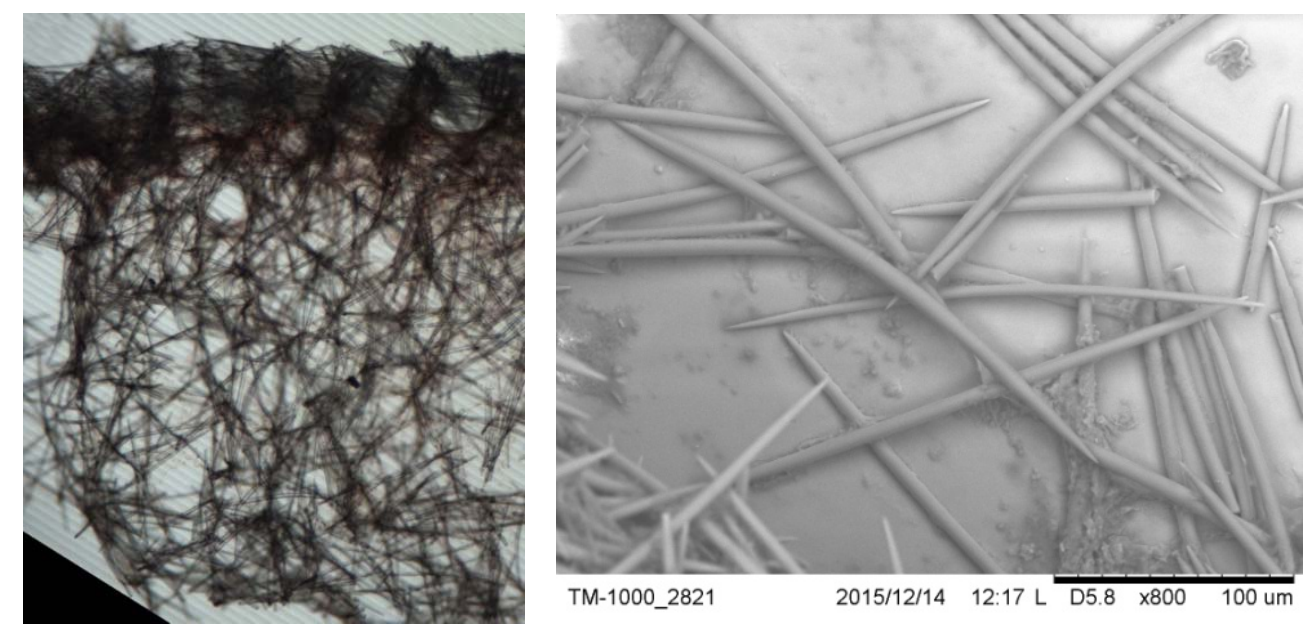
S85. Specific rotation values, ${ }^{1} \mathrm{H}$ NMR $(600 \mathrm{MHz})$ data and LR-ESI-MS data of four synthetic Dioias

D-Dioia-1: $[\alpha]_{\mathrm{D}}^{25}+20\left(c \mathrm{c} .1, \mathrm{H}_{2} \mathrm{O}\right) ; \mathrm{ECD}\left(0.5 \mathrm{mg} / \mathrm{mL}, \mathrm{CH}_{3} \mathrm{CN}\right), \lambda \max (\Delta \varepsilon) 318(-0.24), 265$ (21.49), and $239(-50.77) \mathrm{nm} ;{ }^{1} \mathrm{H}$ NMR $\delta_{\mathrm{H}}\left(\mathrm{DMSO}-d_{6}\right): 10.31(1 \mathrm{H}, \mathrm{brs}), 7.75$ (2H, brs), 7.24 $(1 \mathrm{H}, \mathrm{d}, J=7.6 \mathrm{~Hz}), 7.21(1 \mathrm{H}, \mathrm{t}, J=7.7 \mathrm{~Hz}), 6.97(1 \mathrm{H}, \mathrm{t}, J=7.3 \mathrm{~Hz}), 6.82(1 \mathrm{H}, \mathrm{d}, J=7.6 \mathrm{~Hz})$, $4.26(1 \mathrm{H}, \mathrm{dd}, J=9.5 \mathrm{~Hz}, 2.1 \mathrm{~Hz}), 3.33(1 \mathrm{H}, \mathrm{brs}) 2.31(1 \mathrm{H}, \mathrm{dd}, J=14.5 \mathrm{~Hz}, 9.7 \mathrm{~Hz}), 1.67(1 \mathrm{H}$, dd, $J=14.5 \mathrm{~Hz}, 2.4 \mathrm{~Hz})$, LR-ESI-MS m/z [M+H] 237 .

D-Dioia-2: $[\alpha]_{\mathrm{D}^{25}}-39\left(\right.$ c $\left.0.1, \mathrm{H}_{2} \mathrm{O}\right)$; $\mathrm{ECD}\left(0.5 \mathrm{mg} / \mathrm{mL}, \mathrm{CH}_{3} \mathrm{CN}\right), \lambda \max (\Delta \varepsilon) 315(-0.42), 263$ (20.07), and 240 (52.64) nm; ${ }^{1} \mathrm{H}$ NMR $\delta_{\mathrm{H}}\left(\mathrm{DMSO}-d_{6}\right): 10.31(1 \mathrm{H}, \mathrm{brs}), 7.91(2 \mathrm{H}, \mathrm{brs}), 7.37$ $(1 \mathrm{H}, \mathrm{d}, J=7.1 \mathrm{~Hz}), 7.22(1 \mathrm{H}, \mathrm{t}, J=7.7 \mathrm{~Hz}), 6.99(1 \mathrm{H}, \mathrm{t}, J=7.4 \mathrm{~Hz}), 6.84(1 \mathrm{H}, \mathrm{d}, J=7.6 \mathrm{~Hz})$, $3.81(1 \mathrm{H}, \mathrm{dd}, J=9.4 \mathrm{~Hz}, 3.9 \mathrm{~Hz}), 2.23(1 \mathrm{H}, \mathrm{dd}, J=14.3 \mathrm{~Hz}, 9.5 \mathrm{~Hz}), 1.76(1 \mathrm{H}, \mathrm{dd}, J=14.3$ Hz, $3.8 \mathrm{~Hz})$, LR-ESI-MS m/z [M+H] 237.

L-Dioia-1: $[\alpha]_{\mathrm{D}}^{25}-20\left(c 0.1, \mathrm{H}_{2} \mathrm{O}\right) ; \mathrm{ECD}\left(0.5 \mathrm{mg} / \mathrm{mL}, \mathrm{CH}_{3} \mathrm{CN}\right), \lambda \max (\Delta \varepsilon) 318(0.57), 266$ (-20.53), and $238(51.54) \mathrm{nm} ;{ }^{1} \mathrm{H}$ NMR $\delta_{\mathrm{H}}\left(\mathrm{DMSO}-d_{6}\right): 10.31(1 \mathrm{H}$, brs $), 7.75$ (2H, brs), 7.24 $(1 \mathrm{H}, \mathrm{d}, J=7.6 \mathrm{~Hz}), 7.21(1 \mathrm{H}, \mathrm{t}, J=7.7 \mathrm{~Hz}), 6.97(1 \mathrm{H}, \mathrm{t}, J=7.3 \mathrm{~Hz}), 6.82(1 \mathrm{H}, \mathrm{d}, J=7.6 \mathrm{~Hz})$ $4.26(1 \mathrm{H}, \mathrm{dd}, J=9.5 \mathrm{~Hz}, 2.1 \mathrm{~Hz}), 3.33(1 \mathrm{H}, \mathrm{brs}) 2.31(1 \mathrm{H}, \mathrm{dd}, J=14.5 \mathrm{~Hz}, 9.7 \mathrm{~Hz}), 1.67(1 \mathrm{H}$, dd, $J=14.5 \mathrm{~Hz}, 2.4 \mathrm{~Hz})$, LR-ESI-MS m/z [M+H $]^{+} 237$.

L-Dioia-2: $[\alpha]_{\mathrm{D}}^{25}+39\left(c 0.1, \mathrm{H}_{2} \mathrm{O}\right) ; \mathrm{ECD}\left(0.5 \mathrm{mg} / \mathrm{mL}, \mathrm{CH}_{3} \mathrm{CN}\right), \lambda \max (\Delta \varepsilon) 315(-0.42), 263$ (20.07), and 240 (52.64) nm; ${ }^{1} \mathrm{H}$ NMR $\delta_{\mathrm{H}}\left(\mathrm{DMSO}-d_{6}\right): 10.31(1 \mathrm{H}, \mathrm{brs}), 7.91(2 \mathrm{H}, \mathrm{brs}), 7.37$ $(1 \mathrm{H}, \mathrm{d}, J=7.1 \mathrm{~Hz}), 7.22(1 \mathrm{H}, \mathrm{t}, J=7.7 \mathrm{~Hz}), 6.99(1 \mathrm{H}, \mathrm{t}, J=7.4 \mathrm{~Hz}), 6.84(1 \mathrm{H}, \mathrm{d}, J=7.6 \mathrm{~Hz})$ $3.81(1 \mathrm{H}, \mathrm{dd}, J=9.4 \mathrm{~Hz}, 3.9 \mathrm{~Hz}), 2.23(1 \mathrm{H}, \mathrm{dd}, J=14.3 \mathrm{~Hz}, 9.5 \mathrm{~Hz}), 1.76(1 \mathrm{H}, \mathrm{dd}, J=14.3$ Hz, 3.8 Hz), LR-ESI-MS m/z [M+H] 237. 